\title{
Introduction
}

There are three main problems for the Navier-Stokes equations: global existence, global regularity and uniqueness. Global existence of weak solutions for the Cauchy problem was proved by Leray in 1934 (see [ler]). Next in 1951 Hopf [hop] proved existence of global weak solutions in a bounded domain for the Dirichlet problem with nonslip boundary conditions. The other two problems: existence of global regular solutions and uniqueness are still open. For the last fifty years many mathematicians have been working to solve these problems (see Kiselev and Ladyzhenskaya [kil], Ladyzhenskaya [lad 1-3], Serrin [ser], Fujita and Kato [fuk 1], Masuda [mas], Komatsu [kom], Caffarelli, Kohn and Nirenberg [ckn], Sohr [soh], Wiegner [wie 1-5], Nečas [nen, nrs], Neustupa [nen, nep 1-2, neu, nnp, npo 1-2], Penel [nnp, nep 1-2] etc.).

We can distinguish the following research directions: conditional regularity, blow-ups, existence of global regular special solutions (lower-dimensional, under special geometries) solutions. By conditional regularity we mean that some restrictions are imposed either on velocity or on pressure which imply regularity of weak solutions. The most important problem is to impose the weakest restrictions.

In this work we present a proof of existence and uniqueness of global regular special solutions to the Navier-Stokes equations in a bounded axially symmetric domain with boundary slip conditions under some restrictions on the initial conditions, the external force and the shape of the domain considered. The main aim is to prove the existence of solutions without restrictions on the magnitudes of the initial velocity and the external force. Hence we generalize [zaj 5] where a similar result in a cylindrical domain and with the slip coefficient $\gamma$ equal to zero was proved.

We have to underline that the proofs in this paper and in [zaj 5] are essentially different. However, in both cases the main step is the energy estimate for the azimuthal component of vorticity $\chi\left(\chi=\alpha_{\varphi}, \alpha=\operatorname{rot} v\right)$ which employs an idea of Ladyzhenskaya [lad 1] who proved such an estimate in the axially symmetric case. To prove this estimate she needed $\left.\chi\right|_{S}=0$ as the boundary condition. In [zaj 5] we showed that $\left.\chi\right|_{S}=0$ follows from the slip boundary conditions with slip coefficient $\gamma$ equal to zero and the fact that the domain considered is a cylinder. In this paper we consider the case with nonvanishing slip coefficient $\gamma$ and a general axially symmetric domain. Therefore $\left.\chi\right|_{S}$ is proportional to $k-\gamma /(2 \nu)$, where $k$ is the curvature of the boundary in the plane passing through the axis of symmetry, and $\nu$ is the viscosity coefficient. This fact makes the proof of the energy type estimate for $\chi$ much more difficult. Moreover, it implies heavy restrictions on the boundary and smallness of $k-\gamma /(2 \nu)$ in the norm of $V_{4,-\delta / 2}^{3 / 2}\left(S^{T}\right), \delta \geq 1$ (see Section 2 for the definition of the norm and also Lemmas 4.1 and 6.2). 
In this paper, similarly to [zaj 5], the most important estimates follow from the evolutionary problems for vorticity because the velocity $v$ is calculated from the elliptic problem $\operatorname{rot} v=\alpha, \operatorname{div} v=0,\left.v \cdot \bar{n}\right|_{S}=0$. To obtain such estimates we need appropriate boundary conditions for vorticity, where by "appropriate" we mean that $\left.\alpha\right|_{S}$ depends at most on $\left.v\right|_{S}$, and $\left.\bar{n} \cdot \nabla \alpha\right|_{S}$ at most on $\left.v_{, x}\right|_{S}$. This kind of boundary conditions follow from the slip boundary conditions in [zaj 5] as well as in this paper. Compared with [zaj 5] the proof of this fact in this paper is much more complicated (see Lemma 3.2). Moreover, the appropriate boundary conditions for vorticity imply that problems for $\alpha_{r}$ and $\alpha_{z}$ $\left(\alpha_{r}=\alpha \cdot \bar{e}_{r}, \alpha_{z}=\alpha \cdot \bar{e}_{z}, \bar{e}_{r}=(\cos \varphi, \sin \varphi, 0), \bar{e}_{z}=(0,0,1)\right)$ are coupled in boundary conditions (see (1.7)), in contrast to [zaj 5], where they are decoupled. This implies that the proof of the energy estimate for components $\alpha_{r}, \alpha_{z}$ of vorticity (solutions to problem (1.7) (see Lemma 4.4)) is much more difficult than the corresponding one in [zaj 5].

Finally, we underline that all estimates in this paper are done in the $L_{2}$-approach.

The main results of this paper are formulated in Theorems 1.1 (local existence) and 1.3 (global existence). The proof of global existence is divided into two main parts. First, local existence of solutions is proved by the method of successive approximations. In Section 6 a uniform bound for the constructed sequence is found (see Lemmas 6.1, 6.2). We underline that the time of local existence is inversely proportional to the expression which depends on norms describing the distance between the solution considered and the axially symmetric solution (see Definition 1.1). It is shown in Section 7 that the constructed sequence converges (see Theorem 7.7). In Section 8 global existence is proved step by step by continuing the local solution in time. To prove this we need some decay in time of the external force, and the existence time of the local solution must be sufficiently large. These conditions imply that no norms of initial data necessary for the local existence should increase with time. This yields global existence, which is shown in a series of lemmas in Section 8.

Inequalities necessary for the proof of local existence in Sections 6,7 are derived in Sections 4 and 5 .

We underline that the solution whose existence is proved in this paper has a much more general form than any solution obtained by examining stability of an axially symmetric solution (for more details see Section 9.7).

The paper is divided into nine sections. In Section 1 we formulate the main problems examined in this paper and also present the main results. In Section 2 we introduce notation. In particular, we formulate imbedding theorems for weighted Sobolev spaces. Boundary conditions for velocity and vorticity, and energy estimates for weak solutions are found in Section 3. In Section 9 we present a review of results on global solutions to the Navier-Stokes equations, giving the state of the art in one of the seven millennium problems. Other sections are described above.

We use the abbreviation r.h.s. (1.h.s.) for right-hand side (left-hand side). Formulas and theorems are numbered by section, except those in Section 9 which are numbered by subsection.

Acknowledgments. The author thanks Prof. J. Zabczyk for important remarks which allowed improving the form of this paper. 


\section{Formulation of main results}

In this work we consider a motion of a viscous incompressible fluid in a bounded axially symmetric domain $\Omega \subset \mathbb{R}^{3}$ under the boundary slip conditions (see [lal]):

$$
\begin{aligned}
& v_{, t}+v \cdot \nabla v-\operatorname{div} \mathbb{T}(v, p)=f \quad \text { in } \Omega^{T}=\Omega \times(0, T), \\
& \operatorname{div} v=0 \quad \text { in } \Omega^{T} \text {, } \\
& v \cdot \bar{n}=0 \quad \text { on } S^{T}=S \times(0, T), \\
& \bar{n} \cdot \mathbb{T}(v, p) \cdot \bar{\tau}_{\alpha}+\gamma v \cdot \bar{\tau}_{\alpha}=0, \quad \alpha=1,2, \quad \text { on } S^{T}, \\
& \left.v\right|_{t=0}=v(0) \quad \text { in } \Omega,
\end{aligned}
$$

where $v=v(x, t)=\left(v_{1}(x, t), v_{2}(x, t), v_{3}(x, t)\right) \in \mathbb{R}^{3}$ is the velocity vector field, $p=$ $p(x, t) \in \mathbb{R}$ the pressure, $f=f(x, t)=\left(f_{1}(x, t), f_{2}(x, t), f_{3}(x, t)\right) \in \mathbb{R}^{3}$ the external force field, $\bar{n}$ is the unit outward vector normal to $S=\partial \Omega, \bar{\tau}_{1}, \bar{\tau}_{2}$ are the unit vectors tangent to $S, \gamma>0$ is the constant slip coefficient and $\mathbb{T}(v, p)$ is the stress tensor of the form

$$
\mathbb{T}(v, p)=\left\{\nu\left(v_{i, x_{j}}+v_{j, x_{i}}\right)-p \delta_{i j}\right\}_{i, j=1,2,3} \equiv \nu \mathbb{D}(v)-p I,
$$

where $\nu$ is the constant positive viscosity coefficient, $\mathbb{D}(v)$ is the dilatation tensor and $I$ is the unit matrix.

We should underline that another boundary condition for system $(1.1)_{1,2}$ is the nonslip condition

$$
\left.v\right|_{S}=0
$$

Letting $\gamma \rightarrow \infty$ in $(1.1)_{4}$ we obtain from (1.1) $)_{3,4}$ the nonslip boundary condition (1.3).

Moreover, $x=\left(x_{1}, x_{2}, x_{3}\right)$ are the Cartesian coordinates. We assume that $\Omega \subset \mathbb{R}^{3}$ is a bounded axially symmetric domain. The cylindrical coordinates $(r, \varphi, z)$ are introduced by the relation $x_{1}=r \cos \varphi, x_{2}=r \sin \varphi, x_{3}=z$, and the $z$ axis is the axis of symmetry of $\Omega . S$ is described by $\psi(r, z)=0, r \in[0, R], z \in[-a, a]$, which in a neighbourhood of $r=R$ can be expressed by $r=\psi_{1}(z)$ and in a neighbourhood of $|z|=a$ by $z=\psi_{2}(r)$.

Let $\bar{e}_{r}=(\cos \varphi, \sin \varphi, 0), \bar{e}_{\varphi}=(-\sin \varphi, \cos \varphi, 0), \bar{e}_{z}=(0,0,1)$ be the unit vectors along the lines $r, \varphi, z$, respectively. Let $k$ be any vector. Then $k_{r}=k \cdot \bar{e}_{r}, k_{\varphi}=k \cdot \bar{e}_{\varphi}$, $k_{z}=k \cdot \bar{e}_{z}$.

Let us introduce the quantities

$$
h=v_{r, \varphi} \bar{e}_{r}+v_{\varphi, \varphi} \bar{e}_{\varphi}+v_{z, \varphi} \bar{e}_{z}, \quad q=p_{, \varphi}, \quad w=v_{\varphi} .
$$

DeFinition 1.1. By an axially symmetric solution to (1.1) we mean a solution such that $h=0, q=0, w=0$. 
To show existence of such solutions we have to assume that $\left.h\right|_{t=0}=h(0)=0,\left.w\right|_{t=0}=$ $w(0)=0, f_{\varphi}=0, g=f_{r, \varphi} \bar{e}_{r}+f_{\varphi, \varphi} \bar{e}_{\varphi}+f_{z, \varphi} \bar{e}_{z}=0$.

1.1. Reformulation of the main problem. To prove the existence of solutions to problem (1.1) we replace it by a system of problems. From [zaj 5, Ch. 4] we have

$$
\begin{array}{ll}
h_{, t}-\nu \operatorname{div} \mathbb{D}(h)+\nabla q=-v \cdot \nabla h-h \cdot \nabla v+g \equiv G & \text { in } \Omega^{T}, \\
\operatorname{div} h=0 & \text { in } \Omega^{T}, \\
h \cdot \bar{n}=0 & \text { on } S^{T}, \\
\nu \bar{n} \cdot \mathbb{D}(h) \cdot \bar{\tau}_{\alpha}+\gamma h \cdot \bar{\tau}_{\alpha}=0, \quad \alpha=1,2, & \text { on } S^{T}, \\
\left.h\right|_{t=0}=h(0) & \text { in } \Omega,
\end{array}
$$

where $g=f_{r, \varphi} \bar{e}_{r}+f_{\varphi, \varphi} \bar{e}_{\varphi}+f_{z, \varphi} \bar{e}_{z}, h(0)=v_{r, \varphi} \bar{e}_{r}+v_{\varphi, \varphi} \bar{e}_{\varphi}+\left.v_{z, \varphi} \bar{e}_{z}\right|_{t=0}$ and $v$ is treated as a given vector.

For given $v, q, h$ we have (see [zaj 5, Ch. 4] and (3.2))

$$
\begin{array}{ll}
w_{, t}+v \cdot \nabla w+\frac{v_{r}}{r} w-\nu \Delta w+\nu \frac{w}{r^{2}}=\frac{1}{r} q+\frac{2 \nu}{r^{2}} h_{r}+f_{\varphi} & \text { in } \Omega^{T}, \\
\nu \bar{n} \cdot \nabla w=-\gamma w+\nu \frac{a_{1}}{r} w & \text { on } S^{T}, \\
\left.w\right|_{t=0}=w(0) & \text { in } \Omega,
\end{array}
$$

where the boundary $S$ is described by the equation $\psi(r, z)=0$, so $a_{1}=\psi_{, r} / \sqrt{\psi_{, r}^{2}+\psi_{, z}^{2}}$, $a_{2}=\psi_{, z} / \sqrt{\psi_{, r}^{2}+\psi_{, z}^{2}}$.

Let us introduce the cylindrical components of vorticity (see [zaj 5, Ch. 4])

$$
\begin{aligned}
\alpha_{1} & =\alpha_{r}=\frac{1}{r}\left(v_{z, \varphi}-r v_{\varphi, z}\right), \\
\alpha_{2}=\alpha_{\varphi} & =v_{r, z}-v_{z, r} \equiv \chi, \\
\alpha_{3}=\alpha_{2} & =\frac{1}{r}\left[\left(r v_{\varphi}\right)_{, r}-v_{r, \varphi}\right] .
\end{aligned}
$$

Applying the rot operator to (1.1) ${ }_{1}$ and using (3.6)-(3.8) (see Lemma 3.2) we obtain

$$
\begin{array}{ll}
\alpha_{1, t}+v \cdot \nabla \alpha_{1}-\alpha_{1} v_{r, r}-\frac{\alpha_{2}}{r} h_{r}-\alpha_{3} v_{r, z}+\frac{2 \nu}{r^{2}}\left(h_{r, z}-h_{z, r}\right) & \\
\quad+\frac{\nu \alpha_{1}}{r^{2}}-\nu \Delta \alpha_{1}=F_{1} & \text { in } \Omega^{T}, \\
\alpha_{3, t}+v \cdot \nabla \alpha_{3}-\left(\alpha_{1} v_{z, r}+\alpha_{3} v_{z, z}\right)-\frac{\alpha_{2}}{r} h_{z}-\nu \Delta \alpha_{3}=F_{3} & \text { in } \Omega^{T}, \\
\bar{\tau}_{2} \cdot \alpha=-\frac{2 a_{1}}{r} w+\frac{\gamma}{\nu} w \equiv g_{1} & \text { on } S^{T}, \\
(\bar{n} \cdot \alpha)_{, n}=\beta_{1} h_{r}+\beta_{2} h_{z}+\beta_{3} w_{, r}+\beta_{4} w_{, z}+\beta_{5} w \equiv g_{2} & \text { on } S^{T}, \\
\left.\alpha_{1}\right|_{t=0}=\alpha_{1}(0),\left.\alpha_{3}\right|_{t=0}=\alpha_{2}(0) & \text { in } \Omega,
\end{array}
$$

where $\beta_{i}, i=1, \ldots, 5$, depend on $a_{1}, a_{2}$ and their derivatives with respect to $r$ and $z$ (see Lemma 3.2). Moreover, $F=\operatorname{rot} f, F_{1}=F \cdot \bar{e}_{r}, F_{3}=F \cdot \bar{e}_{z}$. 
We shall denote a solution of (1.7) by $\alpha^{\prime}=\left(\alpha_{1}, \alpha_{3}\right)$. Finally, $\alpha_{2}$ is a solution to the problem

$$
\begin{array}{lr}
\alpha_{2, t}+v \cdot \nabla \alpha_{2}+\frac{w}{r} \alpha_{1}-\alpha \cdot \nabla w-\frac{\alpha_{2}}{r} v_{r}-\frac{2 \nu}{r^{2}}\left(\frac{1}{r} h_{z, \varphi}-h_{\varphi, z}\right) & \\
\quad+\frac{\nu \alpha_{2}}{r^{2}}-\nu \Delta \alpha_{2}=F_{2} & \text { in } \Omega^{T}, \\
\alpha_{2}=2(k-\gamma /(2 \nu)) v \cdot \bar{\tau}_{2} & \text { on } S^{T}, \\
\left.\alpha_{2}\right|_{t=0}=\alpha_{2}(0) \equiv \chi(0), &
\end{array}
$$

where $\bar{\tau}_{2}=a_{2} \bar{e}_{r}-a_{1} \bar{e}_{z}, \bar{n}=a_{1} \bar{e}_{r}+a_{2} \bar{e}_{z}, \bar{\tau}_{2} \cdot \alpha=a_{2} \alpha_{1}-a_{1} \alpha_{3}, \bar{n} \cdot \alpha=a_{1} \alpha_{1}+a_{2} \alpha_{3}$, $\partial_{n}=\bar{n} \cdot \nabla, k$ is the curvature of a curve $S^{\prime}$ which generates $S$ by rotating around the $z$ axis and $F_{2}=F \cdot \bar{e}_{\varphi}$.

Finally, $v$ and $p$ are calculated from the elliptic problems

$$
\begin{array}{ll}
\operatorname{rot} v=\alpha & \text { in } \Omega, \\
\operatorname{div} v=0 & \text { in } \Omega, \\
v \cdot \bar{n}=0 & \text { on } S,
\end{array}
$$

and

$$
\begin{array}{ll}
\Delta p=-\nabla v \cdot \nabla v+\operatorname{div} f & \text { in } \Omega, \\
\frac{\partial p}{\partial n}=f \cdot \bar{n}+\nu \bar{n} \cdot \Delta v-\bar{n} \cdot v \cdot \nabla v & \text { on } S .
\end{array}
$$

In [zaj 5, Ch. 3] we showed that (1.10) is necessary and sufficient for equivalence of problems (1.1) and (1.7), (1.8), (1.9). However to prove global existence with large data it is not enough to examine problems (1.7)-(1.9) only. To obtain necessary estimates we also need problems (1.4), (1.5).

Now we express boundary conditions $(1.10)_{2}$ in the form depending at most on the first derivatives of velocity. For this we assume that in a neighbourhood of $S$ there is defined an orthonormal system of vectors $\bar{n}, \bar{\tau}_{1}, \bar{\tau}_{2}$. With these vectors curvilinear coordinates $n, \tau_{1}, \tau_{2}$ such that $\nabla n \cdot \nabla \tau_{\alpha}=0,|\nabla n|=1, \nabla \tau_{\alpha} \cdot \nabla \tau_{\beta}=\delta_{\alpha \beta}, \alpha, \beta=1,2$, are connected. Then in the neighbourhood of $S$ we have

$$
\Delta v=v_{, n n}+v_{, \tau_{\alpha} \tau_{\alpha}}+v_{, n} \Delta n+v_{, \tau_{\alpha}} \Delta \tau_{\alpha},
$$

where the summation convention over the repeated indices $\alpha$ is assumed. Next

$$
\begin{gathered}
\bar{n} \cdot v_{, n n}=v_{n, n n}+v_{n} \bar{n} \cdot \bar{n}_{, n n}+2 v_{\tau_{\alpha, n}} \bar{n} \cdot \bar{\tau}_{\alpha, n}+v_{\tau_{\alpha}} \bar{n} \cdot \bar{\tau}_{\alpha, n n}, \\
\bar{n} \cdot v_{\tau_{\alpha} \tau_{\alpha}}=v_{n, \tau_{\alpha} \tau_{\alpha}}+v_{n} \bar{n} \cdot \bar{n}_{, \tau_{\alpha} \tau_{\alpha}}+2 v_{\tau_{\beta}, \tau_{\alpha}} \bar{\tau}_{\beta, \tau_{\alpha}} \cdot \bar{n}+v_{\tau_{\beta}} \bar{\tau}_{\beta, \tau_{\alpha} \tau_{\alpha}} \cdot \bar{n} .
\end{gathered}
$$

Hence

$$
\begin{aligned}
\left.\bar{n} \cdot \Delta v\right|_{S}= & v_{n, n n}+2 v_{\tau_{\alpha}, n} \bar{n} \cdot \bar{\tau}_{\alpha, n}+v_{\tau_{\alpha}} \bar{n} \cdot \bar{\tau}_{\alpha, n n} \\
& +2 v_{\tau_{\beta}, \tau_{\alpha}} \bar{\tau}_{\beta, \tau_{\alpha}} \cdot \bar{n}+v_{\tau_{\beta}} \bar{\tau}_{\beta, \tau_{\alpha} \tau_{\alpha}} \cdot \bar{n}+\bar{n} \cdot v_{, n} \Delta n+\bar{n} \cdot v_{, \tau_{\alpha}} \Delta \tau_{\alpha},
\end{aligned}
$$

where we used $(1.1)_{3}$.

In the curvilinear coordinates boundary conditions $(1.1)_{4}$ assume the form

$$
v_{n, \tau_{\alpha}}+v_{\tau_{\alpha}, n}-v_{i} n_{i, \tau_{\alpha}}-v_{i} \tau_{\alpha i, n}+\gamma v_{\tau_{\alpha}}=0 .
$$

Using $(1.1)_{3}$ and differentiating (1.13) with respect to $\tau_{\beta}$ yields 


$$
v_{\tau_{\alpha}, n \tau_{\beta}}-\left(v_{i} n_{i, \tau_{\alpha}}+v_{i} \tau_{\alpha i, n}\right)_{, \tau_{\beta}}+\gamma v_{\tau_{\alpha}, \tau_{\beta}}=0
$$

where $\alpha, \beta=1,2$. Applying the curvilinear coordinates we obtain

$$
\operatorname{div} v \equiv v_{n, n}+v_{\tau_{\alpha}, \tau_{\alpha}}+v_{n} \operatorname{div} \bar{n}+v_{\tau_{\alpha}} \operatorname{div} \bar{\tau}_{\alpha}=0 .
$$

Hence

$$
(\operatorname{div} v)_{{ }_{, n}}=v_{n, n n}+v_{\tau_{\alpha}, \tau_{\alpha} n}+\left(v_{n} \operatorname{div} \bar{n}+v_{\tau_{\alpha}} \operatorname{div} \bar{\tau}_{\alpha}\right)_{, n}=0 .
$$

In view of (1.14) and (1.16) the expression (1.12) assumes the form

$$
\begin{aligned}
\left.\bar{n} \cdot \Delta v\right|_{S}= & -\left(v_{i} n_{i, \tau_{\alpha}}+v_{i} \tau_{\alpha i, n}\right)_{,_{\alpha}}+\gamma v_{\tau_{\alpha}, \tau_{\alpha}}-\left[\bar{n} \cdot \nabla, \bar{\tau}_{\alpha} \cdot \nabla\right] v_{\tau_{\alpha}} \\
& -\left(v \operatorname{div} \bar{n}+v_{\tau_{\alpha}} \operatorname{div} \bar{\tau}_{\alpha}\right)_{, n}+2 v_{\tau_{\alpha}, n} \bar{n} \cdot \bar{\tau}{ }_{\alpha, n}+v_{\tau_{\alpha}} \bar{n} \cdot \tau_{\alpha, n n} \\
& +2 v_{\tau_{\beta}, \tau_{\alpha}} \bar{\tau}_{\beta, \tau_{\alpha}} \cdot \bar{n}+v_{\tau_{\beta}} \bar{\tau}_{\beta, \tau_{\alpha} \tau_{\alpha}} \cdot \bar{n}+\bar{n} \cdot v_{, n} \Delta n+\bar{n} \cdot v_{, \tau_{\alpha}} \Delta \tau_{\alpha} \\
\equiv & A_{i j} v_{i, x_{j}}+B_{i} v_{i},
\end{aligned}
$$

where the summation convention over the repeated indices is used and $[\cdot, \cdot]$ denotes the commutator. Finally the last term on the r.h.s. of $(1.10)_{2}$ equals $v_{i} v_{j} n_{j, x_{i}}$.

Summarizing, problem (1.10) takes the form

$$
\begin{array}{ll}
\Delta p=-\nabla v \cdot \nabla v+\operatorname{div} f & \text { in } \Omega, \\
\frac{\partial p}{\partial n}=A_{i j} v_{i, x_{j}}+B_{i} v_{i}+v_{i} v_{j} n_{j, x_{i}}+f \cdot \bar{n} & \text { on } S,
\end{array}
$$

where $A_{i j}, B_{i}$ are defined by (1.17).

1.2. Main results. The main aim of this paper is to prove existence of global regular solutions to problem (1.1) with large initial data and external force. In view of the proofs of existence of global axially symmetric solutions (see [lad 1, ukh 1]) we are able to prove the existence of such solutions to problem (1.1) which remain close to the axially symmetric solutions (see Definition 1.1).

Therefore we distinguish two kinds of quantities: large and small. The large quantities are connected with the corresponding axially symmetric solution and small quantities measure the distance between the solution considered and the axially symmetric solution. The large quantities are: $v_{r}, v_{z}, f_{r}, f_{z}, p, \chi, F_{\varphi}=F_{z}, v_{r}(0), v_{z}(0)$, and the small quantities are: $w=v_{\varphi}, h, f_{\varphi}, \alpha_{r}=\alpha_{1}, \alpha_{z}=\alpha_{2}, F_{r}=F_{1}, F_{z}=F_{2}, q, w(0), h(0)$.

We end this subsection by formulating the main results and outlining the ideas of their proofs. This paper is devoted to proving two theorems: Theorem 1.1 (local existence with large existence time $T$ ) and Theorem 1.3 (global existence, $T \leq \infty$ ).

To formulate the theorems we need the notation:

$$
\begin{aligned}
& f_{r}=f \cdot \bar{e}_{r}, \quad f_{\varphi}=f \cdot \bar{e}_{\varphi}, \quad f_{z}=f \cdot \bar{e}_{z}, \\
& \bar{e}_{r}=(\cos \varphi, \sin \varphi, 0), \quad \bar{e}_{\varphi}=(-\sin \varphi, \cos \varphi, 0), \quad \bar{e}_{z}=(0,0,1), \\
& g=f_{r, \varphi} \bar{e}_{r}+f_{\varphi, \varphi} \bar{e}_{\varphi}+f_{\varphi, z} \bar{e}_{z}, \\
& F=\operatorname{rot} v, \quad F_{1}=F \cdot \bar{e}_{r}, \quad F_{2}=F \cdot \bar{e}_{\varphi}, \quad F_{3}=F \cdot \bar{e}_{z}, \quad F^{\prime}=\left(F_{1}, F_{3}\right), \\
& \alpha=\operatorname{rot} v, \quad \alpha_{1}=\alpha \cdot \bar{e}_{r}, \quad \alpha_{2}=\alpha \cdot \bar{e}_{\varphi}, \quad \alpha_{3}=\alpha \cdot \bar{e}_{z}, \quad \alpha^{\prime}=\left(\alpha_{1}, \alpha_{3}\right), \quad \chi=\alpha_{\varphi}, \\
& w=v_{\varphi}
\end{aligned}
$$

where the dots denote the scalar product in $\mathbb{R}^{3}$. 
Moreover, we introduce the necessary weighted spaces. Let $\nu \in \mathbb{R}$. Then $L_{2, \nu}(Q)$ is the space with the norm $\|u\|_{L_{2, \nu}(Q)}=\left(\int_{Q}|u|^{2} r^{2 \nu} d Q\right)^{1 / 2}, Q \in\left\{\Omega, \Omega^{T}, S, S^{T}\right\}$, and

$$
\begin{aligned}
H_{\nu}^{1}(\Omega) & =\left\{u:\|u\|_{H_{\nu}^{1}(\Omega)}=\left(\int_{\Omega}\left(\left|u_{, x}\right|^{2} r^{2 \nu}+|u|^{2} r^{2(\nu-1)}\right) d x\right)^{1 / 2}<\infty\right\}, \\
W_{2, \nu}^{k}(\Omega) & =\left\{u:\|u\|_{W_{2, \nu}^{k}(\Omega)}=\left(\sum_{|\alpha| \leq k} \int_{\Omega}\left|D_{x}^{\alpha} u(x)\right|^{2} r^{2 \nu} d x\right)^{1 / 2}<\infty\right\}, \\
W_{2, \nu}^{2,1}\left(\Omega^{T}\right) & =\left\{u:\|u\|_{W_{2, \nu}^{2,1}\left(\Omega^{T}\right)}=\left(\int_{\Omega^{T}}\left(\sum_{|\alpha| \leq 2}\left|D_{x}^{\alpha} u\right|^{2}+|u,|^{2}\right) r^{2 \nu} d x d t\right)^{1 / 2}<\infty\right\}, \\
V_{p, \delta}^{l}\left(\Omega^{T}\right) & =\left\{u:\|u\|_{V_{p, \delta}^{l}\left(\Omega^{T}\right)}=\left(\sum_{|\alpha| \leq l} \int_{\Omega^{T}}\left|D_{x}^{\alpha} u\right|^{p} r^{p(\mu-l+|\alpha|)} d x d t\right)^{1 / p}<\infty\right\}
\end{aligned}
$$

and finally

$$
\begin{aligned}
& A_{\nu}\left(\Omega^{T}\right)=\left\{u:\|u\|_{A_{\nu}\left(\Omega^{T}\right)}=\|u\|_{W_{2, \nu}^{2,1}\left(\Omega^{T}\right)}+\left\|u_{, x}\right\|_{W_{2, \nu}^{2,1}\left(\Omega^{T}\right)}<\infty\right\}, \\
& V_{2}^{0}\left(\Omega^{T}\right)=\left\{u:\|u\|_{V_{2}^{0}\left(\Omega^{T}\right)}=\sup _{t \leq T}\|u(t)\|_{L_{2}(\Omega)}+\left(\int_{0}^{T}|\nabla u(x, t)|^{2} d x d t\right)^{1 / 2}<\infty\right\} .
\end{aligned}
$$

Now, we enumerate the assumptions necessary to present the main results.

\section{Assumption A1.}

$$
\begin{aligned}
& f_{\varphi} \in L_{2}\left(\Omega^{T}\right), \quad g \in L_{2,-1}\left(\Omega^{T}\right), \quad F_{1} \in L_{2,-\mu}\left(\Omega^{T}\right), \\
& F_{2} \in L_{2,-1}\left(\Omega^{T}\right), \quad F_{3} \in L_{2}\left(\Omega^{T}\right), \\
& h(0) \in H_{-1}^{1}(\Omega), \quad w(0) \in H_{0}^{1}(\Omega), \quad v(0) \in W_{2,1-\mu}^{2}(\Omega), \quad \alpha^{\prime}(0) \in W_{2,1-\mu}^{1}(\Omega), \\
& \alpha_{1}(0) \in L_{2,-\mu}(\Omega), \quad \chi(0) \in L_{2,-1}(\Omega) \cap H_{1-\mu}^{1}(\Omega), \quad \mu \in(1 / 2,1) .
\end{aligned}
$$

Assumption A2. Let $S^{\prime}$ be a curve described by the equation $\psi(r, z)=0, r \in[0, R]$, $z \in[-a, a]$, where $\psi(r, z)$ is a sufficiently regular function such that $\psi(R, z)=R$ for $z$ from some neighbourhood of $z=0$ and $\psi(0, z)=0$ for $z \in\{-a, a\}$. Let the boundary $S$ be obtained by rotating $S^{\prime}$ around the $x_{3}$ axis.

We also use $r=\psi_{1}(z), z=\psi_{2}(r)$. We assume that $\psi_{1}, \psi_{2}, \psi \in C^{3}$. Let $a_{1}=$ $\psi_{, r} / \sqrt{\psi_{, r}^{2}+\psi_{, z}^{2}}, a_{2}=\psi_{, z} / \sqrt{\psi_{, r}^{2}+\psi_{, z}^{2}}$ be the coordinates of the normal vector to $S$ for $\varphi=$ const.

Let

$$
\begin{aligned}
& \beta_{1}=\frac{k}{r} a_{2}+\frac{1}{r}\left(a_{2, r}+a_{1, z}\right) a_{2}+\frac{a_{1} a_{2}}{r}, \quad \beta_{2}=-\frac{k}{r} a_{1}-\frac{1}{r}\left(a_{2, r}+a_{1, z}\right) a_{1} \\
& \beta_{3}=\left(\frac{a_{1}}{r}-\frac{\gamma}{\nu}\right) a_{2}-\bar{\tau}_{2} \cdot \nabla a_{1}+\left(a_{2, r}-a_{1, z}\right) a_{1}+\frac{a_{1} a_{2}}{r}, \\
& \beta_{4}=-\left(\frac{a_{1}}{r}-\frac{\gamma}{\nu}\right) a_{1}-\bar{\tau}_{2} \cdot \nabla a_{2}+\left(a_{2, r}-a_{1, z}\right) a_{2}+\frac{a_{2}^{2}}{r}, \\
& \beta_{5}=\bar{\tau}_{2} \cdot \nabla\left(\frac{a_{1}}{r}-\frac{\gamma}{\nu}\right)+\frac{1}{r}\left(\bar{n} \cdot \nabla a_{2}-\frac{a_{1} a_{2}}{r}\right),
\end{aligned}
$$

where $\bar{n}=a_{1} \bar{e}_{r}+a_{2} \bar{e}_{z}, \bar{\tau}_{2}=a_{2} \bar{e}_{r}-a_{1} \bar{e}_{z}$ and $k$ is the curvature of $S^{\prime}$. 
Assumption A3.

$$
\begin{aligned}
& \left|\frac{a_{1}}{r}\right|+\left|\nabla \frac{a_{1}}{r}\right| r+\left|\nabla^{2} \frac{a_{1}}{r}\right| r^{2} \leq c, \\
& \left|\beta_{i}\right| r^{3-\mu}+\left|\nabla \beta_{i}\right| r^{4-\mu} \leq c, \quad i=1,2, \\
& \left|\beta_{j}\right|+\left|\nabla \beta_{j}\right| r \leq c, \quad j=3,4, \\
& \left|\beta_{5}\right| r+\left|\nabla \beta_{5}\right| r^{2} \leq c .
\end{aligned}
$$

Let $S_{2}=\left\{x \in S: a_{2} \neq 0\right\}$. Then

$$
\left|\frac{a_{1}}{a_{2}}\right| r^{2}+\left|\nabla \frac{a_{1}}{a_{2}}\right| r \leq c \quad \text { on } S_{2} .
$$

In a neighbourhood of $r=0$ on $S$ :

1. either $\gamma / r-a_{1} / r \geq 0$ or $\left|a_{1}\right| \leq c r$ on $S$,

2. either $0<a_{1}<c r^{2}$ or $a_{1}<0$ and $\left|a_{1}\right| \leq c r$.

Let us introduce the quantities

$$
\begin{aligned}
\bar{X}_{1}(t) & =\|g\|_{L_{2,-1}\left(\Omega^{t}\right)}+\left\|f_{\varphi}\right\|_{L_{2,-\mu}\left(\Omega^{t}\right)}+\left\|F^{\prime}\right\|_{L_{2}\left(\Omega^{t}\right)}+\left\|F_{1}\right\|_{L_{2,-\mu}\left(\Omega^{t}\right)}, \\
\bar{X}_{2}(t) & =\|h(0)\|_{H_{-1}^{1}(\Omega)}+\|w(0)\|_{H_{0}^{1}(\Omega)}+\left\|\alpha^{\prime}(0)\right\|_{W_{2,1-\mu}^{1}(\Omega)}+\left\|\alpha_{1}(0)\right\|_{L_{2,-\mu}(\Omega)}, \\
Y_{1}(t) & =\left\|F_{2}\right\|_{L_{2,-1}\left(\Omega^{t}\right)}+\|\chi(0)\|_{L_{2,-1}(\Omega)}, \\
Y_{2}(t) & =\left\|F_{2}\right\|_{L_{2,1-\mu}\left(\Omega^{t}\right)}+\|\chi(0)\|_{H_{1-\mu}^{1}(\Omega)}+\|v(0)\|_{W_{2,1-\mu}^{2}(\Omega)}, \\
\bar{X}(t) & =\bar{X}_{1}(t)+\bar{X}_{2}(t), \quad \mu \in(1 / 2,1) .
\end{aligned}
$$

Assumption A4. There exist positive constants $A, B$ and positive increasing functions $\varphi_{1}$ and $\varphi_{2}$ such that

$$
\begin{aligned}
& \varphi_{1}(A, B) e^{c t} \bar{X}(t) \leq 1, \\
& c\left[Y_{1}\left(Y_{1}+1\right)^{7 / 4}+Y_{1} Y_{2}+Y_{1}+Y_{2}\right] \leq A, \\
& c Y_{1} \leq B,
\end{aligned}
$$

and for some $\delta \geq 1$ (close to 1 ) the quantity $\delta_{0}=\|k-\gamma /(2 \nu)\|_{V_{4,-\delta / 2}^{3 / 2}\left(S^{t}\right)}$ is so small that

$$
\delta_{0} \leq c\left[Y_{1}\left(Y_{1}+1\right)^{7 / 4}+Y_{1} Y_{2}+Y_{1}+Y_{2}\right]^{-1} .
$$

THEOREM 1.1. Let assumptions A1-A4 hold. Then there exists a unique solution to problem (1.1) such that $v \in A_{1-\mu}\left(\Omega^{t}\right), \chi / r \in V_{2}^{0}\left(\Omega^{t}\right)$, and

$$
\|v\|_{A_{1-\mu}\left(\Omega^{t}\right)} \leq A, \quad\|\chi / r\|_{V_{2}^{0}\left(\Omega^{t}\right)} \leq B,
$$

for all $t \leq T$ where $T \leq \frac{1}{c} \ln \frac{1}{\varphi_{1}(A, B) \bar{X}(T)}$.

Let us introduce the quantities

$$
\begin{aligned}
& \gamma(t) \equiv\|g(t)\|_{L_{2,-1}(\Omega)}+\left\|g_{, t}(t)\right\|_{L_{2}(\Omega)}, \\
& \gamma_{1}(t) \equiv\left\|f_{\varphi}(t)\right\|_{L_{2}(\Omega)}, \\
& \gamma_{2}(t) \equiv\left\|f^{\prime}(t)\right\|_{L_{2,1-\mu}(\Omega)}+\left\|F^{\prime}(t)\right\|_{L_{2}(\Omega)}+\left\|F_{1}(t)\right\|_{L_{2,-\mu}(\Omega)}, \\
& \gamma_{3}(t) \equiv\left\|F_{\varphi}(t)\right\|_{L_{2,-1}(\Omega)}, \\
& \gamma_{4}(t) \equiv\|f(t)\|_{L_{2}(\Omega)},
\end{aligned}
$$


Assumption A5. The external force satisfies the decay estimates

$$
\gamma(t) \leq \gamma(0) e^{-\nu_{0} t}, \quad \gamma_{i}(t) \leq \gamma_{i}(0) e^{-\nu_{i} t}, \quad 1, \ldots, 4,
$$

where $\nu_{0}, \ldots, \nu_{4}$ are positive given numbers.

Introduce the quantities

$$
\begin{aligned}
d_{1}(t) & \equiv\|h(t)\|_{H_{-1}^{1}(\Omega)}+\left\|h_{, t}(t)\right\|_{L_{2}(\Omega)}+\gamma(t)+\gamma_{1}(t), \\
d_{2}(t) & \equiv d_{1}(t)+\gamma_{2}(t), \\
d_{3}(t) & \equiv d_{2}(t)+\gamma_{3}(t)+\gamma_{4}(t), \\
d_{*} & \equiv\|w(0)\|_{L_{2,1}(\Omega)}+\varphi(A) d_{1}(0),
\end{aligned}
$$

where $A$ is the constant from (1.20) and $\varphi$ is some increasing positive function.

Assumption A6. There exist positive constants $A_{1}, A_{2}, A_{3}$ such that for all $t_{0}<T$,

$$
\begin{aligned}
& \|w(0)\|_{H_{0}^{1}(\Omega)} \leq A_{1}, \quad \varphi(A)\left[d_{*}+d_{1}(0)+e^{-\nu T} A_{1}\right] \leq A_{1}, \\
& \varphi(A)\left[e^{-\nu_{*} t_{0}} d_{1}(0)+d_{1}(0)+e^{-\nu T} A_{1}\right]+c \gamma_{2}(0) e^{-\nu T} \equiv A_{2}, \\
& \|\chi(0)\|_{L_{2,-1}(\Omega)} \leq A_{3}, \quad \varphi(A)\left[d_{*}+d_{3}(0)+e^{-T} A_{1}\right]+\gamma_{3}(0)+c \delta_{0} A+e^{-\nu T} A_{3} \leq A_{3},
\end{aligned}
$$

where $\nu_{*}=\min \left\{\nu_{0}, \nu_{1}\right\}$.

THEOREM 1.2. Let $T$ be sufficiently large and let assumptions A1-A6 hold. Then there exists a constant $D$, independent of $k$, such that

$$
\begin{aligned}
\|h(k T)\|_{H_{-1}^{1}(\Omega)}+\left\|h_{, t}(k T)\right\|_{L_{2}(\Omega)} & +\|w(k T)\|_{H_{0}^{1}(\Omega)} \\
& +\|\chi(k T)\|_{L_{2,-1}(\Omega)}+\|\alpha(k T)\|_{W_{2,1-\mu}^{1}(\Omega)} \leq D
\end{aligned}
$$

for all $k \in \mathbb{N}$.

From Theorems 1.1 and 1.2 we have

Theorem 1.3 (global existence). Let the assumptions of Theorems 1.1 and 1.2 hold. Then there exists a global solution to problem (1.1) and constants $A$ and $B$, independent of $k$, such that

$$
\begin{aligned}
& \|v\|_{A_{1-\mu}(\Omega \times(k T,(k+1) T))} \leq A=A(k T,(k+1) T), \\
& \|\chi\|_{V_{2}^{0}(\Omega \times(k T,(k+1) T))} \leq B=B(k T,(k+1) T),
\end{aligned}
$$

for all $k \in \mathbb{N}$, where $A(k T,(k+1) T), B(k T,(k+1) T)$ are the corresponding constants from Theorem 1.1 in the interval $(k T,(k+1) T)$.

1.3. Outline of proof of the main results. Now we describe the proofs of the main results of this paper. Global existence of solutions to problem (1.1) is proved in two steps. First we prove local existence (see Sections 6 and 7 ) by the method of successive approximations. In Lemmas 6.1 and 6.2 we show that the constructed sequence is uniformly bounded.

Next in Theorem 7.7 we prove convergence. In Sections 3, 4, 5 we obtain estimates necessary for the proofs of Lemmas $6.1,6.2$, Theorem 7.7 and the theorem of global existence. 
In Section 4 we obtain estimates for the vorticity vector. The main estimate is the estimate for $\alpha_{\varphi}=\chi$. The estimate depends on the quantity $\delta_{0} \equiv\|k-\gamma / 2\|_{V_{4,-1}^{3 / 2}(S)}$ which must be small for the proofs of local and global existence. This quantity captures the main difference between [zaj 5] and this paper. In [zaj 5] the domain $\Omega$ is a cylinder so $k=0$ and only the case with $\gamma=0$ is considered. In Section 5 we find estimates for the azimuthal component of velocity $w=v_{\varphi}$.

Since the proof of existence of a global axially symmetric solution is based on the estimate for the vorticity vector ( $\operatorname{see}[\operatorname{lad} 1$, uky]) we also derive the main estimates from the problems for vorticity (see Lemmas 4.1, 4.4-4.6). For this we obtain appropriate boundary conditions in Lemma 3.2. The conditions are such that $\left.\alpha\right|_{S}$ depends on $\left.v\right|_{S}$ but $(\bar{n} \cdot \alpha)_{, n}$ is calculated in terms of $\left.v\right|_{S}$ and $\left.v_{, x}\right|_{S}$.

Since $v$ is calculated from the elliptic problem (1.9), the vorticity vector $\alpha$ has one $x$-derivative less than $v$. Therefore, the above boundary conditions for vorticity imply estimates for velocity via interpolation inequalities and an energy type estimate for velocity.

We underline that in the case of the nonslip boundary condition (1.3) we are not able to derive appropriate boundary conditions for $\alpha$.

The main reason why we are able to prove global existence of solutions to problem (1.1) which are close to the axially symmetric solution is that the equation for $\chi$ (see $(1.8)$ ) is only one equation which is nonlinear with respect to large quantities. For this equation we are able to obtain a global estimate for large quantities in terms of nonlinear expressions of norms of small quantities (see Lemma 4.1). Other equations and problems are linear with respect to large quantities and nonlinear with respect to small ones (see (1.4), (1.5), (1.7), (1.9)). Note that the problem for pressure (1.10) is nonlinear with respect to large quantities but to obtain the main estimates in this paper we do not need any estimate for pressure.

Although problem $(1.1)$ for $(v, p)$ is equivalent to problems $(1.7),(1.8),(1.9),(1.10)$ for $(\alpha, v, p)$ (see [zaj 5]), to show existence by the method of successive approximations formulated in Section 6 we also need problems (1.4), (1.5) for $(h, w)$. This is connected with the fact that we are able to get some additional regularity of $(h, w)$ (see Lemmas 4.2 , $4.7,5.1-5.5)$ compared to the regularity of $v$.

In this paper we need two kinds of estimates: energy type estimates and estimates in either $H_{1-\mu}^{2,1}\left(\Omega^{T}\right)$ or $W_{2,1-\mu}^{2,1}\left(\Omega^{T}\right)$, where $\mu \in(1 / 2,1)$. The difference between $H_{1-\mu}^{2,1}\left(\Omega^{T}\right)$ and $W_{2,1-\mu}^{2,1}\left(\Omega^{T}\right)$ is that elements of $H_{1-\mu}^{2,1}\left(\Omega^{T}\right)$ vanish on the axis of symmetry but elements of $W_{2,1-\mu}^{2,1}\left(\Omega^{T}\right)$ do not. Therefore the spaces $H_{1-\mu}^{2,1}\left(\Omega^{T}\right)$ and $W_{2,1-\mu}^{2,1}\left(\Omega^{T}\right)$ describe the behaviour of solutions in a neighbourhood of the axis of symmetry. The use of such spaces is very natural for axially symmetric solutions because the main estimate for $\chi$ derived by Ladyzhenskaya [lad 1] and Ukhovskiı̌-Yudovich [uky] is in weighted Sobolev spaces (see also Lemma 4.1).

The difference between $H_{1-\mu}^{2,1}\left(\Omega^{T}\right)$ and $W_{2,1-\mu}^{2,1}\left(\Omega^{T}\right)$ is examined in Section 2.

The weighted spaces are very appropriate to describe solutions which are close to axially symmetric ones. Therefore in this paper $h \in H_{-1}^{2,1}\left(\Omega^{T}\right)$ (Lemma 4.2), $\alpha^{\prime}=\left(\alpha_{1}, \alpha_{3}\right) \in$ $W_{2,1-\mu}^{2,1}\left(\Omega^{T}\right)\left(\right.$ Lemma 4.3), $\alpha_{1} \in H_{1-\mu}^{2,1}\left(\Omega^{T}\right)\left(\right.$ Lemma 4.4), $\chi \in L_{\infty}\left(0, T ; L_{2,-1}(\Omega)\right) \cap$ $L_{2}\left(0, T ; H_{-1}^{1}(\Omega)\right)$ (Lemma 4.1), $w \in H_{1-\mu}^{2,1}\left(\Omega^{T}\right)$ (Lemma 5.1). 
In [zaj 5] we proved the existence of solutions to the heat equation for the Dirichlet and Neumann problems in weighted Sobolev spaces.

Finally, we have energy estimates for: $h$ (Lemma 3.6), $\chi$ (Lemma 4.1), $\alpha^{\prime}=\left(\alpha_{1}, \alpha_{3}\right)$ (Lemmas 4.4, 4.5), w (Lemmas 5.2-5.6).

To show an energy estimate for $h$ we need the Korn inequality (Lemma 3.4).

Finally, we have to stress that to close all estimates in Section 6 we need a very strong restriction on the shape of $S:\|k-\gamma /(2 \nu)\|_{V_{4,-1 / 2}^{3 / 2}(S)}$ must be sufficiently small. We hope that employing the $L_{p}$-approach, this restriction might be relaxed. 


\section{Notation and auxiliary results}

In this part a simplified notation for different weighted Sobolev spaces is introduced. Moreover the Hardy inequality and some imbedding theorems for weighted Sobolev spaces are formulated. Next we introduce trace spaces of functions from the weighted Sobolev spaces and prove direct and inverse trace theorems. Finally, we formulate results on existence of solutions with corresponding estimates for initial-boundary value problems for the heat equation, nonstationary Stokes system and for the boundary value problem for the (rot, div) elliptic system. Since our considerations are restricted to the $L_{2}$-approach the above existence results are obtained by applying the Fourier-Laplace transforms and examining corresponding problems with parameters.

2.1. Spaces and notation. To simplify the writing we set

$$
\begin{array}{lll}
|u|_{p, Q}=\|u\|_{L_{p}(Q)}, & Q \in\left\{\Omega, S, \Omega^{T}, S^{T}\right\}, & p \in[1, \infty], \\
\|u\|_{s, Q}=\|u\|_{H^{s}(Q)}, & Q \in\{\Omega, S\}, & s \in \mathbb{N} \cup\{0\}, \\
\|u\|_{s, Q}=\|u\|_{W_{2}^{s, s / 2}(Q)}, & Q \in\left\{\Omega^{T}, S^{T}\right\}, & s \in \mathbb{N} \cup\{0\},
\end{array}
$$

where $\|u\|_{0, Q}=|u|_{2, Q}$. We introduce weighted spaces with weight equal to the distance to the axis of symmetry,

$$
\|u\|_{L_{p, \mu}(Q)}=\left(\int_{Q}|u|^{p} r^{p \mu} d Q\right)^{1 / p}, \quad p \in[1, \infty), \mu \in \mathbb{R}, Q \in\left\{\Omega, S, \Omega^{T}, S^{T}\right\},
$$

where $\Omega \subset \mathbb{R}^{3}$ has an axis of symmetry and $r$ is the distance from it in cylindrical coordinates and for simplicity we define

$$
|u|_{p, \mu, Q}=\|u\|_{L_{p, \mu}(Q)} .
$$

Next

$$
\|u\|_{H_{\mu}^{s}(Q)}=\left(\sum_{|\alpha| \leq s} \int_{Q}\left|D_{x}^{\alpha} u\right|^{2} r^{2(\mu-s+|\alpha|)} d Q\right)^{1 / 2},
$$

where $Q \in\{\Omega, S\}, s \in \mathbb{N} \cup\{0\}, \mu \in \mathbb{R}$, and

$$
\|u\|_{H_{\mu}^{s, s / 2}(Q)}=\left(\sum_{|\alpha|+2 i \leq s} \int_{Q}\left|D_{x}^{\alpha} \partial_{t}^{i} u\right|^{2} r^{2(\mu-s+|\alpha|+2 i)} d Q\right)^{1 / 2},
$$

where $Q \in\left\{\Omega^{T}, S^{T}\right\}, s \in \mathbb{N} \cup\{0\}, \mu \in \mathbb{R}$. Moreover, for simplicity, we set

$$
\|u\|_{s, \mu, Q}= \begin{cases}\|u\|_{H_{\mu}^{s}(Q)}, & Q \in\{\Omega, S\}, s \in \mathbb{N} \cup\{0\}, \mu \in \mathbb{R}, \\ \|u\|_{H_{\mu}^{s, s / 2}(Q)}, & Q \in\left\{\Omega^{T}, S^{T}\right\}, s \in \mathbb{N} \cup\{0\}, \mu \in \mathbb{R} .\end{cases}
$$


We define

$$
\|u\|_{l, r, Q}= \begin{cases}\|u\|_{W_{r}^{l, l / 2}(Q)} & \text { for } Q \in\left\{\Omega^{T}, S^{T}\right\} \\ \|u\|_{W_{r}^{l}(Q)} & \text { for } Q \in\{\Omega, S\} .\end{cases}
$$

$$
\begin{gathered}
|u|_{p_{1}, p_{2}, \Omega^{T}}=\|u\|_{L_{p_{2}}\left(0, T ; L_{p_{1}}(\Omega)\right)}, \quad|u|_{p_{1}, p_{2}, \mu, \Omega^{T}}=\|u\|_{L_{p_{2}}\left(0, T ; L_{p_{1}, \mu}(\Omega)\right)}, \\
\|u\|_{W_{p, \mu}^{s}(\Omega)}=\left(\sum_{|\alpha| \leq s} \int_{\Omega}\left|D_{x}^{\alpha} u\right|^{p} r^{p \mu} d x\right)^{1 / p},
\end{gathered}
$$

and

Moreover

$$
\|u\|_{s, p, \mu, \Omega}=\|u\|_{W_{p, \mu}^{s}(\Omega)}, \quad\|u\|_{s, p, \mu, \Omega^{T}}=\|u\|_{W_{p, \mu}^{s, s / 2}\left(\Omega^{T}\right)} .
$$

$$
\|u\|_{L_{p_{1}, p_{2}}\left(\Omega^{T}\right)}=\left(\int_{0}^{T}\left(\int_{\Omega}|u|^{p_{1}} d x\right)^{p_{2} / p_{1}} d t\right)^{1 / p_{2}} .
$$

We also introduce the space $V_{p, \mu}^{l}(Q), Q \in\{\Omega, S\}$, with the norm

$$
\|u\|_{V_{p, \mu}^{l}(Q)}=\left(\sum_{|\alpha| \leq l} \int_{Q}\left|D_{x}^{\alpha} u\right|^{p} r^{p(\mu-l+|\alpha|)} d Q\right)^{1 / p},
$$

where $\mu \in \mathbb{R}, p \in[1, \infty)$. Furthermore, we set

$$
\|u\|_{l, p, \mu, Q^{T}}=\|u\|_{V_{p, \mu}^{l, l / 2}\left(Q^{T}\right)}, \quad\|u\|_{l, p, \mu, Q}=\|u\|_{V_{p, \mu}^{l}(Q)},
$$

where $Q \in\{\Omega, S\}, l \in \mathbb{N} \cup\{0\}, p \in[1, \infty), \mu \in \mathbb{R}$. Finally, we introduce

$$
\|u\|_{A_{\mu}\left(\Omega^{T}\right)}=\|u\|_{W_{2, \mu}^{2,1}\left(\Omega^{T}\right)}+\left\|u_{, x}\right\|_{W_{2, \mu}^{2,1}\left(\Omega^{T}\right)},
$$

and

$$
\|u\|_{V_{2}}^{0}\left(\Omega^{T}\right) \equiv|u|_{\Omega^{T}}=\sup _{t \leq T}|u(t)|_{2, \Omega}+\left(\int_{0}^{T}\left|u_{, x}(t)\right|_{2, \Omega}^{2} d t\right)^{1 / 2} .
$$

2.2. Imbedding and trace theorems. For the reader's convenience we list the estimates and imbeddings which are used in this paper. First,

$$
\begin{gathered}
\|u(t)\|_{s, \Omega} \leq c\left(\|u\|_{s+1, \Omega^{t}}+\|u(0)\|_{s, \Omega}\right), \quad s \in \mathbb{N} \cup\{0\}, \\
\|u(t)\|_{s, \mu, \Omega} \leq c\left(\|u\|_{s+1, \mu, \Omega^{t}}+\|u(0)\|_{s, \mu, \Omega}\right), \quad s \in \mathbb{N} \cup\{0\}, \mu \in \mathbb{R},
\end{gathered}
$$

where $c$ does not depend on $t$.

By $c(\alpha), \varphi(\alpha)$ we denote generic functions which are always positive and increasing and satisfy

$$
|c(\alpha)| \leq c \alpha^{a}, \quad c>0 .
$$

From [bin, Ch. 1, Sect. 2.15] we have the Hardy inequality

$$
|f|_{q,-(1 / q+\alpha), \mathbb{R}_{+}} \leq\left(\frac{\mu}{|\alpha|}\right)^{\mu}\left|f_{, x}\right|_{p, 1-(1 / p+\alpha), \mathbb{R}_{+}},
$$

where $\alpha \neq 0,1 \leq p \leq q \leq \infty, \mu=1-1 / p+1 / q$, and for $p=1, q=\infty$ we assume that $(\mu /|\alpha|)^{\mu}=1$. 
More useful for us is the following Hardy inequality [bin, Ch. 1, Sect. 2.15]:

$$
\left|x^{-\alpha} f\right|_{p, \mathbb{R}_{+}} \leq \frac{1}{|\alpha-1 / p|}\left|x^{-\alpha+1} f_{, x}\right|_{p, \mathbb{R}_{+}}, \quad \alpha \neq \frac{1}{p} .
$$

Using (2.4) we obtain the Hardy inequality for the domain $\Omega$. For this purpose we write (2.4) in the form

$$
\int_{0}^{\infty} r^{-p \alpha}|f|^{p} d r \leq \frac{1}{|\alpha-1 / p|^{p}} \int_{0}^{\infty} r^{-p(\alpha-1)}\left|f_{, r}\right|^{p} d r .
$$

Using cylindrical coordinates we obtain from (2.5) the inequality

$$
\int_{-a}^{a} d z \int_{0}^{2 \pi} d \varphi \int_{0}^{\infty} r^{-p \alpha-1}|f|^{p} r d r \leq \frac{1}{|\alpha-1 / p|^{p}} \int_{-a}^{a} d z \int_{0}^{2 \pi} d \varphi \int_{0}^{\infty} r^{-p(\alpha-1)-1}\left|f_{, r}\right|^{p} r d r .
$$

Hence

$$
|f|_{p,-\mu, \Omega} \leq \frac{1}{|\alpha-1 / p|}\left|f_{, x}\right|_{p, 1-\mu, \Omega},
$$

where $\mu=\alpha+1 / p$.

We also need the imbedding theorem for weighted Sobolev spaces (see Lemma 1.5 in [map 1])

$$
\|u\|_{V_{q, \beta+s-l+n / p-n / q}^{s}(\Omega)} \leq c\|u\|_{V_{p, \beta}^{l}(\Omega)}, \quad \Omega \subset \mathbb{R}^{n},
$$

whenever $s-l+n / p-n / q \leq 0$.

Now we introduce some weighted Sobolev spaces, isotropic and anisotropic, with fractional derivatives. We formulate for them trace theorems, both direct and inverse. We shall do it in the case of $n$ space dimensions, although in this paper the case $n=3$ is considered only.

The space $V_{p, \mu}^{l}(\Omega), \Omega \subset \mathbb{R}^{n}$, has the property that $D_{x}^{\beta} u, u \in V_{p, \mu}^{l}(\Omega)$, vanishes on the axis of symmetry if $|\beta| \leq l-\mu-2 / p$. Therefore to examine functions which do not vanish on the axis of symmetry we introduce the space $W_{p, \mu}^{l}(\Omega)$ with the norm

$$
\|u\|_{W_{p, \mu}^{l}(\Omega)}=\left(\sum_{|\alpha| \leq l} \int_{\Omega}\left|D_{x}^{\alpha} u(x)\right|^{p} r^{p \mu} d x\right)^{1 / p} .
$$

Let us consider a more general anisotropic space $W_{p, \mu}^{\bar{l}}\left(\mathbb{R}^{n+1} ; M\right)$, where $M=\mathbb{R}^{n-2}$ is described by $x^{\prime}=\left(x_{1}, x_{2}\right)=0$, and $\bar{l}=\left(l_{0}, l_{1}, \ldots, l_{n}\right), l_{i} \in \mathbb{N}, i=0, \ldots, n, p \in[1, \infty]$, $\mu \in \mathbb{R}$, and

$$
\|u\|_{W_{p, \mu}^{i}\left(\mathbb{R}^{n+1} ; M\right)}=\sum_{i=0}^{n}\left\|\partial_{x_{i}}^{l_{i}} u\right\|_{L_{p, \mu}\left(\mathbb{R}^{n+1} ; M\right)}+\|u\|_{L_{p, \mu}\left(\mathbb{R}^{n+1} ; M\right)},
$$

and

$$
\|u\|_{L_{p, \mu}\left(\mathbb{R}^{n+1} ; M\right)}=\left(\int_{\mathbb{R}^{n+1}}|u(\bar{x})|^{p}\left|x^{\prime}\right|^{p \mu} d \bar{x}\right)^{1 / p},
$$

where $\bar{x}=\left(x_{0}, x_{1}, \ldots, x_{n}\right), x=\left(x_{1}, \ldots, x_{n}\right), x^{\prime}=\left(x_{1}, x_{2}\right)$.

From [zaj 3] we have 
Lemma 2.0. Assume that $u \in W_{p, \mu}^{\bar{l}}(Q), Q \in \mathbb{R}^{n+1}, \bar{l}=\left(l_{0}, l_{1}, \ldots, l_{n}\right), 1<p<q<\infty$, $\mu, \nu \in \mathbb{R}_{+}, 0<l_{i} \in \mathbb{Z}, 0 \leq \sigma_{i} \in \mathbb{Z}, i=0,1, \ldots, n, l_{1}=l_{2}=l_{*}$,

$$
\varkappa=1-\left(\frac{1}{p}-\frac{1}{q}\right) \sum_{i=0}^{n} \frac{1}{l_{i}}-\sum_{i=0}^{n} \frac{\sigma_{i}}{l_{i}}-\frac{1}{l_{*}}(\mu-\nu) \geq 0,
$$

and $q$ satisfies the $R(\bar{l})$-horn condition. Assume $\mu>\nu$. Then $D^{\bar{\sigma}} u \in L_{q, \nu}(Q), \bar{\sigma}=$ $\left(\sigma_{0}, \sigma_{1}, \ldots, \sigma_{n}\right)$, and

$$
\left\|D^{\bar{\sigma}} u\right\|_{L_{q, \nu}(Q)} \leq c_{1} \delta^{\varkappa}\|u\|_{L_{p, \mu}^{\bar{l}}(Q)}+c_{2} \delta^{\varkappa-1}\|u\|_{L_{p, \mu}(Q)}
$$

for all $\delta \in\left(0, h_{0}\right)$, where the constants $c_{1}, c_{2}$ do not depend on $f$ and $h_{0}=h_{0}(Q)$.

First we consider the space $V_{p, \mu}^{k}\left(\mathbb{R}_{+}^{n} ; M\right), k \in \mathbb{N}, p \in(1, \infty), \mu \in \mathbb{R}, M=\mathbb{R}^{n-2}$ is determined by $x^{\prime}=\left(x_{1}, x_{2}\right)=0$ and $\mathbb{R}_{+}^{n}=\left\{x \in \mathbb{R}^{n}: x_{n}>0\right\}$. For the weight we take the power function $r^{\mu}$, where $r=\left|x^{\prime}\right|$.

We underline that the space (and other spaces introduced later) has a different structure than the spaces used for elliptic (or parabolic) problems in domains with edges because the weight is calculated from a subspace intersecting the domain and orthogonal to the boundary (see [map], [soz]).

By $V_{p, q}^{k-1 / p}\left(\mathbb{R}^{n-1} ; M\right)$ we denote the space of traces on $x_{n}=0$ of functions from $V_{p, \mu}^{k}\left(\mathbb{R}_{+}^{n} ; M\right)$, i.e. $V_{p, \mu}^{k-1 / p}\left(\mathbb{R}^{n-1} ; M\right)=V_{p, \mu}^{k}\left(\mathbb{R}_{+}^{n} ; M\right) \backslash \stackrel{\circ}{V_{p, \mu}^{k}}\left(\mathbb{R}_{+}^{n} ; M\right)$, where $\stackrel{\circ}{V}_{p, \mu}^{k}\left(\mathbb{R}_{+}^{n} ; M\right)$ is the closure of the smooth functions vanishing on $\partial \mathbb{R}_{+}^{n}$. Therefore

$$
\|u\|_{V_{p, \mu}^{k-1 / p}\left(\mathbb{R}^{n-1} ; M\right)}=\inf \left\{\|v\|_{V_{p, \mu}^{k}\left(\mathbb{R}_{+}^{n} ; M\right)}: v-u \in \stackrel{\circ}{V}_{p, \mu}^{k}\left(\mathbb{R}_{+}^{n} ; M\right)\right\} .
$$

From Lemma 1.3 of [map] we have

LEMMA 2.1. The norm in $V_{p, \mu}^{k-1 / p}\left(\mathbb{R}^{n-1} ; M\right)$ is equivalent to

$$
\begin{aligned}
\left(\left.\int_{\mathbb{R}^{n-1}} \int_{\mathbb{R}^{n-1}} \sum_{|\alpha|=k-1}|| \bar{x}_{1}^{\prime}\right|^{\mu} D_{\bar{x}_{1}}^{\alpha} u\left(\bar{x}_{1}\right)-\left.\left|\bar{x}_{2}^{\prime}\right|^{\mu} D_{\bar{x}_{2}}^{\alpha} u\left(\bar{x}_{2}\right)\right|^{p} \frac{d \bar{x}_{1} d \bar{x}_{2}}{\left|\bar{x}_{1}-\bar{x}_{2}\right|^{n+p-2}}\right. \\
+\int_{\mathbb{R}^{n-1}|\alpha| \leq k-1} \sum_{\left.\left|\alpha \bar{x}^{\prime} u(\bar{x})\right|^{p}\left|\bar{x}^{\prime}\right|^{p(\mu-k+|\alpha|)-1}\right)^{1 / p}}
\end{aligned}
$$

where $\bar{x}=\left(x_{1}, x_{2}, \ldots, x_{n-1}\right), \bar{x}^{\prime}=\left(x_{1}, x_{2}\right)$.

Proof. Let $W_{p}^{k-1 / p}\left(\mathbb{R}^{n-1}\right)$ be the space of traces on $x_{n}=0$ of functions from $W_{p}^{k}\left(\mathbb{R}^{n}\right)$. For $u$ such that $\operatorname{supp} u \subset\left\{x \in \mathbb{R}^{n-1}: 1<\left|x^{\prime}\right|<2\right\}$ we have the equivalence

$$
c_{1}\|u\|_{V_{p, \mu}^{k-1 / p}\left(\mathbb{R}^{n-1} ; M\right)} \leq\|u\|_{W_{p}^{k-1 / p}\left(\mathbb{R}^{n-1}\right)} \leq c_{2}\|u\|_{V_{p, \mu}^{k-1 / p}\left(\mathbb{R}^{n-1} ; M\right)},
$$

where

$$
\begin{array}{r}
\|u\|_{W_{p}^{k-1 / p}\left(\mathbb{R}^{n-1}\right)}=\left(\int_{\mathbb{R}^{n-1}} \int_{\mathbb{R}^{n-1}} \sum_{|\alpha|=k-1}\left|D_{x_{1}}^{\alpha} u\left(x_{1}\right)-D_{x_{2}}^{\alpha} u\left(x_{2}\right)\right|^{p} \frac{d x_{1} d x_{2}}{\left|x_{1}-x_{2}\right|^{n+p-2}}\right. \\
\left.+\int_{\mathbb{R}^{n-1}} \sum_{|\alpha| \leq k-1}\left|D_{x}^{\alpha} u(x)\right|^{p} d x\right)^{1 / p},
\end{array}
$$

and in (2.13) the norm $\|u\|_{V_{p, \mu}^{k-1 / p}\left(\mathbb{R}^{n-1} ; M\right)}$ has the form (2.12). 
Using the homothety $x \mapsto 2^{\nu} x$ we find that for $u$ such that supp $u \subset\left\{x: 2^{\nu-1}<\right.$ $\left.\left|x^{\prime}\right|<2^{\nu+1}\right\}$ the norms $V_{p, \mu}^{k-1 / p}\left(\mathbb{R}^{n-1} ; M\right)$ and $(2.12)$ are equivalent. Let us introduce a partition of unity $\left\{\zeta_{\nu}\right\}_{\nu=-\infty}^{\infty}$ such that $\operatorname{supp} \zeta_{\nu} \subset\left\{x \in \mathbb{R}^{n}: 2^{\nu-1}<\left|x^{\prime}\right|<2^{\nu+1}\right\}$ and $\left|D_{x}^{\alpha} \zeta_{\nu}(x)\right| \leq c 2^{-|\alpha| \nu}, \nu=0, \mp 1, \ldots$.

Lemma 1.1 in [map] implies that the norm (2.11) is equivalent to the norm

$$
\left(\sum_{\nu=-\infty}^{\infty}\left\|\zeta_{\nu} u\right\|_{V_{p, \mu}^{k-1 / p}\left(\mathbb{R}^{n-1} ; M\right)}\right)^{1 / p}
$$

Using this equivalence we end the proof.

Although the norm (2.12) for traces is commonly used, it is more convenient for us to employ norms which distinguish directions orthogonal and parallel to $M$. This happens when we consider anisotropic spaces for space-time dependent functions. For this purpose we use [iln], where the following result has been shown:

LEMma 2.2. The norm of $W_{p}^{k-1 / p}\left(\mathbb{R}^{n-1}\right)$ is equivalent to the norm

$$
\begin{aligned}
\left(\int_{\mathbb{R}^{2}} d \bar{x}^{\prime} \sum_{|\alpha|=k-1} \int_{\mathbb{R}^{n-3}} \int_{\mathbb{R}^{n-3}}\left|D_{\bar{x}_{1}^{\prime \prime}}^{\alpha} u\left(\bar{x}^{\prime}, \bar{x}_{1}^{\prime \prime}\right)-D_{\bar{x}_{2}^{\prime \prime}}^{\alpha} u\left(\bar{x}^{\prime}, \bar{x}_{2}^{\prime \prime}\right)\right|^{p} \frac{d \bar{x}_{1}^{\prime \prime} d \bar{x}_{2}^{\prime \prime}}{\left|\bar{x}_{1}^{\prime \prime}-\bar{x}_{2}^{\prime \prime}\right|^{n+p-4}}\right. \\
\quad+\int_{\mathbb{R}^{n-3}} d \bar{x}^{\prime \prime} \sum_{|\alpha|=k-1} \iint_{\mathbb{R}^{2}}\left|D_{\mathbb{R}^{2}}^{\alpha} u\left(\bar{x}_{1}^{\prime}, \bar{x}^{\prime \prime}\right)-D_{\bar{x}_{2}^{\prime}}^{\alpha} u\left(\bar{x}_{2}^{\prime}, \bar{x}^{\prime \prime}\right)\right|^{p} \frac{d \bar{x}_{1}^{\prime} d \bar{x}_{2}^{\prime}}{\left|\bar{x}_{1}^{\prime}-\bar{x}_{2}^{\prime}\right|^{p+1}} \\
\left.\quad+\sum_{|\alpha| \leq k-1} \int_{\mathbb{R}^{n-1}}\left|D_{\bar{x}}^{\alpha} u(\bar{x})\right|^{p} d \bar{x}\right)^{1 / p},
\end{aligned}
$$

where $\bar{x}=\left(x_{1}, \ldots, x_{n-1}\right), \bar{x}^{\prime}=\left(x_{1}, x_{2}\right), \bar{x}^{\prime \prime}=\left(x_{3}, \ldots, x_{n-1}\right)$.

From Lemma 1.4 in [map] we have

LEMma 2.3. The norm of $V_{p, \mu}^{k-1 / p}\left(\mathbb{R}^{n-1} ; M\right)$ is equivalent to the norm

$$
\begin{aligned}
& \left(\int_{\mathbb{R}^{2}}\left|\bar{x}^{\prime}\right|^{p \mu} d \bar{x}^{\prime} \sum_{|\alpha|=k-1} \int_{\mathbb{R}^{n-3}} \int_{\mathbb{R}^{n-3}}\left|D_{\bar{x}_{1}^{\prime \prime}}^{\alpha} u\left(\bar{x}^{\prime}, \bar{x}_{1}^{\prime \prime}\right)-D_{\bar{x}_{2}^{\prime \prime}}^{\alpha} u\left(\bar{x}^{\prime}, \bar{x}_{2}^{\prime \prime}\right)\right|^{p} \frac{d \bar{x}_{1}^{\prime \prime} d \bar{x}_{2}^{\prime \prime}}{\left|\bar{x}_{1}^{\prime \prime}-\bar{x}_{2}^{\prime \prime}\right|^{n+p-4}}\right. \\
& +\left.\int_{\mathbb{R}^{n-3}} d \bar{x}^{\prime \prime} \sum_{|\alpha|=k-1} \int_{\mathbb{R}^{2}} \int_{\mathbb{R}^{2}}|| \bar{x}_{1}^{\prime}\right|^{\mu} D_{\bar{x}_{1}^{\prime}}^{\alpha} u\left(\bar{x}_{1}^{\prime}, \bar{x}^{\prime \prime}\right)-\left.\left|\bar{x}_{2}^{\prime}\right|^{\mu} D_{\bar{x}_{2}^{\prime}}^{\alpha} u\left(\bar{x}_{2}^{\prime}, \bar{x}^{\prime \prime}\right)\right|^{p} \frac{d \bar{x}_{1}^{\prime} d \bar{x}_{2}^{\prime}}{\left|\bar{x}_{1}^{\prime}-\bar{x}_{2}^{\prime}\right|^{p+1}} \\
& \left.+\sum_{|\alpha| \leq k-1} \int_{\mathbb{R}^{n-1}}\left|D_{\bar{x}}^{\alpha} u(\bar{x})\right|^{p}\left|\bar{x}^{\prime}\right|^{p(\mu-k+|\alpha|)-1}\right)^{1 / p} \cdot
\end{aligned}
$$

The proof is similar to the proof of Lemma 2.1 .

Now we pass to the anisotropic space $V_{p, \mu}^{k, k / 2}\left(\mathbb{R}_{+}^{n} \times(0, T) ; M\right), k \in \mathbb{N}, p \in(1, \infty)$, $\mu \in \mathbb{R}$. By $V_{p, \mu}^{k-1 / p, k / 2-1 /(2 p)}\left(\mathbb{R}^{n-1} \times(0, T) ; M\right)$ we denote the space of traces on $x_{n}=0$ of functions from $V_{p, \mu}^{k, k / 2}\left(\mathbb{R}_{+}^{n} \times(0, T) ; M\right)$, i.e. $V_{p, \mu}^{k-1 / p, k / 2-1 /(2 p)}\left(\mathbb{R}^{n-1} \times(0, T) ; M\right)=$ $V_{p, \mu}^{k, k / 2}\left(\mathbb{R}_{+}^{n} \times(0, T) ; M\right) \backslash \stackrel{\circ}{V} k, k / 2\left(\mathbb{R}_{+}^{n} \times(0, T) ; M\right)$, where $\stackrel{\circ}{V}_{p, \mu}^{k, k}\left(\mathbb{R}_{+}^{n} \times(0, T) ; M\right)$ is equal to the closure of the smooth functions vanishing on $\partial \mathbb{R}_{+}^{n} \times(0, T), \partial \mathbb{R}_{+}^{n}=\left\{x \in \mathbb{R}^{n}: x_{n}=0\right\}$. 
Therefore

$$
\begin{gathered}
\|u\|_{V_{p, \mu}^{k-1 / p, k / 2-k /(2 p)}\left(\mathbb{R}^{n-1} \times(0, T) ; M\right)}=\inf \left\{\|v\|_{V_{p, \mu}^{k, k / 2}\left(\mathbb{R}_{+}^{n} \times(0, T) ; M\right)}:\right. \\
\left.v-u \in \stackrel{\circ}{V}_{p, \mu}^{k, k / 2}\left(\mathbb{R}_{+}^{n} \times(0, T) ; M\right)\right\} .
\end{gathered}
$$

Similarly to Lemma 2.3 we have

LEMMA 2.4. The norm of $V_{p, \mu}^{k-1 / p, k / 2-1 / 2 p}\left(\mathbb{R}^{n-1} \times(0, T) ; M\right)$ is equivalent to the norm

$$
\begin{aligned}
& \left(\int _ { \mathbb { R } ^ { 2 } } | \overline { x } ^ { \prime } | ^ { p \mu } d \overline { x } ^ { \prime } \sum _ { | \alpha | + 2 a = k - 1 } \left[\int_{0}^{T} d t \int_{\mathbb{R}^{n-3}} \int_{\mathbb{R}^{n-3}} \mid D_{\bar{x}_{1}^{\prime \prime}}^{\alpha} \partial_{t}^{a} u\left(\bar{x}^{\prime}, \bar{x}_{1}^{\prime \prime}, t\right)\right.\right. \\
& \quad-\left.D_{\bar{x}_{2}^{\prime \prime}}^{\alpha} \partial_{t}^{a} u\left(\bar{x}^{\prime}, \bar{x}_{2}^{\prime \prime}, t\right)\right|^{p} \frac{d \bar{x}_{1}^{\prime \prime} d \bar{x}_{2}^{\prime \prime}}{\left|\bar{x}_{1}^{\prime \prime}-\bar{x}_{2}^{\prime \prime}\right|^{n+p-4}} \\
& \left.\quad+\int_{\mathbb{R}^{n-3}} d \bar{x}^{\prime \prime} \int_{0}^{T} \int_{0}^{T}\left|D_{\bar{x}^{\prime \prime}}^{\alpha} \partial_{t}^{a} u\left(\bar{x}^{\prime}, \bar{x}^{\prime \prime}, t\right)-D_{\bar{x}^{\prime \prime}}^{\alpha} \partial_{t^{\prime}}^{a} u\left(\bar{x}^{\prime}, \bar{x}^{\prime \prime}, t^{\prime}\right)\right|^{p} \frac{d t d t^{\prime}}{\left|t-t^{\prime}\right|^{1 / 2+p / 2}}\right] \\
& +\left.\int_{\mathbb{R}^{n-1}} d \bar{x}^{\prime \prime} \int_{0}^{T} d t \sum_{|\alpha|+2 a=k-1} \int_{\mathbb{R}^{2}} \int_{\mathbb{R}^{2}}|| \bar{x}_{1}^{\prime}\right|^{\mu} D_{\bar{x}_{1}^{\prime}}^{\alpha} u\left(\bar{x}_{1}^{\prime}, \bar{x}^{\prime \prime}, t\right)-\left.\left|\bar{x}_{2}^{\prime}\right|^{\mu} D_{\bar{x}_{2}^{\prime}}^{\alpha} u\left(\bar{x}_{2}^{\prime}, \bar{x}^{\prime \prime}, t\right)\right|^{p} \\
& \left.\quad \cdot \frac{d \bar{x}_{1}^{\prime} d \bar{x}_{2}^{\prime}}{\left|\bar{x}_{1}^{\prime}-\bar{x}_{2}^{\prime}\right|^{p+1}}+\left.\sum_{|\alpha|+2 a \leq k-1} \int_{0}^{T}\left|D_{\mathbb{R}^{n-1}}\right| D_{\bar{x}}^{\alpha} \partial_{t}^{a} u(\bar{x}, t)\right|^{p}\left|\bar{x}^{\prime}\right|^{p(\mu-k+|\alpha|+2 a)-1}\right)^{1 / p} .
\end{aligned}
$$

Finally, we introduce the traces for $t=0$ which belong to the space $V_{p, \mu}^{k-2 / p}\left(\mathbb{R}_{+}^{n} ; M\right)$. A lemma similar to Lemma 2.4 can be formulated.

Applying homothety (see the proof of Lemma 2.1) we can prove the following trace results (see $[\operatorname{map}])$ :

Lemma 2.5. Assume that $u \in V_{p, \mu}^{k, k / 2}\left(\mathbb{R}_{+}^{n} \times(0, T) ; M\right), k,|\alpha|, a \in \mathbb{N}, k-|\alpha|-2 a \geq 1$. Then $\left.D_{x}^{\alpha} \partial_{t}^{a} u\right|_{x_{n}=0} \in V_{p, \mu}^{k-|\alpha|-2 a-1 / p, k / 2-|\alpha| / 2-a-1 /(2 p)}\left(\mathbb{R}^{n-1} \times(0, T) ; M\right)$ and

$$
\begin{aligned}
\left\|\left.D_{x}^{\alpha} \partial_{t}^{a} u\right|_{x_{n}=0}\right\|_{V_{p, \mu}^{k-|\alpha|-2 a-1 / p, k / 2-|\alpha| / 2-a-1 /(2 p)}\left(\mathbb{R}^{n-1} \times(0, T) ; M\right)} & \leq c\|u\|_{V_{p, \mu}^{k, k / 2}\left(\mathbb{R}_{+}^{n} \times(0, T) ; M\right)} \\
& \leq .
\end{aligned}
$$

Assume that $\varphi_{j} \in V_{p, \mu}^{k-j-1 / p, k / 2-j / 2-1 /(2 p)}\left(\mathbb{R}^{n-1} \times(0, T) ; M\right), j \leq k-1$, are given. Then there exists a function $u \in V_{p, \mu}^{k, k / 2}\left(\mathbb{R}_{+}^{n} \times(0, T) ; M\right)$ such that

$$
\left.\frac{\partial^{j} u}{\partial x_{n}^{j}}\right|_{x_{n}=0}=\varphi_{j}, \quad j=0, \ldots, k-1
$$

and

$$
\|u\|_{V_{p, \mu}^{k, k / 2}\left(\mathbb{R}_{+}^{n} \times(0, T) ; M\right)} \leq \sum_{j=0}^{k-1}\left\|\varphi_{j}\right\|_{V_{p, \mu}^{k-j-1 / p, k / 2-j / 2-1 /(2 p)}\left(\mathbb{R}^{n-1} \times(0, T) ; M\right)} .
$$

To simplify considerations we omit the parameter $M$ in the definitions of norms introduced below. 
By $L_{p, \mu}^{k}\left(\mathbb{R}_{+}^{n}\right), L_{p, \mu}^{k-1 / p}\left(\mathbb{R}^{n-1}\right), W_{p, \mu}^{k-1 / p}\left(\mathbb{R}^{n-1}\right), \mathbb{R}^{n-1}=\left.\mathbb{R}_{+}^{n}\right|_{x_{n}=0}, k \in \mathbb{N}, p \in[1, \infty]$, $\mu \in \mathbb{R}$, we define sets of functions for which the following expressions are finite:

$$
\begin{gathered}
\|u\|_{L_{p, \mu}^{k}\left(\mathbb{R}_{+}^{n}\right)}=\left(\sum_{|\alpha|=k} \int_{\mathbb{R}_{+}^{n}}\left|D_{x}^{\alpha} u(x)\right|^{p}\left|x^{\prime}\right|^{p \mu} d x\right)^{1 / p}, \quad x^{\prime}=\left(x_{1}, x_{2}\right), \\
\|u\|_{L_{p, \mu}^{k-1 / p}\left(\mathbb{R}^{n-1}\right)}=\left(\sum_{|\alpha|=k-1} \int_{\mathbb{R}^{n-1}}\left|x^{\prime}\right|^{p \mu} d x \int_{K_{+}(x)} \frac{\left|D^{\alpha} u(x)-D^{\alpha} u(x+y)\right|^{p}}{|y|^{n+p-2}} d y\right)^{1 / p},
\end{gathered}
$$

where $K_{+}(x)=\left\{y \in \mathbb{R}^{n-1}:|y|<\left|x^{\prime}\right|\right\}$, and

$$
\|u\|_{W_{p, \mu}^{k-1 / p}\left(\mathbb{R}^{n-1}\right)}=\left(\sum_{|\alpha| \leq k-1} \int_{\mathbb{R}^{n-1}}\left|D_{x}^{\alpha} u(x)\right|^{p}\left|x^{\prime}\right|^{p \mu} d x\right)^{1 / p}+\|u\|_{L_{p, \mu}^{k-1 / p}\left(\mathbb{R}^{n-1}\right)} .
$$

Similarly we introduce the anisotropic spaces

$$
W_{p, \mu}^{k-1 / p, k / 2-1 /(2 p)}\left(\mathbb{R}^{n-1} \times(0, T)\right), \quad L_{p, \mu}^{k-1 / p, k / 2-1 /(2 p)}\left(\mathbb{R}^{n-1} \times(0, T)\right) .
$$

From $[\mathrm{soz}]$ we have

LEMMA 2.6. Let $u \in L_{p, \mu}^{k}\left(\mathbb{R}_{+}^{n}\right)$ and $|\alpha|<k$. Then $\left.D^{\alpha} u\right|_{x_{n}=0} \in L_{p, \mu}^{k-|\alpha|-1 / p}\left(\mathbb{R}^{n-1}\right)$ and

$$
\left\|\left.D^{\alpha} u\right|_{x_{n}=0}\right\|_{L_{p, \mu}^{k-|\alpha|-1 / p}\left(\mathbb{R}^{n-1}\right)} \leq c\|u\|_{L_{p, \mu}^{k}\left(\mathbb{R}_{+}^{n}\right)} .
$$

Let $u \in W_{p, \mu}^{k}\left(\mathbb{R}_{+}^{n}\right)$ and $|\alpha|<k$. Then

$$
\left.D^{\alpha} u\right|_{x_{n}=0} \in W_{p, \mu}^{k-|\alpha|-1 / p}\left(\mathbb{R}^{n-1}\right)
$$

and

$$
\left\|\left.D^{\alpha} u\right|_{x_{n}=0}\right\|_{W_{p, \mu}^{k-|\alpha|-1 / p}\left(\mathbb{R}^{n-1}\right)} \leq c\|u\|_{W_{p, \mu}^{k}\left(\mathbb{R}_{+}^{n}\right)} .
$$

Lemma 2.7. Let $\varphi_{j} \in L_{p, \mu}^{k-j-1 / p}\left(\mathbb{R}^{n-1}\right), i=0, \ldots, k-1$, be given. Then there exists a function $u \in L_{p, \mu}^{k}\left(\mathbb{R}_{+}^{n}\right)$ such that

$$
\left.\frac{\partial^{j} u}{\partial x_{n}^{j}}\right|_{x_{n}=0}=\varphi_{j}, \quad j=0, \ldots, k-1
$$

and

$$
\|u\|_{L_{p, \mu}^{k}\left(\mathbb{R}_{+}^{n}\right)} \leq c \sum_{j=0}^{k-1}\left\|\varphi_{j}\right\|_{L_{p, \mu}^{k-j-1 / p}\left(\mathbb{R}^{n-1}\right)} .
$$

Let $\varphi_{j} \in W_{p, \mu}^{k-j-1 / p}\left(\mathbb{R}^{n-1}\right), j=0, \ldots, k-1$, be given. Then there exists a function $u^{\prime} \in W_{p, \mu}^{k}\left(\mathbb{R}_{+}^{n}\right)$ such that

$$
\left.\frac{\partial^{j} u^{\prime}}{\partial x_{n}^{j}}\right|_{x_{n}=0}=\varphi_{j}, \quad j=0, \ldots, k-1
$$

and

$$
\left\|u^{\prime}\right\|_{W_{p, \mu}^{k}\left(\mathbb{R}_{+}^{n}\right)} \leq c \sum_{j=0}^{k-1}\left\|\varphi_{j}\right\|_{W_{p, \mu}^{k-j-1 / p}\left(\mathbb{R}^{n-1}\right)} .
$$

Similar results hold for anisotropic Sobolev spaces. 
Now we find a relation between $V_{p, \mu}^{k}\left(\mathbb{R}_{+}^{n}\right)$ and $W_{p, \mu}^{k}\left(\mathbb{R}_{+}^{n}\right)$. Assume that $u \in L_{p, \mu}^{k}\left(\mathbb{R}_{+}^{n}\right)$, $1-2 / p+s-1<\mu<1-2 / p+s, k>s$. Then by the Hardy inequality

$$
\left\|u-P^{k-s-1}(u)\right\|_{L_{p, \mu-k}^{0}\left(\mathbb{R}_{+}^{n}\right)} \leq c\|u\|_{L_{p, \mu}^{k}\left(\mathbb{R}_{+}^{n}\right)},
$$

where

$$
P^{\sigma}(u)=\left.\sum_{i=0}^{\sigma} \frac{\partial^{i} u}{\partial r^{i}}\right|_{r=0} \frac{r^{i}}{i !} .
$$

Therefore if $u \in W_{p, \mu}^{k}\left(\mathbb{R}_{+}^{n}\right)$ then $u-P^{k-s-1}(u) \in V_{p, \mu}^{k}\left(\mathbb{R}_{+}^{n}\right)$ and

$$
\left\|u-P^{k-s-1}(u)\right\|_{V_{p, \mu}^{k}\left(\mathbb{R}_{+}^{n}\right)} \leq c\|u\|_{W_{p, \mu}^{k}\left(\mathbb{R}_{+}^{n}\right)} .
$$

Now we recall some Hardy inequalities.

Lemma 2.8 (see [kon]). Assume that $u \in L_{2, \mu}^{m}(\Omega), \Omega \subset \mathbb{R}^{3}, \Omega$ is an axially symmetric domain. Assume that $s>\mu>s-1, m, s \in \mathbb{N} \cup\{0\}, m \geq s$. Then

$$
\left\|u-P^{m-s-1}(u)\right\|_{L_{2, \mu-m}(\Omega)} \leq c\|u\|_{L_{2, \mu}^{m}(\Omega)},
$$

where

$$
P^{k}(u)=\left.\sum_{i \leq k} u^{(i)}\right|_{r=0} \frac{r^{i}}{i !}
$$

and $u^{(i)}=\partial^{i} u / \partial r^{i}$.

Proof. Let $m=1, s=0$. Taking $u \in L_{2, \mu}^{1}(\Omega)$ and using cylindrical coordinates we have

$$
\int_{-a}^{a} d z \int_{0}^{2 \pi} d \varphi \int_{0}^{\psi_{1}(z)} r^{2 \mu+1}\left|\frac{\partial u}{\partial r}\right|^{2} d r<\infty
$$

where $\Omega=\left\{x \in \mathbb{R}^{3}: z \in(-a, a), \varphi \in(0,2 \pi), r<\psi_{1}(z)\right\}$. To apply the Hardy inequality it is enough to consider only the integral

$$
I=\int_{0}^{R} r^{2 \mu+1}\left|\frac{\partial u}{\partial r}\right|^{2} d r .
$$

Since $\mu \in(-1,0)$ Lemma 4.2 from [kon] implies that $u$ is a continuous function of $r$. Therefore Lemma 4.10 from [kon] implies

$$
I \geq\left. c \int_{0}^{R} r^{2 \mu-1}|u-u|_{r=0}\right|^{2} d r .
$$

Let $m \in \mathbb{N}$ and $s=0$. Then in view of the above considerations we have

$$
\begin{aligned}
I_{1} & =\int_{0}^{R} r^{2 \mu+1}\left|\frac{\partial^{m} u}{\partial r^{m}}\right|^{2} d r \geq\left. c \int_{0}^{R} r^{2 \mu-1}\left|\frac{\partial^{m-1} u}{\partial r^{m-1}}-\frac{\partial^{m-1} u}{\partial r^{m-1}}\right|_{r=0}\right|^{2} d r \\
& =c \int_{0}^{R} r^{2 \mu-1}\left|\frac{\partial^{m-1}}{\partial r^{m-1}}\left(u-\left.u^{(m-1)}\right|_{r=0} \frac{r^{m-1}}{(m-1) !}\right)\right|^{2} d r \\
& \geq c \int_{0}^{R} r^{2 \mu-3} \mid \frac{\partial^{m-2}}{\partial r^{m-2}}\left(u-\left.u^{(m-1)}\right|_{r=0} \frac{r^{m-1}}{(m-1) !}\right)
\end{aligned}
$$




$$
\begin{aligned}
& -\left.\left.\frac{\partial^{m-2}}{\partial r^{m-2}}\left(u-\left.u^{(m-1)}\right|_{r=0} \frac{r^{m-1}}{(m-1) !}\right)\right|_{r=0}\right|^{2} d r \\
= & \left.c \int_{0}^{R} r^{2 \mu-3}\left|\frac{\partial^{m-2}}{\partial r^{m-2}}\left(u-\left.u^{(m-1)}\right|_{r=0} \frac{r^{m-1}}{(m-1) !}\right)-\frac{\partial^{m-2}}{\partial r^{m-2}} u\right|_{r=0}\right|^{2} d r \\
= & c \int_{0}^{R} r^{2 \mu-3}\left|\frac{\partial^{m-2}}{\partial r^{m-2}}\left(u-\left.u^{(m-1)}\right|_{r=0} \frac{r^{m-1}}{(m-1) !}-\left.u^{(m-2)}\right|_{r=0} \frac{r^{m-2}}{(m-2) !}\right)\right|^{2} d r \\
\geq & \cdots \geq c \int_{0}^{R} r^{2 \mu+1-2 m}\left|u-P^{m-1}(u)\right|^{2} d r .
\end{aligned}
$$

Continuing the procedure for $s>0$ we conclude the proof.

From [bin, Ch. 1, Sect. 2.16] we have the Hardy inequality:

Lemma 2.9. Let $p \in[1, \infty], \beta \neq 1 / p, F(x)=\int_{0}^{x} f(y) d y$ for $\beta>1 / p$ and $F(x)=$ $\int_{x}^{\infty} f(y) d y$ for $\beta<1 / p$. Then

$$
\left|x^{-\beta} F\right|_{p, \mathbb{R}_{+}^{1}} \leq \frac{1}{|\beta-1 / p|}\left|x^{-\beta+1} f\right|_{p, \mathbb{R}_{+}^{1}} .
$$

From Lemmas 2.8 and 2.9 we have

Lemma 2.10. Assume that $u \in L_{p, \mu}^{m}(\Omega), p \in[1, \infty)$, and $\Omega \subset \mathbb{R}^{3}$ is an axially symmetric domain. Assume that $1-2 / p+s-1<\mu<1-2 / p+s, m, s \in \mathbb{N} \cup\{0\}, m \geq s+1$. Then

$$
\left\|u-P^{m-s-1}(u)\right\|_{L_{p, \mu-m}(\Omega} \leq \frac{c}{|1-2 / p-\mu|}\|u\|_{L_{p, \mu}^{m}(\Omega)},
$$

where the polynomial $P^{k}$ is defined in Lemma 2.8 .

Proof. We shall restrict our considerations to the case $m=1$ because the case $m>1$ can be considered in the same way as in the proof of Lemma 2.8. Taking $u \in L_{p, \mu}^{1}(\Omega)$ and using cylindrical coordinates we have

$$
\int_{-a}^{a} d z \int_{0}^{2 \pi} d \varphi \int_{0}^{\psi_{1}(z)} r^{p \mu+1}\left|\frac{\partial u}{\partial r}\right|^{p} d r<\infty .
$$

To apply the Hardy inequality we consider the integral

$$
I=\int_{0}^{\psi_{1}(z)} r^{p \mu+1}\left|\frac{\partial u}{\partial r}\right|^{p} d r .
$$

Applying the Hardy inequality (2.23) with $\beta=1-1 / p-\mu$ we obtain

$$
I \geq c \int_{0}^{\psi_{1}(z)} r^{p(\mu-1)+1}|u-u(0)|^{p} d r
$$

if $1-2 / p-\mu \neq 0$. Following the proof of Lemma 2.8 we finish the proof. 
2.3. Estimates for some elliptic and parabolic problems. We need

Lemma 2.11. Assume that $u \in W_{p}^{2,1}(\Omega \times(0, T)), \Omega \subset \mathbb{R}^{n}$. Assume that $u$ has traces $u(t), u(0) \in W_{p}^{2-2 / p}(\Omega), t \in[0, T]$. Then there exists a constant $c_{0}$, which does not depend on $T$, such that

$$
\sup _{t \leq T}\|u(t)\|_{W_{p}^{2-2 / p}(\Omega)} \leq c_{0}\left(\|u\|_{W_{p}^{2,1}(\Omega \times(0, T))}+\|u(0)\|_{W_{p}^{2-2 / p}(\Omega)}\right) .
$$

Proof. Let $\widetilde{u}$ be an extension of $u$ such that $\widetilde{u} \in W_{p}^{2,1}\left(\mathbb{R}^{n} \times(0, T)\right)$ and

$$
\|\widetilde{u}\|_{W_{p}^{2,1}\left(\mathbb{R}^{n} \times(0, T)\right)} \leq c\|u\|_{W_{p}^{2,1}(\Omega \times(0, T))} .
$$

In view of the above inequality we have for the trace $u(0)$ a corresponding extension such that $\widetilde{u}(0) \in W_{p}^{2-2 / p}\left(\mathbb{R}^{n}\right)$ and

$$
\|\widetilde{u}(0)\|_{W_{p}^{2-2 / p}\left(\mathbb{R}^{n}\right)} \leq c\|u(0)\|_{W_{p}^{2-2 / p}(\Omega)} .
$$

In the case of bounded $\Omega$, the above extensions can be taken in such a way that supp $\tilde{u} \subset$ $B_{R}$, where $R<\infty, B_{R}$ is a ball of radius $R$ and $\Omega \subset B_{R}$.

Let us introduce a new function $v=\widetilde{u}-\widetilde{u}(0)$. Hence $\left.v\right|_{t=0}=0$. Therefore $v$ can be extended by zero for $t<0$. Denote the extension by $\widetilde{v}$. Then $\widetilde{v} \in W_{p}^{2,1}\left(\mathbb{R}^{n} \times(-\infty, T)\right)$ and

$$
\|\widetilde{v}\|_{W_{p}^{2,1}\left(\mathbb{R}^{n} \times(-\infty, T)\right)} \leq c\|v\|_{W_{p}^{2,1}\left(\mathbb{R}^{n} \times(0, T)\right)} .
$$

Next we extend $\widetilde{v}$ to $t>T$. Denote the extension by $\widetilde{v}$. Hence we have

$$
\begin{aligned}
\|\widetilde{\widetilde{v}}\|_{W_{p}^{2,1}\left(\mathbb{R}^{n} \times \mathbb{R}\right)} & \leq c\|\widetilde{v}\|_{W_{p}^{2,1}\left(\mathbb{R}^{n} \times(-\infty, T)\right)} \\
& \leq c\|v\|_{W_{p}^{2,1}\left(\mathbb{R}^{n} \times(0, T)\right)} \leq c\left(\|\widetilde{u}\|_{W_{p}^{2,1}\left(\mathbb{R}^{n} \times(0, T)\right)}+\|\widetilde{u}(0)\|_{W_{p}^{2-2 / p}\left(\mathbb{R}^{n}\right)}\right) \\
& \leq c\left(\|u\|_{W_{p}^{2,1}(\Omega \times(0, T))}+\|u(0)\|_{W_{p}^{2-2 / p}(\Omega)}\right),
\end{aligned}
$$

where the constants $c$ do not depend on $T$. Taking the trace

$$
\|\widetilde{\widetilde{v}}(t)\|_{W_{p}^{2-2 / p}\left(\mathbb{R}^{n}\right)} \leq c\left(\|u\|_{W_{p}^{2,1}(\Omega \times(0, T))}+\|u(0)\|_{W_{p}^{2-2 / p}(\Omega)}\right),
$$

where $c$ does not depend on $T$, we obtain estimate (2.25). This concludes the proof.

The proof of Lemma 2.11 can also be applied to weighted Sobolev spaces $W_{p, \mu}^{2,1}\left(\Omega^{T}\right)$ because it does not depend on the properties of functions with respect to the $x$ variables. The extension from $\Omega$ to $\mathbb{R}^{n}$ can also be done for functions from weighted Sobolev spaces. Therefore we have

Lemma 2.12. Assume that $u \in W_{p, \mu}^{2,1}(\Omega \times(0, T)), \Omega \subset \mathbb{R}^{n}, \mu \in \mathbb{R}, p \in(1, \infty)$. Assume that $u(t), u(0) \in W_{p, \mu}^{2-2 / p}(\Omega), t \in[0, T]$. Then there exists a constant $c_{0}^{\prime}$, which does not depend on $T$, such that

$$
\sup _{t \leq T}\|u(t)\|_{W_{p, \mu}^{2-2 / p}(\Omega)} \leq c_{0}^{\prime}\left(\|u\|_{W_{p, \mu}^{2,1}(\Omega \times(0, T))}+\|u(0)\|_{W_{p, \mu}^{2-2 / p}(\Omega)}\right) .
$$

In this paper we consider problem (1.1) in the whole axially symmetric domain so behaviour of solutions near the axis of symmetry must be taken into account. To prove existence of solutions to problem (1.1) we need to show existence of solutions of the 
following elliptic and evolution problems. First we examine the elliptic problem

$$
\begin{array}{ll}
\operatorname{rot} v=w & \text { in } \Omega, \\
\operatorname{div} v=0 & \text { in } \Omega, \\
v \cdot \bar{n}=0 & \text { on } S .
\end{array}
$$

From [zaj 2] we have

Lemma 2.13. Assume that $w \in W_{2, \mu}^{2,1}\left(\Omega^{T}\right), \mu \in \mathbb{R}_{+}$. Then there exists a solution to problem (2.27) such that $v, v_{x} \in W_{2, \mu}^{2,1}\left(\Omega^{T}\right)$ and

$$
\|v\|_{A_{\mu}\left(\Omega^{T}\right)} \leq c\|w\|_{W_{2, \mu}^{2,1}\left(\Omega^{T}\right)} .
$$

Let us consider the following problems:

$$
\begin{array}{ll}
u_{, t}-\Delta u=f & \text { in } \Omega^{T}, \\
\left.u\right|_{t=0}=u_{0} & \text { in } \Omega,
\end{array}
$$

with either the Dirichlet boundary conditions

$$
\left.u\right|_{S}=u_{1} \quad \text { on } S^{T}
$$

or the Neumann boundary conditions

$$
\left.\frac{\partial u}{\partial n}\right|_{S}=u_{2} \quad \text { on } S^{T}
$$

From [zaj 1] we have

Lemma 2.14. Assume that $f \in L_{2, \mu}\left(\Omega^{T}\right), u_{0} \in W_{2, \mu}^{1}(\Omega), u_{1} \in W_{2, \mu}^{3 / 2,3 / 4}\left(S^{T}\right), u_{2} \in$ $W_{2, \mu}^{1 / 2,1 / 4}\left(S^{T}\right), \mu \in \mathbb{R}_{+}$. Then there exist solutions to problems (2.29), (2.30) and (2.29), (2.31) such that $u \in W_{2, \mu}^{2,1}\left(\Omega^{T}\right)$ and

$$
\|u\|_{W_{2, \mu}^{2,1}\left(\Omega^{T}\right)} \leq c\left(\|f\|_{L_{2, \mu}\left(\Omega^{T}\right)}+\left\|u_{0}\right\|_{W_{2, \mu}^{1}(\Omega)}+\left\|u_{1}\right\|_{W_{2, \mu}^{3 / 2,3 / 4}\left(S^{T}\right)}\right),
$$

and

$$
\|u\|_{W_{2, \mu}^{2,1}\left(\Omega^{T}\right)} \leq c\left(\|f\|_{L_{2, \mu}\left(\Omega^{T}\right)}+\left\|u_{0}\right\|_{W_{2, \mu}^{1}(\Omega)}+\left\|u_{2}\right\|_{W_{2, \mu}^{1 / 2,1 / 4}\left(S^{T}\right)}\right) .
$$

Finally, we consider the nonstationary Stokes problem

$$
\begin{array}{ll}
v_{, t}-\nu \Delta v+\nabla p=f & \text { in } \Omega^{T}, \\
\operatorname{div} v=0 & \text { in } \Omega^{T}, \\
\left.v\right|_{t=0}=v_{0} & \text { in } \Omega, \\
v \cdot \bar{n}=0 & \text { on } S^{T}, \\
\bar{n} \cdot \mathbb{D}(v) \cdot \bar{\tau}_{\alpha}=0, \quad \alpha=1,2, & \text { on } S^{T} .
\end{array}
$$

Assume that solutions of (2.34) satisfy

$$
\int_{\Omega} v_{\varphi}(r, \varphi, z) d x=0, \quad \int_{\Omega} p d x=0 .
$$

Then we have 
Lemma 2.15. Assume that $v_{0} \in H_{\mu}^{1}(\Omega), f \in L_{2, \mu}\left(\Omega^{T}\right), \mu \in[-1,0)$. Assume (2.35). Then there exists a solution to (2.34) such that $v \in H_{\mu}^{2,1}\left(\Omega^{T}\right)$ and

$$
\|v\|_{H_{\mu}^{2,1}\left(\Omega^{T}\right)}+\|p\|_{L_{2}\left(0, T ; H_{\mu}^{1}(\Omega)\right)} \leq c\left(\left\|v_{0}\right\|_{H_{\mu}^{1}(\Omega)}+\|f\|_{L_{2, \mu}\left(\Omega^{T}\right)}\right) .
$$

2.4. Differential operators in cylindrical coordinates. Finally, we express different differential operators in the cylindrical coordinates $r, \varphi, z$. We recall the relations $x_{1}=$ $r \cos \varphi, x_{2}=r \sin \varphi, x_{3}=z$ and the vectors $\bar{e}_{1}=\bar{e}_{r}=(\cos \varphi, \sin \varphi, 0), \bar{e}_{2}=\bar{e}_{\varphi}=$ $(-\sin \varphi, \cos \varphi, 0), \bar{e}_{3}=\bar{e}_{z}=(0,0,1)$. The Laplace operator in cylindrical coordinates has the form

$$
\Delta u=\frac{1}{r} \partial_{r}\left(r u_{, r}\right)+\frac{1}{r^{2}} u_{, \varphi \varphi}+u_{, z z}=u_{, r r}+\frac{1}{r} u_{, r}+\frac{1}{r^{2}} u_{, \varphi \varphi}+u_{, z z} .
$$

Let $v$ be a vector. Then $\Delta v$ in cylindrical coordinates takes the form

$$
\begin{aligned}
& \Delta v \cdot \bar{e}_{1}=\Delta\left(v_{\alpha} \bar{e}_{\alpha}\right) \cdot \bar{e}_{1}=\Delta v_{r}-\frac{v_{r}}{r^{2}}-\frac{2}{r^{2}} v_{\varphi, \varphi}, \\
& \Delta v \cdot \bar{e}_{2}=\Delta\left(v_{\alpha} \bar{e}_{\alpha}\right) \cdot \bar{e}_{2}=\Delta v_{\varphi}-\frac{v_{\varphi}}{r^{2}}+\frac{2}{r^{2}} v_{r, \varphi}, \\
& \Delta v \cdot \bar{e}_{3}=\Delta v_{z},
\end{aligned}
$$

where $v_{\alpha}=v \cdot \bar{e}_{\alpha}, \alpha=1,2,3$, and the summation convention over repeated indices is assumed.

Let $\mathbb{D}(v)=\left\{d_{i j}(v)\right\}=\left\{v_{i, x_{j}}+v_{j, x_{i}}\right\}$. We calculate

$$
\begin{aligned}
& d_{r r}=d_{i j} e_{r i} e_{r j}=2 v_{r, r}, \quad d_{r \varphi}=d_{i j} e_{r i} e_{\varphi j}=\frac{1}{r} v_{r, \varphi}-\frac{1}{r} v_{\varphi}+v_{\varphi, r}, \\
& d_{\varphi \varphi}=d_{i j} e_{\varphi i} e_{\varphi j}=\frac{2}{r} v_{\varphi, \varphi}+\frac{2 v_{r}}{r}, \quad d_{z r}=d_{i j} e_{z i} e_{r j}=v_{r, z}+v_{z, r} \\
& d_{z \varphi}=d_{i j} e_{z i} e_{\varphi j}=v_{\varphi, z}+\frac{1}{r} v_{z, \varphi}, \quad d_{z z}=2 v_{z, z}
\end{aligned}
$$

Finally,

$$
v \cdot \nabla h \cdot \bar{e}_{r}=v \cdot \nabla h_{r}-\frac{v_{\varphi} h_{\varphi}}{r}, \quad v \cdot \nabla h \cdot \bar{e}_{\varphi}=v \cdot \nabla h_{\varphi}+\frac{v_{\varphi} h_{r}}{r}, \quad v \cdot \nabla h \cdot \bar{e}_{z}=v \cdot \nabla h_{z},
$$

where $v \cdot \nabla h=\left(v_{r} \partial_{r}+\frac{v_{\varphi}}{r} \partial_{\varphi}+\partial_{z}\right) h$. Moreover,

$$
\operatorname{div} v=v_{r, r}+\frac{1}{r} v_{\varphi, \varphi}+v_{z, z}+\frac{v_{r}}{r}
$$

and

$$
\begin{aligned}
& \alpha_{r}=\operatorname{rot} v \cdot \bar{e}_{1}=\frac{1}{r}\left[v_{z, \varphi}-r v_{\varphi, z}\right], \\
& \alpha_{\varphi}=\operatorname{rot} v \cdot \bar{e}_{2}=v_{r, z}-v_{z, r}, \\
& \alpha_{z}=\operatorname{rot} v \cdot \bar{e}_{3}=\frac{1}{r}\left[\left(r v_{\varphi}\right)_{, r}-v_{r, \varphi}\right] .
\end{aligned}
$$




\section{Boundary conditions for velocity and vorticity}

In this section we express the slip boundary condition in cylindrical coordinates (Lemma 3.1 ), we find the boundary conditions for the vorticity vector (Lemma 3.2) and show the Korn inequality necessary to prove energy type estimates for $v$ and $h$.

3.1. Boundary conditions for velocity in cylindrical coordinates. First we express the boundary conditions $(1.1)_{3,4}$ in terms of cylindrical components of velocity.

Lemma 3.1. Assume that $S$ is described by the relation $\psi(r, z)=0$. Let

$$
a_{1}=\frac{\psi_{, r}}{\sqrt{\psi_{, r}^{2}+\psi_{, z}^{2}}}, \quad a_{2}=\frac{\psi_{, z}}{\sqrt{\psi_{, r}^{2}+\psi_{, z}^{2}}} .
$$

Then $(1.1)_{3}$ takes the form

$$
a_{1} v_{r}+a_{2} v_{z}=0
$$

and $(1.1)_{4}$ implies

$$
\begin{aligned}
& a_{1} v_{\varphi, r}+a_{2} v_{\varphi, z}+\frac{\gamma}{\nu} v_{\varphi}=\frac{1}{r} a_{1} v_{\varphi} \\
& 2 a_{1} a_{2}\left(v_{r, r}-v_{z, z}\right)+\left(a_{2}^{2}-a_{1}^{2}\right)\left(v_{r, z}+v_{z, r}\right)+\frac{\gamma}{\nu}\left(a_{2} v_{r}-a_{1} v_{z}\right)=0 .
\end{aligned}
$$

Proof. We have $\nabla \psi=\left(\psi_{, r} \cos \varphi, \psi_{, r} \sin \varphi, \psi_{, z}\right)=\psi_{, r} \bar{e}_{r}+\psi_{, z} \bar{e}_{z}$. Hence

$$
\left.\bar{n}\right|_{S}=a_{1} \bar{e}_{r}+a_{2} \bar{e}_{z},\left.\quad \bar{\tau}_{1}\right|_{S}=\bar{e}_{\varphi},\left.\quad \bar{\tau}_{2}\right|_{S}=a_{2} \bar{e}_{r}-a_{1} \bar{e}_{z} .
$$

Then $(1.1)_{3}$ implies $\psi_{, r} v_{r}+\psi_{, z} v_{z}=0$, so (3.1) holds. The condition (1.1) $)_{4}$ for $j=1$ yields

$$
n_{i}\left(v_{i, x_{j}}+v_{j, x_{i}}\right) \tau_{1 j}+\frac{\gamma}{\nu} v \cdot \bar{\tau}_{1}=0
$$

where the summation convention over repeated indices is assumed. By (3.3) condition (3.4) reads

$$
\frac{1}{r}\left(a_{1} \bar{e}_{r}+a_{2} \bar{e}_{z}\right) \cdot v_{, \varphi}+\left(a_{1} \bar{e}_{r}+a_{2} \bar{e}_{z}\right) \cdot \nabla v_{\varphi}+\frac{\gamma}{\nu} v_{\varphi}=0
$$

So

$$
\frac{1}{r}\left(a_{1} v_{r, \varphi}-a_{1} v_{\varphi}+a_{2} v_{z, \varphi}\right)+a_{1} v_{\varphi, r}+a_{2} v_{\varphi, z}+\frac{\gamma}{\nu} v_{\varphi}=0 .
$$

Using (3.1) and the fact that $a_{1}, a_{2}$ do not depend on $\varphi$ we obtain $(3.2)_{1}$. The condition $(1.1)_{4}$ for $j=2$ amounts to

$$
n_{i}\left(v_{i, x_{j}}+v_{j, x_{i}}\right) \tau_{2 j}+\frac{\gamma}{\nu} v \cdot \bar{\tau}_{2}=0
$$


so in view of (3.3) it implies

$$
\left(a_{1} \bar{e}_{r}+a_{2} \bar{e}_{z}\right) \cdot \nabla v_{i}\left(a_{2} \bar{e}_{r}-a_{1} \bar{e}_{z}\right)_{i}+\left(a_{2} \bar{e}_{r}-a_{1} \bar{e}_{z}\right) \cdot \nabla v_{i}\left(a_{1} \bar{e}_{r}+a_{2} \bar{e}_{z}\right)_{i}+\frac{\gamma}{\nu} v \cdot \bar{\tau}_{2}=0
$$

which gives $(3.2)_{2}$. This concludes the proof.

3.2. Boundary conditions for vorticity. Now we find the boundary conditions for the vorticity vector $\alpha=\operatorname{rot} v$. In cylindrical coordinates we have the relations

$$
\begin{aligned}
\alpha_{1} & =\alpha_{r}=\frac{1}{r} v_{z, \varphi}-v_{\varphi, z}, \\
\alpha_{2}=\alpha_{\varphi} & =v_{r, z}-v_{z, r}, \\
\alpha_{3}=\alpha_{z} & =v_{\varphi, r}+\frac{1}{r} v_{\varphi}-\frac{1}{r} v_{r, \varphi} .
\end{aligned}
$$

LEMMA 3.2. Let the assumptions of Lemma 3.1 hold. Let $S^{\prime}$ be a curve which generates $S$ by rotating around the $z$ axis. Let $S^{\prime}$ be described also by $\psi(r, z)=0$. Let $k$ be the curvature of $S^{\prime}$. Let $\bar{\tau}_{2}$ be the tangent vector to $S$ described by (3.3). Then

$$
\begin{gathered}
\alpha_{2}=2(k-\gamma /(2 \nu)) v \cdot \bar{\tau}_{2} \quad \text { on } S, \\
a_{2} \alpha_{1}-a_{1} \alpha_{3}=-\frac{2 a_{1}}{r} v_{\varphi}+\frac{\gamma}{\nu} v_{\varphi} \quad \text { on } S, \\
\alpha_{n, n}=\beta_{1} v_{r, \varphi}+\beta_{2} v_{z, \varphi}+\beta_{3} v_{\varphi, r}+\beta_{4} v_{\varphi, z}+\beta_{5} v_{\varphi},
\end{gathered}
$$

where $\beta_{i}=\beta_{i}\left(\nabla \psi, \nabla^{2} \psi\right), i=1, \ldots, 5$, are defined by (3.15).

Proof. Since $a_{2} \partial_{r}-a_{1} \partial_{z}$ is the tangent operator to $S$, (3.1) implies

$$
\left(a_{2} \partial_{r}-a_{1} \partial_{z}\right)\left(a_{1} v_{r}+a_{2} v_{z}\right)=0 .
$$

Calculations yield

$$
\begin{aligned}
a_{1} a_{2}\left(v_{r, r}-\right. & \left.v_{z, z}\right)+a_{2}^{2} v_{z, r}-a_{1}^{2} v_{r, z} \\
& =\left(a_{1} a_{1, z}-a_{2} a_{1, r}\right) v_{r}+\left(a_{1} a_{2, z}-a_{2} a_{2, r}\right) v_{z}=-\left(a_{1, r}+a_{2, z}\right) v \cdot \bar{\tau}_{2},
\end{aligned}
$$

where the second equality follows by applying the relation $a_{1}^{2}+a_{2}^{2}=1$, and $\bar{\tau}_{2}$ from (3.3) is used.

Now we find a geometrical description of the r.h.s. of (3.10). Let $r=r(s), z=z(s)$ be a curve in the $(r, z)$ plane. Then its curvature equals

$$
k=\frac{\dot{r} \ddot{z}-\ddot{r} \dot{z}}{\left(\dot{r}^{2}+\dot{z}^{2}\right)^{3 / 2}},
$$

where the dot denotes the derivative with respect to the parameter $s$. If the curve is written in the form $\psi(r, z)=0$, its curvature takes the form

$$
k=\frac{\psi_{, z}^{2} \psi_{, r r}+\psi_{, r}^{2} \psi_{, z z}-2 \psi_{, r} \psi_{, z} \psi_{, r z}}{\left(\psi_{, r}^{2}+\psi_{, z}^{2}\right)^{3 / 2}} .
$$

Using the form of $a_{1}$ and $a_{2}$ we see that

$$
a_{1, r}+a_{2, z}=k .
$$

Now from (3.10), (3.11) and (3.2) 2 we obtain (3.6). 
Using (3.1), (3.2) 1 and (3.5) we calculate

$$
\begin{aligned}
a_{2} \alpha_{1}-a_{1} \alpha_{3} & =\frac{1}{r}\left(a_{2} v_{z, \varphi}+a_{1} v_{r, \varphi}\right)-\left(a_{1} v_{\varphi, r}+a_{2} v_{\varphi, z}\right)-\frac{a_{1}}{r} v_{\varphi} \\
& =-\left(a_{1} v_{\varphi, r}+a_{2} v_{\varphi, z}\right)-\frac{a_{1}}{r} v_{\varphi}=-\frac{2 a_{1}}{r} v_{\varphi}+\frac{\gamma}{\nu} v_{\varphi}
\end{aligned}
$$

which implies (3.7).

Finally, we show (3.8). We have

$$
\alpha_{n}=\alpha \cdot \bar{n}=a_{1} \alpha_{1}+a_{2} \alpha_{3}=a_{1}\left(\frac{1}{r} v_{z, \varphi}-v_{\varphi, z}\right)+a_{2}\left(\frac{1}{r} v_{\varphi}+v_{\varphi, r}-\frac{1}{r} v_{r, \varphi}\right) .
$$

Hence

$$
\begin{aligned}
\alpha_{n, n}= & \left(a_{1} \bar{e}_{r}+a_{2} \bar{e}_{z}\right) \cdot \nabla \alpha_{n}=a_{1} \partial_{r} \alpha_{n}+a_{2} \partial_{z} \alpha_{n} \\
= & a_{1} \partial_{r}\left[a_{1}\left(\frac{1}{r} v_{z, \varphi}-v_{\varphi, z}\right)+a_{2}\left(\frac{1}{r} v_{\varphi}+v_{\varphi, r}-\frac{1}{r} v_{r, \varphi}\right)\right] \\
& +a_{2} \partial_{z}\left[a_{1}\left(\frac{1}{r} v_{z, \varphi}-v_{\varphi, z}\right)+a_{2}\left(\frac{1}{r} v_{\varphi}+v_{\varphi, r}-\frac{1}{r} v_{r, \varphi}\right)\right] \\
= & \left.-a_{1}^{2} v_{\varphi, r z}+a_{1} a_{2} v_{\varphi, r r}-a_{1} a_{2} v_{\varphi, z z}+a_{2}^{2} v_{\varphi, r z}\right] \\
& +\frac{1}{r}\left[a_{1}^{2} v_{z, \varphi r}-a_{1} a_{2} v_{r, \varphi r}+a_{1} a_{2} v_{z, \varphi z}-a_{2}^{2} v_{r, \varphi z}\right] \\
& +a_{1} a_{1, r}\left(\frac{1}{r} v_{z, \varphi}-v_{\varphi, z}\right)+a_{1} a_{2, r}\left(\frac{1}{r} v_{\varphi}+v_{\varphi, r}-\frac{1}{r} v_{r, \varphi}\right) \\
& +a_{2} a_{1, z}\left(\frac{1}{r} v_{z, \varphi}-v_{\varphi, z}\right)+a_{2} a_{2, z}\left(\frac{1}{r} v_{\varphi}+v_{\varphi, r}-\frac{1}{r} v_{r, \varphi}\right)-\frac{a_{1}^{2}}{r^{2}} v_{z, \varphi} \\
& +a_{1} a_{2} \partial_{r}\left(\frac{1}{r} v_{\varphi}\right)+\frac{a_{1} a_{2}}{r^{2}} v_{r, \varphi}+\frac{a_{2}^{2}}{r} v_{\varphi, z} .
\end{aligned}
$$

Now we shall estimate the particular terms on the r.h.s. of (3.12). The first term equals $a_{1}\left(-a_{1} \partial_{z} v_{\varphi, r}+a_{2} \partial_{r} v_{\varphi, r}\right)+a_{2}\left(a_{2} \partial_{r} v_{\varphi, z}-a_{1} \partial_{z} v_{\varphi, z}\right)$
$\quad=a_{1} \bar{\tau}_{2} \cdot \nabla v_{\varphi, r}+a_{2} \bar{\tau}_{2} \cdot \nabla v_{\varphi, z}=\bar{\tau}_{2} \cdot \nabla\left(\bar{n} \cdot \nabla v_{\varphi}\right)-\bar{\tau}_{2} \cdot \nabla a_{1} v_{\varphi, r}-\bar{\tau}_{2} \cdot \nabla a_{2} v_{\varphi, z} \equiv I_{1}$.

Since $(3.2)_{1}$ takes the form

$$
\bar{n} \cdot \nabla v_{\varphi}=-\frac{\gamma}{\nu} v_{\varphi}+\frac{a_{1}}{r} v_{\varphi}
$$

we have

$$
I_{1}=\bar{\tau}_{2} \cdot \nabla\left(\left(\frac{a_{1}}{r}-\frac{\gamma}{\nu}\right) v_{\varphi}\right)-\bar{\tau}_{2} \cdot \nabla a_{1} v_{\varphi, r}-\bar{\tau}_{2} \cdot \nabla a_{2} v_{\varphi, z}
$$

The second term on the r.h.s. of (3.12) takes the form $(1 / r) K_{, \varphi}$, where

$$
K=a_{1}^{2} v_{z, r}-a_{2}^{2} v_{r, z}+a_{1} a_{2}\left(v_{z, z}-v_{r, r}\right) .
$$

Writing $(3.2)_{2}$ in the form

$$
2 a_{1} a_{2}\left(v_{r, r}-v_{z, z}\right)+\left(a_{2}^{2}-a_{1}^{2}\right)\left(v_{r, z}+v_{z, r}\right)=-\frac{\gamma}{\nu} \bar{\tau}_{2} \cdot v
$$


and applying it to eliminate the last expression in $K$ we obtain

$$
K=k v \cdot \bar{\tau}_{2} .
$$

Finally, we examine the last eight terms on the r.h.s. of (3.12). The terms with $v_{r, \varphi}, v_{z, \varphi}$ take the form

$$
\frac{1}{r}\left(a_{2, r}+a_{1, z}\right)\left(\bar{\tau}_{2} \cdot v\right)_{, \varphi}+\frac{a_{1} a_{2}}{r^{2}} v_{r, \varphi} .
$$

The terms with $v_{\varphi, r}, v_{\varphi, z}$ equal

$$
\left(a_{2, r}-a_{1, z}\right) \bar{n} \cdot \nabla v_{\varphi}+\frac{a_{2}}{r} \bar{n} \cdot \nabla v_{\varphi} .
$$

Finally, the remaining term gives the expression

$$
\frac{1}{r}\left(\bar{n} \cdot \nabla a_{2}-\frac{a_{1} a_{2}}{r}\right) v_{\varphi} .
$$

Summarizing,

$$
\begin{aligned}
\alpha_{n, n}= & \bar{\tau}_{2} \cdot \nabla\left[\left(\frac{a_{1}}{r}-\frac{\gamma}{\nu}\right) v_{\varphi}\right]-\bar{\tau}_{2} \cdot \nabla a_{1} v_{\varphi, r}-\bar{\tau}_{2} \cdot \nabla a_{2} v_{\varphi, z} \\
& +\frac{1}{r}\left(k v \cdot \bar{\tau}_{2}\right)_{, \varphi}+\frac{1}{r}\left(a_{2, r}+a_{1, z}\right)\left(\bar{\tau}_{2} \cdot v\right)_{, \varphi}+\frac{a_{1} a_{2}}{r^{2}} v_{r, \varphi} \\
& +\left(a_{2, r}-a_{1, z}\right) \bar{n} \cdot \nabla v_{\varphi}+\frac{a_{2}}{r} \bar{n} \cdot \nabla v_{\varphi}+\frac{1}{r}\left(\bar{n} \cdot \nabla a_{2}-\frac{a_{1} a_{2}}{r}\right) v_{\varphi} \\
\equiv & \beta_{1} v_{r, \varphi}+\beta_{2} v_{z, \varphi}+\beta_{3} v_{\varphi, r}+\beta_{4} v_{\varphi, z}+\beta_{5} v_{\varphi}
\end{aligned}
$$

where

$$
\begin{aligned}
& \beta_{1}=\frac{k}{r} a_{2}+\frac{1}{r}\left(a_{2, r}+a_{1, z}\right) a_{2}+\frac{a_{1} a_{2}}{r}, \\
& \beta_{2}=-\frac{k}{r} a_{1}-\frac{1}{r}\left(a_{2, r}+a_{1, z}\right) a_{1}, \\
& \beta_{3}=\left(\frac{a_{1}}{r}-\frac{\gamma}{\nu}\right) a_{2}-\bar{\tau}_{2} \cdot \nabla a_{1}+\left(a_{2, r}-a_{1, z}\right) a_{1}+\frac{a_{1} a_{2}}{r}, \\
& \beta_{4}=-\left(\frac{a_{1}}{r}-\frac{\gamma}{\nu}\right) a_{1}-\bar{\tau}_{2} \cdot \nabla a_{2}+\left(a_{2, r}-a_{1, z}\right) a_{2}+\frac{a_{2}^{2}}{r} \\
& \beta_{5}=\bar{\tau}_{2} \cdot \nabla\left(\frac{a_{1}}{r}-\frac{\gamma}{\nu}\right)+\frac{1}{r}\left(\bar{n} \cdot \nabla a_{2}-\frac{a_{1} a_{2}}{r}\right) .
\end{aligned}
$$

This gives (3.8) and concludes the proof.

3.3. Energy type estimates for velocity and its angular derivative $h$. Repeating the proof of Lemma 3.3 from [zaj 5, Ch. 4] we have

Lemma 3.3. Let $\eta=\bar{e}_{0} \times \bar{x}, \bar{e}_{0}=(0,0,1), \bar{x}=\left(x_{1}, x_{2}, x_{3}\right), \eta=\bar{x}_{, \varphi}$, and let $\Omega$ have the axis of symmetry $\bar{e}_{0}$. Let $v$ be a solution to problem (1.1) and

$$
\left|\int_{\Omega} v_{0} \cdot \eta d x\right|<\infty, \quad\left|\int_{\Omega^{t}} f \cdot \eta d x d t^{\prime}\right|<\infty .
$$


Then

$$
\int_{\Omega} v \cdot \eta d x+\gamma \int_{S^{t}} v \cdot \eta d x d t^{\prime}=\int_{\Omega} v_{0} \cdot \eta d x+\int_{\Omega^{t}} f \cdot \eta d x d t^{\prime} .
$$

Proof. Multiplying $(1.1)_{1}$ by $\eta$, integrating the result over $\Omega^{t}$, using the boundary and initial conditions and the fact that $\nabla \eta$ is antisymmetric tensor we obtain (3.17). This ends the proof.

Now we examine the Korn inequality which is necessary to prove the estimate for weak solutions to problem (1.1). By the Korn inequality we mean an estimate such that the $H^{1}$ norm of a vector function is bounded in terms of the $L_{2}$ norm of its dilatation tensor.

To prove the Korn inequality we introduce

$$
E_{\Omega}(v)=\int_{\Omega}\left(v_{i, x_{j}}+v_{j, x_{i}}\right)^{2} d x,
$$

where the summation convention is assumed.

Lemma 3.4. Let $\left|\int_{\Omega} r v_{\varphi} d x\right|<\infty, E_{\Omega}(v)<\infty$. Then

$$
\|v\|_{1, \Omega}^{2} \leq c\left(E_{\Omega}(v)+\left|\int_{\Omega} r v_{\varphi} d x\right|^{2}\right) .
$$

Proof. Since $\eta=\bar{x}_{, \varphi}$ we have $r v_{\varphi}=v \cdot \eta \equiv v_{\eta}$. Let $\alpha_{*}=\int_{\Omega} v_{\eta} d x$. Then

$$
v=v^{\prime}+\frac{\alpha_{*}}{\int_{\Omega}|\eta|^{2} d x} \eta
$$

where

$$
v^{\prime}=v_{r} \bar{e}_{r}+\left(v_{\varphi}-\frac{\alpha_{*}}{\int_{\Omega}|\eta|^{2} d x} r\right) \bar{e}_{\varphi}+v_{z} \bar{e}_{z}, \quad \int_{\Omega} v^{\prime} \cdot \eta d x=0 .
$$

Since $E_{\Omega}(v)=2\left(|\nabla v|_{2, \Omega}^{2}-\int_{S} v_{i} v_{j} n_{i, x_{j}} d S\right)$ we have (see also [sos])

$$
|\nabla v|_{2, \Omega}^{2} \leq c\left(E_{\Omega}(v)+|v|_{2, S}^{2}\right) .
$$

By the trace theorem we obtain

$$
|\nabla v|_{2, \Omega}^{2} \leq c\left(E_{\Omega}(v)+|v|_{2, \Omega}^{2}\right) .
$$

Let $s_{1}, s_{2} \in S$ be two different points such that $a_{1}\left(s_{1}\right) a_{2}\left(s_{2}\right)-a_{1}\left(s_{2}\right) a_{2}\left(s_{1}\right) \neq 0$. By the Poincaré inequality

$$
\begin{aligned}
& \left|v_{r} a_{1}\left(s_{1}\right)+v_{z} a_{2}\left(s_{1}\right)\right|_{2, \Omega} \leq c\left|\nabla\left(v_{r} a_{1}\left(s_{1}\right)+v_{z} a_{2}\left(s_{1}\right)\right)\right|_{2, \Omega}, \\
& \left|v_{r} a_{1}\left(s_{2}\right)+v_{z} a_{2}\left(s_{2}\right)\right|_{2, \Omega} \leq c\left|\nabla\left(v_{r} a_{1}\left(s_{2}\right)+v_{z} a_{2}\left(s_{2}\right)\right)\right|_{2, \Omega} .
\end{aligned}
$$

Hence

$$
\left|v_{r}\right|_{2, \Omega}^{2}+\left|v_{z}\right|_{2, \Omega}^{2} \leq c\left(\left|\nabla v_{r}\right|_{2, \Omega}^{2}+\left|\nabla v_{z}\right|_{2, \Omega}^{2}\right)
$$

Repeating considerations from the proof of Lemma 4.2.4 from [zaj 5] we obtain

$$
|v|_{2, \Omega}^{2} \leq \delta\left|\nabla v^{\prime}\right|_{2, \Omega}^{2}+M(\delta) E_{\Omega}(v)+c\left|v_{\eta}\right|_{2, \Omega}^{2},
$$

where $\delta$ can be chosen sufficiently small. 
Employing (3.24) in (3.22) and assuming that $\delta$ is sufficiently small we get

$$
|\nabla v|_{2, \Omega}^{2} \leq c\left(E_{\Omega}(v)+\left|v_{\eta}\right|_{2, \Omega}^{2}\right) .
$$

Next

$$
\begin{aligned}
\left|v_{\varphi}\right|_{2, \Omega}^{2} & \leq c\left(\left|v_{r}\right|_{2, \Omega}^{2}+\left|v_{z}\right|_{2, \Omega}^{2}+\left|v^{\prime}\right|_{2, \Omega}^{2}+\left|\int_{\Omega} v_{\eta} d x\right|^{2}\right) \\
& \leq c\left(E_{\Omega}(v)+\left|\int_{\Omega} v_{\eta} d x\right|^{2}\right) .
\end{aligned}
$$

Collecting the above considerations implies (3.19). This ends the proof.

Lemma 3.5. Assume that $v(0) \in L_{2}(\Omega), f \in L_{2,1}\left(\Omega^{T}\right)$ and $\left|\int_{\Omega^{t}} v_{\eta} d x d t^{\prime}\right|<\infty$. Then

$$
|v(t)|_{2, \Omega} \leq|f|_{2,1, \Omega^{t}}+|v(0)|_{2, \Omega}, \quad t \leq T,
$$

and

$$
|v(t)|_{2, \Omega}^{2}+\nu \int_{0}^{t}\left\|v\left(t^{\prime}\right)\right\|_{1, \Omega}^{2} d t^{\prime} \leq c(1+t)\left(|f|_{2,1, \Omega^{t}}^{2}+|v(0)|_{2, \Omega}^{2}\right) .
$$

Proof. Multiplying (1.1) $)_{1}$ by $v$, integrating over $\Omega$ and applying the boundary conditions yields

$$
\frac{1}{2} \frac{d}{d t}|v|_{2, \Omega}^{2}+E_{\Omega}(v)+\gamma\left|v_{\tau}\right|_{2, S}^{2}=\int_{\Omega} f \cdot v d x
$$

Hence

$$
\frac{d}{d t}|v|_{2, \Omega} \leq|f|_{2, \Omega}
$$

Integrating (3.28) with respect to $t$ yields (3.25).

Making use of (3.19) in (3.27) implies

$$
\frac{1}{2} \frac{d}{d t}|v(t)|_{2, \Omega}^{2}+\nu\|v\|_{1, \Omega}^{2} \leq c|v|_{2, \Omega}|f|_{2, \Omega}+c|v|_{2, \Omega}^{2} .
$$

Integrating (3.29) with respect to time and using (3.25) gives (3.26). This ends the proof. Lemma 3.6. Assume that $h(0) \in L_{2}(\Omega), g \in L_{2}\left(\Omega^{T}\right)$ and $v \in L_{2}\left(0, T ; W_{3}^{1}(\Omega)\right)$. Then solutions of problem (1.4) satisfy

$$
|h(t)|_{2, \Omega}^{2}+\nu \int_{0}^{t}\left\|h\left(t^{\prime}\right)\right\|_{1, \Omega}^{2} d t^{\prime} \leq e^{c \int_{0}^{t}\left|\nabla v\left(t^{\prime}\right)\right|_{3, \Omega}^{2} d t^{\prime}}\left[|g|_{2, \Omega^{t}}^{2}+|h(0)|_{2, \Omega}^{2}\right]
$$

for all $t \leq T$.

Proof. Since $h_{\eta}=r v_{\varphi, \varphi}$ we have $\int_{\Omega} h_{\eta} d x=0$. Therefore repeating the proof of Lemma 3.4 implies

$$
\|h\|_{1, \Omega}^{2} \leq c E_{\Omega}(h)
$$

Multiplying (1.4) 1 by $h$, integrating over $\Omega$ and using (3.31) yields

$$
\frac{d}{d t}|h|_{2, \Omega}^{2}+\nu\|h\|_{1, \Omega}^{2} \leq c\left(|\nabla v|_{3, \Omega}^{2}|h|_{2, \Omega}^{2}+|g|_{2, \Omega}^{2}\right) \text {. }
$$

Integrating (3.32) with respect to time gives (3.30). This ends the proof. 
3.4. Reformulation of the problem for the azimuthal component of the vorticity $\chi$. To prove global existence of solutions to problem (1.1) we need very delicate estimates for the vorticity vector. In this paper, in contrast to [zaj 5], problems for $\alpha_{1}$ and $\alpha_{3}$ are coupled by boundary conditions (see (1.7)). Similarly to [zaj 5] the most important is an energy type estimate for $\alpha_{2}$ which is distinguished by denoting it by $\chi$ (see $\left.(1.6)_{2}\right)$. However, unlike [zaj 5], we have a nonhomogeneous boundary condition for $\chi$ (see $\left.(1.8)_{2}\right)$. Hence to apply the energy method to problem (1.8) we introduce a function $\beta$ such that

$$
\begin{array}{ll}
\beta_{t}-\nu \Delta \beta=0 & \text { in } \Omega^{T}, \\
\left.\beta\right|_{S}=2(k-\gamma /(2 \nu)) v \cdot \bar{\tau}_{2} \equiv \chi_{1} & \text { on } S^{T}, \\
\left.\beta\right|_{t=0}=0 & \text { in } \Omega .
\end{array}
$$

Let us introduce the new function

$$
\chi^{\prime}=\chi-\beta
$$

In view of (3.33) and (1.8), $\chi^{\prime}$ is a solution to the problem

$$
\begin{aligned}
& \chi_{, t}^{\prime}+ v \cdot \nabla \chi^{\prime}-\frac{w_{, \varphi}}{r} \chi^{\prime}-\frac{v_{r}}{r} \chi^{\prime}+\frac{\nu}{r^{2}} \chi^{\prime}-\nu \Delta \chi^{\prime} \\
&= F_{2}-v \cdot \nabla \beta+\frac{w_{, \varphi}}{r} \beta+\frac{v_{r}}{r} \beta+\frac{2 \nu}{r^{2}}\left(\frac{1}{r} h_{z, \varphi}-h_{\varphi, z}\right) \\
& \quad-\frac{1}{r}\left(w_{, z} h_{r}-w_{, r} h_{z}+\frac{w}{r} h_{z}\right)+\frac{2}{r} w v_{\varphi, z}, \\
&\left.\chi^{\prime}\right|_{S}= 0, \\
&\left.\chi^{\prime}\right|_{t=0}=\chi(0) .
\end{aligned}
$$

It is convenient to express the last two terms on the l.h.s. of $(3.35)_{1}$ in the form

$$
-\nu \Delta \chi^{\prime}+\frac{\nu}{r^{2}} \chi^{\prime}=-\nu\left[\left(r\left(\frac{\chi^{\prime}}{r}\right)_{, r}\right)_{, r}+\frac{1}{r^{2}} \chi_{, \varphi \varphi}^{\prime}+\chi_{, z z}^{\prime}+2\left(\frac{\chi^{\prime}}{r}\right)_{, r}\right] .
$$




\section{Estimates for vorticity and azimuthal derivatives of velocity}

In this section we find energy estimates and estimates in $W_{2,1-\mu}^{2,1}\left(\Omega^{T}\right), \mu \in(1 / 2,1)$, for the vorticity vector.

4.1. Energy estimate for $\chi$. Now we obtain an estimate for $\chi$.

Lemma 4.1. Assume that $k-\gamma /(2 \nu) \in V_{4,-\delta / 2}^{3 / 2}(S), \varepsilon, \varepsilon_{1}$ are small positive numbers, $\delta \geq 1$, $v_{\varphi, z} \in L_{2}\left(0, t ; L_{4,-3 / 4-\varepsilon}(\Omega)\right)$. Assume that $h \in H_{-1}^{2,1}\left(\Omega^{t}\right), v \in A_{1-\mu}\left(\Omega^{t}\right), \mu \in(1 / 2,1)$, $w \in L_{\infty}\left(0, t ; H_{0}^{1}(\Omega)\right), F_{\varphi} \in L_{2,-1}\left(\Omega^{t}\right), \chi(0) \in L_{2,-1}(\Omega), t \in[0, T]$. Then any solution of (3.35) satisfies

$$
\begin{aligned}
|\chi|_{2,-1, \Omega}^{2}+ & \nu\left|\nabla \frac{\chi}{r}\right|_{2, \Omega^{t}}^{2} \leq c\left(\|h\|_{2,-1, \Omega^{t}}^{2}+\left|v_{r}\right|_{10 / 3, \Omega^{t}}^{2}+1\right)\|k-\gamma /(2 \nu)\|_{3 / 2,4,-\delta / 2, S}^{2} \\
& \cdot\left(\varepsilon_{1}\|v\|_{A_{1-\mu}\left(\Omega^{t}\right)}+c\left(\varepsilon^{-3 / 4}\right)|v|_{2, \Omega^{t}}\right) \\
& +c\left[\sup _{t}\left|h_{\varphi}\right|_{2,-1, \Omega}|\chi|_{2,-1, \Omega^{t}}^{2}+\left(1+\sup _{t}\|w\|_{1,0, \Omega}^{2}\right)\|h\|_{2,-1, \Omega^{t}}^{2}\right. \\
& \left.+\sup _{t}\|w\|_{1,0, \Omega}^{2} \int_{0}^{t}\left|v_{\varphi, z}\left(t^{\prime}\right)\right|_{4,-3 / 4-\varepsilon, \Omega}^{2} d t^{\prime}+\left|F_{\varphi}\right|_{2,-1, \Omega^{t}}^{2}\right]+\left|\chi^{\prime}(0)\right|_{2,-1, \Omega}^{2} .
\end{aligned}
$$

Proof. To obtain the estimate we write problem (3.35) in the form

$$
\begin{aligned}
\chi_{, t}^{\prime}+ & v_{r} \chi_{, r}^{\prime}+\frac{v_{\varphi}}{r} \chi_{, \varphi}^{\prime}+v_{z} \chi_{, z}^{\prime}+\left(v_{r, r}+v_{z, z}\right) \chi^{\prime} \\
& -\nu\left(\chi_{, r r}^{\prime}+\frac{1}{r^{2}} \chi_{, \varphi \varphi}^{\prime}+\chi_{, z z}^{\prime}+\frac{1}{r} \chi_{, r}^{\prime}-\frac{1}{r^{2}} \chi^{\prime}\right) \\
= & -\left[v_{r} \beta_{, r}+\frac{v_{\varphi}}{r} \beta_{, \varphi}+v_{z} \beta_{, z}+\left(v_{r, r}+v_{z, z}\right) \beta+\nu \frac{\beta}{r^{2}}\right] \\
& -\frac{2 \nu}{r^{2}}\left(h_{\varphi, z}-\frac{1}{r} h_{z, \varphi}\right)-\frac{1}{r}\left(w_{, z} h_{r}-w_{, r} h_{z}+\frac{w}{r} h_{z}\right)+\frac{2 w v_{\varphi, z}}{r}+F_{\varphi}, \\
\left.\chi^{\prime}\right|_{S}= & 0 \\
\left.\chi^{\prime}\right|_{t=0} & =\chi^{\prime}(0) .
\end{aligned}
$$

Multiplying (4.2) $)_{1}$ by $\chi^{\prime} / r^{2}$, integrating over $\Omega_{\varepsilon}=\{x \in \Omega: x \notin \Omega \cap\{r \leq \varepsilon, z \in(-a, a)$, $\varphi \in[0,2 \pi]\}, \varepsilon>0\}$, and using the boundary condition $(4.2)_{2}$ we obtain 


$$
\begin{aligned}
\frac{1}{2} & \frac{d}{d t}\left|\chi^{\prime}\right|_{2,-1, \Omega}^{2}+\int_{\Omega_{\varepsilon}} v \cdot \nabla \chi^{\prime} \frac{\chi^{\prime}}{r^{2}} d x+\int_{\Omega_{\varepsilon}}\left(v_{r, r}+v_{z, z}\right) \frac{\chi^{\prime 2}}{r^{2}} d x \\
& -\nu \int_{\Omega_{\varepsilon}}\left[\left(r\left(\frac{\chi^{\prime}}{r}\right)_{, r}\right)_{, r}+\frac{1}{r^{2}} \chi_{, \varphi \varphi}^{\prime}+\chi_{, z z}^{\prime}+2\left(\frac{\chi^{\prime}}{r}\right)_{, r}\right] \frac{\chi^{\prime}}{r^{2}} d x \\
& =-\int_{\Omega_{\varepsilon}}\left[v \cdot \nabla \beta+\left(v_{r, r}+v_{z, z}\right) \beta+\nu \frac{\beta}{r^{2}}\right] \frac{\chi^{\prime}}{r^{2}} d x-2 \nu \int_{\Omega_{\varepsilon}} \frac{1}{r^{2}}\left(h_{\varphi, z}-\frac{1}{r} h_{z, \varphi}\right) \frac{\chi^{\prime}}{r^{2}} d x \\
& -\int_{\Omega_{\varepsilon}} \frac{1}{r}\left(w_{, z} h_{r}-w_{, r} h_{z}+\frac{w}{r} h_{z}\right) \frac{\chi^{\prime}}{r^{2}} d x+2 \int_{\Omega_{\varepsilon}} \frac{w v_{\varphi, z}}{r} \frac{\chi^{\prime}}{r^{2}} d x+\int_{\Omega_{\varepsilon}} F_{\varphi} \frac{\chi^{\prime}}{r^{2}} d x .
\end{aligned}
$$

Now we examine the particular terms in (4.3). The sum of the second and third terms on the l.h.s. of (4.3) equals

$$
\begin{aligned}
\frac{1}{2} \int_{\Omega_{\varepsilon}} \frac{1}{r^{2}} v \cdot \nabla \chi^{\prime 2} d x+\int_{\Omega_{\varepsilon}}\left(v_{r, r}+v_{z, z}\right) \frac{\chi^{\prime 2}}{r^{2}} d x=\frac{1}{2} \int_{\Omega_{\varepsilon}} \operatorname{div}\left(\frac{v}{r^{2}} \chi^{\prime 2}\right) d x \\
\quad-\frac{1}{2} \int_{\Omega_{\varepsilon}} \chi^{\prime 2} v \cdot \nabla\left(\frac{1}{r^{2}}\right) d x+\int_{\Omega_{\varepsilon}}\left(v_{r, r}+v_{z, z}\right) \frac{\chi^{\prime 2}}{r^{2}} d x=\frac{1}{2} \int_{\partial \Omega_{\varepsilon}} v \cdot \bar{n} \frac{\chi^{\prime 2}}{r^{2}} d \partial \Omega_{\varepsilon} \\
+\int_{\Omega_{\varepsilon}}\left(v_{r, r}+v_{z, z}+\frac{v_{r}}{r}\right) \frac{\chi^{\prime 2}}{r^{2}} d x=\frac{1}{2} \int_{\partial \Omega_{\varepsilon}} v \cdot \bar{n} \frac{\chi^{\prime 2}}{r^{2}} d \partial \Omega_{\varepsilon}-\int_{\Omega_{\varepsilon}} \frac{v_{\varphi, \varphi}}{r} \frac{\chi^{\prime 2}}{r^{2}} d x
\end{aligned}
$$

where (2.41) was used.

In view of the above considerations we see that to examine (4.3) we have to add boundary conditions on the part of the boundary of $\Omega_{\varepsilon}$ determined by $r=\varepsilon, z \in(-a, a)$, $\varphi \in[0,2 \pi]$. The first term on the l.h.s. of (4.3) implies an estimate for $\left|\chi^{\prime}\right|_{2,-1, \Omega}$. Since the norm implies vanishing of $\chi^{\prime}$ at $r=0$ we can assume that $\left.\chi\right|_{r=\varepsilon}=0$.

Then the sum of the second and third terms on the l.h.s. of (4.3) equals

$$
-\int_{\Omega_{\varepsilon}} \frac{v_{\varphi, \varphi}}{r} \frac{\chi^{\prime 2}}{r^{2}} d x
$$

The last term on the l.h.s. of (4.3) equals

$$
-\nu \int_{0}^{2 \pi} d \varphi \int_{\Omega_{\varepsilon}(\varphi)}\left[\left[r\left(\frac{\chi^{\prime}}{r}\right)_{, r}\right]_{, r}+2\left(\frac{\chi^{\prime}}{r}\right)_{, r}\right] \frac{\chi}{r} d r d z-\nu \int_{\Omega_{\varepsilon}}\left(\frac{1}{r^{2}} \chi_{, \varphi \varphi}^{\prime}+\chi_{, z z}^{\prime}\right) \frac{\chi^{\prime}}{r^{2}} d x \equiv I_{1},
$$

where $\Omega_{\varepsilon}\left(\varphi_{0}\right)=\left\{x \in \Omega_{\varepsilon}: \varphi=\varphi_{0}\right\}$. Since $\Omega_{\varepsilon}(\varphi)$ is a domain in $\mathbb{R}^{2}$ with measure equal to $\int_{\Omega_{\varepsilon}(\varphi)} d r d z$, the first term in $I_{1}$ takes the form

$$
-\nu \int_{0}^{2 \pi} d \varphi \int_{\Omega_{\varepsilon}(\varphi)}\left[r\left(\frac{\chi^{\prime}}{r}\right)_{, r} \frac{\chi^{\prime}}{r}+\left(\frac{\chi^{\prime}}{r}\right)^{2}\right]_{, r} d r d z+\nu \int_{\Omega_{\varepsilon}}\left(\frac{\chi^{\prime}}{r}\right)_{, r}^{2} d x
$$


where the first term equals

$$
-\left.\nu \int_{0}^{2 \pi} d \varphi \int_{-a}^{a} d z\left[r\left(\frac{\chi^{\prime}}{r}\right)_{, r} \frac{\chi^{\prime}}{r}+\left(\frac{\chi^{\prime}}{r}\right)^{2}\right]\right|_{r=\varepsilon} ^{r=\psi(z)}=-\left.\nu \int_{0}^{2 \pi} d \varphi \int_{-a}^{a} d z \frac{\chi_{, r}^{\prime} \chi^{\prime}}{r}\right|_{r=\varepsilon} ^{r=\psi(z)} \equiv I_{2},
$$

where $r=\psi(z)$ describes the boundary of $\Omega_{\varepsilon}(\varphi), \varphi \in(0,2 \pi)$.

Since $\left.\chi^{\prime}\right|_{S}=0$ and $\left.\chi^{\prime}\right|_{r=\varepsilon}=0$ is assumed as an artificial boundary condition we examine the expression

$$
I_{2}=\left.\nu \int_{0}^{2 \pi} d \varphi \int_{-a}^{a} d z \frac{\chi_{, r}^{\prime} \chi^{\prime}}{r}\right|_{r=\varepsilon}
$$

for $\varepsilon$ small.

Expecting that $\chi^{\prime}$ will belong to $L_{\infty}\left(0, T ; H_{-1}^{1}(\Omega)\right)$ we will have $\left.\chi^{\prime}\right|_{r=0}=0$. Without assuming that $\left.\chi^{\prime}\right|_{r=\varepsilon}=0$ we see in a neighbourhood of $r=0$ that if $\chi^{\prime}>0$ then $\chi_{, r}^{\prime}>0$ and if $\chi^{\prime}<0$ then $\chi_{, r}^{\prime}<0$. Therefore $I_{2}>0$ so we do not need any estimate for it because it remains on the l.h.s. of the expected inequality. Hence to examine $I_{1}$ we do not need to assume that $\left.\chi^{\prime}\right|_{r=\varepsilon}=0$.

Finally, the second term in $I_{1}$ takes the form

$$
\nu \int_{\Omega_{\varepsilon}}\left(\frac{\chi_{, \varphi}^{\prime 2}}{r^{4}}+\frac{\chi_{, z}^{\prime 2}}{r^{2}}\right) d x
$$

The first term on the r.h.s. of (4.3) equals

$$
\begin{array}{r}
-\int_{0}^{2 \pi} d \varphi \int_{\Omega_{\varepsilon}(\varphi)}\left(v_{r} \beta_{, r}+\frac{v_{\varphi}}{r} \beta_{, \varphi}+v_{z} \beta_{, z}\right) \frac{\chi^{\prime}}{r} d r d z-\int_{\Omega_{\varepsilon}}\left(v_{r, r}+v_{z, z}\right) \beta \frac{\chi^{\prime}}{r^{2}} d x \\
-\int_{\Omega_{\varepsilon}} \frac{\beta}{r^{2}} \frac{\chi^{\prime}}{r^{2}} d x \equiv I_{3},
\end{array}
$$

where the first term takes the form

$$
\int_{\Omega_{\varepsilon}}\left(v_{r, r}+\frac{v_{\varphi, \varphi}}{r}+v_{z, z}\right) \frac{\beta \chi^{\prime}}{r^{2}} d x+\int_{\Omega_{\varepsilon}} v \cdot \nabla\left(\frac{\chi^{\prime}}{r}\right) \frac{\beta}{r} d x
$$

Hence

$$
I_{3}=\int_{\Omega_{\varepsilon}} \frac{v_{\varphi, \varphi}}{r} \frac{\beta \chi^{\prime}}{r^{2}} d x+\int_{\Omega_{\varepsilon}} v \cdot \nabla\left(\frac{\chi^{\prime}}{r}\right) \frac{\beta}{r} d x-\nu \int_{\Omega_{\varepsilon}} \frac{\beta}{r^{2}} \frac{\chi^{\prime}}{r^{2}} d x .
$$

Estimating, we obtain

$$
\left|I_{3}\right| \leq \varepsilon_{1} \int_{\Omega_{\varepsilon}}\left(\left|\nabla \frac{\chi^{\prime}}{r}\right|^{2}+\frac{\chi^{\prime 2}}{r^{4-\delta}}\right) d x+c\left(\frac{1}{\varepsilon_{1}}\right) \int_{\Omega_{\varepsilon}}\left(\frac{v_{\varphi, \varphi}^{2}}{r^{2+\delta}} \beta^{2}+\frac{v^{2} \beta^{2}}{r^{2}}+\frac{\beta^{2}}{r^{4+\delta}}\right) d x
$$

for any $\varepsilon_{1}>0, \delta>0$.

The second term on the r.h.s. of (4.3) equals

$$
2 \nu \int_{\Omega_{\varepsilon}}\left[\frac{1}{r^{2}} h_{\varphi} \frac{\chi_{, z}^{\prime}}{r^{2}}-\frac{1}{r^{3}} h_{z} \frac{\chi_{, \varphi}^{\prime}}{r^{2}}\right] d x \equiv I_{4},
$$


so, for all $\varepsilon_{2}>0$,

$$
\left|I_{4}\right| \leq \varepsilon_{2} \int_{\Omega_{\varepsilon}}\left[\left(\frac{\chi^{\prime}}{r}\right)_{, z}^{2}+\chi_{, \varphi}^{\prime 2}\right] d x+c\left(1 / \varepsilon_{2}\right) \int_{\Omega_{\varepsilon}} \frac{1}{r^{6}}\left(h_{\varphi}^{2}+h_{z}^{2}\right) d x,
$$

where the last term is bounded by $c\|h\|_{2,-1, \Omega_{\varepsilon}}^{2}$.

By the Hölder and Young inequalities we estimate the third term on the r.h.s. of (4.3) by

$$
\varepsilon_{3}\left|\frac{\chi^{\prime}}{r}\right|_{6, \Omega_{\varepsilon}}^{2}+c\left(1 / \varepsilon_{3}\right)\|w\|_{1,0, \Omega}^{2}|h|_{3,-2, \Omega_{\varepsilon}}^{2}
$$

for any $\varepsilon_{3}>0$, where we use the imbedding

$$
|h|_{3,-2, \Omega} \leq c\|h\|_{1,-2, \Omega} \leq c\|h\|_{2,-1, \Omega} .
$$

Finally, the fourth term on the r.h.s. of (4.3) is bounded by

$$
\begin{aligned}
& \varepsilon_{4}\left|\frac{\chi^{\prime}}{r^{2-\varepsilon^{\prime}}}\right|_{2, \Omega_{\varepsilon}}^{2}+c\left(1 / \varepsilon_{4}\right)|w|_{4,-1 / 4, \Omega_{\varepsilon}}^{2}\left|v_{\varphi, z}\right|_{4,-3 / 4-\varepsilon^{\prime}, \Omega_{\varepsilon}}^{2} \\
& \leq \varepsilon_{4}\left|\frac{\chi^{\prime}}{r^{2-\varepsilon^{\prime}}}\right|_{2, \Omega_{\varepsilon}}^{2}+c\left(1 / \varepsilon_{4}\right)\|w\|_{1,0, \Omega_{\varepsilon}}^{2}\left|v_{\varphi, z}\right|_{4,-3 / 4-\varepsilon^{\prime}, \Omega_{\varepsilon}}^{2}
\end{aligned}
$$

for any $\varepsilon_{4}, \varepsilon^{\prime}>0$. Summarizing the above considerations we obtain

$$
\begin{aligned}
\frac{d}{d t}\left|\chi^{\prime}\right|_{2,-1, \Omega_{\varepsilon}}^{2} & +\nu \int_{\Omega_{\varepsilon}}\left|\nabla \frac{\chi^{\prime}}{r}\right|^{2} d x \leq c\left|h_{\varphi}\right|_{2,-1, \Omega_{\varepsilon}}\left|\chi^{\prime}\right|_{4,-1, \Omega_{\varepsilon}}^{2} \\
& +c\|h\|_{2,-1, \Omega_{\varepsilon}}^{2}+c\|w\|_{1,0, \Omega_{\varepsilon}}^{2}\|h\|_{2,-1, \Omega_{\varepsilon}}^{2}+c\|w\|_{1,0, \Omega_{\varepsilon}}^{2}\left|v_{\varphi, z}\right|_{4,-3 / 4-\varepsilon^{\prime}, \Omega_{\varepsilon}}^{2} \\
& +c\left|F_{\varphi}\right|_{2,-1, \Omega_{\varepsilon}}^{2}+c \int_{\Omega_{\varepsilon}}\left(\frac{h_{\varphi}^{2}}{r^{2+\delta}} \beta^{2}+\frac{v^{2} \beta^{2}}{r^{2}}+\frac{\beta^{2}}{r^{4+\delta}}\right) d x
\end{aligned}
$$

for any $\delta, \varepsilon, \varepsilon^{\prime}>0$.

Integrating (4.4) with respect to time and passing with $\varepsilon$ to 0 we obtain

$$
\begin{aligned}
& \left|\chi^{\prime}\right|_{2,-1, \Omega}^{2}+\nu\left|\nabla \frac{\chi^{\prime}}{r}\right|_{2, \Omega^{t}}^{2} \leq c\left[\sup _{t}\left|h_{\varphi}\right|_{2,-1, \Omega} \int_{0}^{t}\left\|\frac{\chi^{\prime}}{r}\right\|_{1, \Omega}^{2} d t\right. \\
& +\|h\|_{2,-1, \Omega^{t}}^{2}+\sup _{t}\|w\|_{1,0, \Omega}^{2}\|h\|_{2,-1, \Omega^{t}}^{2}+\sup _{t}\|w\|_{1,0, \Omega}^{2} \int_{0}^{t}\left|v_{\varphi, z}\left(t^{\prime}\right)\right|_{4,-3 / 4-\varepsilon^{\prime}, \Omega}^{2} d t^{\prime} \\
& \left.\quad+\left|F_{\varphi}\right|_{2,-1, \Omega^{t}}^{2}+\int_{\Omega^{t}}\left(\frac{h_{\varphi}^{2}}{r^{2+\delta}} \beta^{2}+\frac{v^{2} \beta^{2}}{r^{2}}+\frac{\beta^{2}}{r^{4+\delta}}\right) d x\right]+\left|\chi^{\prime}(0)\right|_{2,-1, \Omega}^{2} .
\end{aligned}
$$

Let us denote the last integral by $I$. Then we have

$$
\begin{aligned}
I & \leq c\|h\|_{2,-1, \Omega^{t}}^{2}|\beta|_{2, \Omega^{t}}^{2}+|v|_{10 / 3, \Omega^{t}}^{2}|\beta|_{5,-1, \Omega^{t}}^{2}+|\beta|_{2,-(2+\delta / 2), \Omega^{t}}^{2} \\
& \leq c\left(\|h\|_{2,-1, \Omega^{t}}^{2}+|v|_{10 / 3, \Omega^{t}}^{2}+1\right)\|\beta\|_{2,-\delta / 2, \Omega^{t}}^{2}
\end{aligned}
$$

for all $\delta \geq 1$ and 


$$
\begin{aligned}
\|\beta\|_{2,-\delta / 2, \Omega^{t} \leq} \leq & \left\|(k-\gamma /(2 \nu)) v \cdot \bar{\tau}_{2}\right\|_{3 / 2,-\delta / 2, S^{t}} \\
\leq & c\left(\int_{S}\left|\partial_{s}^{3 / 2}(k-\gamma /(2 \nu))\right|^{4} r^{-2 \delta} d S\right)^{1 / 4}\left(\int_{0}^{t} d t^{\prime}\left|v\left(t^{\prime}\right)\right|_{4, S}^{2}\right)^{1 / 2} \\
& +c\left(\int_{S}|k-\gamma /(2 \nu)|^{4} r^{-2 \delta} d S\right)^{1 / 4} \cdot \\
& \cdot\left(\int_{0}^{t} d t^{\prime}\left(\left|\partial_{s}^{3 / 2}\left(v \cdot \bar{\tau}_{2}\right)\right|_{4, S}^{2}+\left|\partial_{t}^{3 / 4}\left(v \cdot \bar{\tau}_{2}\right)\right|_{4, S}^{2}\right)\right)^{1 / 2} \\
& +c\left|(k-\gamma /(2 \nu)) v \cdot \bar{\tau}_{2}\right|_{2,-2-\delta / 2, S^{t}} \leq c|| k-\gamma /(2 \nu) \|_{3 / 2,4,-\delta / 2, S} \\
& \cdot\left(\int_{0}^{t} d t^{\prime}\left[\varepsilon\left(\left|v_{, x x x}\right|_{2,1-\mu, \Omega}^{2}+\left|v_{, t x}\right|_{2,1-\mu, \Omega}^{2}\right)+c(1 / \varepsilon)|v|_{2, \Omega}^{2}\right]\right)^{1 / 2} \\
\leq & c\|k-\gamma /(2 \nu)\|_{3 / 2,4,-\delta / 2, S}\left(\varepsilon\|v\|_{A_{1-\mu}\left(\Omega^{t}\right)}+c(1 / \varepsilon)|v|_{2, \Omega^{t}}\right)
\end{aligned}
$$

for all $\varepsilon>0$, where $c(1 / \varepsilon)=c \varepsilon^{-3 / 4}$.

Employing the above considerations in (4.5) yields (4.1). This concludes the proof.

\subsection{Estimate of $h$ in weighted Sobolev spaces}

Lemma 4.2. Assume that $h_{0} \in H_{-1}^{1}(\Omega), g \in L_{2,-1}\left(\Omega^{T}\right), v_{, x} \in W_{2,1-\mu}^{2,1}\left(\Omega^{T}\right), \mu>1 / 2$. Then for solutions of (1.4) we have

$$
\begin{aligned}
\|h\|_{2,-1, \Omega^{t}}+\left(\int_{0}^{t} \|\right. & \left.\nabla q\left(t^{\prime}\right) \|_{0,-1, \Omega}^{2} d t^{\prime}\right)^{1 / 2} \\
\leq & \varphi\left(\left\|v_{, x}\right\|_{2,2,1-\mu, \Omega^{t}}, t^{1 / 2}\left\|v_{, x}\right\|_{2,2,1-\mu, \Omega^{t}}\right) \\
& \cdot\left[|h(0)|_{2, \Omega}+\int_{0}^{t}\left|g\left(t^{\prime}\right)\right|_{2, \Omega} d t^{\prime}\right]+c\left(\|g\|_{0,-1, \Omega^{t}}+\|h(0)\|_{1,-1, \Omega}\right)
\end{aligned}
$$

for all $t \leq T$, where $\varphi$ is an increasing positive function.

Proof. In view of [zaj 4] solutions of (1.4) satisfy

$$
\|h\|_{2,-1, \Omega^{t}}+\left(\int_{0}^{t}\|\nabla q\|_{0,-1, \Omega}^{2} d t^{\prime}\right)^{1 / 2} \leq c\left(\|G\|_{0,-1, \Omega^{t}}+\|h(0)\|_{1,-1, \Omega}\right)
$$

for all $t \leq T$. Now we estimate the separate terms in $G$. First we consider

$$
\begin{aligned}
|v \cdot \nabla h|_{2,-1, \Omega^{t}} & \leq|v|_{2 p_{1},-\mu, \Omega^{t}}|\nabla h|_{2 p_{2}, \mu-1, \Omega^{t}} \\
& \leq c\left|v_{, x}\right|_{2 p_{1}, 1-\mu, \Omega^{t}}|\nabla h|_{2 p_{2}, \mu-1, \Omega^{t}} \equiv I_{1}
\end{aligned}
$$

whenever $1 / p_{1}+1 / p_{2}=1$, where the Hardy inequality (see Lemma 2.10) was used.

In view of [zaj 3] we have, for all $\varepsilon>0$,

$$
I_{1} \leq \varepsilon\|h\|_{2, \mu-1, \Omega^{t}}+c(1 / \varepsilon) \varphi\left(\left\|v_{, x}\right\|_{2,1-\mu, \Omega^{t}}\right)|h|_{2, \mu-1, \Omega^{t}} \equiv I,
$$

where the imbeddings $D_{x} W_{2, \mu-1}^{2,1}\left(\Omega^{T}\right) \subset L_{2 p_{2}, \mu-1}\left(\Omega^{T}\right)$ and $W_{2,1-\mu}^{2,1}\left(\Omega^{T}\right) \subset L_{2 p_{1}, 1-\mu}\left(\Omega^{T}\right)$, which hold together, were used. 
Next we have

$$
|h \cdot \nabla v|_{2,-1, \Omega^{t}} \leq\left|v_{, x}\right|_{2 p_{1},-\mu, \Omega^{t}}|h|_{2 p_{2}, \mu-1, \Omega^{t}} \leq c\left|v_{, x x}\right|_{2 p_{1}, 1-\mu, \Omega^{t}}|h|_{2 p_{2}, \mu-1, \Omega^{t}} \equiv I_{2}
$$

whenever $1 / p_{1}+1 / p_{2}=1$; in the second inequality the Hardy inequality was used.

By the imbeddings $D_{x}^{2} W_{2,1-\mu}^{2,1}\left(\Omega^{T}\right) \subset L_{2 p_{1}, 1-\mu}\left(\Omega^{T}\right), W_{2, \mu-1}^{2,1}\left(\Omega^{T}\right) \subset L_{2 p_{2}, \mu-1}\left(\Omega^{T}\right)$, which hold together, we obtain $I_{2} \leq I$.

Using the above estimates in (4.9) yields

$$
\begin{aligned}
\|h\|_{2,-1, \Omega^{t}}+\left(\int_{0}^{t}\left\|\nabla q\left(t^{\prime}\right)\right\|_{0,-1, \Omega}^{2} d t^{\prime}\right)^{1 / 2} \leq & \varphi\left(\left\|v_{, x}\right\|_{2,2,1-\mu, \Omega^{t}}\right)|h|_{2, \mu-1, \Omega^{t}} \\
& +c\left(\|g\|_{0,-1, \Omega^{t}}+\|h(0)\|_{1,-1, \Omega}\right) .
\end{aligned}
$$

By [zaj 3] for $\mu>1 / 2$ we have

$$
|\nabla v|_{\infty, \Omega} \leq c\|\nabla v\|_{2,2,1-\mu, \Omega}
$$

so

$$
\int_{0}^{t}\left|\nabla v\left(t^{\prime}\right)\right|_{\infty,} d t^{\prime} \leq c t^{1 / 2}\left\|v_{, x}\right\|_{2,2,1-\mu, \Omega^{t}} .
$$

Next from (3.30) and by the Hardy inequality (see Lemma 2.8) we have

$$
\int_{0}^{t}\left|h\left(t^{\prime}\right)\right|_{2, \delta-1}^{2} d t^{\prime} \leq c \varphi\left(t^{1 / 2}\left\|v_{, x}\right\|_{2,2,1-\mu, \Omega^{t}}\right)\left[|h(0)|_{2, \Omega}^{2}+|g|_{2, \Omega^{t}}^{2}\right],
$$

where $\varphi$ is an increasing positive function and $\delta>0$.

Choosing $\delta=\mu$ in (4.11), we obtain from (4.10) and (4.11) the inequality (4.8). This concludes the proof.

4.3. Estimates of vorticity in weighted Sobolev spaces. Now we examine problem (1.7). Let $\alpha^{\prime}=\left(\alpha_{1}, \alpha_{3}\right)$.

LEMma 4.3. Assume that $v \in A_{1-\mu}\left(\Omega^{t}\right), \alpha_{2} / r \in L_{10 / 3}\left(\Omega^{t}\right), h \in H_{1-\mu}^{2,1}\left(\Omega^{t}\right), w \in H_{1-\mu}^{2,1}\left(\Omega^{t}\right)$, $F^{\prime} \in L_{2,1-\mu}\left(\Omega^{t}\right), \alpha^{\prime}(0) \in W_{2,1-\mu}^{1}(\Omega), \mu \geq 1 / 2, a_{1} \in C^{2}$ and $\left|\nabla^{2}\left(a_{1} / r\right)\right| \leq c r^{-2}$, $\left|\nabla\left(a_{1} / r\right)\right| \leq c r^{-1},\left|a_{1} / r\right| \leq c, \beta_{i} \in C^{1}, i=1, \ldots, 5$, and $\left|\nabla \beta_{j}\right| \leq c r^{-3},\left|\beta_{j}\right| \leq c r^{-2}, j=$ $1,2,\left|\nabla \beta_{k}\right| \leq c r^{-1},\left|\beta_{k}\right| \leq c, k=3,4,\left|\nabla \beta_{5}\right| \leq c r^{-2},\left|\beta_{5}\right| \leq c r^{-1}, \alpha^{\prime} \in L_{\infty}\left(0, t ; L_{2,1-\mu}(\Omega)\right)$ $\cap L_{2,-(1+\mu)}\left(\Omega^{t}\right), t \leq T$. Then

$$
\begin{aligned}
\left\|\alpha^{\prime}\right\|_{2,2,1-\mu, \Omega^{t}} \leq & \sup _{t}\left|\alpha^{\prime}\right|_{2,1-\mu, \Omega} \varphi\left(\|v\|_{A_{1-\mu}\left(\Omega^{t}\right)}\right)+c\left|\alpha_{2} / r\right|_{10 / 3, \Omega^{t}}\|h\|_{2,-\mu, \Omega^{t}} \\
& +c\left(\|h\|_{2,-\mu, \Omega^{t}}+\|w\|_{2,1-\mu, \Omega^{t}}\right)+c\left|\alpha_{1}\right|_{2,-(1+\mu), \Omega^{t}} \\
& +c\left|F^{\prime}\right|_{2,1-\mu, \Omega^{t}}+c\left\|\alpha^{\prime}(0)\right\|_{1,2,1-\mu, \Omega},
\end{aligned}
$$

where $\varphi$ is an increasing positive function.

Proof. Applying [zaj 1] to problem (1.7) we obtain

$$
\begin{aligned}
\left\|\alpha^{\prime}\right\|_{2,2,1-\mu, \Omega^{t}} \leq & c\left(\left|v \cdot \nabla \alpha_{1}\right|_{2,1-\mu, \Omega^{t}}\right. \\
& +\left|v \cdot \nabla \alpha_{3}\right|_{2,1-\mu, \Omega^{t}}+\left|\alpha_{1} v_{r, r}+\alpha_{3} v_{r, z}\right|_{2,1-\mu, \Omega^{t}}
\end{aligned}
$$




$$
\begin{aligned}
& +\left|\alpha_{1} v_{z, r}+\alpha_{3} v_{z, z}\right|_{2,1-\mu, \Omega^{t}}+\left|\frac{\alpha_{2}}{r} h_{r}\right|_{2,1-\mu, \Omega^{t}}+\left|\frac{1}{r^{2}}\left(h_{r, z}-h_{z, r}\right)\right|_{2,1-\mu, \Omega^{t}} \\
& +\left|\frac{\alpha_{1}}{r^{2}}\right|_{2,1-\mu, \Omega^{t}}+\left|F^{\prime}\right|_{2,1-\mu, \Omega^{t}}+\left\|\frac{a_{1}}{r} w \mid\right\|_{3 / 2,2,1-\mu, S^{t}} \\
& \left.+\left\|\beta_{1} h_{r}+\beta_{2} h_{z}+\beta_{3} w_{, r}+\beta_{4} w_{, z}+\beta_{5} w\right\|_{1 / 2,2,1-\mu, S^{t}}+\left\|\alpha^{\prime}(0)\right\|_{1,2,1-\mu, \Omega}\right) .
\end{aligned}
$$

In view of the proof of Lemma 5.3.1 from [zaj 5] the first four terms are estimated by

$$
\varepsilon\left|\alpha_{, x x}^{\prime}\right|_{2,1-\mu, \Omega^{t}}^{2}+c(1 / \varepsilon) \sup _{t}\left|\alpha^{\prime}\right|_{2,1-\mu, \Omega}^{2} \varphi\left(\|v\|_{A_{1-\mu}\left(\Omega^{t}\right)}\right)
$$

for any $\varepsilon>0$, where $\varphi$ is an increasing positive function and $\mu \geq 1 / 2$; the fifth term is estimated by

and the sixth by

$$
c\left|\alpha_{2} / r\right|_{10 / 3, \Omega^{t}}\|h\|_{2,-\mu, \Omega^{t}}
$$

$$
c\|h\|_{2,-\mu, \Omega^{t}} .
$$

In view of the assumptions the ninth term is estimated by

$$
c\|w\|_{2,1-\mu, \Omega^{t}}
$$

and the tenth term by

$$
I \equiv c\left(\|h\|_{2,-\mu, \Omega^{t}}+\|w\|_{2,1-\mu, \Omega^{t}}\right) .
$$

To show the above bound we consider

$$
\left\|\beta_{1} h_{r}+\beta_{2} h_{z}\right\|_{1 / 2,2,1-\mu, S^{t}} \leq c\left\|\beta_{1} h_{r}+\beta_{2} h_{z}\right\|_{1,2,1-\mu, \Omega^{t}} \equiv I_{1} .
$$

To examine $I_{1}$ it is enough to consider

$$
\left\|\beta_{1} h_{r}\right\|_{1,2,1-\mu, \Omega^{t}} \leq\left|\nabla \beta_{1} h_{r}\right|_{2,1-\mu, \Omega^{t}}+\left|\beta_{1} \nabla h_{r}\right|_{2,1-\mu, \Omega^{t}}+\left|\beta_{1} h_{r}\right|_{2,1-\mu, \Omega^{t}} \equiv I_{2} .
$$

Since $\left|\nabla \beta_{i}\right| \leq c / r^{3}, i=1,2$, we have $\left|\nabla \beta_{1} h_{r}\right|_{2,1-\mu, \Omega^{t}} \leq c\left|h_{r}\right|_{\left.2,-(2+\mu), \Omega^{t}\right)}$ and for $\left|\beta_{i}\right| \leq$ $c / r^{2}, i=1,2$, we get $\left|\beta_{1} \nabla h_{r}\right|_{2,1-\mu, \Omega^{t}} \leq c\left|\nabla h_{r}\right|_{2,-(1+\mu), \Omega^{t}}$. Hence $I_{1} \leq c\|h\|_{2,-\mu, \Omega^{t}}$. Let us consider

$$
\left\|\beta_{3} w_{, r}+\beta_{4} w_{, z}\right\|_{1 / 2,2,1-\mu, S^{t}} \leq c\left\|\beta_{3} w_{, r}+\beta_{4} w_{, z}\right\|_{1,2,1-\mu, \Omega^{t}} \equiv I_{3} .
$$

Since $\left|\beta_{i}\right| \leq c,\left|\nabla \beta_{i}\right| \leq c / r, i=3,4$, we have

$$
\begin{aligned}
I_{3} & \leq \sum_{i=3}^{4}\left(\left|\beta_{i} w_{, x x}\right|_{2,1-\mu, \Omega^{t}}+\left|\beta_{i} w_{, x}\right|_{2,1-\mu, \Omega^{t}}+\left|\nabla \beta_{i} w\right|_{2,1-\mu, \Omega^{t}}\right) \\
& \leq c\left(\left|w_{, x x}\right|_{2,1-\mu, \Omega^{t}}+\left|w_{, x}\right|_{2,-\mu, \Omega^{t}}\right) \leq c\|w\|_{2,1-\mu, \Omega^{t}} .
\end{aligned}
$$

Finally,

$$
\left\|\beta_{5} w\right\|_{1 / 2,2,1-\mu, S^{t}} \leq c\left\|\beta_{5} w\right\|_{1,2,1-\mu, \Omega^{t}} \equiv I_{4} .
$$

Hence for $\left|\beta_{5}\right| \leq c / r,\left|\nabla \beta_{5}\right| \leq c / r^{2}$ we obtain

$$
\begin{aligned}
I_{4} & \leq c\left(\left|\beta_{5} \nabla w\right|_{2,1-\mu, \Omega^{t}}+\left|\nabla \beta_{5} w\right|_{2,1-\mu, \Omega^{t}}\right) \\
& \leq c|\nabla w|_{2,-\mu, \Omega^{t}}+|w|_{\left.2,-(1+\mu), \Omega^{t}\right)} \leq c\|w\|_{2,1-\mu, \Omega^{t}} .
\end{aligned}
$$

Therefore the bound $I$ follows. In view of the above considerations we obtain (4.12). This concludes the proof. 
From (4.12) we see that $\sup _{t}\left|\alpha^{\prime}\right|_{2,1-\mu, \Omega}$ and $\left|\alpha_{1}\right|_{2,-(1+\mu), \mid \Omega^{t}}$ must be estimated. Hence we need

LEMMA 4.4. Assume that $F^{\prime} \in L_{2}\left(\Omega^{t}\right), v \in L_{2}\left(0, t ; W_{2,1-\mu}^{3}(\Omega)\right), h \in H_{-1}^{2,1}\left(\Omega^{t}\right), w \in$ $H_{1-\mu}^{2,1}\left(\Omega^{t}\right), \mu \in(1 / 2,1), \alpha_{10}, \alpha_{30} \in W_{2,1-\mu}^{1}(\Omega),\left|a_{1} / r\right| \leq c,\left|\nabla\left(a_{1} / r\right)\right| \leq c r^{-1},\left|\nabla^{2}\left(a_{1} / r\right)\right| \leq$ $c r^{-2},\left|\beta_{i}\right| \leq c / r^{3-\mu},\left|\nabla \beta_{i}\right| \leq c r^{-(4-\mu)} i=1,2,\left|\beta_{i}\right| \leq c,\left|\nabla \beta_{i}\right| \leq c r^{-1}, i=3,4,\left|\nabla \beta_{5}\right| \leq$ $c r^{-2},\left|\beta_{5}\right| \leq c / r, t \in(0, T)$. Asume that $S$ contains a part $S_{1}$ where $a_{1} \geq a_{0}>0$ such that any point of $\Omega$ can be reached from points of $S_{1}$ by a curve. Assume that $S_{2}$ is a part of $S$ such that $a_{2} \neq 0$. Assume that $\left|a_{1} / a_{2}\right| \leq c r^{2},\left|\nabla\left(a_{1} / a_{2}\right)\right| \leq c r,\left|\nabla^{2}\left(a_{1} / a_{2}\right)\right| \leq c$ on $S_{2}$. Then solutions of (1.7) satisfy the estimate

$$
\begin{aligned}
\left|\alpha_{1}\right|_{2, \Omega}^{2}+ & \left|\alpha_{3}\right|_{2, \Omega}^{2}+\nu\left(\left|\alpha_{1, x}\right|_{2, \Omega^{t}}^{2}+\left|\alpha_{3, x}\right|_{2, \Omega^{t}}^{2}\right)+\nu\left|\alpha_{1}\right|_{2,-1, \Omega^{t}}^{2} \\
\leq & c \exp \left(\int_{0}^{t}\left\|v\left(t^{\prime}\right)\right\|_{3,2,1-\mu, \Omega}^{2} d t^{\prime}\right)\left[\left|\alpha_{2}\right|_{10 / 3, \Omega^{t}}^{2}\|h\|_{2,-1, \Omega^{t}}^{2}+\|w\|_{2,1-\mu, \Omega^{t}}^{2}\right. \\
& +\|h\|_{2,-1, \Omega^{t}}^{2}+\left|F_{1}\right|_{2, \Omega^{t}}^{2}+\left|F_{3}\right|_{2, \Omega^{t}}^{2}+\left|\alpha_{1}(0)\right|_{2, \Omega}^{2}+\left|\alpha_{3}(0)\right|_{2, \Omega}^{2} \\
& \left.+\left(\|w\|_{2,1-\mu, \Omega^{t}}^{2}+\|h\|_{2,-1, \Omega^{t}}^{2}\right) \int_{0}^{t}\left\|v\left(t^{\prime}\right)\right\|_{3,2,1-\mu, \Omega}^{2} d t^{\prime}\right] .
\end{aligned}
$$

Proof. First we obtain the energy type estimate for solutions of (1.7). For this purpose we introduce functions $\widetilde{\alpha}_{1}, \widetilde{\alpha}_{3}$ as solutions of the problem

$$
\begin{array}{ll}
\widetilde{\alpha}_{1, t}-\nu \Delta \widetilde{\alpha}_{1}=0, & \\
\widetilde{\alpha}_{3, t}-\nu \Delta \widetilde{\alpha}_{3}=0, & \text { on } S, \\
a_{2} \widetilde{\alpha}_{1}-a_{1} \widetilde{\alpha}_{3}=-\frac{2 a_{1}}{r} w+\frac{\gamma}{\nu} w \equiv g_{1} & \text { on } S, \\
\left(a_{1} \widetilde{\alpha}_{1}+a_{2} \widetilde{\alpha}_{3}\right)_{, n}=\beta_{1} h_{r}+\beta_{2} h_{z}+\beta_{3} w_{, r}+\beta_{4} w_{, z}+\beta_{5} w \equiv g_{2} & \\
\left.\widetilde{\alpha}_{1}\right|_{t=0}=0, & \\
\left.\widetilde{\alpha}_{3}\right|_{t=0}=0 . &
\end{array}
$$

Define the functions

$$
\bar{\alpha}_{1}=\alpha_{1}-\widetilde{\alpha}_{1}, \quad \bar{\alpha}_{3}=\alpha_{3}-\widetilde{\alpha}_{3} .
$$

Then problem (1.7) takes the form

$$
\begin{aligned}
& \bar{\alpha}_{1, t}+v \cdot \nabla \bar{\alpha}_{1}-\left(\bar{\alpha}_{1} v_{r, r}+\bar{\alpha}_{3} v_{r, z}+\frac{\alpha_{2}}{r} h_{r}\right)+\frac{2 \nu}{r^{2}} \alpha_{2, \varphi}+\nu \frac{\bar{\alpha}_{1}}{r^{2}}-\nu \Delta \bar{\alpha}_{1} \\
& \quad=F_{1}-v \cdot \nabla \widetilde{\alpha}_{1}+\left(\widetilde{\alpha}_{1} v_{r, r}+\widetilde{\alpha}_{3} v_{r, z}\right)-\nu \frac{\widetilde{\alpha}_{1}}{r^{2}} \\
& \bar{\alpha}_{3, t}+v \cdot \nabla \bar{\alpha}_{3}-\left(\bar{\alpha}_{1} v_{z, r}+\bar{\alpha}_{3} v_{z, z}+\frac{\alpha_{2}}{r} h_{z}\right)-\nu \Delta \bar{\alpha}_{3} \\
& \quad=F_{3}-v \cdot \nabla \widetilde{\alpha}_{3}+\left(\widetilde{\alpha}_{1} v_{z, r}+\widetilde{\alpha}_{3} v_{z, z}\right), \\
& a_{2} \bar{\alpha}_{1}-a_{1} \bar{\alpha}_{3}=0 \quad \text { on } S^{t} \\
& \left(a_{1} \bar{\alpha}_{1}+a_{2} \bar{\alpha}_{3}\right)_{, n}=0 \quad \text { on } S^{t}, \\
& \left.\bar{\alpha}_{1}\right|_{t=0}=\alpha_{1}(0), \\
& \left.\bar{\alpha}_{3}\right|_{t=0}=\alpha_{3}(0) .
\end{aligned}
$$


Solving (4.15) we have

$$
\begin{aligned}
\left\|\widetilde{\alpha}_{1}\right\|_{2,2,1}-\mu, \Omega^{t} & +\left\|\widetilde{\alpha}_{3}\right\|_{2,2,1-\mu, \Omega^{t}} \leq c\left[\left\|\frac{a_{1}}{r} w\right\|_{3 / 2,2,1-\mu, S^{t}}\right. \\
& +\left\|\beta_{1} h_{r}+\beta_{2} h_{z}+\beta_{3} w_{, r}+\beta_{4} w_{, z}+\beta_{5} w\right\|_{1 / 2,2,1-\mu, S^{t}}+\left\|\alpha_{1}(0)\right\|_{1,2,1-\mu, \Omega} \\
& \left.+\left\|\alpha_{3}(0)\right\|_{1,2,1-\mu, \Omega}\right] .
\end{aligned}
$$

Now we examine the norms on the r.h.s. of (4.18). Assuming $\left|a_{1} / r\right| \leq c,\left|\nabla\left(a_{1} / r\right)\right| \leq c$, $\left|\nabla^{2}\left(a_{1} / r\right)\right| \leq c / r^{2}$ we find that the first term on the r.h.s. is bounded by $c\|w\|_{2,1-\mu, \Omega^{t}}$. This behaviour is imposed in a neighbourhood of the point where the $z$ axis crosses $S$. Assuming $\left|\beta_{i}\right| \leq c / r^{3-\mu},\left|\nabla \beta_{i}\right| \leq c / r^{4-\mu}, i=1,2$, we have

$$
\left\|\beta_{1} h_{r}+\beta_{2} h_{z}\right\|_{1 / 2,2,1-\mu, S^{t}} \leq c\|h\|_{2,-1, \Omega^{t}} .
$$

Finally, assuming $\left|\beta_{i}\right| \leq c,\left|\nabla \beta_{i}\right| \leq c / r, i=3,4,\left|\beta_{5}\right| \leq c / r,\left|\nabla \beta_{5}\right| \leq c / r^{2}$ we get

$$
\left\|\beta_{3} w_{, r}+\beta_{4} w_{, z}+\beta_{5} w\right\|_{1 / 2,2,1-\mu, S^{t}} \leq c\|w\|_{2,1-\mu, \Omega^{t}} .
$$

Hence, (4.18) takes the form

$$
\begin{aligned}
\left\|\widetilde{\alpha}_{1}\right\|_{2,2,1-\mu, \Omega^{t}}+\left\|\widetilde{\alpha}_{3}\right\|_{2,2,1-\mu, \Omega^{t}} \leq c( & \|h\|_{2,-1, \Omega^{t}}+\|w\|_{2,1-\mu, \Omega^{t}} \\
& \left.+\left\|\alpha_{1}(0)\right\|_{1,2,1-\mu, \Omega}+\left\|\alpha_{3}(0)\right\|_{1,2,1-\mu, \Omega}\right) .
\end{aligned}
$$

Now we obtain an energy estimate for solutions of (4.17). Multiplying $(4.17)_{1}$ by $\bar{\alpha}_{1}$, $(4.17)_{2}$ by $\bar{\alpha}_{3}$, integrating the results over $\Omega$ and adding yields

$$
\begin{aligned}
\frac{1}{2} \frac{d}{d t}\left(\left|\bar{\alpha}_{1}\right|_{2, \Omega}^{2}+\left|\bar{\alpha}_{3}\right|_{2, \Omega}^{2}\right) & -\int_{\Omega}\left[\bar{\alpha}_{1}^{2} v_{r, r}+\bar{\alpha}_{1} \bar{\alpha}_{3}\left(v_{r, z}+v_{z, r}\right)+\bar{\alpha}_{3}^{2} v_{z, z}\right] d x \\
& -\int_{\Omega} \frac{\alpha_{2}}{r}\left(h_{r} \bar{\alpha}_{1}+h_{z} \bar{\alpha}_{3}\right) d x+2 \nu \int_{\Omega} \frac{1}{r^{2}} \alpha_{2, \varphi} \bar{\alpha}_{1} d x+\nu\left|\bar{\alpha}_{1}\right|_{2,-1, \Omega}^{2} \\
& -\nu \int_{\Omega}\left(\Delta \bar{\alpha}_{1} \bar{\alpha}_{1}+\Delta \bar{\alpha}_{3} \bar{\alpha}_{3}\right) d x=\int_{\Omega}\left(F_{1} \bar{\alpha}_{1}+F_{3} \bar{\alpha}_{3}\right) d x \\
& -\int_{\Omega}\left(v \cdot \nabla \widetilde{\alpha}_{1} \bar{\alpha}_{1}+v \cdot \nabla \widetilde{\alpha}_{3} \bar{\alpha}_{3}\right) d x-\nu \int_{\Omega} \frac{\widetilde{\alpha}_{1}}{r^{2}} \bar{\alpha}_{1} d x \\
& +\int_{\Omega}\left[\left(\widetilde{\alpha}_{1} v_{r, r}+\widetilde{\alpha}_{3} v_{r, z}\right) \bar{\alpha}_{1}+\left(\widetilde{\alpha}_{1} v_{z, r}+\widetilde{\alpha}_{3} v_{z, z}\right) \bar{\alpha}_{3}\right] d x .
\end{aligned}
$$

The term involving laplacians takes the form

$$
-\int_{S}\left[\bar{\alpha}_{1, n} \bar{\alpha}_{1}+\bar{\alpha}_{3, n} \bar{\alpha}_{3}\right] d S+\left|\bar{\alpha}_{1, x}\right|_{2, \Omega}^{2}+\left|\bar{\alpha}_{3, x}\right|_{2, \Omega}^{2},
$$

where in view of the boundary conditions $(4.17)_{3,4}$ the boundary term vanishes.

For completeness we show how to prove this. Let $S_{2}=\left\{x \in S: a_{2} \neq 0\right\}$. From $(4.17)_{4}$ we have

$$
I=\bar{\alpha}_{1, n} \bar{\alpha}_{1}+\bar{\alpha}_{3, n} \bar{\alpha}_{3}=\bar{\alpha}_{1, n} \bar{\alpha}_{1}-\frac{a_{1}}{a_{2}} \bar{\alpha}_{1, n} \bar{\alpha}_{3}-\frac{1}{a_{2}}\left(a_{1, n} \bar{\alpha}_{1}+a_{2, n} \bar{\alpha}_{3}\right) \bar{\alpha}_{3} .
$$


Employing $(4.17)_{3}$ yields

$$
I=-\frac{1}{a_{2}}\left(a_{1, n} \bar{\alpha}_{1}+a_{2, n} \bar{\alpha}_{3}\right) \bar{\alpha}_{3}
$$

Using $(4.17)_{3}$ again gives

$$
I=-\frac{1}{a_{2}^{2}}\left(a_{1, n} a_{1}+a_{2, n} a_{2}\right) \bar{\alpha}_{3}^{2}=0
$$

because $a_{1}^{2}+a_{2}^{2}=1$.

Similarly, we show that $I(x)=0$ for $x \in S_{1}=\left\{x \in S: a_{1} \neq 0\right\}$.

The second term on the l.h.s. of (4.20) is bounded by

$$
\varepsilon\left(\left|\bar{\alpha}_{1}\right|_{2, \Omega}^{2}+\left|\bar{\alpha}_{3}\right|_{2, \Omega}^{2}\right)+c(1 / \varepsilon)\|v\|_{3,2,1-\mu, \Omega}^{2}\left(\left|\bar{\alpha}_{1}\right|_{2, \Omega}^{2}+\left|\bar{\alpha}_{3}\right|_{2, \Omega}^{2}\right)
$$

for any $\varepsilon>0$. The third term is estimated by

$$
\varepsilon\left|\bar{\alpha}_{1}\right|_{2,-1, \Omega}^{2}+c(1 / \varepsilon) \int_{\Omega} \alpha_{2}^{2} h^{2} d x
$$

and the fourth by

$$
\varepsilon\left|\bar{\alpha}_{1}\right|_{2,-1, \Omega}^{2}+c(1 / \varepsilon)\|h\|_{2,-1, \Omega}^{2} .
$$

We bound the first term on the r.h.s. of (4.20) by

$$
\varepsilon\left(\left|\bar{\alpha}_{1}\right|_{2, \Omega}^{2}+\left|\bar{\alpha}_{3}\right|_{2, \Omega}^{2}\right)+c(1 / \varepsilon)\left(\left|F_{1}\right|_{2, \Omega}^{2}+\left|F_{3}\right|_{2, \Omega}^{2}\right)
$$

the second by

$$
\varepsilon\left(\left|\nabla \bar{\alpha}_{1}\right|_{2, \Omega}^{2}+\left|\nabla \bar{\alpha}_{3}\right|_{2, \Omega}^{2}\right)+c(1 / \varepsilon)|v|_{\infty, \Omega}^{2}\left(\left|\widetilde{\alpha}_{1}\right|_{2, \Omega}^{2}+\left|\widetilde{\alpha}_{3}\right|_{2, \Omega}^{2}\right),
$$

the third by

and finally the last by

$$
\varepsilon\left|\bar{\alpha}_{1}\right|_{2,-1, \Omega}^{2}+c(1 / \varepsilon)\left|\widetilde{\alpha}_{1}\right|_{2,-1, \Omega}^{2}
$$

$$
\varepsilon\left(\left|\bar{\alpha}_{1}\right|_{2, \Omega}^{2}+\left|\bar{\alpha}_{3}\right|_{2, \Omega}^{2}\right)+c(1 / \varepsilon)\left|v_{, x}\right|_{\infty, \Omega}^{2}\left(\left|\widetilde{\alpha}_{1}\right|_{2, \Omega}^{2}+\left|\widetilde{\alpha}_{3}\right|_{2, \Omega}^{2}\right) .
$$

Summarizing the above results we obtain

$$
\begin{aligned}
\frac{1}{2} \frac{d}{d t}\left(\left|\bar{\alpha}_{1}\right|_{2, \Omega}^{2}+\right. & \left.\left|\bar{\alpha}_{3}\right|_{2, \Omega}^{2}\right)+\frac{\nu}{2}\left(\left|\bar{\alpha}_{1, x}\right|_{2, \Omega}^{2}+\left|\bar{\alpha}_{3, x}\right|_{2, \Omega}^{2}\right)+\frac{\nu}{2}\left|\bar{\alpha}_{1}\right|_{2,-1, \Omega}^{2} \\
\leq & c(1 / \varepsilon)\|v\|_{3,2,1-\mu, \Omega}^{2}\left(\left|\bar{\alpha}_{1}\right|_{2, \Omega}^{2}+\left|\bar{\alpha}_{3}\right|_{2, \Omega}^{2}\right) \\
& +c(1 / \varepsilon)\left(\int_{\Omega} \alpha_{2}^{2} h^{2} d x+\|h\|_{2,-1, \Omega}^{2}+\left|F_{1}\right|_{2, \Omega}^{2}+\left|F_{3}\right|_{2, \Omega}^{2}\right) \\
& +c(1 / \varepsilon)\|v\|_{3,2,1-\mu, \Omega}^{2}\left(\left|\widetilde{\alpha}_{1}\right|_{2, \Omega}^{2}+\left|\widetilde{\alpha}_{3}\right|_{2, \Omega}^{2}\right)+c\left|\widetilde{\alpha}_{1}\right|_{2,-1, \Omega}^{2}+\varepsilon\left|\bar{\alpha}_{3}\right|_{2, \Omega}^{2} .
\end{aligned}
$$

To estimate the last term on the r.h.s. of (4.21) we use the Poincaré inequality. For this purpose we assume that there exists a part $S_{1}$ of the boundary $S$, where $a_{1}$ is separated from zero, that is, there exists a positive number $a_{0}$ such that $\left|a_{1}\right| \geq a_{0}$ on $S_{1}$. Moreover we assume that $S_{1}$ is so large that any point of $\Omega$ can be reached by a straight line from a point of $S_{1}$. An example is a domain close to a cylinder but with a smooth boundary.

We write the boundary condition $(4.17)_{3}$ in the form

$$
\bar{\alpha}_{3}=\frac{a_{2}}{a_{1}} \bar{\alpha}_{1},
$$


and we consider (4.22) on $S_{1}$ only. Then we introduce a function $\widetilde{\alpha}_{3}^{\prime}$ by

$$
\left.\widetilde{\alpha}_{3}^{\prime}\right|_{S_{1}}=\left.\frac{a_{2}}{a_{1}} \bar{\alpha}_{1}\right|_{S_{1}}
$$

Then $\alpha_{3}^{\prime \prime}=\bar{\alpha}_{3}-\widetilde{\alpha}_{3}^{\prime}$ is such that

$$
\left.\alpha_{3}^{\prime \prime}\right|_{S_{1}}=0
$$

By the Poincaré inequality we have

$$
\begin{aligned}
\left|\bar{\alpha}_{3}\right|_{2, \Omega} & \leq\left|\alpha_{3}^{\prime \prime}\right|_{2, \Omega}+\left|\widetilde{\alpha}_{3}^{\prime}\right|_{2, \Omega}^{2} \leq c\left|\nabla \alpha_{3}^{\prime \prime}\right|_{2, \Omega}+\left|\widetilde{\alpha}_{3}^{\prime}\right|_{2, \Omega} \\
& \leq c\left|\nabla \bar{\alpha}_{3}\right|_{2, \Omega}+c\left\|\widetilde{\alpha}_{3}^{\prime}\right\|_{1, \Omega} \leq c\left|\nabla \bar{\alpha}_{3}\right|_{2, \Omega}+c\left\|\bar{\alpha}_{1}\right\|_{1, \Omega} .
\end{aligned}
$$

Using (4.25) in (4.21) yields

$$
\begin{aligned}
\frac{d}{d t}\left(\left|\bar{\alpha}_{1}\right|_{2, \Omega}^{2}+\left|\bar{\alpha}_{3}\right|_{2, \Omega}^{2}\right)+\nu(\mid & \left.\left.\bar{\alpha}_{1, x}\right|_{2, \Omega} ^{2}+\left|\bar{\alpha}_{3, x}\right|_{2, \Omega}^{2}\right)+\nu\left|\bar{\alpha}_{1}\right|_{2,-1, \Omega}^{2} \\
\leq & c\|v\|_{3,2,1-\mu, \Omega}^{2}\left(\left|\bar{\alpha}_{1}\right|_{2, \Omega}^{2}+\left|\bar{\alpha}_{3}\right|_{2, \Omega}^{2}\right) \\
& +c\left(\int_{\Omega} \alpha_{2}^{2} h^{2} d x+\|h\|_{2,-1, \Omega}^{2}+\left|F_{1}\right|_{2, \Omega}^{2}+\left|F_{3}\right|_{2, \Omega}^{2}\right) \\
& +c\|v\|_{3,2,1-\mu, \Omega}^{2}\left(\left|\widetilde{\alpha}_{1}\right|_{2, \Omega}^{2}+\left|\widetilde{\alpha}_{3}\right|_{2, \Omega}^{2}\right)+c\left|\widetilde{\alpha}_{1}\right|_{2,-1, \Omega}^{2} .
\end{aligned}
$$

Integrating (4.26) with respect to $t$ yields

$$
\begin{aligned}
\left|\bar{\alpha}_{1}\right|_{2, \Omega}^{2}+ & \left|\bar{\alpha}_{3}\right|_{2, \Omega}^{2}+\nu\left(\left|\bar{\alpha}_{1, x}\right|_{2, \Omega^{t}}^{2}+\left|\bar{\alpha}_{3, x}\right|_{2, \Omega^{t}}^{2}\right)+\nu\left|\bar{\alpha}_{1}\right|_{2,-1, \Omega^{t}}^{2} \\
\leq & c \exp \left(\int_{0}^{t}\left\|v\left(t^{\prime}\right)\right\|_{3,2,1-\mu, \Omega}^{2} d t^{\prime}\right)\left[\left|\alpha_{2}\right|_{10 / 3, \Omega^{t}}^{2}\|h\|_{2,-1, \Omega^{t}}^{2}+\|h\|_{2,-1, \Omega^{t}}^{2}\right. \\
& +\left|F_{1}\right|_{2, \Omega^{t}}^{2}+\left|F_{3}\right|_{2, \Omega^{t}}^{2}+\sup _{t}\left(\left|\widetilde{\alpha}_{1}\right|_{2, \Omega}^{2}+\left|\widetilde{\alpha}_{3}\right|_{2, \Omega}^{2}\right) \int_{0}^{t}\left\|v\left(t^{\prime}\right)\right\|_{3,2,1-\mu, \Omega}^{2} d t^{\prime} \\
& \left.+\left|\widetilde{\alpha}_{1}\right|_{2,-1, \Omega^{t}}^{2}+\left|\bar{\alpha}_{1}(0)\right|_{2, \Omega}^{2}+\left|\bar{\alpha}_{3}(0)\right|_{2, \Omega}^{2}\right] .
\end{aligned}
$$

To estimate the norms of $\widetilde{\alpha}_{1}$ and $\widetilde{\alpha}_{3}$ on the r.h.s. of (4.27) we examine problem (4.15). Multiplying $(4.15)_{1}$ by $\widetilde{\alpha}_{1},(4.15)_{2}$ by $\widetilde{\alpha}_{3}$, integrating the results over $\Omega$ and adding we have

$$
\begin{aligned}
\frac{1}{2} \frac{d}{d t}\left(\left|\widetilde{\alpha}_{1}\right|_{2, \Omega}^{2}+\left|\widetilde{\alpha}_{3}\right|_{2, \Omega}^{2}\right)+\nu\left(\left|\nabla \widetilde{\alpha}_{1}\right|_{2, \Omega}^{2}+\left|\nabla \widetilde{\alpha}_{3}\right|_{2, \Omega}^{2}\right) & \\
& =\nu \int_{S}\left(\widetilde{\alpha}_{1, n} \widetilde{\alpha}_{1}+\widetilde{\alpha}_{3, n} \widetilde{\alpha}_{3}\right) d S \equiv I .
\end{aligned}
$$

To estimate $I$ we divide $S$ into two parts $S_{1}, S_{2}$ such that $S=S_{1} \cup S_{2}$, where $a_{1} \neq 0$ on $S_{1}$ and $a_{2} \neq 0$ on $S_{2}$. Then we have

$$
I=\nu \int_{S_{1}}\left(\widetilde{\alpha}_{1, n} \widetilde{\alpha}_{1}+\widetilde{\alpha}_{3, n} \widetilde{\alpha}_{3}\right) d S_{1}+\nu \int_{S_{2}}\left(\widetilde{\alpha}_{1, n} \widetilde{\alpha}_{1}+\widetilde{\alpha}_{3, n} \widetilde{\alpha}_{3}\right) d S_{2} \equiv I_{1}+I_{2} .
$$

Let us consider $I_{1}$. In view of the boundary conditions $(4.15)_{3,4}$ we have

$$
\widetilde{\alpha}_{3}=\frac{a_{2}}{a_{1}} \widetilde{\alpha}_{1}-\frac{1}{a_{1}} g_{1}, \quad \widetilde{\alpha}_{1, n}=\frac{1}{a_{1}}\left[g_{2}-a_{2} \widetilde{\alpha}_{3, n}+\frac{a_{2, n}}{a_{1}} g_{1}\right],
$$


SO

and

$$
I_{1}=\nu \int_{S_{1}} \frac{1}{a_{1}}\left[\left(g_{2}+\frac{a_{2, n}}{a_{1}} g_{1}\right) \widetilde{\alpha}_{1}-\widetilde{\alpha}_{3, n} g_{1}\right] d S_{1}
$$

$$
\begin{aligned}
\left|I_{1}\right| \leq & \varepsilon\left(\left|\widetilde{\alpha}_{3, n}\right|_{2, S_{1}}^{2}+\left|\widetilde{\alpha}_{1}\right|_{2, S_{1}}^{2}\right)+c(1 / \varepsilon)\left(\left|g_{1}\right|_{2, S_{1}}^{2}+\left|g_{2}\right|_{2, S_{1}}^{2}\right) \\
\leq & \varepsilon\left(\left|\widetilde{\alpha}_{3, x x}\right|_{2,1-\mu, \Omega}^{2}+\left|\widetilde{\alpha}_{3}\right|_{2, \Omega}^{2}+\left|\widetilde{\alpha}_{1, x}\right|_{2, \Omega}^{2}+\left|\widetilde{\alpha}_{1}\right|_{2, \Omega}^{2}\right) \\
& +c(1 / \varepsilon)\left(\left|g_{1}\right|_{2, S_{1}}^{2}+\left|g_{2}\right|_{2, S_{1}}^{2}\right) .
\end{aligned}
$$

Considering $I_{2}$ we calculate

$$
\widetilde{\alpha}_{1}=\frac{a_{1}}{a_{2}} \widetilde{\alpha}_{3}+\frac{1}{a_{2}} g_{1}, \quad \widetilde{\alpha}_{3, n}=\frac{1}{a_{2}}\left[g_{2}-a_{1} \widetilde{\alpha}_{1, n}-\frac{a_{1, n}}{a_{2}} g_{1}\right],
$$

and

$$
I_{2}=\nu \int_{S_{2}} \frac{1}{a_{2}}\left[\widetilde{\alpha}_{1, n} g_{1}+\left(g_{2}-\frac{a_{1, n}}{a_{2}} g_{1}\right) \widetilde{\alpha}_{3}\right] d S_{2}
$$

so

$$
\begin{aligned}
\left|I_{2}\right| \leq & \varepsilon\left(\left|\widetilde{\alpha}_{1, x x}\right|_{2,1-\mu, \Omega}^{2}+\left|\widetilde{\alpha}_{1}\right|_{2, \Omega}^{2}+\left|\widetilde{\alpha}_{3, x}\right|_{2, \Omega}^{2}+\left|\widetilde{\alpha}_{3}\right|_{2, \Omega}^{2}\right) . \\
& +c(1 / \varepsilon)\left(\left|g_{1}\right|_{2, S_{1}}^{2}+\left|g_{2}\right|_{2, S_{2}}^{2}\right) .
\end{aligned}
$$

Applying potential theory to (4.15) yields

$$
\left\|\widetilde{\alpha}_{1}\right\|_{2,2,1-\mu, \Omega^{t}}+\left\|\widetilde{\alpha}_{3}\right\|_{2,2,1-\mu, \Omega^{t}} \leq c\left(\left\|g_{1}\right\|_{3 / 2,2,1-\mu, S^{t}}+\left\|g_{2}\right\|_{1 / 2,2,1-\mu, S^{t}}\right) .
$$

From (4.28)-(4.31) we have

$$
\begin{aligned}
\left|\widetilde{\alpha}_{1}\right|_{2, \Omega}^{2}+ & \left|\widetilde{\alpha}_{3}\right|_{2, \Omega}^{2}+\left\|\widetilde{\alpha}_{1}\right\|_{2,2,1-\mu, \Omega^{t}}^{2}+\left\|\widetilde{\alpha}_{3}\right\|_{2,2,1-\mu, \Omega^{t}}^{2} \\
& \leq c\left(\left\|g_{1}\right\|_{3 / 2,1-\mu, S^{t}}^{2}+\left\|g_{2}\right\|_{1 / 2,1-\mu, S^{t}}^{2}\right) \leq c\left(\|w\|_{2,1-\mu, \Omega^{t}}^{2}+\|h\|_{2,-1, \Omega^{t}}^{2}\right) .
\end{aligned}
$$

To estimate the norm $\left|\widetilde{\alpha}_{1}\right|_{2,-1, \Omega^{t}}$ from the r.h.s. of (4.27) we have to examine the behaviour of $\widetilde{\alpha}_{1}$ in neighbourhoods of points $r=0, z=-a$ and $r=0, z=a$. Assume that $\psi_{, r}$ vanishes only at these points and $\psi_{, z}$ is different from zero in some neighbourhoods. We denote these neighbourhoods of points of $S$ by $S_{2}, S_{2}=\left\{x \in S: a_{2} \neq 0\right\}$. Let us restrict our considerations to the point $r=0, z=a$, because near $r=0, z=-a$ we argue similarly. Let us denote the point $r=0, z=a$ by $z_{0}$. Let $B\left(z_{0}, \lambda\right)$ be the ball of radius $\lambda$ and with centre at $z_{0}$. Let $\zeta=\zeta(r, z)$ be a smooth function such that $\zeta(r, z)=1$ for $(r, z) \in B\left(z_{0}, \lambda\right) \cap \bar{\Omega}$ and $\zeta(r, z)=0$ for $(r, z) \notin B\left(z_{0}, 2 \lambda\right) \cap \bar{\Omega}$. Defining $\widetilde{\alpha}_{1}^{\prime}=\widetilde{\alpha}_{1} \zeta$, $g_{1}^{\prime}=g_{1} \zeta$ we obtain from $(4.15)_{1,3}$ the following problem:

$$
\begin{aligned}
& \widetilde{\alpha}_{1, t}^{\prime}-\nu \Delta \widetilde{\alpha}_{1}^{\prime}=-2 \nu \nabla \widetilde{\alpha}_{1} \nabla \zeta-\nu \Delta \zeta \widetilde{\alpha}_{1}, \\
& \left.\widetilde{\alpha}_{1}^{\prime}\right|_{S_{2} \cap \operatorname{supp} \zeta}=\frac{a_{1}}{a_{2}} \widetilde{\alpha}_{3}^{\prime}+\frac{1}{a_{2}} g_{1}^{\prime} \text {, } \\
& \left.\widetilde{\alpha}_{1}^{\prime}\right|_{t=0}=0 \text {, }
\end{aligned}
$$

where $\widetilde{\alpha}_{3}^{\prime}=\widetilde{\alpha}_{3} \zeta$.

Assuming $\left|a_{1} / a_{2}\right| \leq c r^{2},\left|\nabla\left(a_{1} / a_{2}\right)\right| \leq c r,\left|\nabla^{2}\left(a_{1} / a_{2}\right)\right| \leq c$ in a neighbourhood of $r=0, z=a$ we get for solutions of (4.33) the estimate

$$
\begin{aligned}
\left\|\widetilde{\alpha}_{1}^{\prime}\right\|_{2,1-\mu, \Omega^{t}} & \leq c\left(\left|\nabla \widetilde{\alpha}_{1} \nabla \zeta\right|_{2,1-\mu, \Omega^{t}}+\left|\Delta \zeta \widetilde{\alpha}_{1}\right|_{2,1-\mu, \Omega^{t}}+\left\|\widetilde{\alpha}_{3}^{\prime}\right\|_{2,2,1-\mu, \Omega^{t}}+\left\|g_{1}^{\prime}\right\|_{3 / 2,1-\mu, S_{2}^{t}}\right) \\
& \leq c\left(\left\|\widetilde{\alpha}_{1}\right\|_{2,2,1-\mu, \Omega^{t}}+\left\|\widetilde{\alpha}_{3}\right\|_{2,2,1-\mu, \Omega^{t}}+\|w\|_{2,1-\mu, \Omega^{t}}\right) .
\end{aligned}
$$


In view of (4.32) and properties of $\zeta$ we have

$$
\left\|\widetilde{\alpha}_{1}\right\|_{2,1-\mu, \Omega^{t}} \leq c\left(\left\|\widetilde{\alpha}_{3}\right\|_{2,2,1-\mu, \Omega^{t}}+\|w\|_{2,1-\mu, \Omega^{t}}+\|h\|_{2,-1, \Omega^{t}}\right) .
$$

In virtue of (4.32) and (4.34) we obtain

$$
\left|\widetilde{\alpha}_{1}\right|_{2,-(1+\mu), \Omega^{t}} \leq c\left(\|w\|_{2,1-\mu, \Omega^{t}}+\|h\|_{2,-1, \Omega^{t}}\right) .
$$

Using (4.32) and (4.35) in (4.27) yields

$$
\begin{aligned}
& \left|\bar{\alpha}_{1}\right|_{2, \Omega}^{2}+\left|\bar{\alpha}_{3}\right|_{2, \Omega}^{2}+\nu\left(\left|\bar{\alpha}_{1, x}\right|_{2, \Omega^{t}}^{2}+\left|\bar{\alpha}_{3, x}\right|_{2, \Omega^{t}}^{2}\right)+\nu\left|\bar{\alpha}_{1}\right|_{2,-1, \Omega^{t}}^{2} \\
& \leq c \exp \left(\int_{0}^{t}\left\|v\left(t^{\prime}\right)\right\| \|_{3,2,1-\mu, \Omega}^{2} d t^{\prime}\right)\left[\left|\alpha_{2}\right|_{10 / 3, \Omega^{t}}^{2}\|h\|_{2,-1, \Omega^{t}}^{2}+\|h\|_{2,-1, \Omega^{t}}^{2}\right. \\
& \quad+\left|F_{1}\right|_{2, \Omega^{t}}^{2}+\left|F_{3}\right|_{2, \Omega^{t}}^{2}+\left(\|w\|_{2,1-\mu, \Omega^{t}}^{2}+\|h\|_{2,-1, \Omega^{t}}^{2}\right) \int_{0}^{t}\left\|v\left(t^{\prime}\right)\right\|_{3,2,1-\mu, \Omega}^{2} d t^{\prime} \\
& \left.\quad+\|w\|_{2,1-\mu, \Omega^{t}}^{2}+\left|\bar{\alpha}_{1}(0)\right|_{2, \Omega}^{2}+\left|\bar{\alpha}_{3}(0)\right|_{2, \Omega}^{2}\right] .
\end{aligned}
$$

Using (4.32) and (4.35) in (4.36) implies (4.14). This concludes the proof.

Finally, we obtain an estimate for $\left|\alpha_{1}\right|_{2,-(1+\mu), \Omega^{t}}$.

LEMmA 4.5. Assume that $h \in H_{-1}^{2,1}\left(\Omega^{t}\right), w \in H_{1-\mu}^{2,1}\left(\Omega^{t}\right), \alpha_{2} / r \in L_{10 / 3}\left(\Omega^{t}\right), F^{\prime} \in L_{2}\left(\Omega^{t}\right)$, $F^{\prime}=\left(F_{1}, F_{3}\right), F_{1} \in L_{2,-\mu}\left(\Omega^{t}\right), \alpha^{\prime}(0) \in W_{2,1-\mu}^{2}(\Omega), \alpha_{1}(0) \in L_{2,-\mu}(\Omega), v \in A_{1-\mu}\left(\Omega^{t}\right)$, $\mu \in(1 / 2,1),\left|a_{1} / r\right| \leq c, t \leq T$. Assume that $\left|a_{1}\right| \leq c r^{2}$ on $S_{2}=\left\{x \in S: a_{2} \neq 0\right\}$. Then

$$
\begin{aligned}
\left|\alpha_{1, x}\right|_{2,-\mu, \Omega^{t}}+\left|\alpha_{1}\right|_{2,-(1+\mu), \Omega^{t}} \leq & \varphi\left(\|v\|_{A_{1-\mu}\left(\Omega^{t}\right)}\right)\left[\left|\alpha_{2} / r\right|_{10 / 3, \Omega^{t}}\|h\|_{2,-1, \Omega^{t}}\right. \\
& +\|h\|_{2,-1, \Omega^{t}}+\|w\|_{2,1-\mu, \Omega^{t}}+\left|F^{\prime}\right|_{2, \Omega^{t}}+\left|F_{1}\right|_{2,-\mu, \Omega^{t}} \\
& \left.+\left\|\alpha^{\prime}(0)\right\|_{1,2,1-\mu, \Omega}+\left|\alpha_{1}(0)\right|_{2,-\mu, \Omega}\right] .
\end{aligned}
$$

Proof. Since we have already proved estimate (4.35) for $\left|\widetilde{\alpha}_{1}\right|_{2,-(1+\mu), \Omega^{t}}$ and (4.32) for $\left|\widetilde{\alpha}_{1, x}\right|_{2,-\mu, \Omega^{t}}$ we shall restrict ourselves to obtaining an estimate for $\left|\bar{\alpha}_{1}\right|_{2,-(1+\mu), \Omega^{t}}$. Multiplying $(4.17)_{1}$ by $\bar{\alpha}_{1} r^{-2 \mu}$ and integrating over $\Omega$ implies

$$
\begin{aligned}
& \frac{1}{2} \frac{d}{d t}\left|\bar{\alpha}_{1}\right|_{2,-\mu, \Omega}^{2}+\int_{\Omega} v \cdot \nabla \bar{\alpha}_{1} \bar{\alpha}_{1} r^{-2 \mu} d x-\int_{\Omega}\left(\bar{\alpha}_{1} v_{r, r}+\bar{\alpha}_{3} v_{r, z}+\frac{\alpha_{2}}{r} h_{r}\right) \bar{\alpha}_{1} r^{-2 \mu} d x \\
& \quad+2 \nu \int_{\Omega} \frac{1}{r^{2}}\left(h_{r, z}-h_{z, r}\right) \bar{\alpha}_{1} r^{-2 \mu} d x+\nu\left|\bar{\alpha}_{1}\right|_{2,-(1+\mu), \Omega}^{2}-\nu \int_{\Omega} \Delta \bar{\alpha}_{1} \bar{\alpha}_{1} r^{-2 \mu} d x \\
& =\int_{\Omega} F_{1} \bar{\alpha}_{1} r^{-2 \mu} d x-\int_{\Omega} v \cdot \nabla \widetilde{\alpha}_{1} \bar{\alpha}_{1} r^{-2 \mu} d x+\int_{\Omega}\left(\widetilde{\alpha}_{1} v_{r, r}+\widetilde{\alpha}_{3} v_{r, z}\right) \bar{\alpha}_{1} r^{-2 \mu} d x \\
& -\nu \int_{\Omega} \frac{\widetilde{\alpha}_{1}}{r^{2}} \bar{\alpha}_{1} r^{-2 \mu} d x .
\end{aligned}
$$

Now we examine the particular terms in (4.38). The second term on the l.h.s. equals

$$
\frac{1}{2} \int_{\Omega} v \cdot \nabla \bar{\alpha}_{1}^{2} r^{-2 \mu} d x=\frac{1}{2} \int_{\Omega} v \cdot \nabla\left(\bar{\alpha}_{1}^{2} r^{-2 \mu}\right) d x+\mu \int_{\Omega} \bar{\alpha}_{1}^{2} r^{-2 \mu-1} v \cdot \nabla r d x,
$$


where the first term vanishes and the second is estimated by

$$
\varepsilon\left|\bar{\alpha}_{1}\right|_{2,-(1+\mu), \Omega}^{2}+c(1 / \varepsilon)|v|_{\infty, \Omega}^{2}\left|\bar{\alpha}_{1}\right|_{2,-\mu, \Omega}^{2}
$$

for any $\varepsilon>0$. The third term in (4.38) is estimated by

$$
\varepsilon\left(\left|\bar{\alpha}_{1}\right|_{2,-\mu, \Omega}^{2}+\left|\bar{\alpha}_{3}\right|_{2,-\mu, \Omega}^{2}\right)+c(1 / \varepsilon)\left|v_{, x}\right|_{\infty, \Omega}^{2}\left|\bar{\alpha}_{1}\right|_{2,-\mu, \Omega}^{2}+c(1 / \varepsilon) \int_{\Omega}\left|\frac{\alpha_{2}}{r}\right|^{2}\left|h_{r}\right|^{2} r^{-2 \mu} d x .
$$

The fourth term on the l.h.s. of (4.38) is bounded by

$$
\varepsilon\left|\bar{\alpha}_{1}\right|_{2,-(1+\mu), \Omega}^{2}+c(1 / \varepsilon)|h|_{2,-1, \Omega}^{2} .
$$

To estimate the last term on the l.h.s. of (4.38) we express boundary conditions $(4.17)_{3,4}$ in the form

$$
\begin{array}{ll}
\bar{\alpha}_{1}=\frac{a_{1}}{a_{2}} \bar{\alpha}_{3} & \text { for } a_{2} \neq 0, \\
\bar{\alpha}_{1, n}=\frac{a_{2}}{a_{1}} \bar{\alpha}_{3, n} & \text { for } a_{1} \neq 0 .
\end{array}
$$

Integrating by parts the term including the laplacian yields

$$
-\nu \int_{\Omega} \operatorname{div}\left(\nabla \bar{\alpha}_{1} \bar{\alpha}_{1} r^{-2 \mu}\right) d x+\nu \int_{\Omega} \bar{\alpha}_{1, x}^{2} r^{-2 \mu} d x-2 \mu \nu \int_{\Omega} \nabla \bar{\alpha}_{1} \bar{\alpha}_{1} r^{-2 \mu-1} \nabla r d x,
$$

where by the Young inequality the last term is bounded by

$$
\varepsilon \frac{\nu}{2}\left|\bar{\alpha}_{1, x}\right|_{2,-\mu, \Omega}^{2}+\frac{2 \mu^{2} \nu}{\varepsilon}\left|\bar{\alpha}_{1}\right|_{2,-(1+\mu), \Omega}^{2}
$$

for any $\varepsilon>0$, and in virtue of (4.39) the first term in (4.40) equals

$$
\begin{aligned}
-\nu \int_{S} \bar{n} \cdot \nabla \bar{\alpha}_{1} \bar{\alpha}_{1} r^{-2 \mu} d S & \\
& =-\nu \int_{S_{1}} \frac{a_{2}}{a_{1}} \bar{\alpha}_{3, n} \bar{\alpha}_{1} r^{-2 \mu} d S_{1}-\nu \int_{S_{2}} \bar{n} \cdot \nabla \bar{\alpha}_{1} \frac{a_{1}}{a_{2}} \bar{\alpha}_{3} r^{-2 \mu} d S_{2} \equiv I_{1}+I_{2},
\end{aligned}
$$

where

$$
\begin{aligned}
& \left|I_{1}\right| \leq \varepsilon\left\|\bar{\alpha}_{3}\right\|_{2,2,1-\mu, \Omega}^{2}+c(1 / \varepsilon)\left\|\bar{\alpha}_{1}\right\|_{1, \Omega}^{2}, \\
& \left|I_{2}\right| \leq \varepsilon\left\|\bar{\alpha}_{1}\right\|_{2,2,1-\mu, \Omega}^{2}+c(1 / \varepsilon)\left\|\bar{\alpha}_{3}\right\|_{1, \Omega}^{2},
\end{aligned}
$$

where we used the fact that $r>0$ on $S_{1}$ and $\left|a_{1} r^{-2}\right| \leq c$ on $S_{2}$.

We estimate the first term on the r.h.s. of (4.38) by

$$
\varepsilon\left|\bar{\alpha}_{1}\right|_{2,-\mu, \Omega}+c(1 / \varepsilon)\left|F_{1}\right|_{2,-\mu, \Omega}^{2},
$$

the second by

and the third by

$$
\varepsilon\left|\nabla \widetilde{\alpha}_{1}\right|_{2,-\mu, \Omega}^{2}+c(1 / \varepsilon)|v|_{\infty, \Omega}^{2}\left|\bar{\alpha}_{1}\right|_{2,-\mu, \Omega}^{2},
$$

$$
\varepsilon\left(\left|\widetilde{\alpha}_{1}\right|_{2,-\mu, \Omega}^{2}+\left|\widetilde{\alpha}_{3}\right|_{2,-\mu, \Omega}^{2}\right)+c(1 / \varepsilon)\left|v_{, x}\right|_{\infty, \Omega}^{2}\left|\bar{\alpha}_{1}\right|_{2,-\mu, \Omega}^{2} .
$$

Finally, the last term on the r.h.s. of (4.38) is estimated by

$$
\varepsilon\left|\bar{\alpha}_{1}\right|_{2,-(1+\mu), \Omega}^{2}+c(1 / \varepsilon)\left|\widetilde{\alpha}_{1}\right|_{2,-(1+\mu), \Omega}^{2} .
$$


In view of the above considerations, for $\varepsilon$ sufficiently small we obtain from (4.38) the inequality

$$
\begin{aligned}
\frac{1}{2} \frac{d}{d t}\left|\bar{\alpha}_{1}\right|_{2,-\mu, \Omega}^{2}+ & \nu(1-\varepsilon / 2)\left|\bar{\alpha}_{1, x}\right|_{2,-\mu, \Omega}^{2}+\nu\left(1-2 \mu^{2} / \varepsilon-\varepsilon_{0}\right)\left|\bar{\alpha}_{1}\right|_{2,-(1+\mu), \Omega}^{2} \\
\leq & c\left(1 / \varepsilon_{0}\right)|v|_{\infty, \Omega}^{2}\left|\bar{\alpha}_{1}\right|_{2,-\mu, \Omega}^{2}+c\left(1 / \varepsilon_{1}\right)\left|v v_{, x}\right|_{\infty, \Omega}^{2}\left|\bar{\alpha}_{1}\right|_{2,-\mu, \Omega}^{2} \\
& +\varepsilon_{1}\left(\left|\bar{\alpha}_{1}\right|_{2,-\mu, \Omega}^{2}+\left|\bar{\alpha}_{3}\right|_{2,-\mu, \Omega}^{2}\right)+c\left(1 / \varepsilon_{1}\right) \int_{\Omega}\left|\alpha_{2} / r\right|^{2}|h|^{2} r^{-2 \mu} d x \\
& +c|h|_{2,-1, \Omega}^{2}+\varepsilon_{2}\left(\left\|\bar{\alpha}_{1}\right\|_{2,2,1-\mu, \Omega}^{2}+\left\|\bar{\alpha}_{3}\right\|_{2,2,1-\mu, \Omega}^{2}\right) \\
& +c\left(1 / \varepsilon_{2}\right)\left(\left\|\bar{\alpha}_{1}\right\|_{1, \Omega}^{2}+\left\|\bar{\alpha}_{3}\right\|_{1, \Omega}^{2}\right)+c\left|F_{1}\right|_{2,-\mu, \Omega}^{2}+c\left|\nabla \widetilde{\alpha}_{1}\right|_{2,-\mu, \Omega}^{2} \\
& +\varepsilon_{1}\left(\left|\widetilde{\alpha}_{1}\right|_{2,-\mu, \Omega}^{2}+\left|\widetilde{\alpha}_{3}\right|_{2,-\mu, \Omega}^{2}\right)+c\left(1 / \varepsilon_{0}\right)\left|\widetilde{\alpha}_{1}\right|_{2,-(1+\mu), \Omega}^{2} .
\end{aligned}
$$

From (4.32) we have

$$
\begin{aligned}
\left|\widetilde{\alpha}_{1}\right|_{2, \Omega}^{2}+\left|\widetilde{\alpha}_{3}\right|_{2, \Omega}^{2}+\left\|\widetilde{\alpha}_{1}\right\|_{2,2,1-\mu, \Omega^{t}}^{2}+\left\|\widetilde{\alpha}_{3}\right\|_{2,2,1-\mu, \Omega^{t}}^{2} & \\
& \leq c\left(\|w\|_{2,1-\mu, \Omega^{t}}^{2}+\|h\|_{2,-1, \Omega^{t}}^{2}\right) .
\end{aligned}
$$

Moreover, (4.36) can be written in the form

$$
\begin{aligned}
& \left|\bar{\alpha}_{1}\right|_{2, \Omega}^{2}+\left|\bar{\alpha}_{3}\right|_{2, \Omega}^{2}+\nu\left(\left|\bar{\alpha}_{1, x}\right|_{2, \Omega}^{2}+\left|\bar{\alpha}_{3, x}\right|_{2, \Omega}^{2}\right) \\
& \quad \leq c \exp \left(c\|v\|_{A_{1-\mu}\left(\Omega^{t}\right)}^{2}\right)\left[\left|\alpha_{2}\right|_{10 / 3, \Omega^{t}}^{2}\|h\|_{2,-1, \Omega^{t}}^{2}\right. \\
& \left.\quad+\left(\|h\|_{2,-1, \Omega^{t}}^{2}+\|w\|_{2,1-\mu, \Omega^{t}}^{2}\right)\left(1+\|v\|_{A_{1-\mu}\left(\Omega^{t}\right)}^{2}\right)+\left|F^{\prime}\right|_{2, \Omega^{t}}^{2}+\left|\alpha^{\prime}(0)\right|_{2, \Omega}^{2}\right] .
\end{aligned}
$$

Using (4.43) in (4.12) yields

$$
\begin{aligned}
\left\|\alpha^{\prime}\right\|_{2,2,1-\mu, \Omega^{t}} \leq & \varphi\left(\|v\|_{A_{1-\mu}\left(\Omega^{t}\right)}\right)\left[\left|\alpha_{2} / r\right|_{10 / 3, \Omega^{t}}\|h\|_{2,-1, \Omega^{t}}\right. \\
& \left.+\|h\|_{2,-1, \Omega^{t}}+\|w\|_{2,1-\mu, \Omega^{t}}+\left|F^{\prime}\right|_{2, \Omega^{t}}+\left\|\alpha^{\prime}(0)\right\|_{1,2,1-\mu, \Omega}\right] \\
& +c\left|\alpha_{1}\right|_{2,-(1+\mu), \Omega^{t}}
\end{aligned}
$$

Integrating (4.41) with respect to time yields

$$
\begin{aligned}
\frac{1}{2}\left|\bar{\alpha}_{1}\right|_{2,-\mu, \Omega}^{2} & +\nu(1-\varepsilon / 2)\left|\bar{\alpha}_{1, x}\right|_{2,-\mu, \Omega^{t}}^{2} \\
& +\nu\left(1-2 \mu^{2} / \varepsilon-\varepsilon_{0}\right)\left|\bar{\alpha}_{1}\right|_{2,-(1+\mu), \Omega^{t}}^{2} \\
\leq & c \exp \left(c\left(1 / \varepsilon_{0}\right)\|v\|_{A_{1-\mu}\left(\Omega^{t}\right)}\right)\left[\left|\bar{\alpha}_{1}\right|_{2,-\mu, \Omega^{t}}^{2}+\left|\bar{\alpha}_{3}\right|_{2,-\mu, \Omega^{t}}^{2}\right. \\
& +\left|\alpha_{2} / r\right|_{10 / 3, \Omega^{t}}^{2}\|h\|_{2,-1, \Omega^{t}}^{2}+\|h\|_{2,-1, \Omega^{t}}^{2} \\
& +\varepsilon_{2}\left(\left\|\bar{\alpha}_{1}\right\|_{2,2,1-\mu, \Omega^{t}}^{2}+\left\|\bar{\alpha}_{3}\right\|_{2,2,1-\mu, \Omega^{t}}^{2}\right)+c\left(1 / \varepsilon_{2}\right)\left(\left\|\bar{\alpha}_{1}\right\|_{1, \Omega}^{2}+\left\|\bar{\alpha}_{3}\right\|_{1, \Omega}^{2}\right) \\
& +\left|F_{1}\right|_{2,-\mu, \Omega^{t}}^{2}+\left|\nabla \widetilde{\alpha}_{1}\right|_{2,-\mu, \Omega^{t}}^{2}+\left|\widetilde{\alpha}_{1}\right|_{2,-\mu, \Omega^{t}}^{2}+\left|\widetilde{\alpha}_{3}\right|_{2,-\mu, \Omega^{t}}^{2} \\
& \left.+\frac{1}{2}\left|\bar{\alpha}_{1}(0)\right|_{2,-\mu, \Omega}^{2}\right] .
\end{aligned}
$$


Using (4.42) and (4.44) in (4.45) we have

$$
\begin{aligned}
& \frac{1}{2}\left|\bar{\alpha}_{1}\right|_{2,-\mu, \Omega}^{2}+\nu(1-\varepsilon / 2)\left|\bar{\alpha}_{1, x}\right|_{2,-\mu, \Omega^{t}}^{2}+\nu\left(1-2 \mu^{2} / \varepsilon-\varepsilon_{0}\right)\left|\bar{\alpha}_{1}\right|_{2-(1+\mu), \Omega^{t}} \\
& \leq \varphi\left(1 / \varepsilon_{0},\|v\|_{A_{1-\mu}\left(\Omega^{t}\right)}\right)\left[\left|\bar{\alpha}_{1}\right|_{2,-\mu, \Omega^{t}}^{2}+\left|\bar{\alpha}_{3}\right|_{2,-\mu, \Omega^{t}}^{2}\right. \\
& \quad+\left|\alpha_{2} / r\right|_{10 / 3, \Omega^{t}}^{2}\|h\|_{2,-1, \Omega^{t}}^{2}+\|h\|_{2,-1, \Omega^{t}}^{2}+\|w\|_{2,1-\mu, \Omega^{t}}^{2}+\left|F^{\prime}\right|_{2, \Omega^{t}}^{2}+\left|F_{1}\right|_{2,-\mu, \Omega^{t}}^{2} \\
& \left.\quad+\left\|\alpha^{\prime}(0)\right\|_{1,2,1-\mu, \Omega}^{2}+\frac{1}{2}\left|\bar{\alpha}_{1}(0)\right|_{2,-\mu, \Omega}^{2}+\left\|\bar{\alpha}_{1}\right\|_{1, \Omega}^{2}+\left\|\bar{\alpha}_{3}\right\|_{1, \Omega}\right] .
\end{aligned}
$$

Employing (4.43) in (4.46) yields

$$
\begin{aligned}
& \frac{1}{2}\left|\bar{\alpha}_{1}\right|_{2,-\mu, \Omega}^{2}+\nu(1-\varepsilon / 2)\left|\bar{\alpha}_{1, x}\right|_{2,-\mu, \Omega^{t}}^{2}+\nu\left(1-2 \mu^{2} / \varepsilon-\varepsilon_{0}\right)\left|\bar{\alpha}_{1}\right|_{2,-(1+\mu), \Omega^{t}}^{2} \\
& \leq \varphi\left(1 / \varepsilon_{0},\|v\|_{A_{1-\mu}\left(\Omega^{t}\right)}\right)\left[\left|\alpha_{2} / r\right|_{10 / 3, \Omega^{t}}^{2}\|h\|_{2,-1, \Omega^{t}}^{2}+\|h\|_{2,-1, \Omega^{t}}^{2}+\|w\|_{2,1-\mu, \Omega^{t}}^{2}\right. \\
& \left.\quad+\left|F^{\prime}\right|_{2, \Omega^{t}}^{2}+\left|F_{1}\right|_{2,-\mu, \Omega^{t}}^{2}+\left\|\alpha^{\prime}(0)\right\|_{1,2,1-\mu, \Omega}^{2}+\left|\bar{\alpha}_{1}(0)\right|_{2,-\mu, \Omega}\right] .
\end{aligned}
$$

Finally, from (4.47) and (4.35) we obtain (4.37). This concludes the proof.

To obtain estimates for solutions of problem (1.8) we write it in the form

$$
\begin{aligned}
& \alpha_{2, t}+v \cdot \nabla \alpha_{2}-\frac{1}{r} h_{\varphi} \alpha_{2}-\frac{v_{r}}{r} \alpha_{2}+\frac{\nu \alpha_{2}}{r^{2}}-\nu \Delta \alpha_{2} \\
&= \frac{2 \nu}{r^{2}}\left(\frac{1}{r} h_{z, \varphi}-h_{\varphi, z}\right)-\frac{1}{r}\left(w_{, z} h_{r}-w_{, r} h_{z}+\frac{w}{r} h_{z}\right)+\frac{2}{r} w v_{\varphi, z}+F_{2}, \\
&\left.\alpha_{2}\right|_{S}=2(k-\gamma /(2 \nu)) v \cdot \bar{\tau}_{2}, \\
&\left.\alpha_{2}\right|_{t=0}=\alpha_{2}(0),
\end{aligned}
$$

where $k$ and $\gamma$ are defined in Section 1 .

For solutions of (4.48) we have

Lemma 4.6. Assume that $v \in A_{1-\mu}\left(\Omega^{t}\right), \alpha_{2} / r \in L_{2}\left(0, t ; H^{1}(\Omega)\right) \cap L_{10 / 3}\left(\Omega^{t}\right), \alpha_{2} \in$ $L_{2,-(1+\mu)}\left(\Omega^{t}\right), h \in H_{-1}^{2,1}\left(\Omega^{t}\right), F_{2} \in L_{2,1-\mu}\left(\Omega^{t}\right), w \in L_{\infty}\left(0, t ; H_{0}^{1}(\Omega)\right), v(0) \in W_{2,1-\mu}^{2}(\Omega) \cap$ $H^{1}(\Omega), \alpha_{2}(0) \in W_{2,1-\mu}^{1}(\Omega), t \leq T, \mu \in(1 / 2,1)$. Then solutions of problem (4.48) satisfy, for any $\varepsilon_{1}, \varepsilon_{2}>0$,

$$
\begin{aligned}
\left\|\alpha_{2}\right\|_{2,2,1-\mu, \Omega^{t}} \leq & {\left[\varepsilon_{1}\|v\|_{A_{1-\mu}\left(\Omega^{t}\right)}\right.} \\
& \left.\left.+c \varepsilon_{1}^{-3 / 4} \underset{t}{\sup }|v|_{2, \Omega}+|v|_{2, \Omega^{t}}\right)+\|v(0)\|_{2,2,1-\mu, \Omega}\right] \\
& \cdot\left[\left|\alpha_{2} / r\right|_{10 / 3, \Omega^{t}}+\left(\int_{0}^{t}\left|\nabla\left(\alpha_{2}\left(t^{\prime}\right) / r\right)\right|_{2, \Omega}^{2} d t^{\prime}\right)^{1 / 2}\right] \\
& +c\left(1+\left|\alpha_{2} / r\right|_{10 / 3, \Omega^{t}}+\sup _{t}\|w\|_{1,0, \Omega}\right)\|h\|_{2,-1, \Omega^{t}} \\
& +\left[\sup _{t}\|w\|_{1,0, \Omega}\|v\|_{A_{1-\mu}\left(\Omega^{t}\right)}+\left|\alpha_{2}\right|_{2,-(1+\mu), \Omega^{t}}+\left|F_{2}\right|_{2,1-\mu, \Omega^{t}}\right. \\
& \left.+\delta_{0}\left(\varepsilon_{2}\|v\|_{A_{1-\mu}\left(\Omega^{t}\right)}+\left(1 / \varepsilon_{2}\right)|v|_{2, \Omega^{t}}\right)+\left\|\alpha_{2}(0)\right\|_{1,2,1-\mu, \Omega}\right],
\end{aligned}
$$

where $\delta_{0}^{1}=\|k-\gamma /(2 \nu)\|_{3 / 2, S}$ and $\delta_{0}^{1} \leq c \delta_{0}$. 
Proof. Applying [zaj 1] to (4.48) yields

$$
\begin{aligned}
\left\|\alpha_{2}\right\|_{2,2,1-\mu, \Omega^{t}} & \leq c\left[\left|v \cdot \nabla \alpha_{2}\right|_{2,1-\mu, \Omega^{t}}+\left|\frac{1}{r} h_{\varphi} \alpha_{2}\right|_{2,1-\mu, \Omega^{t}}\right. \\
+ & \left|\frac{v_{r}}{r} \alpha_{2}\right|_{2,1-\mu, \Omega^{t}}+\left|\alpha_{2}\right|_{2,-(1+\mu), \Omega^{t}}+\left|\frac{1}{r^{2}}\left(\frac{1}{r} h_{z, \varphi}-h_{\varphi, z}\right)\right|_{2,1-\mu, \Omega^{t}} \\
& +\left|\frac{1}{r}\left(w_{, z} h_{r}-w_{, r} h_{z}+\frac{w}{r} h_{z}\right)\right|_{2,1-\mu, \Omega^{t}}+\left|\frac{w}{r} v_{\varphi, z}\right|_{2,1-\mu, \Omega^{t}} \\
& \left.+\left|F_{2}\right|_{2,1-\mu, \Omega^{t}}+\left\|(k-\gamma /(2 \nu)) v \cdot \bar{\tau}_{2}\right\|_{3 / 2,1-\mu, S^{t}}+\left\|\alpha_{2}(0)\right\|_{1,2,1-\mu, \Omega}\right] .
\end{aligned}
$$

Now we examine the particular terms on the r.h.s. of (4.50). The first and third terms are estimated by

$$
\begin{aligned}
\mid v \cdot \nabla\left(\frac{\alpha_{2}}{r} r\right) & \left.\right|_{2,1-\mu, \Omega^{t}}+\left|v_{r} \frac{\alpha_{2}}{r}\right|_{2,1-\mu, \Omega^{t}} \\
& \leq c\left(\left|v \cdot \nabla \frac{\alpha_{2}}{r}\right|_{2,1-\mu, \Omega^{t}}+\left|v_{r} \frac{\alpha_{2}}{r}\right|_{2,1-\mu, \Omega^{t}}\right) \\
& \leq c \sup _{t}\left|v r^{1-\mu}\right|_{\infty, \Omega}\left(\int_{0}^{t}\left|\nabla\left(\alpha_{2} / r\right)\right|_{2, \Omega}^{2} d t^{\prime}\right)^{1 / 2}+c\left|v_{r}\right|_{5,1-\mu, \Omega^{t}}\left|\alpha_{2} / r\right|_{10 / 3, \Omega^{t}} \\
& \equiv c\left(I_{1}+I_{2}\right)
\end{aligned}
$$

where the first factor in $I_{1}$ is estimated by

$$
\begin{aligned}
\sup _{t}\left(\varepsilon_{1}^{\prime}\|v\|_{2,2,1-\mu, \Omega}+c\left(1 / \varepsilon_{1}^{\prime}\right)|v|_{2, \Omega}\right) & \\
& \leq \varepsilon_{1}^{\prime}\|v\|_{A_{1-\mu}\left(\Omega^{t}\right)}+c \varepsilon_{1}^{\prime-3 / 4} \sup _{t}|v|_{2, \Omega}+\varepsilon_{1}^{\prime}\|v(0)\|_{2,2,1-\mu, \Omega},
\end{aligned}
$$

and the first factor in $I_{2}$ by

$$
\varepsilon_{2}^{\prime}\|v\|_{2,2,1-\mu, \Omega^{t}}+c \varepsilon_{2}^{\prime-3 / 4}|v|_{2, \Omega^{t}} .
$$

Summarizing, we dominate the first and third terms on the r.h.s. of (4.50) by

$$
\begin{aligned}
{\left[\varepsilon_{1}\|v\|_{A_{1-\mu}\left(\Omega^{t}\right)}+c\left(1 / \varepsilon_{1}\right)\left(\sup _{t}|v|_{2, \Omega}+|v|_{2, \Omega^{t}}\right)+c\|v(0)\|_{2,2,1-\mu, \Omega}\right] } & \\
\cdot & {\left[\left(\int_{0}^{t}\left|\nabla\left(\alpha_{2} / r\right)\right|_{2, \Omega}^{2} d t^{\prime}\right)^{1 / 2}+\left|\alpha_{2} / r\right|_{10 / 3, \Omega^{t}}\right] . }
\end{aligned}
$$

We bound the second term by

$$
c\left|\alpha_{2} / r\right|_{10 / 3, \Omega^{t}}\|h\|_{2,-1, \Omega^{t}}
$$

and the sixth by

$$
\left\|\nabla w \frac{h}{r}|+| \frac{w}{r} \frac{h}{r}\right\|_{2,1-\mu, \Omega^{t}} \leq c \sup _{t}\|w\|_{1,0, \Omega}\left(\int_{0}^{t}\left|h r^{-\mu}\right|_{\infty, \Omega}^{2} d t^{\prime}\right)^{1 / 2} \equiv I_{3} .
$$


Employing (2.8) we have

$$
I_{3} \leq c \sup _{t}\|w\|_{1,0, \Omega}\left(\int_{0}^{t}\left\|h\left(t^{\prime}\right)\right\|_{2,1 / 2-\mu, \Omega}^{2} d t^{\prime}\right)^{1 / 2} \leq c \sup _{t}\|w\|_{1,0, \Omega}\|h\|_{2,-1, \Omega^{t}} .
$$

The seventh term is dominated by

$$
\begin{aligned}
& \left|\frac{w}{r} v_{\varphi, z}\right|_{2,1-\mu, \Omega^{t}} \leq \sup _{t}\left|\frac{w}{r}\right|_{2, \Omega}\left(\int_{0}^{t}\left|v_{\varphi, z} r^{1-\mu}\right|_{\infty, \Omega}^{2} d t^{\prime}\right)^{1 / 2} \\
& \quad \leq \sup _{t}\|w\|_{1,0, \Omega}\left(\int_{0}^{t}\left\|v_{\varphi, z}\left(t^{\prime}\right)\right\|_{2,2,1-\mu, \Omega}^{2} d t^{\prime}\right)^{1 / 2} \leq c \sup _{t}\|w\|_{1,0, \Omega}\|v\|_{A_{1-\mu}\left(\Omega^{t}\right)} .
\end{aligned}
$$

Finally, the ninth term is estimated as follows:

$$
\begin{aligned}
\|(k-\gamma /(2 \nu)) & v \cdot \bar{\tau}_{2} \|_{3 / 2,2,1-\mu, S^{t}} \\
& \leq c\left[\int_{0}^{t}\left(\left|\partial_{s}^{3 / 2} v\left(t^{\prime}\right)\right|_{2,1-\mu, S}^{2}+\left|\partial_{t^{\prime}}^{3 / 4} v\left(t^{\prime}\right)\right|_{2,1-\mu, S}^{2}+\left|v\left(t^{\prime}\right)\right|_{2,1-\mu, S}^{2}\right) d t^{\prime}\right]^{1 / 2} \delta_{0} \\
& \leq\left(\int_{0}^{t}\left[\varepsilon_{2}\left(\left|v_{, x x x}\right|_{2,1-\mu, \Omega}^{2}+\left|v_{, t x}\right|_{2,1-\mu, \Omega}^{2}\right)+c\left(1 / \varepsilon_{2}\right)|v|_{2, \Omega}^{2}\right] d t^{\prime}\right)^{1 / 2} \delta_{0} \\
& \leq\left(\varepsilon_{2}\|v\|_{A_{1-\mu}\left(\Omega^{t}\right)}+c\left(1 / \varepsilon_{2}\right)|v|_{2, \Omega^{t}}\right) \delta_{0} .
\end{aligned}
$$

Using the above estimates in (4.50) implies (4.49). This ends the proof.

Now we shall estimate the expression

$$
I_{1}=\left(\int_{0}^{t}\left|v_{\varphi, z}\left(t^{\prime}\right)\right|_{4,-3 / 4-\varepsilon, \Omega}^{2} d t^{\prime}\right)^{1 / 2}, \quad \varepsilon>0,
$$

which appears on the r.h.s. of (4.1). Using (1.6) we have

$$
v_{\varphi, z}=-\alpha_{1}+\frac{1}{r} h_{z}
$$

In view of (4.51) we get

$$
I_{1} \leq c\left(\int_{0}^{t}\left|\alpha_{1}\left(t^{\prime}\right)\right|_{4,-3 / 4-\varepsilon, \Omega}^{2} d t^{\prime}\right)^{1 / 2}+c\left(\int_{0}^{t}\left\|h\left(t^{\prime}\right)\right\|_{2,-1, \Omega}^{2} d t^{\prime}\right)^{1 / 2}
$$

for any small positive number $\varepsilon$. To estimate the first term on the r.h.s. of (4.52) we need (4.47) in the form

$$
\begin{aligned}
\left|\bar{\alpha}_{1, x}\right|_{2,-\mu, \Omega^{t}}^{2}+\left|\bar{\alpha}_{1}\right|_{2,-(1+\mu), \Omega^{t}}^{2} \leq & \varphi\left(\|v\|_{A_{1-\mu}\left(\Omega^{t}\right)}\right)\left[\left|\alpha_{2} / r\right|_{10 / 3, \Omega^{t}}^{2}\|h\|_{2,-1, \Omega^{t}}^{2}\right. \\
& +\|h\|_{2,-1, \Omega^{t}}^{2}+\|w\|_{2,1-\mu, \Omega^{t}}^{2}+\left|F^{\prime}\right|_{2, \Omega^{t}}^{2}+\left|F_{1}\right|_{2,-\mu, \Omega^{t}}^{2} \\
& \left.+\left\|\alpha^{\prime}(0)\right\|_{1,2,1-\mu, \Omega}^{2}+\left|\bar{\alpha}_{1}(0)\right|_{2,-\mu, \Omega}^{2}\right]
\end{aligned}
$$

where we used the fact that $\mu \in(0,1), \varepsilon \in(0,2), \varepsilon_{0}<1-2 \mu^{2} / \varepsilon$.

Since $\alpha_{1}=\bar{\alpha}_{1}+\widetilde{\alpha}_{1}$ we need (4.35),

$$
\left|\widetilde{\alpha}_{1}\right|_{2,-(1+\mu), \Omega^{t}} \leq c\left(\|w\|_{2,1-\mu, \Omega^{t}}+\|h\|_{2,-1, \Omega^{t}}\right) .
$$


Moreover, (4.32) and the Hardy inequality imply

$$
\left|\widetilde{\alpha}_{1, x}\right|_{2,-\mu, \Omega^{t}} \leq c\left(\|w\|_{2,1-\mu, \Omega^{t}}+\|h\|_{2,-1, \Omega^{t}}\right) .
$$

Summarizing we have

$$
\begin{aligned}
\left(\int_{0}^{t}\left\|\alpha_{1}\right\|_{1,-\mu, \Omega}^{2} d t^{\prime}\right)^{1 / 2} \leq & \varphi\left(\|v\|_{A_{1-\mu}\left(\Omega^{t}\right)}\right)\left[\left|\alpha_{2} / r\right|_{10 / 3, \Omega^{t}}\|h\|_{2,-1, \Omega^{t}}\right. \\
& +\|h\|_{2,-1, \Omega^{t}}+\|w\|_{2,1-\mu, \Omega^{t}}+\left|F^{\prime}\right|_{2, \Omega^{t}}+\left|F_{1}\right|_{2,-\mu, \Omega^{t}} \\
& \left.+\left\|\alpha^{\prime}(0)\right\|_{1,2,1-\mu, \Omega}+\left|\alpha_{1}^{\prime}(0)\right|_{2,-\mu, \Omega}\right],
\end{aligned}
$$

for any $\mu \in(1 / 2,1)$.

In view of (2.8) the first term on the r.h.s of (4.52) is bounded by

$$
c\left(\int_{0}^{t}\left\|\alpha_{1}\left(t^{\prime}\right)\right\|_{1,-1 / 2-\varepsilon}^{2} d t^{\prime}\right)^{1 / 2} \equiv I_{2},
$$

and to estimate $I_{2}$ we use (4.56) with $\mu=1 / 2+\varepsilon, \varepsilon>0$ a small number.

Summarizing the above considerations we obtain

LEMma 4.7. Assume that $\alpha_{2} / r \in L_{10 / 3}\left(\Omega^{t}\right), v \in A_{1-\mu}\left(\Omega^{t}\right), h \in H_{-1}^{2,1}\left(\Omega^{t}\right), w \in H_{1-\mu}^{2,1}\left(\Omega^{t}\right)$, $F^{\prime} \in L_{2}\left(\Omega^{t}\right), F_{1} \in L_{2,-\mu}\left(\Omega^{t}\right), \alpha^{\prime}(0) \in W_{2,1-\mu}^{1}(\Omega), \alpha_{1}(0) \in L_{2,-\mu}(\Omega)$, where $\mu=1 / 2+\varepsilon$ and $\varepsilon$ is an arbitrary small number. Then

$$
\begin{gathered}
\left(\int_{0}^{t}\left|v_{\varphi, z}\left(t^{\prime}\right)\right|_{4,-3 / 4-\varepsilon}^{2} d t^{\prime}\right)^{1 / 2} \leq \varphi\left(\|v\|_{A_{1-\mu}\left(\Omega^{t}\right)}\right) \\
\cdot\left[\left|\alpha_{2} / r\right|_{10 / 3, \Omega^{t}}\|h\|_{2,-1, \Omega^{t}}+\|h\|_{2,-1, \Omega^{t}}+\|w\|_{2,1-\mu, \Omega^{t}}+\left|F^{\prime}\right|_{2, \Omega^{t}}\right. \\
\left.+\left|F_{1}\right|_{2,-\mu, \Omega^{t}}+\left\|\alpha^{\prime}(0)\right\|_{1,2,1-\mu, \Omega}+\left|\alpha_{1}(0)\right|_{2,-\mu, \Omega}\right] .
\end{gathered}
$$




\section{Estimates for the azimuthal coordinate of velocity}

In this section we obtain estimates for solutions of problem (1.5).

Lemma 5.1. Assume that $f_{\varphi} \in L_{2,1-\mu}\left(\Omega^{T}\right), w(0) \in H_{1-\mu}^{1}(\Omega), w \in L_{2,-(1+\mu)}\left(\Omega^{T}\right), g \in$ $L_{2,-1}\left(\Omega^{T}\right), h(0) \in H_{-1}^{1}(\Omega), \mu>1 / 2$. Then

$$
\begin{aligned}
\|w\|_{2,1-\mu, \Omega^{t}} \leq & \varphi\left(\|v\|_{A_{1-\mu}\left(\Omega^{t}\right)}\right)|w|_{2,-(1+\mu), \Omega^{t}}+\varphi\left(t^{1 / 2}\|v\|_{A_{1-\mu}\left(\Omega^{t}\right)}\right) \\
& \cdot\left[|h(0)|_{2, \Omega}+|g|_{2, \Omega^{t}}\right]+c\left[|g|_{2,-1, \Omega^{t}}+\|h(0)\|_{1,-1, \Omega}+\|w(0)\|_{1,1-\mu, \Omega}\right. \\
& \left.+\left|f_{\varphi}\right|_{2,1-\mu, \Omega^{t}}\right],
\end{aligned}
$$

where $\varphi$ is an increasing positive function, $t \leq T$.

Proof. Applying [zaj 1] to problem (1.5) we obtain

$$
\begin{aligned}
& \|w\|_{2,1-\mu, \Omega^{t}} \leq c\left(|v \cdot \nabla w|_{2,1-\mu, \Omega^{t}}+\left|\frac{v_{r} w}{r}\right|_{2,1-\mu, \Omega^{t}}\right. \\
& +|q|_{2,-\mu, \Omega^{t}}+|h|_{2,-(1+\mu), \Omega^{t}}+|w|_{2,-(1+\mu), \Omega^{t}}+\left|f_{\varphi}\right|_{2,1-\mu, \Omega^{t}} \\
& \left.+\left\|\frac{1}{r} w\right\|_{1 / 2,1-\mu, S^{T}}+\|w(0)\|_{1,1-\mu, \Omega}\right) .
\end{aligned}
$$

In view of [zaj 3] we estimate the first term on the r.h.s. of (5.2) by

$$
\begin{aligned}
|v \cdot \nabla w|_{2,1-\mu, \Omega^{t}} & \leq|v|_{2 p_{1}, \Omega^{t}}|\nabla w|_{2 p_{2}, 1-\mu, \Omega^{t}} \\
& \leq \varepsilon\|w\|_{2,1-\mu, \Omega^{t}}+c(1 / \varepsilon) \varphi\left(\|v\|_{2,2,1-\mu, \Omega^{T}}\right)|w|_{2,-(1+\mu), \Omega^{t}}
\end{aligned}
$$

whenever $1 / p_{1}+1 / p_{2}=1$; here we used the imbeddings $W_{2,1-\mu}^{2,1}\left(\Omega^{T}\right) \subset L_{2 p_{1}}\left(\Omega^{T}\right)$, $\nabla H_{1-\mu}^{2,1}\left(\Omega^{t}\right) \subset L_{2 p_{2}, 1-\mu}\left(\Omega^{T}\right)$, which hold together for $\mu>1 / 2$.

The second term on the r.h.s. of (5.2) is dealt with as follows:

$$
\begin{aligned}
\left|\frac{v_{r} w}{r}\right|_{2,1-\mu, \Omega^{t}} & \leq\left|v_{r}\right|_{2 p_{1}, \Omega^{t}}\left|\frac{w}{r}\right|_{2 p_{2}, 1-\mu, \Omega^{t}}=\left|v_{r}\right|_{2 p_{1}, \Omega^{t}}|w|_{2 p_{2},-\mu, \Omega^{t}} \\
& \leq \varepsilon\|w\|_{2,1-\mu, \Omega^{t}}+c(1 / \varepsilon) \varphi\left(\|v\|_{2,2,1-\mu, \Omega^{t}}\right)|w|_{2,-(1+\mu), \Omega^{t}}
\end{aligned}
$$

whenever $1 / p_{1}+1 / p_{2}=1$; here we used the imbeddings $W_{2,1-\mu}^{2,1}\left(\Omega^{T}\right) \subset L_{2 p_{1}}\left(\Omega^{t}\right)$ and $H_{1-\mu}^{2,1}\left(\Omega^{T}\right) \subset L_{2 p_{2},-\mu}\left(\Omega^{T}\right)$ which hold together for $\mu>1 / 2$.

To estimate the boundary term in the r.h.s. of (5.2) we use

$$
\left\|\frac{1}{r} w\right\|_{1 / 2,1-\mu, S^{t}}=\|w\|_{1 / 2,-\mu, S^{t}} \leq \varepsilon\|w\|_{2,1-\mu, \Omega^{t}}+c(1 / \varepsilon)|w|_{2,-(1+\mu), \Omega^{t}}
$$

for $\mu>1 / 2$.

Using the above estimates with sufficiently small $\varepsilon$ and (4.8) in (5.2) we obtain (5.1). This concludes the proof. 
To estimate the norm $|w|_{2,-(1+\mu), \Omega^{t}}$ in $(5.1)$ we need

Lemma 5.2. Assume that $\mu \in(1 / 2,1), q \in L_{2}\left(0, T ; L_{2,-\mu}(\Omega)\right), h \in L_{2}\left(0, T ; L_{2,-(1+\mu)}(\Omega)\right)$, $f_{\varphi} \in L_{2}\left(0, T ; L_{2,-\mu+1}(\Omega)\right), w(0) \in L_{2,-\mu}(\Omega), v_{r} \in L_{2}\left(0, T ; W_{2,1-\mu}^{2}(\Omega)\right)$. Assume that either $\gamma / \nu-a_{1} /\left.r\right|_{S} \geq 0$ or $\left|a_{1}\right| \leq c r$, for $r$ close to 0 . Then

$$
\begin{aligned}
|w|_{2,-\mu, \Omega}^{2}+ & \nu\left(1-\varepsilon_{0} / 2-\varepsilon_{1}\right)|\nabla w|_{2,-\mu, \Omega^{t}}^{2} \\
& +\nu\left(1-2 \mu^{2} / \varepsilon_{0}-\varepsilon_{2}-\varepsilon_{3}\right)|w|_{2,-(1+\mu), \Omega^{t}}^{2}+\gamma|w|_{2,-\mu, S^{t}}^{2} \\
\leq & c\left(1 / \varepsilon_{3}\right) \exp \left[c\left(1 / \varepsilon_{1}, 1 / \varepsilon_{2}\right)\left(t+\int_{0}^{t}\left\|v_{r}\right\|_{2,2,1-\mu, \Omega}^{2} d t^{\prime}\right)\right] \\
& \cdot\left[|q|_{2,-\mu, \Omega^{t}}^{2}+|h|_{2,-(1+\mu), \Omega^{t}}^{2}+\left|f_{\varphi}\right|_{2,-\mu+1, \Omega^{t}}^{2}+|w(0)|_{2,-\mu, \Omega}^{2}\right], \quad t \leq T,
\end{aligned}
$$

for any $\varepsilon_{i}, i=0, \ldots, 3$, such that $1-\varepsilon_{0} / 2-\varepsilon_{1}>0,1-2 \mu^{2} / \varepsilon_{0}-\varepsilon_{2}-\varepsilon_{3}>0$.

Proof. Multiplying (1.5) 1 by $w r^{-2 \mu}$ and integrating over $\Omega$ we get

$$
\begin{aligned}
\frac{1}{2} \frac{d}{d t}|w|_{2,-\mu, \Omega}^{2}-\nu \int_{\Omega} \Delta w w r^{-2 \mu} d x+\nu|w|_{2,-(1+\mu), \Omega}^{2} & \\
= & -\int_{\Omega}\left(v \cdot \nabla w+\frac{v_{r}}{r} w\right) w r^{-2 \mu} d x+\int_{\Omega} \frac{1}{r} q w r^{-2 \mu} d x+2 \nu \int_{\Omega} \frac{h_{r}}{r^{2}} w r^{-2 \mu} d x \\
& +\int_{\Omega} f_{\varphi} w r^{-2 \mu} d x .
\end{aligned}
$$

The second term on the l.h.s. equals

$$
I_{1} \equiv \nu \int_{S}\left(\frac{\gamma}{\nu}-\frac{a_{1}}{r}\right) w^{2} r^{-2 \mu} d S+\nu \int_{\Omega}|\nabla w|^{2} r^{-2 \mu} d x-2 \mu \nu \int_{\Omega} \nabla w w r^{-2 \mu-1} \nabla r d x .
$$

To estimate the boundary term in $I_{1}$ we consider two cases. For $\gamma / \nu-a_{1} /\left.r\right|_{S} \geq 0$ the term can be omitted.

For $\gamma / \nu-a_{1} /\left.r\right|_{S}<0$ we use the fact that $\left|a_{1}\right| \leq c r, r \leq r_{0}$. Then

$$
\begin{aligned}
& \left|\int_{S \cap\left\{x: r \leq r_{0}\right\}} \frac{a_{1}}{r} r^{-2 \mu} w^{2} d S\right| \\
& \quad \leq c\left(r_{0}\right) \int_{S \cap\left\{x: r \leq r_{0}\right\}} w^{2} r^{-2 \mu} d S \leq \varepsilon_{1}\left|w_{, x}\right|_{2,-\mu, \Omega}^{2}+c\left(1 / \varepsilon_{1}, r_{0}\right)|w|_{2,-\mu, \Omega}^{2}
\end{aligned}
$$

and

$$
\begin{aligned}
& \left|\int_{S \cap\left\{x: r \geq r_{0}\right\}} \frac{a_{1}}{r} r^{-2 \mu} w^{2} d S\right| \\
& \quad \leq c\left(1 / r_{0}\right)\left|a_{1}\right|_{\infty, S}|w|_{2,-\mu, S}^{2} \leq \varepsilon_{1}\left|w_{, x}\right|_{2,-\mu, \Omega}^{2}+c\left(1 / \varepsilon_{1}, 1 / r_{0},\left|a_{1}\right|_{\infty, S}\right)|w|_{2,-\mu, \Omega}^{2} .
\end{aligned}
$$

We bound the last term in $I_{1}$ by

$$
\nu \frac{\varepsilon_{0}}{2}|\nabla w|_{2,-\mu, \Omega}^{2}+\nu \frac{2 \mu^{2}}{\varepsilon_{0}}|w|_{2,-(1+\mu), \Omega}^{2} .
$$


The first term on the r.h.s. of (5.4) takes the form

$$
\begin{aligned}
& -\int_{\Omega}\left(v \cdot \nabla w w+\frac{v_{r}}{r} w^{2}\right) r^{-2 \mu} d x=-\int_{\Omega}\left(\frac{1}{2} v \cdot \nabla w^{2}+\frac{v_{r}}{r} w^{2}\right) r^{-2 \mu} d x \\
& =-\int_{\Omega}\left[\frac{1}{2} v \cdot \nabla\left(w^{2} r^{-2 \mu}\right)+(1+\mu) \frac{v_{r}}{r} w^{2} r^{-2 \mu}\right] d x=-(1+\mu) \int_{\Omega} \frac{v_{r}}{r} w^{2} r^{-2 \mu} d x \\
& \equiv I_{2} .
\end{aligned}
$$

Hence

$$
\left|I_{2}\right| \leq \varepsilon_{2}|w|_{2,-(1+\mu), \Omega}^{2}+c\left(1 / \varepsilon_{2}\right)\left|v_{r}\right|_{\infty, \Omega}^{2}|w|_{2,-\mu, \Omega}^{2}
$$

Using [zaj 3] we have

$$
\left|v_{r}\right|_{\infty, \Omega} \leq c\left\|v_{r}\right\|_{2,2,1-\mu, \Omega}
$$

for $\mu>1 / 2$.

We apply the above considerations in (5.4) and in addition use the Hölder and Young inequalities to estimate the last three terms on the r.h.s. of (5.4). Thus we obtain

$$
\begin{aligned}
\frac{1}{2} \frac{d}{d t}|w|_{2,-\mu, \Omega}^{2}+\nu\left(1-\varepsilon_{0} / 2\right. & \left.-\varepsilon_{1}\right)|\nabla w|_{2,-\mu, \Omega}^{2} \\
& +\nu\left(1-2 \mu^{2} / \varepsilon_{0}-\varepsilon_{2}-\varepsilon_{3}\right)|w|_{2,-(1+\mu), \Omega}^{2} \\
\leq & c\left(1 / \varepsilon_{1}, 1 / \varepsilon_{2}\right)\left(1+\| v_{r}||_{2,2,1-\mu, \Omega}^{2}\right)|w|_{2,-\mu, \Omega}^{2} \\
& +c\left(1 / \varepsilon_{3}\right)\left(|q|_{2,-\mu, \Omega}^{2}+|h|_{2,-(1+\mu), \Omega}^{2}+\left|f_{\varphi}\right|_{2,-\mu+1, \Omega}^{2}\right) .
\end{aligned}
$$

Integrating (5.5) with respect to $t$ yields (5.3). This concludes the proof.

Next we need

Lemma 5.3. Assume that $q \in L_{2}\left(\Omega^{t}\right), h \in L_{2,-1}\left(\Omega^{t}\right), f_{\varphi} \in L_{2}\left(\Omega^{t}\right), a_{1} \geq 0, \int_{S^{t}}\left(a_{1} / r\right) w^{2} d S$ $<\infty, w(0) \in L_{2}(\Omega), v_{r} \in L_{2}\left(0, t ; L_{\infty}(\Omega)\right), t \leq T$. Then solutions of (1.5) satisfy

$$
\begin{gathered}
\frac{1}{2}|w(t)|_{2, \Omega}^{2}+\nu\left|w_{, x}\right|_{2, \Omega^{t}}^{2}+\frac{\nu}{2}|w|_{2,-1, \Omega^{t}}^{2}+\gamma|w|_{2, S^{t}}^{2} \\
\leq c \exp \left[c \int_{0}^{t}\left|v_{r}\left(t^{\prime}\right)\right|_{\infty, \Omega}^{2} d t^{\prime}\right]\left[|q|_{2, \Omega^{t}}^{2}+|h|_{2,-1, \Omega^{t}}^{2}+\left|f_{\varphi}\right|_{2, \Omega^{t}}^{2}\right. \\
\left.+\nu \int_{S^{t}} \frac{a_{1}}{r} w^{2} d S+\frac{1}{2}|w(0)|_{2, \Omega}^{2}\right] .
\end{gathered}
$$

Proof. Multiplying (1.5) 1 by $w$ and integrating over $\Omega$ implies

$$
\begin{aligned}
\frac{1}{2} \frac{d}{d t}|w|_{2, \Omega}^{2}+\int_{\Omega} v \cdot \nabla w w d x+\int_{\Omega} \frac{v_{r}}{r} & w^{2} d x-\nu \int_{\Omega} \Delta w w d x+\nu \int_{\Omega} \frac{w^{2}}{r^{2}} d x \\
& =-\int_{\Omega} \frac{1}{r} q w d x+2 \nu \int_{\Omega} \frac{1}{r^{2}} h_{r} w d x+\int_{\Omega} f_{\varphi} w d x .
\end{aligned}
$$

The second term on the l.h.s. vanishes because it equals

$$
\frac{1}{2} \int_{\Omega} \operatorname{div}\left(v w^{2}\right) d x=\frac{1}{2} \int_{S} v \cdot \bar{n} w^{2} d S=0 .
$$


The fourth term on the l.h.s. assumes the form

$$
-\nu \int_{\Omega} \operatorname{div}(\nabla w w) d x+\nu \int_{\Omega}|\nabla w|^{2} d x=\gamma \int_{S} w^{2} d S-\nu \int_{S} \frac{a_{1}}{r} w^{2} d S+\nu \int_{\Omega}|\nabla w|^{2} d x,
$$

where the boundary condition $(1.5)_{2}$ was used.

We estimate the third term on the l.h.s. of (5.7) by

$$
\varepsilon|w|_{2,-1, \Omega}^{2}+c(1 / \varepsilon)\left|v_{r}\right|_{\infty, \Omega}^{2}|w|_{2, \Omega}^{2}
$$

for any $\varepsilon>0$, and the terms on the r.h.s. by

$$
\varepsilon|w|_{2,-1, \Omega}^{2}+c(1 / \varepsilon)\left(|q|_{2, \Omega}^{2}+|h|_{2,-1, \Omega}^{2}+\left|f_{\varphi}\right|_{2, \Omega}^{2}\right) .
$$

Using the above estimates in (5.7) implies

$$
\begin{aligned}
\frac{1}{2} \frac{d}{d t}|w|_{2, \Omega}^{2}+ & \nu\left|w{ }_{, x}\right|_{2, \Omega}^{2}+\frac{\nu}{2}|w|_{2,-1, \Omega}^{2}+\gamma|w|_{2, S}^{2} \\
& \leq c\left|v_{r}\right|_{\infty, \Omega}^{2}|w|_{2, \Omega}^{2}+\nu \int_{S} \frac{a_{1}}{r} w^{2} d S+c\left(|q|_{2, \Omega}^{2}+|h|_{2,-1, \Omega}^{2}+\left|f_{\varphi}\right|_{2, \Omega}^{2}\right) .
\end{aligned}
$$

Integrating (5.8) with respect to $t$ yields (5.6). This concludes the proof.

Lemma 5.4. Assume that $v \in L_{2}\left(0, T ; L_{\infty}(\Omega)\right), q \in L_{2,-1}\left(\Omega^{T}\right), h \in L_{2,-2}\left(\Omega^{T}\right), f_{\varphi} \in$ $L_{2}\left(\Omega^{T}\right), \int_{S}\left(a_{1} / r\right) w^{2} d S<\infty, w(0) \in H^{1}(\Omega), w(0) \in L_{2,-1}(\Omega), a_{1} \geq 0$. Then solutions of (1.5) satisfy

$$
\begin{aligned}
& \frac{1}{2}\left|w_{t}\right|_{2, \Omega^{t}}^{2}+\frac{\nu}{2}\left|w w_{, x}\right|_{2, \Omega}^{2}+\frac{\gamma}{2}|w|_{2, S}^{2}+\frac{\nu}{2}|w|_{2,-1, \Omega}^{2} \\
& \leq c \exp \left[c \int_{0}^{t}\left|v\left(t^{\prime}\right)\right|_{\infty, \Omega}^{2} d t^{\prime}\right]\left[|q|_{2,-1, \Omega^{t}}^{2}+|h|_{2,-2, \Omega^{t}}^{2}+\left|f_{\varphi}\right|_{2, \Omega^{t}}^{2}\right. \\
& \left.\quad+\frac{\nu}{2}\left|w_{, x}(0)\right|_{2, \Omega}^{2}+\frac{\gamma}{2}|w(0)|_{2, S}^{2}+\frac{\nu}{2}|w(0)|_{2,-1, \Omega}^{2}+\frac{\nu}{2} \int_{S} \frac{a_{1}}{r} w^{2} d S\right], \quad t \leq T .
\end{aligned}
$$

Proof. Multiplying (1.5) $)_{1}$ by $w_{t}$ and integrating over $\Omega$ yields

$$
\begin{aligned}
\left|w_{t}\right|_{2, \Omega}^{2}+\int_{\Omega} v \cdot \nabla w w_{t} d x+\int_{\Omega} \frac{v_{r}}{r} & w w_{t} d x-\nu \int_{\Omega} \Delta w w_{t} d x+\nu \int_{\Omega} \frac{w w_{t}}{r^{2}} d x \\
& =-\int_{\Omega} \frac{1}{r} q w_{t} d x+2 \nu \int_{\Omega} \frac{1}{r^{2}} h_{r} w_{t} d x+\int_{\Omega} f_{\varphi} w_{t} d x .
\end{aligned}
$$

We estimate the second and third terms on the l.h.s. by

$$
\varepsilon\left|w_{t}\right|_{2, \Omega}^{2}+c(1 / \varepsilon)|v|_{\infty, \Omega}^{2}\left(\left|w_{, x}\right|_{2, \Omega}^{2}+|w|_{2,-1, \Omega}^{2}\right)
$$

for any $\varepsilon>0$. The fourth term on the l.h.s. gives

$$
-\nu \int_{\Omega} \operatorname{div}\left(\nabla w w_{t}\right) d x+\frac{\nu}{2} \frac{d}{d t} \int_{\Omega} w_{, x}^{2} d x
$$

where the first term equals

$$
-\nu \int_{S} n \cdot \nabla w w_{t} d S=\gamma \int_{S} w w_{t} d S-\nu \int_{S} \frac{a_{1}}{r} w w_{t} d S=\frac{d}{d t}\left(\frac{\gamma}{2}|w|_{2, S}^{2}-\frac{\nu}{2} \int_{S} \frac{a_{1}}{r} w^{2} d S\right) .
$$


The terms on the r.h.s. of (5.10) are estimated by

$$
\varepsilon\left|w_{t}\right|_{2, \Omega}^{2}+c(1 / \varepsilon)\left(|q|_{2,-1, \Omega}^{2}+|h|_{2,-2, \Omega}^{2}+\left.f_{\varphi}\right|_{2, \Omega} ^{2}\right) .
$$

In view of the above estimates (5.10) takes the form

$$
\begin{aligned}
\frac{1}{2}\left|w_{t}\right|_{2, \Omega}^{2} & +\frac{d}{d t}\left[\frac{\nu}{2}\left|w_{, x}\right|_{2, \Omega}^{2}+\frac{\gamma}{2}|w|_{2, S}^{2}+\frac{\nu}{2}|w|_{2,-1, \Omega}^{2}-\frac{\nu}{2} \int_{S} \frac{a_{1}}{r} w^{2} d S\right] \\
& \leq c|v|_{\infty, \Omega}^{2}\left(\left|w_{, x}\right|_{2, \Omega}^{2}+|w|_{2,-1, \Omega}^{2}\right)+c\left(|q|_{2,-1, \Omega}^{2}+|h|_{2,-2, \Omega}^{2}+\left|f_{\varphi}\right|_{2, \Omega}^{2}\right) .
\end{aligned}
$$

Integrating (5.11) with respect to time yields (5.9). This concludes the proof.

Let us introduce the quantity

$$
A_{1}(t)=|q(t)|_{2,-1, \Omega}^{2}+|h(t)|_{2,-2, \Omega}^{2}+\left|f_{\varphi}(t)\right|_{2, \Omega}^{2} .
$$

Then we have

Lemma 5.5. Assume that $v \in A_{1-\mu}\left(\Omega^{t}\right), \mu \in(1 / 2,1), w \in L_{\infty}\left(0, t ; L_{2,1}(\Omega)\right), w(0) \in$ $H^{1}(\Omega) \cap L_{2,-1}(\Omega), w(0) \in L_{2}(S), t \leq T$ and

$$
\int_{0}^{t} A_{1}\left(t^{\prime}\right) d t^{\prime}<\infty .
$$

Assume either $0<a_{1} \leq c r^{2}$ in a neighbourhood of $r=0$, or $a_{1}<0$ and $\left|a_{1}\right| \leq c r$. Then for solutions of (1.5) we have

$$
\begin{aligned}
\nu\left|w_{, x}(t)\right|_{2, \Omega}^{2}+\nu|w(t)|_{2,-1, \Omega}^{2} & +\gamma|w(t)|_{2, S}^{2} \\
\leq & c e^{c\|v\|_{A_{1-\mu}}^{2}\left(\Omega^{t}\right)}\left[\left(1+\|v\|_{A_{1-\mu}\left(\Omega^{t}\right)}^{4}\right) \sup _{t^{\prime} \leq t}\left|w\left(t^{\prime}\right)\right|_{2,1, \Omega}^{2}\right. \\
& +e^{-t} \int_{0}^{t}\left|w\left(t^{\prime}\right)\right|_{2, \Omega}^{2} e^{t^{\prime}} d t^{\prime}+\int_{0}^{t} A_{1}\left(t^{\prime}\right) d t^{\prime} \\
& \left.+e^{-t}\left(\left|w_{, x}(0)\right|_{2, \Omega}^{2}+|w(0)|_{2,-1, \Omega}^{2}+|w(0)|_{2, S}^{2}\right)\right]
\end{aligned}
$$

Proof. From (5.8) and (5.11) we have

$$
\begin{aligned}
\frac{1}{2} \frac{d}{d t}\left[\nu\left|w_{, x}\right|_{2, \Omega}^{2}+\nu|w|_{2,-1, \Omega}^{2}+\gamma|w|_{2, S}^{2}-\nu \int_{S} \frac{a_{1}}{r} w^{2} d S\right]+\frac{1}{2} \frac{d}{d t}|w|_{2, \Omega}^{2} \\
+\frac{1}{2}\left[\nu\left|w_{, x}\right|_{2, \Omega}^{2}+\nu|w|_{2,-1, \Omega}^{2}+\gamma|w|_{2, S}^{2}-\nu \int_{S} \frac{a_{1}}{r} w^{2} d S\right] \\
+\left(\frac{\nu}{2}\left|w_{, x}\right|_{2, \Omega}^{2}+\frac{\gamma}{2}|w|_{2, S}^{2}\right) \\
\leq c|v|_{\infty, \Omega}^{2}\left(|w|_{2, \Omega}^{2}+|w|_{2, \Omega}^{2}+|w|_{2,-1, \Omega}^{2}\right)+c \int_{S} \frac{a_{1}}{r} w^{2} d S \\
+c\left(|q|_{2,-1, \Omega}^{2}+|h|_{2,-2, \Omega}^{2}+\left|f_{\varphi}\right|_{2, \Omega}^{2}\right) .
\end{aligned}
$$


We reformulate (5.14) as

$$
\begin{aligned}
& \frac{d}{d t}|w|_{2, \Omega}^{2}+\frac{d}{d t}\left[\nu\left|w_{, x}\right|_{2, \Omega}^{2}+\nu|w|_{2,-1, \Omega}^{2}+\gamma|w|_{2, S}^{2}-\nu \int_{S} \frac{a_{1}}{r} w^{2} d S\right] \\
& +\left[\nu\left|w_{, x}\right|_{2, \Omega}^{2}+\nu|w|_{2,-1, \Omega}^{2}+\gamma|w|_{2, S}^{2}-\nu \int_{S} \frac{a_{1}}{r} w^{2} d S\right]+\nu\left|w_{, x}\right|_{2, \Omega}^{2}+\gamma|w|_{2, S}^{2} \\
& \leq c|v|_{\infty, \Omega}^{2}\left[\nu\left|w_{, x}\right|_{2, \Omega}^{2}+\nu|w|_{2,-1, \Omega}^{2}+\gamma|w|_{2, S}^{2}-\nu \int_{S} \frac{a_{1}}{r} w^{2} d S\right] \\
& +c\left(1+|v|_{\infty, \Omega}^{2}\right) \int_{S} \frac{a_{1}}{r} w^{2} d S+c A_{1}(t) .
\end{aligned}
$$

Multiplying (5.15) by $e^{t-c \int_{0}^{t}\left|v\left(t^{\prime}\right)\right|_{\infty, \Omega}^{2} d t^{\prime}}$ yields

$$
\begin{aligned}
& \frac{d}{d t}|w|_{2, \Omega}^{2} e^{t-c \int_{0}^{t}\left|v\left(t^{\prime}\right)\right|_{\infty, \Omega}^{2} d t^{\prime}} \\
& \quad+\frac{d}{d t}\left[\left(\nu\left|w w_{, x}\right|_{2, \Omega}^{2}+\nu|w|_{2,-1, \Omega}^{2}+\gamma|w|_{2, S}^{2}-\nu \int_{S} \frac{a_{1}}{r} w^{2} d S\right) e^{t-c \int_{0}^{t}\left|v\left(t^{\prime}\right)\right|_{\infty, \Omega}^{2} d t^{\prime}}\right] \\
& \quad+\left(\nu\left|w_{, x}\right|_{2, \Omega}^{2}+\gamma|w|_{2, S}^{2}\right) e^{t-c \int_{0}^{t}\left|v\left(t^{\prime}\right)\right|_{\infty, \Omega}^{2} d t^{\prime}} \\
& \leq c\left(1+|v|_{\infty, \Omega}^{2}\right) \int_{S} \frac{a_{1}}{r} w^{2} d S e^{t-c \int_{0}^{t}\left|v\left(t^{\prime}\right)\right|_{\infty, \Omega}^{2} d t^{\prime}} \\
& \quad+c A_{1}(t) e^{t-c \int_{0}^{t}\left|v\left(t^{\prime}\right)\right|_{\infty, \Omega}^{2} d t^{\prime}} .
\end{aligned}
$$

This implies

$$
\begin{aligned}
& \frac{d}{d t}\left(|w|_{2, \Omega}^{2} e^{t-c \int_{0}^{t}\left|v\left(t^{\prime}\right)\right|_{\infty, \Omega}^{2} d t^{\prime}}\right) \\
& \quad+\frac{d}{d t}\left[\left(\nu\left|w_{, x}\right|_{2, \Omega}^{2}+\nu|w|_{2,-1, \Omega}^{2}+\gamma|w|_{2, S}^{2}-\nu \int_{S} \frac{a_{1}}{r} w^{2} d S\right) e^{t-c \int_{0}^{t}\left|v\left(t^{\prime}\right)\right|_{\infty, \Omega}^{2} d t^{\prime}}\right] \\
& \quad+\left(\left.\nu|w|_{, x}\right|_{2, \Omega} ^{2}+\gamma|w|_{2, S}^{2}\right) e^{t-c \int_{0}^{t}\left|v\left(t^{\prime}\right)\right|_{\infty, \Omega}^{2} d t^{\prime}} \\
& \leq c\left(1+|v|_{\infty, \Omega}^{2}\right) \int_{S} \frac{a_{1}}{r} w^{2} d S e^{t-c \int_{0}^{t}\left|v\left(t^{\prime}\right)\right|_{\infty, \Omega}^{2} d t^{\prime}} \\
& \quad+\left(1-c\left|v\left(t^{\prime}\right)\right|_{\infty, \Omega}^{2}\right)|w|_{2, \Omega}^{2} e^{t-c \int_{0}^{t}\left|v\left(t^{\prime}\right)\right|_{\infty, \Omega}^{2} d t^{\prime}} \\
& \quad+c A_{1}(t) e^{t-c \int_{0}^{t}\left|v\left(t^{\prime}\right)\right|_{\infty, \Omega}^{2} d t^{\prime}} .
\end{aligned}
$$

Integrating (5.17) with respect to time yields

$$
\begin{aligned}
|w(t)|_{2, \Omega}^{2} e^{t-c \int_{0}^{t}\left|v\left(t^{\prime}\right)\right|_{\infty, \Omega}^{2} d t^{\prime}} & \\
+ & {\left[\nu\left|w_{, x}(t)\right|_{2, \Omega}^{2}+\nu|w(t)|_{2,-1, \Omega}^{2}+\gamma|w(t)|_{2, S}^{2}-\nu \int_{S} \frac{a_{1}}{r} w^{2}(t) d S\right] } \\
\cdot & e^{t-c \int_{0}^{t}\left|v\left(t^{\prime}\right)\right|_{\infty, \Omega}^{2} d t^{\prime}}
\end{aligned}
$$




$$
\begin{aligned}
& +\int_{0}^{t}\left(\nu\left|w_{, x}\left(t^{\prime}\right)\right|_{2, \Omega}^{2}+\gamma\left|w\left(t^{\prime}\right)\right|_{2, S}^{2}\right) e^{t^{\prime}-c \int_{0}^{t}\left|v\left(t^{\prime \prime}\right)\right|_{\infty, \Omega}^{2} d t^{\prime \prime}} d t^{\prime} \\
\leq & c \int_{0}^{t}\left(1+\left|v\left(t^{\prime}\right)\right|_{\infty, \Omega}^{2}\right) \int_{S} \frac{a_{1}}{r} w^{2}\left(t^{\prime}\right) d S e^{t^{\prime}-c \int_{0}^{t^{\prime}}\left|v\left(t^{\prime \prime}\right)\right|_{\infty, \Omega}^{2}} d t^{\prime \prime} d t^{\prime} \\
& +\int_{0}^{t}\left(1-c\left|v\left(t^{\prime}\right)\right|_{\infty, \Omega}^{2}\right)\left|w\left(t^{\prime}\right)\right|_{2, \Omega}^{2} e^{t^{\prime}-c \int_{0}^{t^{\prime}}\left|v\left(t^{\prime \prime}\right)\right|_{\infty, \Omega}^{2} d t^{\prime \prime}} d t^{\prime} \\
& +c \int_{0}^{t} A_{1}\left(t^{\prime}\right) e^{t^{\prime}-c \int_{0}^{t^{\prime}}\left|v\left(t^{\prime \prime}\right)\right|_{\infty, \Omega}^{2} d t^{\prime \prime}} d t^{\prime} \\
& +|w(0)|_{2, \Omega}^{2}+\nu\left|w_{, x}(0)\right|_{2, \Omega}^{2}+\nu|w(0)|_{2, \Omega}^{2}+\gamma|w(0)|_{2, S}^{2}-\nu \int_{S} \frac{a_{1}}{r} w^{2}(0) d S
\end{aligned}
$$

Assuming that $0<a_{1} \leq c r^{2}$ in a neighbourhood of $r=0$ we obtain

$$
\int_{S} \frac{a_{1}}{r} w^{2} d S \leq c|w|_{2,1 / 2, S}^{2} \leq \varepsilon\left|w_{, x}\right|_{2, \Omega}^{2}+c \varepsilon^{-1}|w|_{2,1, \Omega}^{2}
$$

for any $\varepsilon>0$. Using (5.19) for $0<a_{1}<c r^{2}$ we have the inequality

$$
|v|_{\infty, \Omega}^{2} \int_{S} \frac{a_{1}}{r} w^{2} d S \leq \varepsilon\left|w_{, x}\right|_{2, \Omega}^{2}+c \varepsilon^{-1}|v|_{\infty, \Omega}^{4}|w|_{2,1, \Omega}^{2}
$$

In view of (5.20) we obtain from (5.18) the inequality

$$
\begin{aligned}
|w(t)|_{2, \Omega}^{2} e^{t-c \int_{0}^{t}\left|v\left(t^{\prime}\right)\right|_{\infty, \Omega}^{2} d t^{\prime}} & +\left[\nu\left|w_{, x}(t)\right|_{2, \Omega}^{2}+\nu|w(t)|_{2,-1, \Omega}^{2}+\gamma|w(t)|_{2, S}^{2}-\nu \int_{S} \frac{a_{1}}{r} w^{2}(t) d S\right] \\
& \cdot e^{t-c \int_{0}^{t}\left|v\left(t^{\prime}\right)\right|_{\infty, \Omega}^{2} d t^{\prime}} \\
& +\int_{0}^{t}\left[(\nu-\varepsilon)\left|w_{, x}\left(t^{\prime}\right)\right|_{2, \Omega}^{2}+\gamma\left|w\left(t^{\prime}\right)\right|_{2, S}^{2}\right] e^{t^{\prime}-c \int_{0}^{t^{\prime}}\left|v\left(t^{\prime \prime}\right)\right|_{\infty, \Omega}^{2} d t^{\prime \prime}} d t^{\prime} \\
\leq & c \varepsilon^{-1} \int_{0}^{t}\left(1+\left|v\left(t^{\prime}\right)\right|_{\infty, \Omega}^{4}\right)\left|w\left(t^{\prime}\right)\right|_{2,1, \Omega}^{2} e^{t^{\prime}-c \int_{0}^{t^{\prime}}\left|v\left(t^{\prime \prime}\right)\right|_{\infty, \Omega}^{2} d t^{\prime \prime}} d t^{\prime} \\
& +c \int_{0}^{t}\left(1-c\left|v\left(t^{\prime}\right)\right|_{\infty, \Omega}^{2}\right)\left|w\left(t^{\prime}\right)\right|_{2, \Omega}^{2} e^{t^{\prime}-c \int_{0}^{t^{\prime}}\left|v\left(t^{\prime \prime}\right)\right|_{\infty, \Omega}^{2} d t^{\prime \prime}} d t^{\prime} \\
& +c \int_{0}^{t} A_{1}\left(t^{\prime}\right) e^{t^{\prime}-c \int_{0}^{t^{\prime}}\left|v\left(t^{\prime \prime}\right)\right|_{\infty, \Omega}^{2} d t^{\prime \prime}} d t^{\prime}+|w(0)|_{2, \Omega}^{2}+\nu\left|w_{, x}(0)\right|_{2, \Omega}^{2} \\
& +\nu|w(0)|_{2,-1, \Omega}^{2}+\gamma|w(0)|_{2, S}^{2}-\nu \int_{S} \frac{a_{1}}{r} w^{2}(0) d S,
\end{aligned}
$$

where the last term can be omitted for $a_{1} \geq 0$. For $a_{1}<0$ we use the fact that $\left|a_{1}\right| \leq c r$, and the last term on the r.h.s. of (5.21) is estimated by $c|w(0)|_{2, S}^{2}$. 
In the case $a_{1} \leq 0$ the first term on the r.h.s. of (5.21) can be cancelled. Assuming that $\varepsilon$ is sufficiently small we can omit the last term on the l.h.s. of (5.21). Multiplying the result by $e^{-t+c \int_{0}^{t}\left|v\left(t^{\prime}\right)\right|_{\infty, \Omega}^{2} d t^{\prime}}$ and exploiting again (5.19) we have

$$
\begin{aligned}
& \nu|w, x(t)|_{2, \Omega}^{2}+\nu|w(t)|_{2,-1, \Omega}^{2}+\gamma|w(t)|_{2, S}^{2} \\
& \leq c e^{c \int_{0}^{t}\left|v\left(t^{\prime}\right)\right|_{\infty, \Omega}^{2} d t^{\prime}} \sup _{t^{\prime} \leq t}\left|w\left(t^{\prime}\right)\right|_{2,1, \Omega}^{2}\left[1+\int_{0}^{t}\left|v\left(t^{\prime}\right)\right|_{\infty, \Omega}^{4} d t^{\prime}\right] \\
& \quad+c e^{-t+c \int_{0}^{t}\left|v\left(t^{\prime}\right)\right|_{\infty, \Omega}^{2} d t^{\prime}} \int_{0}^{t}\left|w\left(t^{\prime}\right)\right|_{2, \Omega}^{2} e^{t^{\prime}} d t^{\prime}+c e^{c \int_{0}^{t}\left|v\left(t^{\prime}\right)\right|_{\infty, \Omega}^{2} d t^{\prime}} \int_{0}^{t} A_{1}\left(t^{\prime}\right) d t^{\prime} \\
& \quad+c e^{-t+c \int_{0}^{t}\left|v\left(t^{\prime}\right)\right|_{\infty, \Omega}^{2} d t^{\prime}}\left[\left|w_{, x}(0)\right|_{2, \Omega}^{2}+|w(0)|_{2,-1, \Omega}^{2}+|w(0)|_{2, S}^{2}\right] .
\end{aligned}
$$

Using the estimate

$$
\left(\int_{0}^{t}\left|v\left(t^{\prime}\right)\right|_{\infty, \Omega}^{4} d t^{\prime}\right)^{1 / 4} \leq c\|v\|_{A_{1-\mu}\left(\Omega^{T}\right)}, \quad \mu \in(1 / 2,1),
$$

we obtain (5.13). This concludes the proof.

Let us introduce the quantity

$$
A_{2}(t)=|q(t)|_{2, \Omega}+|h(t)|_{2,-1, \Omega}+\left|f_{\varphi}(t)\right|_{2, \Omega} .
$$

Lemma 5.6. Assume that

$$
\int_{0}^{t} A_{2}\left(t^{\prime}\right) d t^{\prime}<\infty, \quad|w(0)|_{2,1, \Omega}<\infty .
$$

Then for solutions of (1.5) we obtain

$$
|w(t)|_{2,1, \Omega} \leq|w(0)|_{2,1, \Omega}+\int_{0}^{t} A_{1}\left(t^{\prime}\right) d t^{\prime}, \quad t \in \mathbb{R}_{+} .
$$

Proof. Multiplying (1.5) $)_{1}$ by $w r^{2 \mu}$ and integrating over $\Omega$ we obtain

$$
\begin{aligned}
\frac{1}{2} \frac{d}{d t}|w|_{2, \mu, \Omega}^{2}+\int_{\Omega} v \cdot \nabla w w r^{2 \mu} d x+\int_{\Omega} \frac{v_{r}}{r} w^{2} r^{2 \mu} d x \\
\quad-\nu \int_{\Omega} \Delta w w r^{2 \mu} d x+\nu|w|_{2, \mu-1, \Omega}^{2} \\
=-\int_{\Omega} \frac{1}{r} q w r^{2 \mu} d x+2 \nu \int_{\Omega} \frac{1}{r^{2}} h_{r} w r^{2 \mu} d x+\int_{\Omega} f_{\varphi} w r^{2 \mu} d x .
\end{aligned}
$$

The sum of the second and third terms on the l.h.s. equals

$$
(1-\mu) \int_{\Omega} \frac{v_{r}}{r} w^{2} r^{2 \mu} d x .
$$

The fourth term on the l.h.s. of (5.25) takes the form

$$
-\nu \int_{\Omega} \operatorname{div}\left(\nabla w w r^{2 \mu}\right) d x+\nu \int_{\Omega} w_{, x}^{2} r^{2 \mu} d x+2 \mu \nu \int_{\Omega} \nabla w w r^{2 \mu-1} \nabla r d x,
$$


where by using the boundary condition $(1.5)_{2}$ the first term is expressed in the form

$$
-\nu \int_{S} \bar{n} \cdot \nabla w w r^{2 \mu} d S=\gamma|w|_{2, \mu, S}^{2}-\nu \int_{S} \frac{a_{1}}{r} w^{2} r^{2 \mu} d S
$$

and the last term is handled as follows:

$$
\begin{aligned}
\mu \nu \int_{\Omega} \nabla w^{2} r^{2 \mu-1} \nabla r d x= & \mu \nu \int_{\Omega} \nabla\left(w^{2} r^{2 \mu-1} \nabla r\right) d x \\
& -\mu \nu(2 \mu-1) \int_{\Omega} w^{2} r^{2 \mu-2} \nabla r \cdot \nabla r d x-\mu \nu \int_{\Omega} w^{2} r^{2 \mu-1} \Delta r d x \\
= & \mu \nu \int_{S} \frac{a_{1}}{r} w^{2} r^{2 \mu} d S-2 \mu^{2} \nu|w|_{2, \mu-1, \Omega}^{2} .
\end{aligned}
$$

Finally, the fourth term on the l.h.s. of (5.25) equals

$$
-\nu \int_{\Omega} \Delta w w r^{2 \mu} d x=-\nu \int_{\Omega} \operatorname{div}\left(\nabla w w r^{2 \mu}\right) d x+\nu \int_{\Omega} \nabla w \nabla\left(w r^{2 \mu}\right) d x \equiv I_{1}+I_{2},
$$

where

$$
\begin{aligned}
& I_{1}=-\nu \int_{S} \bar{n} \cdot \nabla w w r^{2 \mu} d S=\gamma \int_{S} w^{2} r^{2 \mu} d S-\nu \int_{S} \frac{a_{1}}{r} w^{2} r^{2 \mu} d S \\
& I_{2}=\nu \int_{\Omega}|\nabla w|^{2} r^{2 \mu} d x+2 \mu \nu \int_{\Omega} \nabla w \cdot \nabla r w r^{2 \mu-1} d x \equiv I_{3}+I_{4} .
\end{aligned}
$$

We have to examine

$$
\begin{aligned}
I_{4} & =2 \mu \nu \int_{\Omega} \partial_{r} w w r^{2 \mu} d r d z d \varphi=\mu \nu \int_{\Omega} \partial_{r} w^{2} r^{2 \mu} d r d z d \varphi \\
& =\mu \nu \int_{\Omega} \partial_{r}\left(w^{2} r^{2 \mu}\right) d r d z d \varphi-2 \mu^{2} \nu \int_{\Omega} w^{2} r^{2 \mu-1} d r d z d \varphi
\end{aligned}
$$

where the first integral on the r.h.s. equals

$$
\begin{aligned}
& \mu \nu \int_{\Omega}\left[\partial_{r}\left(w^{2} r^{2 \mu-1}\right)+\frac{w^{2} r^{2 \mu-1}}{r}\right] r d r d z d \varphi \\
& =\mu \nu \int_{\Omega} \operatorname{div}\left(w^{2} r^{2 \mu-1} \bar{e}_{r}\right) d x=\mu \nu \int_{S} \bar{n} \cdot \bar{e}_{r} w^{2} r^{2 \mu-1} d S=\mu \nu \int_{S} \frac{a_{1}}{r} w^{2} r^{2 \mu} d S
\end{aligned}
$$

Hence the fourth term on the 1.h.s. of (5.25) takes the form

$$
\nu(\mu-1) \int_{S} \frac{a_{1}}{r} w^{2} r^{2 \mu} d S-2 \mu^{2} \nu|w|_{2, \mu-1, \Omega}^{2}+\gamma|w|_{2, \mu, S}^{2}+\nu\left|w_{, x}\right|_{2, \mu, \Omega}^{2} .
$$

In view of the above considerations (5.25) assumes the form

$$
\begin{aligned}
& \frac{1}{2} \frac{d}{d t}|w|_{2, \mu, \Omega}^{2}+\nu\left|w_{, x}\right|_{2, \mu, \Omega}^{2}+\nu\left(1-2 \mu^{2}\right)|w|_{2, \mu-1, \Omega}^{2}+\gamma|w|_{2, \mu, S}^{2} \\
&+(1-\mu) \int_{\Omega} \frac{v_{r}}{r} w^{2} r^{2 \mu} d x+\nu(\mu-1) \int_{S} \frac{a_{1}}{r} w^{2} r^{2 \mu} d S \\
& \leq|w|_{2, \mu, \Omega}\left(|q|_{2, \mu-1, \Omega}+|h|_{2, \mu-2, \Omega}+\left|f_{\varphi}\right|_{2, \mu, \Omega}\right) .
\end{aligned}
$$


Using the Hardy inequality

$$
\mu^{2}|w|_{2, \mu-1, \Omega}^{2} \leq\left|w_{, x}\right|_{2, \mu, \Omega}^{2},
$$

we obtain from (5.26) the inequality

(5.28) $\quad \frac{1}{2} \frac{d}{d t}|w|_{2, \mu, \Omega}^{2}+\nu\left(1-\mu^{2}\right)|w|_{2, \mu-1, \Omega}^{2}+\gamma|w|_{2, \mu, S}^{2}$

$$
\begin{aligned}
& \quad+(1-\mu) \int_{\Omega} \frac{v_{r}}{r} w^{2} r^{2 \mu} d x+\nu(\mu-1) \int_{S} \frac{a_{1}}{r} w^{2} r^{2 \mu} d S \\
& \leq|w|_{2, \mu, \Omega}\left(|q|_{2, \mu-1, \Omega}+|h|_{2, \mu-2, \Omega}+\left|f_{\varphi}\right|_{2, \mu, \Omega}\right) .
\end{aligned}
$$

Inserting $\mu=1$ in (5.28) yields

$$
\frac{1}{2} \frac{d}{d t}|w|_{2,1, \Omega}^{2}+\gamma|w|_{2,1, S}^{2} \leq A_{2}(t)|w|_{2,1, \Omega}
$$

Hence we have

$$
\frac{d}{d t}|w|_{2,1, \Omega} \leq A_{2}(t)
$$

Integrating (5.30) with respect to $t$ gives (5.24). This concludes the proof. 


\section{Local existence: boundedness of the approximating sequence}

We construct successive approximations and show that elements of the approximating sequence are uniformly bounded.

6.1. Formulation of the method of successive approximations. To prove the existence of local solutions to problem (1.1) we use the following method of successive approximations. Let $v_{m}$ be given. Then $h_{m}$ and $q_{m}$ are solutions to the problem

$$
\begin{array}{ll}
h_{m, t}-\nu \operatorname{div} \mathbb{D}\left(h_{m}\right)+\nabla q_{m}+v_{m} \cdot \nabla h_{m}+h_{m} \cdot \nabla v_{m}=g & \text { in } \Omega^{T}, \\
\operatorname{div} h_{m}=0 & \text { in } \Omega^{T}, \\
h_{m} \cdot \bar{n}=0 & \text { on } S^{T}, \\
\bar{n} \cdot \mathbb{D}\left(h_{m}\right) \cdot \bar{\tau}_{\alpha}+\frac{\gamma}{\nu} h_{m} \cdot \bar{\tau}_{\alpha}=0, \quad \alpha=1,2, & \text { on } S^{T}, \\
\left.h_{m}\right|_{t=0}=h(0) & \text { in } \Omega .
\end{array}
$$

Let $v_{m}, q_{m}, h_{m}$ be given. Then $w_{m}$ satisfies

$$
\begin{array}{ll}
w_{m, t}+v_{m} \cdot \nabla w_{m}+\frac{v_{m r}}{r} w_{m}-\nu \Delta w_{m}+\nu \frac{w_{m}}{r^{2}} \\
=\frac{q_{m}}{r}+\frac{2 \nu}{r^{2}} h_{m r}+f_{\varphi} & \text { in } \Omega^{T}, \\
\bar{n} \cdot \nabla w_{m}=-\left(\frac{\gamma}{\nu}-\frac{a_{1}}{r}\right) w_{m} & \text { on } S^{T}, \\
\left.w_{m}\right|_{t=0}=w(0) & \text { in } \Omega .
\end{array}
$$

Let $v_{m}, q_{m}, h_{m}, w_{m}$ be given. Then $\alpha_{m+1}^{\prime}$ is a solution to the problem

$$
\begin{array}{ll}
\alpha_{1 m+1, t}+v_{m} \cdot \nabla \alpha_{1 m+1}-\alpha_{1 m+1} v_{m r, r}-\frac{\alpha_{2 m+1}}{r} h_{m r}-\alpha_{3 m+1} v_{m r, z} & \\
\quad+\frac{2 \nu}{r^{2}}\left(h_{m r, z}-h_{m z, r}\right)+\frac{\nu \alpha_{1 m+1}}{r^{2}}-\nu \Delta \alpha_{1 m+1}=F_{1} & \text { in } \Omega^{T}, \\
\alpha_{3 m+1, t}+v_{m} \cdot \nabla \alpha_{3 m+1}-\left(\alpha_{1 m+1} v_{m z, r}+\alpha_{3 m+1} v_{m z, z}\right) & \\
\quad-\frac{\alpha_{2 m+1}}{r} h_{m z}-\nu \Delta \alpha_{3 m+1}=F_{3} & \text { in } \Omega^{T}, \\
\bar{\tau}_{2} \cdot \alpha_{m+1}^{\prime}=-\frac{2 a_{1}}{r} w_{m} & \text { on } S^{T}, \\
\left(\bar{n} \cdot \alpha_{m+1}^{\prime}\right)_{, n}=\beta_{1} h_{m r}+\beta_{2} h_{m z}+\beta_{3} w_{m, r}+\beta_{4} w_{m, z}+\beta_{5} w_{m} & \text { on } S^{T}, \\
\left.\alpha_{1 m+1}\right|_{t=0}=\alpha_{1}(0),\left.\quad \alpha_{3 m+1}\right|_{t=0}=\alpha_{3}(0) & \text { in } \Omega,
\end{array}
$$


and $\chi_{m+1}=\alpha_{2 m+1}$ is a solution to the problem

$$
\begin{aligned}
& \chi_{m+1, t}+v_{m} \cdot \nabla \chi_{m+1}-\frac{h_{m \varphi}}{r} \chi_{m+1}-\frac{v_{m r}}{r} \chi_{m+1}-\nu \Delta \chi_{m+1}+\nu \frac{\chi_{m+1}}{r^{2}} \\
& =-\frac{1}{r}\left(w_{m, z} h_{m r}-w_{m, r} h_{m z}+\frac{w_{m}}{r} h_{m z}\right)+\frac{2 w_{m} v_{m \varphi, z}}{r} \\
& +\frac{2 \nu}{r^{2}}\left(\frac{1}{r} h_{m z, \varphi}-h_{m \varphi, z}\right)+F_{2} \quad \text { in } \Omega^{T}, \\
& \chi_{m+1}=2(k-\gamma /(2 \nu)) v_{m} \cdot \bar{\tau}_{2} \quad \text { on } S^{T}, \\
& \left.\chi_{m+1}\right|_{t=0}=\alpha_{2}(0) \quad \text { in } \Omega .
\end{aligned}
$$

For a given $v_{m}$ we calculate $p_{m}$ from the elliptic problem

$$
\begin{array}{ll}
\Delta p_{m}=-\nabla v_{m} \cdot \nabla v_{m}+\operatorname{div} f & \text { in } \Omega, \\
\frac{\partial p_{m}}{\partial n}=f \cdot \bar{n}+\nu \bar{n} \cdot \Delta v_{m}-\bar{n} \cdot v_{m} \cdot \nabla v_{m} & \text { on } S .
\end{array}
$$

Finally, in the next step we calculate $v_{m+1}$ from the elliptic problem for a given $\alpha_{m+1}$,

$$
\begin{array}{ll}
\operatorname{rot} v_{m+1}=\alpha_{m+1} & \text { in } \Omega, \\
\operatorname{div} v_{m+1}=0 & \text { in } \Omega, \\
v_{m+1} \cdot \bar{n}=0 & \text { on } S .
\end{array}
$$

6.2. Estimate of the first step. To start the above method of successive approximations we assume that $v_{0}=0$. Then $h_{0}$ and $q_{0}$ are solutions to the problem

$$
\begin{aligned}
& h_{0, t}-\nu \operatorname{div} \mathbb{D}\left(h_{0}\right)+\nabla q_{0}=g \quad \text { in } \Omega^{T}, \\
& \operatorname{div} h_{0}=0 \quad \text { in } \Omega^{T}, \\
& h_{0} \cdot \bar{n}=0 \quad \text { on } S^{T}, \\
& \bar{n} \cdot \mathbb{D}\left(h_{0}\right) \cdot \bar{\tau}_{\alpha}+\frac{\gamma}{\nu} h_{0} \cdot \bar{\tau}_{\alpha}=0, \quad \alpha=1,2, \quad \text { on } S^{T}, \\
& \left.h_{0}\right|_{t=0}=h(0) \quad \text { in } \Omega .
\end{aligned}
$$

Having $h_{0}$ and $q_{0}$ we calculate $w_{0}$ from the problem

$$
\begin{array}{ll}
w_{0, t}-\nu \Delta w_{0}+\nu \frac{w_{0}}{r^{2}}=\frac{q_{0}}{r}+\frac{2 \nu}{r^{2}} h_{0}+f_{\varphi} & \text { in } \Omega^{T}, \\
\bar{n} \cdot \nabla w_{0}=-\left(\frac{\gamma}{\nu}-\frac{a_{1}}{r}\right) w_{0} & \text { on } S^{T}, \\
\left.w_{0}\right|_{t=0}=w(0) & \text { in } \Omega .
\end{array}
$$

Next we calculate $\alpha_{1}$ from the following system of problems:

$$
\begin{array}{ll}
\alpha_{11, t}-\frac{\alpha_{21}}{r} h_{0 r}+\frac{2 \nu}{r^{2}}\left(h_{0 r, z}-h_{0 z, r}\right)+\frac{\nu \alpha_{11}}{r^{2}}-\nu \Delta \alpha_{11}=F_{1} & \text { in } \Omega^{T}, \\
\alpha_{31, t}-\frac{\alpha_{21}}{r} h_{0 z}-\nu \Delta \alpha_{31}=F_{3} & \text { in } \Omega^{T}, \\
\bar{\tau}_{2} \cdot \alpha_{1}^{\prime}=-\frac{2 a_{1}}{r} w_{0} & \text { on } S^{T}, \\
\left(\bar{n} \cdot \alpha_{1}^{\prime}\right)_{, n}=\beta_{1} h_{0 r}+\beta_{2} h_{0 z}+\beta_{3} w_{0, r}+\beta_{4} w_{0, z}+\beta_{5} w_{0} & \text { on } S^{T}, \\
\left.\alpha_{11}\right|_{t=0}=\alpha_{1}(0),\left.\quad \alpha_{31}\right|_{t=0}=\alpha_{3}(0) & \text { in } \Omega,
\end{array}
$$


and

$$
\begin{array}{ll}
\chi_{1, t}-\frac{h_{0 \varphi}}{r} \chi_{1}-\nu \Delta \chi_{1}+\nu \frac{\chi_{1}}{r^{2}}=-\frac{1}{r}\left(w_{0, z} h_{0 r}-w_{0, r} h_{0 z}+\frac{w_{0}}{r} h_{0 z}\right) & \text { in } \Omega^{T}, \\
\quad+\frac{2 \nu}{r^{2}}\left(\frac{1}{r} h_{0 z, \varphi}-h_{0 \varphi, z}\right)+F_{2} & \text { on } S^{T}, \\
\chi_{1}=0 & \text { in } \Omega, \\
\left.\chi_{1}\right|_{t=0}=\alpha_{2}(0) \equiv \chi(0) &
\end{array}
$$

where in the matrix $\alpha_{i j}$ the index $i$ corresponds to the coordinate of the vector $\alpha$ and $j$ corresponds to the step considered.

For the first step we calculate $v_{1}$ from the problem

$$
\begin{array}{ll}
\operatorname{rot} v_{1}=\alpha_{1} & \text { in } \Omega, \\
\operatorname{div} v_{1}=0 & \text { in } \Omega, \\
v_{1} \cdot \bar{n}=0 & \text { on } S .
\end{array}
$$

The aim of this section is to show that the constructed sequence is bounded. For this purpose we introduce the quantities

$$
K_{m}(t)=\left\|v_{m}\right\|_{A_{1-\mu}\left(\Omega^{t}\right)}, \quad m \in \mathbb{N}, \mu \in(1 / 2,1) .
$$

We show that they are bounded by the same constant. From the above considerations we have $K_{0}=\left\|v_{0}\right\|_{A_{1-\mu}\left(\Omega^{t}\right)}=0$.

First we find a bound for $K_{1}$.

Lemma 6.1. Assume that $g \in L_{2,-1}\left(\Omega^{T}\right), f_{\varphi} \in L_{2}\left(\Omega^{T}\right), h(0) \in H_{-1}^{1}(\Omega), w(0) \in H_{0}^{1}(\Omega)$, $\alpha^{\prime}(0) \in W_{2,1-\mu}^{1}(\Omega), \alpha_{1}(0) \in L_{2,-\mu}(\Omega), F^{\prime} \in L_{2}\left(\Omega^{T}\right), F_{1} \in L_{2,-\mu}\left(\Omega^{T}\right), \mu \in(1 / 2,1)$, $F_{2} \in L_{2,-1}\left(\Omega^{T}\right), \chi(0) \in L_{2,-1}(\Omega)$. Let

$$
\begin{aligned}
\widetilde{X}_{1}(t) & =|g|_{2,-1, \Omega^{t}}+\left|f_{\varphi}\right|_{2, \Omega^{t}}+\|h(0)\|_{1,-1, \Omega}+\|w(0)\|_{1,0, \Omega} \leq 1, \\
\widetilde{X}_{2}(t) & =\left|F^{\prime}\right|_{2, \Omega^{t}}+\left|F_{1}\right|_{2,-\mu, \Omega^{t}}+\left\|\alpha^{\prime}(0)\right\|_{1,2,1-\mu, \Omega}+\left|\alpha_{1}(0)\right|_{2,-\mu, \Omega}, \\
\widetilde{Y}_{1}(t) & =\left|F_{2}\right|_{2,-1, \Omega^{t}}+|\chi(0)|_{2,-1, \Omega} .
\end{aligned}
$$

Assume that there exist constants $A$ and $c$ such that

$$
c\left[\left(e^{c t} \widetilde{X}_{1}(t)+\widetilde{Y}_{1}(t)\right) t^{1 / 2} \widetilde{X}_{1}(t)+\tilde{X}_{2}(t)+\widetilde{Y}_{1}(t)\right]<A, \quad t \leq T .
$$

Then

$$
\left\|v_{1}\right\|_{A_{1-\mu}\left(\Omega^{t}\right)} \leq A, \quad t \leq T
$$

and $T$ is defined by $\left(6.12^{\prime}\right)$.

Proof. For solutions of problem (6.11) we have

$$
K_{1}(t)=\left\|v_{1}\right\|_{A_{1-\mu}\left(\Omega^{t}\right)} \leq c\left\|\alpha_{1}\right\|_{2,2,1-\mu, \Omega^{t}} .
$$

Now we estimate the r.h.s. of the above inequality. Applying Lemma 4.2 to problem (6.7) yields

$$
\left\|h_{0}\right\|_{2,-1, \Omega^{t}}+\left\|q_{0}\right\|_{1,-1, \Omega^{t}} \leq c\left(|g|_{2,-1, \Omega^{t}}+\|h(0)\|_{1,-1, \Omega}\right) .
$$


Applying Lemma 5.1 to problem (6.8) gives

(6.16) $\quad\left\|w_{0}\right\|_{2,1-\mu, \Omega^{t}}$

$$
\leq c\left(|g|_{2,-1, \Omega^{t}}+\|h(0)\|_{1,-1, \Omega}+\|w(0)\|_{1,1-\mu, \Omega}+\left|f_{\varphi}\right|_{2,1-\mu, \Omega^{t}}+\left|w_{0}\right|_{2,-(1+\mu), \Omega^{t}}\right) .
$$

To estimate the last term on the r.h.s. of (6.16) we use Lemma 5.2. Hence we have

$$
\begin{aligned}
\left|w_{0}(t)\right|_{2,-\mu, \Omega}^{2} & +\left|\nabla w_{0}\right|_{2,-\mu, \Omega^{t}}^{2}+\left|w_{0}\right|_{2,-(1+\mu), \Omega^{t}}^{2}+\left|w_{0}\right|_{2,-\mu, S^{t}}^{2} \\
\leq & c e^{c t}\left(\left|q_{0}\right|_{2,-\mu, \Omega^{t}}^{2}+\left|h_{0}\right|_{2,-(1+\mu), \Omega^{t}}^{2}+\left|f_{\varphi}\right|_{2,1-\mu, \Omega^{t}}^{2}\right)+|w(0)|_{2,-\mu, \Omega}^{2} \\
\leq & c e^{c t}\left(|g|_{2,-1, \Omega^{t}}^{2}+\|h(0)\|_{1,-1, \Omega}^{2}+\left|f_{\varphi}\right|_{2,1-\mu, \Omega^{t}}^{2}\right)+|w(0)|_{2,-\mu, \Omega}^{2},
\end{aligned}
$$

where the last inequality follows from (6.15).

Making use of (6.17) in (6.16) yields

$$
\left\|w_{0}\right\|_{2,1-\mu, \Omega^{t}} \leq c e^{c t}\left(|g|_{2,-1, \Omega^{t}}+\|h(0)\|_{1,-1, \Omega}+\left|f_{\varphi}\right|_{2,1-\mu, \Omega^{t}}\right)+c\|w(0)\|_{1,1-\mu, \Omega} .
$$

Next we obtain an estimate for $\alpha_{1}^{\prime}=\left(\alpha_{11}, \alpha_{31}\right)$. In view of Lemma 4.3 we have

$$
\begin{aligned}
& \left\|\alpha_{1}^{\prime}\right\|_{2,2,1-\mu, \Omega^{t}} \leq c\left|\alpha_{21} / r\right|_{10 / 3, \Omega^{t}}\left\|h_{0}\right\|_{2,-\mu, \Omega^{t}} \\
& \quad+c\left(\left\|h_{0}\right\|_{2,-\mu, \Omega^{t}}+\left\|w_{0}\right\|_{2,1-\mu, \Omega^{t}}\right)+c\left|\alpha_{11}\right|_{2,-(1+\mu), \Omega^{t}}+c\left|F^{\prime}\right|_{2,1-\mu, \Omega^{t}} .
\end{aligned}
$$

Employing estimates (6.15) and (6.18) in (6.19) yields

$$
\begin{aligned}
\left\|\alpha_{1}^{\prime}\right\|_{2,2,1-\mu, \Omega^{t}} \leq & c\left|\alpha_{21} / r\right|_{10 / 3, \Omega^{t}}\left(|g|_{2,-1, \Omega^{t}}+\|h(0)\|_{1,-1, \Omega}\right) \\
& +c e^{c t}\left(|g|_{2,-1, \Omega^{t}}+\|h(0)\|_{1,-1, \Omega}+\left|f_{\varphi}\right|_{2,1-\mu, \Omega^{t}}\right) \\
& +c\|w(0)\|_{1,1-\mu, \Omega}+c\left|F^{\prime}\right|_{2,1-\mu, \Omega^{t}}+c\left|\alpha_{11}\right|_{2,-(1+\mu), \Omega^{t}} .
\end{aligned}
$$

In view of Lemma 4.5 and $(6.15),(6.18)$ we have

$$
\begin{aligned}
\left|\alpha_{11}\right|_{2,-(1+\mu), \Omega^{t}} & \leq c\left(\left|\alpha_{21} / r\right|_{10 / 3, \Omega^{t}}+1\right)\left(|g|_{2,-1, \Omega^{t}}+\|h(0)\|_{1,-1, \Omega}\right) \\
& +c e^{c t}\left(|g|_{2,-1, \Omega^{t}}+\|h(0)\|_{1,-1, \Omega}+\left|f_{\varphi}\right|_{2,1-\mu, \Omega^{t}}\right) \\
& +c\left(\left|F^{\prime}\right|_{2, \Omega^{t}}+\left|F_{1}\right|_{2,-\mu, \Omega^{t}}\right)+c\left(\left\|\alpha^{\prime}(0)\right\|_{1,2,1-\mu, \Omega}+\left|\alpha_{1}(0)\right|_{2,-\mu, \Omega}\right) \\
& +c\|w(0)\|_{1,1-\mu, \Omega} \leq c\left(\left|\alpha_{21} / r\right|_{10 / 3, \Omega^{t}}+e^{c t}\right)\left[X_{1}^{\prime}(t)+X_{2}^{\prime}\right] \\
& +c\left(X_{3}^{\prime}+X_{4}^{\prime}(t)\right),
\end{aligned}
$$

where we introduced the quantities

$$
\begin{aligned}
X_{1}^{\prime}(t) & =|g|_{2,-1, \Omega^{t}}+\left|f_{\varphi}\right|_{2, \Omega^{t}}, \\
X_{2}^{\prime} & =\|h(0)\|_{1,-1, \Omega}+\|w(0)\|_{1,0, \Omega}, \\
X_{3}^{\prime} & =\left\|\alpha^{\prime}(0)\right\|_{1,2,1-\mu, \Omega}+\left|\alpha_{1}(0)\right|_{2,-\mu, \Omega}, \\
X_{4}^{\prime}(t) & =\left|F^{\prime}\right|_{2, \Omega^{t}}+\left|F_{1}\right|_{2,-\mu, \Omega^{t}}, \\
Y_{1}^{\prime}(t) & =\left|F_{2}\right|_{2,-1, \Omega^{t}}, \\
Y_{2}^{\prime} & =|\chi(0)|_{2,-1, \Omega}+\|\chi(0)\|_{1,1-\mu, \Omega} .
\end{aligned}
$$

Employing (6.21) in (6.20) yields

$$
\left\|\alpha_{1}^{\prime}\right\|_{2,2,1-\mu, \Omega^{t}} \leq c\left(\left|\alpha_{21} / r\right|_{10 / 3, \Omega^{t}}+e^{c t}\right)\left(X_{1}^{\prime}(t)+X_{2}^{\prime}\right)+c\left(X_{3}^{\prime}+X_{4}^{\prime}(t)\right) .
$$


To obtain an estimate for $\alpha_{21}$ we formulate the problem for $\chi_{1}$ :

$$
\begin{array}{ll}
\chi_{1, t}-\nu\left[\left(r\left(\frac{\chi_{1}}{r}\right)_{, r}\right)_{, r}+\frac{1}{r^{2}} \chi_{1, \varphi \varphi}+\chi_{1, z z}+2\left(\frac{\chi_{1}}{r}\right)_{, r}\right] & \\
\quad-\frac{2 \nu}{r^{2}}\left(\frac{1}{r} h_{0 z, \varphi}-h_{0 \varphi, z}\right)-\frac{1}{r}\left(w_{0, z} h_{0 r}-w_{0, r} h_{0 z}+\frac{w_{0}}{r} h_{0 z}\right)=F_{1} & \text { in } \Omega^{T}, \\
\chi_{1}=0 & \text { on } S^{T}, \\
\left.\chi_{1}\right|_{t=0}=\chi(0) & \text { in } \Omega .
\end{array}
$$

For solutions of (6.24) we have the estimate

$$
\begin{aligned}
\sup _{t}\left|\chi_{1} / r\right|_{2, \Omega}^{2}+ & \nu \int_{0}^{t}\left\|\chi_{1} / r\right\|_{1, \Omega}^{2} d t^{\prime} \leq c \sup _{t}\left\|h_{0 \varphi}\right\|_{1,-1, \Omega} \int_{0}^{t}\left\|\chi_{1} / r\right\|_{1, \Omega}^{2} d t^{\prime} \\
& +c\left(1+\sup _{t}\left\|w_{0}\right\|_{1,0, \Omega}^{2}\right)\left\|h_{0}\right\|_{2,-1, \Omega^{t}}^{2}+c\left|F_{2}\right|_{2,-1, \Omega^{t}}^{2}+|\chi(0)|_{2,-1, \Omega}^{2} .
\end{aligned}
$$

Using (6.15) in (6.25) and assuming that the r.h.s. of (6.15) is sufficiently small we obtain from (6.25) the inequality

$$
\begin{gathered}
\sup _{t}\left|\chi_{1}\right|_{2,-1, \Omega}^{2}+\nu \int_{0}^{t}\left\|\chi_{1} / r\right\|_{1, \Omega}^{2} d t^{\prime} \leq c\left(1+\sup _{t}\left\|w_{0}\right\|_{1,0, \Omega}^{2}\right)\left(|g|_{2,-1, \Omega^{t}}^{2}+\|h(0)\|_{1,-1, \Omega}^{2}\right) \\
+c\left|F_{2}\right|_{2,-1, \Omega^{t}}^{2}+|\chi(0)|_{2,-1, \Omega}^{2} .
\end{gathered}
$$

To estimate the first factor in the first term on the r.h.s. of $(6.26)$ we use Lemma 5.5 which implies

$$
\begin{aligned}
\left\|w_{0}(t)\right\|_{1,0, \Omega}^{2} \leq & c \sup _{t^{\prime} \leq t}\left|w_{0}\left(t^{\prime}\right)\right|_{2,1, \Omega}^{2}+c e^{-t} \int_{0}^{t}\left|w_{0}\left(t^{\prime}\right)\right|_{2, \Omega}^{2} e^{t^{\prime}} d t^{\prime} \\
& +c \int_{0}^{t}\left(\left|q_{0}\left(t^{\prime}\right)\right|_{2,-1, \Omega}^{2}+\left|h_{0}\left(t^{\prime}\right)\right|_{2,-2, \Omega}^{2}+\left|f_{\varphi}\left(t^{\prime}\right)\right|_{2, \Omega}^{2}\right) d t^{\prime} \\
& +e^{-t}\left(\|w(0)\|_{1,0, \Omega}^{2}+|w(0)|_{2, S}^{2}\right)
\end{aligned}
$$

Using (6.15) in (6.27) yields

$$
\begin{aligned}
\left\|w_{0}(t)\right\|_{1,0, \Omega}^{2} \leq & c \sup _{t^{\prime} \leq t}\left|w_{0}\left(t^{\prime}\right)\right|_{2,1, \Omega}^{2}+c e^{-t} \int_{0}^{t}\left|w_{0}\left(t^{\prime}\right)\right|_{2, \Omega}^{2} e^{t^{\prime}} d t^{\prime} \\
& +c\left(|g|_{2,-1, \Omega^{t}}^{2}+\|h(0)\|_{1,-1, \Omega}^{2}+\left|f_{\varphi}\right|_{2, \Omega^{t}}^{2}\right) \\
& +c e^{-t}\left(\|w(0)\|_{1,0, \Omega}^{2}+|w(0)|_{2, S}^{2}\right) .
\end{aligned}
$$

To estimate the first term on the r.h.s. of (6.28) we use Lemma 5.6. From (5.23) and (5.24) we have

$$
\begin{aligned}
\left|w_{0}(t)\right|_{2,1, \Omega} & \leq|w(0)|_{2,1, \Omega}+\int_{0}^{t}\left(\left|q_{0}\left(t^{\prime}\right)\right|_{2, \Omega}+\left|h_{0}\left(t^{\prime}\right)\right|_{2,-1, \Omega}+\left|f_{\varphi}\left(t^{\prime}\right)\right|_{2, \Omega}\right) d t^{\prime} \\
& \leq|w(0)|_{2,1, \Omega}+t^{1 / 2}\left(|g|_{2,-1, \Omega^{t}}+\|h(0)\|_{1,-1, \Omega}+\left|f_{\varphi}\right|_{2, \Omega^{t}}\right) \\
& \leq c t^{1 / 2}\left(X_{1}^{\prime}+X_{2}^{\prime}\right), \quad t>1 .
\end{aligned}
$$


Moreover, from (5.6) we get the estimate

$$
\begin{aligned}
& \frac{1}{2}\left|w_{0}(t)\right|_{2, \Omega}^{2}+\nu\left|w_{0, x}\right|_{2, \Omega^{t}}^{2}+\frac{\nu}{2}\left|w_{0}\right|_{2,-1, \Omega^{t}}^{2}+\gamma\left|w_{0}\right|_{2, S^{t}}^{2} \\
& \quad \leq c\left(|g|_{2,-1, \Omega^{t}}^{2}+\|h(0)\|_{1,-1, \Omega}^{2}+\left|f_{\varphi}\right|_{2, \Omega^{t}}^{2}\right)+\nu \int_{S^{t}} \frac{a_{1}}{r} w_{0}^{2} d S+\frac{1}{2}|w(0)|_{2, \Omega}^{2} .
\end{aligned}
$$

REMARK. For domains close to a cylinder the boundary term on the r.h.s. can be absorbed by the second and third terms on the l.h.s.

Otherwise we consider (5.8) in the form

$$
\begin{aligned}
\frac{1}{2} \frac{d}{d t}\left|w_{0}\right|_{2, \Omega}^{2}+\nu\left|w_{0, x}\right|_{2, \Omega}^{2}+ & \frac{\nu}{2}\left|w_{0}\right|_{2,-1, \Omega}^{2}+\gamma\left|w_{0}\right|_{2, S}^{2} \\
& \leq \nu \int_{S} \frac{a_{1}}{r} w_{0}^{2} d S+c\left(\left|q_{0}\right|_{2, \Omega}^{2}+\left|h_{0}\right|_{2,-1, \Omega}^{2}+\left|f_{\varphi}\right|_{2, \Omega}^{2}\right) .
\end{aligned}
$$

Estimating the first term on the r.h.s. by some interpolation inequality we obtain

$$
\begin{aligned}
\frac{d}{d t}\left|w_{0}\right|_{2, \Omega}^{2}+\nu\left|w_{0, x}\right|_{2, \Omega}^{2}+\nu\left|w_{0}\right|_{2,-1, \Omega}^{2}+\gamma\left|w_{0}\right|_{2, S}^{2} \\
\quad \leq c_{1}\left|w_{0}\right|_{2, \Omega}^{2}+c\left(\left|q_{0}\right|_{2, \Omega}^{2}+\left|h_{0}\right|_{2,-1, \Omega}^{2}+\left|f_{\varphi}\right|_{2, \Omega}^{2}\right),
\end{aligned}
$$

where $c_{1}$ is a constant which follows from the interpolation inequality and depends on $a_{1} / r$.

From (6.32) we have

$$
\begin{aligned}
\frac{d}{d t}\left(\left|w_{0}(t)\right|_{2, \Omega}^{2} e^{-c_{1} t}\right)+\left(\nu\left|w_{0, x}\right|_{2, \Omega}^{2}\right. & \left.+\nu\left|w_{0}\right|_{2,-1, \Omega}^{2}+\gamma\left|w_{0}\right|_{2, S}^{2}\right) e^{-c_{1} t} \\
& \leq c e^{-c_{1} t}\left(\left|q_{0}(t)\right|_{2, \Omega}^{2}+\left|h_{0}(t)\right|_{2, \Omega}^{2}+\left|f_{\varphi}(t)\right|_{2, \Omega}^{2}\right) .
\end{aligned}
$$

Integrating (6.33) with respect to time yields

$$
\begin{aligned}
& \left|w_{0}(t)\right|_{2, \Omega}^{2}+e^{c_{1} t} \int_{0}^{t}\left(\nu\left|w_{0, x}\left(t^{\prime}\right)\right|_{2, \Omega}^{2}+\nu\left|w_{0}\left(t^{\prime}\right)\right|_{2,-1, \Omega}^{2}+\gamma\left|w_{0}\left(t^{\prime}\right)\right|_{2, S}^{2}\right) e^{-c_{1} t^{\prime}} d t^{\prime} \\
& \leq c e^{c_{1} t} \int_{0}^{t}\left(\left|q_{0}\left(t^{\prime}\right)\right|_{2, \Omega}^{2}+\left|h_{0}\left(t^{\prime}\right)\right|_{2,-1, \Omega}^{2}+\left|f_{\varphi}\left(t^{\prime}\right)\right|_{2, \Omega}^{2}\right) e^{-c_{1} t^{\prime}} d t^{\prime}+e^{c_{1} t}|w(0)|_{2, \Omega}^{2} .
\end{aligned}
$$

Since $e^{c_{1}\left(t-t^{\prime}\right)} \geq 1$ for $t \geq t^{\prime}$, using (6.15) we get

$$
\begin{aligned}
\left|w_{0}(t)\right|_{2, \Omega}^{2}+\nu\left|w_{0, x}\right|_{2, \Omega^{t}}^{2}+\nu\left|w_{0}\right|_{2,-1, \Omega^{t}}^{2}+\gamma\left|w_{0}\right|_{2, S^{t}}^{2} & \leq c e^{c_{1} t}\left(|g|_{2,-1, \Omega^{t}}^{2}+\|h(0)\|_{1,-1, \Omega}^{2}+\left|f_{\varphi}\right|_{2, \Omega^{t}}^{2}+|w(0)|_{2, \Omega}^{2}\right) \\
& \leq c e^{c_{1} t}\left(X_{1}^{\prime 2}+X_{2}^{\prime 2}\right) .
\end{aligned}
$$

Employing (6.29) and (6.35) in (6.27) yields

$$
\begin{aligned}
\left\|w_{0}(t)\right\|_{1,0, \Omega}^{2} \leq & |w(0)|_{2,1, \Omega}^{2}+\left|\int_{0}^{t}\left(\left|q_{0}\left(t^{\prime}\right)\right|_{2, \Omega}+\left|h_{0}\left(t^{\prime}\right)\right|_{2,-1, \Omega}+\left|f_{\varphi}\left(t^{\prime}\right)\right|_{2, \Omega}\right) d t^{\prime}\right|^{2} \\
& +c e^{c_{1} t}\left(|g|_{2,-1, \Omega^{t}}^{2}+\|h(0)\|_{1,-1, \Omega}^{2}+\left|f_{\varphi}\right|_{2, \Omega^{t}}^{2}\right) \\
& +e^{c_{1} t}|w(0)|_{2, \Omega}^{2}+e^{-t}\left(\|w(0)\|_{1,0, \Omega}^{2}+|w(0)|_{2, S}^{2}\right) \\
\leq & c e^{c t}\left(X_{1}^{\prime 2}+X_{2}^{\prime 2}\right)
\end{aligned}
$$

where (6.15) was also employed. 
Making use of (6.36) in (6.26) implies

$$
\begin{aligned}
\sup _{t}\left|\chi_{1} / r\right|_{2, \Omega}^{2}+\nu \int_{0}^{t} & \left\|\chi_{1} / r\right\|_{1, \Omega}^{2} d t^{\prime} \leq c\left[1+|w(0)|_{2,1, \Omega}^{2}\right. \\
& +e^{c_{1} t}\left(|g|_{2,-1, \Omega^{t}}+\|h(0)\|_{1,-1, \Omega}^{2}+\left|f_{\varphi}\right|_{2, \Omega^{t}}^{2}+|w(0)|_{2, \Omega}^{2}\right) \\
& \left.+e^{-t}\left(\|w(0)\|_{1, \Omega}^{2}+|w(0)|_{2, S}^{2}\right)\right]\left(|g|_{2,-1, \Omega^{t}}^{2}+\|h(0)\|_{1,-1, \Omega}^{2}\right) \\
& +c\left(\left|F_{2}\right|_{2,-1, \Omega^{t}}^{2}+|\chi(0)|_{2,-1, \Omega}^{2}\right) \\
\leq & c\left[1+e^{c_{1} t}\left(X_{1}^{\prime 2}+X_{2}^{\prime 2}\right)\right]\left(X_{1}^{\prime 2}+X_{2}^{\prime 2}\right)+c\left(Y_{1}^{\prime 2}+Y_{2}^{\prime 2}\right),
\end{aligned}
$$

where notation (6.22) was used.

For solutions of (6.10) we have

$$
\begin{aligned}
\left\|\chi_{1}\right\|_{2,1-\mu, \Omega^{t}} \leq & c\left|\chi_{1}\right|_{2,-(1+\mu), \Omega^{t}}+c\left|\frac{h_{0 \varphi}}{r} \chi_{1}\right|_{2,1-\mu, \Omega^{t}} \\
& +c\left|\frac{1}{r}\left(w_{0, z} h_{0 r}-w_{0, r} h_{0 z}+\frac{w_{0}}{r} h_{0 z}\right)\right|_{2,1-\mu, \Omega^{t}} \\
& +c\left|\frac{1}{r^{2}}\left(\frac{1}{r} h_{0 z, \varphi}-h_{0 \varphi, z}\right)\right|_{2,1-\mu, \Omega^{t}} \\
& +c\left|F_{2}\right|_{2,1-\mu, \Omega^{t}}+c\|\chi(0)\|_{1,1-\mu, \Omega} .
\end{aligned}
$$

The fourth term is dominated by

$$
c\left|\nabla h_{0}\right|_{2,-(1+\mu), \Omega^{t}} \leq c\left\|h_{0}\right\|_{2,-1, \Omega^{t}} .
$$

The third term on the r.h.s. of (6.38) is estimated by

$$
\begin{aligned}
\sup _{t}\left|w_{0, x}\right|_{2, \Omega}\left(\int_{0}^{t}\left|h_{0}\left(t^{\prime}\right)\right|_{\infty,-\mu, \Omega}^{2} d t^{\prime}\right)^{1 / 2}+\sup _{t}\left|w_{0} / r\right|_{2, \Omega}\left(\int_{0}^{t}\left|h_{0}\left(t^{\prime}\right)\right|_{\infty, 1-\mu, \Omega}^{2}\right)^{1 / 2} \\
\quad \leq c \sup _{t}\left\|w_{0}(t)\right\|_{1,0, \Omega}\left(\int_{0}^{t}\left\|h_{0}\left(t^{\prime}\right)\right\|_{2,-1, \Omega}^{2} d t^{\prime}\right)^{1 / 2} \\
\leq c \sup _{t}\left\|w_{0}(t)\right\|_{1,0, \Omega}\left\|h_{0}\right\|_{2,-1, \Omega^{t}} .
\end{aligned}
$$

The second term on the r.h.s. of (6.38) is bounded by

$$
\begin{aligned}
\left|\frac{h_{0 \varphi}}{r} \chi_{1}\right|_{2,1-\mu, \Omega^{t}} & =\left(\int_{0}^{t}\left|h_{0 \varphi} \frac{\chi_{1}}{r}\right|_{2,1-\mu, \Omega}^{2} d t^{\prime}\right)^{1 / 2} \\
& =\left(\int_{0}^{t} d t^{\prime} \int_{\Omega}\left|h_{0 \varphi}\right|^{2}\left|\frac{\chi_{1}}{r}\right|^{2} r^{2(1-\mu)} d x\right)^{1 / 2} \\
& \leq\left(\int_{0}^{t} d t^{\prime}\left|h_{0 \varphi}\left(t^{\prime}\right)\right|_{3,1-\mu, \Omega}^{2}\left|\frac{\chi_{1}}{r}\left(t^{\prime}\right)\right|_{6, \Omega}^{2}\right)^{1 / 2} \\
& \leq \sup _{t^{\prime} \leq t}\left|h_{0 \varphi}\left(t^{\prime}\right)\right|_{3,1-\mu, \Omega}\left(\int_{0}^{t}\left|\frac{\chi_{1}\left(t^{\prime}\right)}{r}\right|_{6, \Omega}^{2} d t^{\prime}\right)^{1 / 2} .
\end{aligned}
$$


Summarizing the above considerations gives

$$
\begin{aligned}
\left\|\chi_{1}\right\|_{2,1-\mu, \Omega^{t}} \leq & c\left|\chi_{1}\right|_{2,-(1+\mu), \Omega^{t}}+c \sup _{t^{\prime} \leq t}\left|h_{0 \varphi}\left(t^{\prime}\right)\right|_{3,1-\mu, \Omega}\left(\int_{0}^{t}\left\|\frac{\chi_{1}\left(t^{\prime}\right)}{r}\right\|_{1, \Omega}^{2} d t^{\prime}\right)^{1 / 2} \\
& +c \sup _{t}\left\|w_{0}\right\|_{1,0, \Omega}\left\|h_{0}\right\|_{2,-1, \Omega^{t}}+c\left\|h_{0}\right\|_{2,-1, \Omega^{t}} \\
& +c\left(\left|F_{2}\right|_{2,1-\mu, \Omega^{t}}+\|\chi(0)\|_{1,1-\mu, \Omega}\right) .
\end{aligned}
$$

In view of $(6.22)$ we have

$$
\begin{aligned}
\left|\chi_{1}\right|_{2,-(1+\mu), \Omega^{t}}+\left(\int_{0}^{t}\left\|\frac{\chi_{1}\left(t^{\prime}\right)}{r}\right\|_{1, \Omega}^{2} d t^{\prime}\right)^{1 / 2} & \\
& \leq c\left[1+e^{c t}\left(X_{1}^{\prime}+X_{2}^{\prime}\right)\right]\left(X_{1}^{\prime}+X_{2}^{\prime}\right)+c\left(Y_{1}^{\prime}+Y_{2}^{\prime}\right) .
\end{aligned}
$$

Applying (6.29), (6.36) and (6.40) in (6.39) yields

$$
\left\|\chi_{1}\right\|_{2,1-\mu, \Omega^{t}} \leq c\left(1+e^{c t} \widetilde{X}_{1}\right) t^{1 / 2} \widetilde{X}_{1}+c \widetilde{Y}_{1},
$$

where

$$
\widetilde{X}_{1}=X_{1}^{\prime}+X_{2}^{\prime}, \quad \widetilde{Y}_{1}=Y_{1}^{\prime}+Y_{2}^{\prime},
$$

and $t \geq t_{0}>0$.

In view of (6.41) we obtain from (6.23) the inequality

$$
\left\|\alpha^{\prime}\right\|_{2,2,1-\mu, \Omega^{t}} \leq c\left(e^{c t}+\widetilde{Y}_{1}\right) \widetilde{X}_{1}+c \widetilde{X}_{2},
$$

where $\widetilde{X}_{1} \leq 1$ and

$$
\widetilde{X}_{2}=X_{3}^{\prime}+X_{4}^{\prime}
$$

From (6.14), (6.41) and (6.42) we obtain

$$
K_{1}(t)=\left\|v_{1}\right\|_{A_{1-\mu}\left(\Omega^{t}\right)} \leq c\left(e^{c t} \widetilde{X}_{1}+\widetilde{Y}_{1}\right) t^{1 / 2} \widetilde{X}_{1}+c\left(e^{c t} \widetilde{X}_{1}+\widetilde{X}_{2}+\widetilde{Y}_{1}\right) .
$$

Therefore we can choose a constant $A$ such that the r.h.s. of (6.43) is bounded by $A$. This concludes the proof.

6.3. Estimate of the general step. Now we obtain a uniform bound for the constructed successive approximations.

Lemma 6.2. Assume that $g \in L_{2,-1}\left(\Omega^{T}\right), f_{\varphi} \in L_{2}\left(\Omega^{T}\right), f \in L_{2,1}\left(\Omega^{T}\right), F^{\prime} \in L_{2}\left(\Omega^{T}\right)$, $F_{1} \in L_{2,-\mu}\left(\Omega^{T}\right), F_{2} \in L_{2,-1}\left(\Omega^{T}\right), \mu \in(1 / 2,1), h(0) \in H_{-1}^{1}(\Omega), w(0) \in H_{0}^{1}(\Omega), v(0) \in$ $L_{2}(\Omega), \alpha^{\prime}(0) \in W_{2,1-\mu}^{1}(\Omega), \alpha_{1}(0) \in L_{2,-\mu}(\Omega), \chi(0) \in L_{2,-1}(\Omega) \cap H_{1-\mu}^{1}(\Omega)$. Assume that there exist two positive constants $A, B$ and a positive increasing function $\varphi_{1}$ such that

$$
\begin{aligned}
\varphi_{1}(A, B) e^{c t} \bar{X}(t) & \leq 1, \\
c\left(Y_{1}\left(Y_{1}+1\right)^{7 / 4}+Y_{1} Y_{2}+Y_{1}+Y_{2}\right) & \leq A, \\
c Y_{1} & \leq B,
\end{aligned}
$$

and assume that for some $\delta \geq 1, \delta_{0}=\|k-\gamma /(2 \nu)\|_{3 / 2,4,-\delta / 2, S}$ is so small that

$$
\delta_{0} \leq\left(c\left[Y_{1}\left(Y_{1}+1\right)^{7 / 4}+Y_{1} Y_{2}+Y_{1}+Y_{2}\right]\right)^{-1},
$$


where

$$
\begin{aligned}
\bar{X}(t)= & |g|_{2,1, \Omega^{t}}+|g|_{2,-1, \Omega^{t}}+\left|f_{\varphi}\right|_{2,-\mu, \Omega^{t}}+\left|F^{\prime}\right|_{2, \Omega^{t}} \\
& +\left|F_{1}\right|_{2,-\mu, \Omega^{t}}+\|h(0)\|_{1,-1, \Omega}+\|w(0)\|_{1,1-\mu, \Omega}+\|w(0)\|_{1,0, \Omega} \\
& +\left\|\left.\left|\alpha^{\prime}(0) \|_{1,2,1-\mu, \Omega}+\right| \alpha_{1}^{\prime}(0)\right|_{2,-\mu, \Omega},\right. \\
Y_{1}= & \left|F_{2}\right|_{2,-1, \Omega^{t}}+|\chi(0)|_{2,-1, \Omega}, \\
Y_{2}= & \left|F_{2}\right|_{2,1-\mu, \Omega^{t}}+\|\chi(0)\|_{1,1-\mu, \Omega}+\|v(0)\|_{2,2,1-\mu, \Omega} .
\end{aligned}
$$

Then

$$
\left\|v_{m}\right\|_{A_{1-\mu}\left(\Omega^{t}\right)} \leq A, \quad\left|\chi_{m} / r\right|_{\Omega^{t}} \leq B, \quad m \in \mathbb{N}, t \leq T,
$$

and $(6.44)_{1}^{\prime}$ implies the restriction on time:

$$
T \leq \frac{1}{c} \ln \frac{1}{\varphi_{1}(A, B) \bar{X}(T)}
$$

Proof. From (3.30) we have

$$
\left|h_{m}\right|_{2, \Omega}+\left(\int_{0}^{t}\left\|h_{m}\right\|_{1, \Omega}^{2} d t^{\prime}\right)^{1 / 2} \leq \varphi\left(t^{1 / 2} K_{m}(t)\right) X_{1},
$$

where

$$
X_{1}=|g|_{2,1, \Omega^{t}}+|h(0)|_{2, \Omega}, \quad K_{m}(t)=\left\|v_{m}\right\|_{A_{1-\mu}\left(\Omega^{t}\right)},
$$

and $\varphi$ will always denote an increasing positive function.

From (4.8) we get

$$
\left\|h_{m}\right\|_{2,-1, \Omega^{t}}+\left(\int_{0}^{t}\left\|q_{m}\right\|_{1,-1, \Omega}^{2} d t^{\prime}\right)^{1 / 2} \leq \varphi\left(t^{1 / 2} K_{m}(t), K_{m}(t)\right) X_{2},
$$

where

$$
X_{2}=X_{1}+|g|_{2,-1, \Omega^{t}}+\|h(0)\|_{1,-1, \Omega} .
$$

Assume that $\delta_{*}$ is sufficiently small and

$$
\sup _{t}\left|h_{m \varphi}\right|_{2,-1, \Omega} \leq \delta_{*} .
$$

Next Lemma 4.1 yields

$$
\begin{aligned}
\left|\chi_{m+1} / r\right|_{\Omega^{t}} \leq & c\left(\left\|h_{m}\right\|_{2,-1, \Omega^{t}}+\left|v_{m}\right|_{10 / 3, \Omega^{t}}+1\right) \delta_{0}\left(\varepsilon_{1} K_{m}(t)+c\left(1 / \varepsilon_{1}\right)\left|v_{m}\right|_{2, \Omega^{t}}\right) \\
& +c\left(1+\sup _{t}\left\|w_{m}\right\|_{1,0, \Omega}\right)\left\|h_{m}\right\|_{2,-1, \Omega^{t}} \\
& +c \sup _{t}\left\|w_{m}\right\|_{1,0, \Omega}\left|v_{m \varphi, z}\right|_{4,2,-3 / 4-\varepsilon, \Omega^{t}}+c Y_{1}
\end{aligned}
$$

for all $\varepsilon, \varepsilon_{1}>0$, where

$$
\delta_{0}=\|k-\gamma /(2 \nu)\|_{3 / 2,4,-\delta / 2, S}, \quad Y_{1}=|\chi(0)|_{2,-1, \Omega}+\left|F_{2}\right|_{2,-1, \Omega^{t}} .
$$

To estimate the norms involving $v_{m}$ we use (6.6) for step $m$. Then we have

$$
\left|v_{m}\right|_{\sigma, \Omega^{t}} \leq c\left|\alpha_{m}\right|_{\sigma, \Omega^{t}} \leq c\left(\left|\alpha_{m}^{\prime}\right|_{\sigma, \Omega^{t}}+\left|X_{m}\right|_{\sigma,-1, \Omega^{t}}\right)
$$

for $\sigma$ equal to either 2 or $10 / 3$, and the constant on the r.h.s. depends linearly on $R$. 
From Lemma 4.4 applied to problem (6.3) in step $m$ we have

$$
\begin{aligned}
\left|\alpha_{m}^{\prime}\right|_{\Omega^{t}}+\left|\alpha_{1 m}\right|_{2,-1, \Omega^{t}} \leq & \varphi\left(K_{m-1}\right)\left[\left|\chi_{m}\right|_{10 / 3, \Omega^{t}}\left\|h_{m-1}\right\|_{2,-1, \Omega^{t}}\right. \\
& \left.+\left\|h_{m-1}\right\|_{2,-1, \Omega^{t}}+\left\|w_{m-1}\right\|_{2,1-\mu, \Omega^{t}}+X_{3}\right],
\end{aligned}
$$

where

$$
X_{3}=\left|F^{\prime}\right|_{2, \Omega^{t}}+\left|\alpha^{\prime}(0)\right|_{2, \Omega} .
$$

In view of (6.54) and (6.55) we obtain from (6.52) the inequality

$$
\begin{aligned}
\left|\chi_{m+1} / r\right|_{\Omega^{t}} \leq & c\left[\left\|h_{m}\right\|_{2,-1, \Omega^{t}}+\left|\chi_{m}\right|_{10 / 3,-1, \Omega^{t}}\right. \\
& +\varphi\left(K_{m-1}\right)\left(\left|\chi_{m}\right|_{10 / 3, \Omega^{t}}\left\|h_{m-1}\right\|_{2,-1, \Omega^{t}}+\left\|h_{m-1}\right\|_{2,-1, \Omega^{t}}\right. \\
& \left.\left.+\left\|w_{m-1}\right\|_{2,1-\mu, \Omega^{t}}+X_{3}\right)+1\right] \delta_{0}\left[\varepsilon_{1} K_{m}+\left.\left.c \varepsilon_{1}^{-3 / 4}\right|_{m}\right|_{2,-1, \Omega^{t}}\right. \\
& +c \varepsilon_{1}^{-3 / 4} t^{1 / 2} \varphi\left(K_{m-1}\right)\left(\left|\chi_{m}\right|_{10 / 3, \Omega^{t}}\left\|h_{m-1}\right\|_{2,-1, \Omega^{t}}+\left\|h_{m-1}\right\|_{2,-1, \Omega^{t}}\right. \\
& \left.\left.+\left\|w_{m-1}\right\|_{2,1-\mu, \Omega^{t}}+X_{3}\right)\right]+c\left(1+\sup _{t}\left\|w_{m}\right\|_{1,0, \Omega}\right)\left\|h_{m}\right\|_{2,-1, \Omega^{t}} \\
& +c \sup _{t}\left\|w_{m}\right\|_{1,0, \Omega}\left|v_{m \varphi, z}\right|_{4,2,-3 / 4-\varepsilon, \Omega^{t}}+c Y_{1} .
\end{aligned}
$$

Introducing the notation

$$
L_{m}=\left|\chi_{m} / r\right|_{\Omega^{t}}
$$

and using (3.49) we obtain from (6.57) the inequality

$$
\begin{aligned}
L_{m+1} & \leq\left[L_{m}+1+\varphi\left(K_{m}, K_{m-1}\right)\left(L_{m}+1\right) X_{2}+\varphi\left(K_{m-1}\right)\left(\left\|w_{m-1}\right\|_{2,1-\mu, \Omega^{t}}\right.\right. \\
& \left.\left.+X_{3}\right)\right] \delta_{0}\left[\varepsilon_{1} K_{m}+c \varepsilon_{1}^{-3 / 4} L_{m}+c \varepsilon_{1}^{-3 / 4} t^{1 / 2} \varphi\left(K_{m-1}\right) .\right. \\
& \left.\cdot\left(\left(L_{m}+1\right) X_{2}+\left\|w_{m-1}\right\|_{2,1-\mu, \Omega^{t}}+X_{3}\right)\right]+c\left(1+\sup _{t}\left\|w_{m}\right\|_{1,0, \Omega}\right) \varphi\left(K_{m}\right) X_{2} \\
& +c \sup _{t}\left\|w_{m}\right\|_{1,0, \Omega}\left|v_{m \varphi, z}\right|_{4,2,-3 / 4-\varepsilon, \Omega^{t}}+c Y_{1} .
\end{aligned}
$$

Now we shall estimate the norms involving $w_{m-1}$ and $w_{m}$. Lemma 5.2 implies (6.60) $\left|w_{m}\right|_{2,-\mu, \Omega}+\left|w_{m, x}\right|_{2,-\mu, \Omega^{t}}+\left|w_{m}\right|_{2,-(1+\mu), \Omega^{t}}+\left|w_{m}\right|_{2,-\mu, S^{t}} \leq \varphi\left(K_{m}(t)\right) e^{c t} X_{4}$, where

$$
X_{4}=X_{2}+\left|f_{\varphi}\right|_{2,-\mu, \Omega^{t}}+|w(0)|_{2,-\mu, \Omega} .
$$

From Lemma 5.1 we have

$$
\left\|w_{m}\right\|_{2,1-\mu, \Omega^{t}} \leq \varphi\left(K_{m}(t)\right) X_{5}
$$

where

$$
X_{5}=X_{4}+\|w(0)\|_{1,1-\mu, \Omega} .
$$

From Lemma 5.5 we get

$$
\begin{aligned}
\left|w_{m, x}(t)\right|_{2, \Omega}+\left|w_{m}(t)\right|_{2,-1, \Omega} & +\left|w_{m}(t)\right|_{2, S} \\
& \leq \varphi\left(K_{m}(t)\right)\left[\sup _{t}\left|w_{m}(t)\right|_{2,1, \Omega}+\left|w_{m}\right|_{2, \Omega^{t}}+X_{6}\right],
\end{aligned}
$$

where

$$
X_{6}=X_{5}+\left|w_{, x}(0)\right|_{2, \Omega}+|w(0)|_{2,-1, \Omega}+|w(0)|_{2, S} .
$$


From Lemma 5.6 we obtain

$$
\left|w_{m}(t)\right|_{2,1, \Omega} \leq|w(0)|_{2,1, \Omega}+t^{1 / 2} X_{4} .
$$

Using (6.60) and (6.66) in (6.64) yields

$$
\left|w_{m, x}(t)\right|_{2, \Omega}+\left|w_{m}(t)\right|_{2,-1, \Omega}+\left|w_{m}(t)\right|_{2, S} \leq \varphi\left(K_{m}(t)\right) e^{c t} X_{6} .
$$

Employing (6.62) and (6.67) in (6.59) yields

$$
\begin{aligned}
L_{m+1} \leq & c\left[L_{m}+1+\varphi\left(K_{m}, K_{m-1}\right)\left(L_{m}+1\right) X_{7}\right] \delta_{0} \\
& \cdot\left[\varepsilon_{1} K_{m}+c \varepsilon_{1}^{-3 / 4} L_{m}+c \varepsilon_{1}^{-3 / 4} \varphi\left(K_{m-1}\right) t^{1 / 2}\left(L_{m}+1\right) X_{7}\right] \\
& +\varphi\left(K_{m}\right) e^{c t} X_{7}+\varphi\left(K_{m}\right) e^{c t} X_{7}\left|v_{m \varphi, z}\right|_{4,2,-3 / 4-\varepsilon, \Omega^{t}}+c Y_{1},
\end{aligned}
$$

where

$$
X_{7}=X_{2}+X_{3}+X_{5}+X_{6} .
$$

Now we shall estimate the last factor in the third term on the r.h.s. of (6.68). Using formula (4.51) applied to (6.6) for step $m$ we have

$$
v_{m \varphi, z}=-\alpha_{m 1}+\frac{1}{r} h_{m z} .
$$

Then applying (4.52) we have

$$
\left|v_{m \varphi, z}\right|_{4,2,-3 / 4-\varepsilon, \Omega^{t}} \leq c\left(\left|\alpha_{m 1}\right|_{4,2,-3 / 4-\varepsilon, \Omega^{t}}+\left\|h_{m}\right\|_{2,-1, \Omega^{t}}\right) .
$$

To estimate the first expression on the r.h.s. of (6.71) we apply (4.56) in the form

$$
\begin{aligned}
& \left(\int_{0}^{t}\left\|\alpha_{m 1}\left(t^{\prime}\right)\right\|_{1,-\mu, \Omega}^{2} d t^{\prime}\right)^{1 / 2} \leq \varphi\left(\left\|v_{m-1}\right\|_{A_{1-\mu}\left(\Omega^{t}\right)}\right) \\
& \cdot\left[\left|\chi_{m} / r\right|_{10 / 3, \Omega^{t}}\left\|h_{m-1}\right\|_{2,-1, \Omega^{t}}+\left\|h_{m-1}\right\|_{2,-1, \Omega^{t}}+\left\|w_{m-1}\right\|_{2,1-\mu, \Omega^{t}}+X_{8}\right],
\end{aligned}
$$

where

$$
X_{8}=\left|F^{\prime}\right|_{2, \Omega^{t}}+\left|F_{1}\right|_{2,-\mu, \Omega^{t}}+\left\|\left.\left|\alpha^{\prime}(0) \|_{1,2,1-\mu, \Omega}+\right| \alpha_{1}(0)\right|_{2,-\mu, \Omega} .\right.
$$

Using (6.47) and (6.62) in (6.72) implies

$$
\left(\int_{0}^{t}\left\|\alpha_{m 1}\left(t^{\prime}\right)\right\|_{1,-\mu, \Omega}^{2} d t^{\prime}\right)^{1 / 2} \leq \varphi\left(t^{1 / 2} K_{m-1}\right)\left[L_{m} X_{1}+X_{5}+X_{8}\right] .
$$

Taking $\mu=1 / 2+\varepsilon, \varepsilon>0$, we obtain from (6.71) and (6.74) the estimate

$$
\left|v_{m \varphi, z}\right|_{4,2,-3 / 4-\varepsilon, \Omega^{t}} \leq \varphi\left(t^{1 / 2} K_{m-1}\right)\left[\left|\chi_{m} / r\right|_{10 / 3, \Omega^{t}} X_{1}+X_{5}+X_{8}\right]+\varphi\left(K_{m}\right) X_{1} .
$$

Employing (6.75) in (6.68) yields

$$
\begin{aligned}
L_{m+1} \leq & c\left[L_{m}+1+\varphi\left(K_{m}, K_{m-1}\right)\left(L_{m}+1\right) X_{7}\right] \delta_{0} \\
& \cdot\left[\varepsilon_{1} K_{m}+c \varepsilon_{1}^{-3 / 4} L_{m}+c \varepsilon_{1}^{-3 / 4} \varphi\left(K_{m-1}\right) t^{1 / 2}\left(L_{m}+1\right) X_{7}\right] \\
& +\varphi\left(K_{m}, t^{1 / 2} K_{m-1}\right)\left(L_{m}+1\right) X_{9}+c Y_{1}
\end{aligned}
$$

where

$$
X_{9}=X_{1}+X_{5}+X_{7}+X_{8} .
$$


Lemma 4.3 implies

$$
\begin{aligned}
\left\|\alpha_{m+1}^{\prime}\right\|_{2,2,1-\mu, \Omega^{t}} \leq & \varphi\left(K_{m}\right) \sup _{t}\left|\alpha_{m+1}^{\prime}\right|_{2,1-\mu, \Omega}+L_{m+1} \varphi\left(K_{m}\right) X_{2} \\
& +\varphi\left(K_{m}\right) X_{5}+c\left|\alpha_{1 m+1}\right|_{2,-(1+\mu), \Omega^{t}}+c X_{8} .
\end{aligned}
$$

Lemma 4.4 yields

$$
\sup _{t}\left|\alpha_{m+1}^{\prime}\right|_{2, \Omega} \leq \varphi\left(K_{m}\right)\left[L_{m+1} X_{5}+X_{9}\right]
$$

and Lemma 4.5 gives

$$
\left|\alpha_{1 m+1}\right|_{2,-(1+\mu), \Omega^{t}} \leq \varphi\left(K_{m}\right)\left[L_{m+1} X_{5}+X_{9}\right] .
$$

From (6.78)-(6.80) we have

$$
\left\|\alpha_{m+1}^{\prime}\right\|_{2,2,1-\mu, \Omega^{t}} \leq \varphi\left(K_{m}\right)\left[L_{m+1} X_{5}+X_{9}\right] .
$$

Inequality (4.49) implies

(6.82) $\left\|\chi_{m+1}\right\|_{2,2,1-\mu, \Omega^{t}}$

$$
\begin{aligned}
\leq & {\left[\varepsilon_{3} K_{m}(t)+c\left(1 / \varepsilon_{3}\right)\left(\sup _{t}\left|v_{m}\right|_{2, \Omega}+\left|v_{m}\right|_{2, \Omega^{t}}+c\|v(0)\|_{2,2,1-\mu, \Omega}\right] L_{m+1}\right.} \\
& +c\left(1+L_{m+1}+\sup _{t}\left\|w_{m}\right\|_{1,0, \Omega}\right)\left\|h_{m}\right\|_{2,-1, \Omega^{t}} \\
& +c\left[\sup _{t}\left\|w_{m}\right\|_{1,0, \Omega} K_{m}+\left|\chi_{m+1}\right|_{2,-(1+\mu), \Omega^{t}}+\varepsilon_{4} K_{m}+c\left(1 / \varepsilon_{4}\right)\left|v_{m}\right|_{2, \Omega^{t}}+Y_{2}\right],
\end{aligned}
$$

where

$$
Y_{2}=\left|F_{2}\right|_{2,1-\mu, \Omega^{t}}+\|\chi(0)\|_{1,1-\mu, \Omega}+\|v(0)\|_{2,2,1-\mu, \Omega}
$$

Using (6.49) and (6.67) in (6.82) implies

$$
\begin{aligned}
\left\|\chi_{m+1}\right\|_{2,2,1-\mu, \Omega^{t}} \leq[ & \varepsilon_{3} K_{m}+c\left(1 / \varepsilon_{3}\right)\left(\sup _{t}\left|v_{m}\right|_{2, \Omega}+\left|v_{m}\right|_{2, \Omega^{t}}\right) \\
& \left.+c\|v(0)\|_{2,2,1-\mu, \Omega}\right] L_{m+1} \\
& +c L_{m+1} \varphi\left(K_{m}\right) X_{6}+\varphi\left(K_{m}\right) e^{c t} X_{6}+\left|\chi_{m+1}\right|_{2,-(1+\mu), \Omega^{t}} \\
& +\varepsilon_{4} K_{m}+c\left(1 / \varepsilon_{4}\right)\left|v_{m}\right|_{2, \Omega^{t}}+c Y_{2} .
\end{aligned}
$$

From (6.54) and (6.55) we have

$$
\begin{aligned}
& \sup _{t}\left|v_{m}\right|_{2, \Omega}+\left|v_{m}\right|_{2, \Omega^{t}} \leq c\left(t^{1 / 2}\left|\alpha_{m}^{\prime}\right|_{\Omega^{t}}+\left|\chi_{m} / r\right|_{\Omega^{t}}\right) \\
& \quad \leq \varphi\left(K_{m-1}\right) t^{1 / 2}\left[\left(L_{m}+1\right)\left\|h_{m-1}\right\|_{2,-1, \Omega^{t}}+\left\|w_{m-1}\right\|_{2,1-\mu, \Omega^{t}}+X_{3}\right]+c L_{m} .
\end{aligned}
$$

Using (6.49) and (6.62) in (6.85) yields

(6.86) $\sup _{t}\left|v_{m}\right|_{2, \Omega}+\left|v_{m}\right|_{2, \Omega^{t}} \leq \varphi\left(K_{m}, K_{m-1}\right)\left(L_{m}+1\right) t^{1 / 2}\left(X_{2}+X_{3}+X_{5}\right)+c L_{m}$.

Employing (6.86) in (6.84) gives

(6.87) $\left\|\chi_{m+1}\right\|_{2,2,1-\mu, \Omega^{t}} \leq\left[\varepsilon_{3} K_{m}+c \varepsilon_{3}^{-3 / 4}\left(\varphi\left(K_{m}, K_{m-1}\right)\left(L_{m}+1\right)\right.\right.$

$$
\left.\left.\cdot t^{1 / 2}\left(X_{2}+X_{3}+X_{5}\right)+c L_{m}\right)+c\|v(0)\|_{2,2,1-\mu, \Omega}\right] L_{m+1}
$$




$$
\begin{aligned}
& +L_{m+1} \varphi\left(K_{m}\right) X_{6}+\varphi\left(K_{m}\right) e^{c t} X_{6}+\left|\chi_{m+1}\right|_{2,-(1+\mu), \Omega^{t}} \\
& +\varepsilon_{4} K_{m}+c \varepsilon_{4}^{-3 / 4}\left(\varphi\left(K_{m}, K_{m-1}\right)\left(L_{m}+1\right) t^{1 / 2}\left(X_{2}+X_{3}+X_{5}\right)+c L_{m}\right)+c Y_{2} .
\end{aligned}
$$

From (6.81) and (6.87) we have

(6.88) $\left\|\alpha_{m+1}\right\|_{2,2,1-\mu, \Omega^{t}} \leq\left[\varepsilon_{5} K_{m}+c \varepsilon_{5}^{-3 / 4}\left(\varphi\left(K_{m}, K_{m-1}\right)\left(L_{m}+1\right)\right.\right.$

$$
\begin{aligned}
& \left.\left.\cdot t^{1 / 2}\left(X_{2}+X_{3}+X_{5}\right)+c L_{m}\right)+c\|v(0)\|_{2,2,1-\mu, \Omega}\right]\left(L_{m+1}+1\right) \\
& +\varphi\left(K_{m}\right)\left(L_{m+1}+e^{c t}\right)\left(X_{6}+X_{9}\right)+c\left|\chi_{m+1}\right|_{2,-(1+\mu), \Omega^{t}}+c Y_{2} .
\end{aligned}
$$

Applying [zaj 2] to (6.6) yields

$$
\left\|v_{m+1}\right\|_{A_{1-\mu}\left(\Omega^{t}\right)} \leq c\left\|\alpha_{m+1}\right\|_{2,2,1-\mu, \Omega^{t}}
$$

Hence (6.88) and (6.89) imply

$$
\begin{aligned}
K_{m+1} \leq & {\left[\varepsilon_{5} K_{m}+c \varepsilon_{5}^{-3 / 4}\left(\varphi\left(K_{m}, K_{m-1}\right)\left(L_{m}+1\right) t^{1 / 2} \bar{X}+c L_{m}\right)\right.} \\
& \left.+c\|v(0)\|_{2,2,1-\mu, \Omega}\right]\left(L_{m+1}+1\right) \\
& +\varphi\left(K_{m}\right)\left(L_{m+1}+e^{c t}\right) \bar{X}+c L_{m+1}+c Y_{2},
\end{aligned}
$$

where

$$
\bar{X}=\sum_{i=1}^{9} X_{i} .
$$

Assume that $A$ and $B$ are constants and

$$
L_{m} \leq B, \quad K_{m} \leq A, \quad K_{m-1} \leq A .
$$

Then (6.76) and (6.90) imply

$$
\begin{aligned}
L_{m+1} \leq & c[B+1+\varphi(A)(B+1) \bar{X}] \delta_{0}\left[\varepsilon_{1} A+c \varepsilon_{1}^{-3 / 4} B\right. \\
& \left.+c \varepsilon_{1}^{-3 / 4} \varphi(A) t^{1 / 2}(B+1) \bar{X}\right]+\varphi(A)(B+1) \bar{X}+c Y_{1}
\end{aligned}
$$

and

$$
\begin{aligned}
K_{m+1} \leq & {\left[\varepsilon_{5} A+c \varepsilon_{5}^{-3 / 4}\left(\varphi(A)(B+1) t^{1 / 2} \bar{X}+c B\right)\right.} \\
& \left.+c\|v(0)\|_{2,2,1-\mu, \Omega}\right](B+1)+\varphi(A)\left(B+e^{c t}\right) \bar{X}+c B+c Y_{2} .
\end{aligned}
$$

To show that $K_{m+1} \leq A$ and $L_{m+1} \leq B$ we assume that

$$
\begin{aligned}
{\left[\varepsilon_{5} A+c \varepsilon_{5}^{-3 / 4}\left(\varphi(A)(B+1) t^{1 / 2} \bar{X}+c B\right)+\right.} & \left.c\|v(0)\|_{2,2,1-\mu, \Omega}\right](B+1) \\
& +\varphi(A)\left(B+e^{c t}\right) \bar{X}+c B+c Y_{2} \leq A
\end{aligned}
$$

and

$$
\begin{aligned}
c[B+1+\varphi(A)(B+1) \bar{X}] \delta_{0}\left[\varepsilon_{1} A+c \varepsilon_{1}^{-3 / 4} B+c \varepsilon_{1}^{-3 / 4} \varphi(A) t^{1 / 2}(B+1) \bar{X}\right] & \\
& +\varphi(A)(B+1) \bar{X}+c Y_{1} \leq B .
\end{aligned}
$$

To have (6.96) we require that

$$
c(B+1)\left(\varepsilon_{1} A+c \varepsilon_{1}^{-3 / 4} B\right) \delta_{0}+c Y_{1} \leq \frac{1}{2} B, \quad \varphi(A, B) t^{1 / 2} \bar{X} \leq \frac{1}{2} B .
$$


and to have (6.95) we introduce the restrictions

$$
\begin{aligned}
& \varepsilon_{5}(B+1) \leq \frac{1}{3}, \\
& c(3(B+1))^{3 / 4} B(B+1)+c\|v(0)\|_{2,2,1-\mu, \Omega}(B+1)+c\left(B+Y_{2}\right) \leq \frac{1}{3} A, \\
& c(3(B+1))^{3 / 4} \varphi(A, B) e^{c t} \bar{X} \leq \frac{1}{3} A .
\end{aligned}
$$

Hence we obtain

$$
\begin{aligned}
& \delta_{0} \leq \frac{B}{c(B+1)(A+B)} \leq \frac{1}{c(A+B)}, \\
& B \geq Y_{1}, \\
& c B(B+1)^{7 / 4}+\|v(0)\|_{2,2,1-\mu, \Omega}(B+1)+c\left(B+Y_{2}\right) \leq \frac{1}{3} A .
\end{aligned}
$$

Continuing, we have

$$
\begin{aligned}
& B \geq Y_{1}, \\
& \frac{1}{3} A \geq c Y_{1}\left(Y_{1}+1\right)^{7 / 4}+\left(Y_{1}+1\right)\|v(0)\|_{2,2,1-\mu, \Omega}+c\left(Y_{1}+Y_{2}\right), \\
& \delta_{0} \leq \frac{1}{c\left[Y_{1}\left(Y_{1}+1\right)^{7 / 4}+\left(Y_{1}+1\right)\|v(0)\|_{2,2,1-\mu, \Omega}+Y_{1}+Y_{2}\right]}
\end{aligned}
$$

From the above considerations we obtain (6.45). This concludes the proof. 


\section{Local existence: convergence of the approximating sequence}

In this section we prove that the sequence examined in Section 6 converges. We show this step by step by dividing the interval $[0, T]$ into sufficiently small subintervals and by assuming that $\bar{X}$ and $\delta_{0}$ (see Lemma 6.2 ) are sufficiently small.

7.1. Problems for differences. To show convergence of the sequence constructed in Section 6 we introduce the differences

$$
\begin{array}{lll}
H_{m}=h_{m}-h_{m-1}, & Q_{m}=q_{m}-q_{m-1}, & W_{m}=w_{m}-w_{m-1}, \\
P_{m}=p_{m}-p_{m-1}, & A_{m}=\alpha_{m}-\alpha_{m-1}, & V_{m}=v_{m}-v_{m-1}, \\
K_{m}=\chi_{m}-\chi_{m-1} . &
\end{array}
$$

Now we formulate problems for the differences. From (6.1) we have

$$
\begin{aligned}
& H_{m, t}-\nu \operatorname{div} \mathbb{D}\left(H_{m}\right)+\nabla Q_{m}=-\left(V_{m} \cdot \nabla h_{m}+v_{m-1} \cdot \nabla H_{m}\right. \\
& \left.+H_{m} \cdot \nabla v_{m}+h_{m-1} \cdot \nabla V_{m}\right) \text {, } \\
& \operatorname{div} H_{m}=0 \text {, } \\
& H_{m} \cdot \bar{n}=0 \\
& \bar{n} \cdot \mathbb{D}\left(H_{m}\right) \cdot \bar{\tau}_{\alpha}+\frac{\gamma}{\nu} H_{m} \cdot \bar{\tau}_{\alpha}=0, \quad \alpha=1,2, \\
& \left.H_{m}\right|_{t=0}=0 .
\end{aligned}
$$

From (6.2) we get

$$
\begin{aligned}
& W_{m, t}+V_{m} \cdot \nabla w_{m}+v_{m-1} \cdot \nabla W_{m}+\frac{V_{m r}}{r} w_{m}+\frac{v_{m-1 r}}{r} W_{m} \\
& \quad-\nu \Delta W_{m}+\nu \frac{W_{m}}{r^{2}}=\frac{Q_{m}}{r}+\frac{2 \nu H_{m r}}{r^{2}}, \\
& a_{1} W_{m, r}+a_{2} W_{m, z}+\frac{\gamma}{\nu} W_{m}=\frac{1}{r} a_{1} W_{m}, \\
& \left.W_{m}\right|_{t=0}=0 .
\end{aligned}
$$

From (6.3) we obtain

$$
\begin{gathered}
A_{1 m+1, t}+V_{m} \cdot \nabla \alpha_{1 m}+v_{m} \cdot \nabla A_{1 m+1}-A_{1 m+1} v_{m r, r}-\alpha_{1 m} V_{m r, r} \\
-\frac{A_{2 m+1}}{r} h_{m r}-\frac{\alpha_{2 m}}{r} H_{m r}-A_{3 m+1} v_{m r, z}-\alpha_{3 m} V_{m r, z} \\
+\frac{2 \nu}{r^{2}}\left(H_{m r, z}-H_{m z, r}\right)+\frac{\nu}{r^{2}} A_{1 m+1}-\nu \Delta A_{1 m+1}=0,
\end{gathered}
$$


[cont.]

$$
\begin{aligned}
& A_{3 m+1, t}+V_{m} \cdot \nabla \alpha_{3 m}+v_{m} \cdot \nabla A_{3 m+1} \\
& \quad-A_{1 m+1} v_{m z, r}-\alpha_{1 m} V_{m z, r}-A_{3 m+1} v_{m z, z}-\alpha_{3 m} V_{m z, z} \\
& \quad-\frac{A_{2 m+1}}{r} h_{m z}-\frac{\alpha_{2 m}}{r} H_{m z}-\nu \Delta A_{3 m+1}=0, \\
& a_{2} A_{1 m+1}-a_{1} A_{3 m+1}=-\frac{2 a_{1}}{r} W_{m}+\frac{\gamma}{\nu} W_{m}, \\
& \left(a_{1} A_{1 m+1}+a_{2} A_{3 m+1}\right)_{, n}=\beta_{1} H_{m r}+\beta_{2} H_{m z}+\beta_{3} W_{m, r} \\
& \quad+\beta_{4} W_{m, z}+\beta_{5} W_{m}, \\
& \left.A_{1 m+1}\right|_{t=0}=0,\left.\quad A_{3 m+1}\right|_{t=0}=0 .
\end{aligned}
$$

From (6.4) we get

$$
\begin{aligned}
& K_{m+1, t}+v_{m} \cdot \nabla K_{m+1}+V_{m} \cdot \nabla \chi_{m}-\frac{h_{m \varphi}}{r} K_{m+1}-\frac{H_{m \varphi}}{r} \chi_{m} \\
& -\frac{v_{m r}}{r} K_{m+1}-\frac{V_{m r}}{r} \chi_{m}-\nu \Delta K_{m+1}+\nu \frac{K_{m+1}}{r^{2}} \\
& =-\frac{1}{r}\left(w_{m, z} H_{m r}+W_{m, z} h_{m-1 r}-w_{m, r} H_{m z}-W_{m, r} h_{m-1 z}\right. \\
& \left.+\frac{w_{m}}{r} H_{m z}+\frac{W_{m}}{r} h_{m-1 z}\right)+\frac{2 w_{m} V_{m \varphi, z}}{r}+\frac{2 W_{m} v_{m-1 \varphi, z}}{r} \\
& +\frac{2 \nu}{r^{2}}\left(\frac{1}{r} H_{m z, \varphi}-H_{m \varphi, z}\right) \\
& K_{m+1}=2(k-\gamma /(2 \nu)) V_{m} \cdot \bar{\tau}_{2} \\
& \left.K_{m+1}\right|_{t=0}=0
\end{aligned}
$$

Problem (6.5) gives

$$
\begin{aligned}
& \Delta P_{m}=-\nabla V_{m} \cdot \nabla v_{m}-\nabla v_{m-1} \cdot \nabla V_{m}, \\
& \frac{\partial P_{m}}{\partial n}=\nu \bar{n} \cdot \Delta V_{m}-\bar{n} \cdot V_{m} \cdot \nabla v_{m}-\bar{n} \cdot v_{m-1} \cdot \nabla V_{m} .
\end{aligned}
$$

Finally, problem (6.6) implies

$$
\begin{aligned}
& \operatorname{rot} V_{m+1}=A_{m+1}, \\
& \operatorname{div} V_{m+1}=0, \\
& V_{m+1} \cdot \bar{n}=0 .
\end{aligned}
$$

7.2. Estimates of differences. Now we obtain estimates for solutions of problems (7.2)-(7.7). First we obtain an estimate for $A_{m+1}$.

Lemma 7.1. Let the assumptions of Lemmas 6.1 and 6.2 hold. Then

$$
\begin{aligned}
\left\|A_{m+1}^{\prime}\right\|_{2,2,1-\mu, \Omega^{t}}+\left|A_{m+1}^{\prime}\right|_{2, \Omega} & +\left|A_{1 m+1}\right|_{2,-(1+\mu), \Omega^{t}} \\
\leq & \varphi(A)\left[\bar{X}\left(\sup _{t}\left|A_{2 m+1}\right|_{2,-1, \Omega}+\left\|V_{m}\right\|_{A_{1-\mu}\left(\Omega^{t}\right)}\right)\right. \\
& \left.+\left\|W_{m}\right\|_{2,1-\mu, \Omega^{t}}+\left\|H_{m}\right\|_{2,-1, \Omega^{t}}\right] .
\end{aligned}
$$


Proof. For solutions of (7.4) we have

$$
\begin{aligned}
\left\|A_{m+1}^{\prime}\right\|_{2,2,1-\mu, \Omega^{t}} & \leq c\left[\left|V_{m} \nabla \alpha_{m+1}^{\prime}\right|_{2,1-\mu, \Omega^{t}}+\left|v_{m-1} \nabla A_{m+1}^{\prime}\right|_{2,1-\mu, \Omega^{t}}\right. \\
+ & \left|A_{m+1}^{\prime} v_{m, x}\right|_{2,1-\mu, \Omega^{t}}+\left|\alpha_{m}^{\prime} V_{m, x}\right|_{2,1-\mu, \Omega^{t}}+\left|\frac{A_{2 m+1}}{r} h_{m}\right|_{2,1-\mu, \Omega^{t}} \\
+ & \left|\frac{\alpha_{2 m}}{r} H_{m}\right|_{2,1-\mu, \Omega^{t}}+\left|A_{1 m+1}\right|_{2,-(1+\mu), \Omega^{t}}+\left|H_{m, x}\right|_{2,-(1+\mu), \Omega^{t}} \\
+ & \left.\left\|W_{m}\right\|_{2,1-\mu, \Omega^{t}}+\left\|H_{m}\right\|_{1,1-\mu, \Omega^{t}}\right],
\end{aligned}
$$

where we assumed that $a_{1} \sim c r$ near $r=0$. Now we estimate the particular terms of the r.h.s. of (7.9). The first term is bounded by

$$
\sup _{t}\left|V_{m}\right|_{2 \lambda_{2}, \Omega}\left(\int_{0}^{t}\left|\nabla \alpha_{m+1}^{\prime}\left(t^{\prime}\right)\right|_{2 \lambda_{1}, 1-\mu, \Omega}^{2} d t^{\prime}\right)^{1 / 2} \equiv I_{1},
$$

whenever $1 / \lambda_{1}+1 / \lambda_{2}=1, \lambda_{1}<3$ but $\lambda_{1}$ is arbitrarily close to 3 . By Lemma 2.0 we have

$$
\begin{aligned}
I_{1} & \leq c t^{1 / 2}\left(\int_{0}^{t}\left|V_{m, t^{\prime}}\left(t^{\prime}\right)\right|_{2 \lambda_{2}, \Omega}^{2} d t^{\prime}\right)^{1 / 2}\left(\int_{0}^{t}\left\|\alpha_{m+1}^{\prime}\left(t^{\prime}\right)\right\|_{2,2,1-\mu, \Omega}^{2} d t^{\prime}\right)^{1 / 2} \\
& \leq c t^{1 / 2}\left(\int_{0}^{t}\left\|V_{m, t^{\prime}}\left(t^{\prime}\right)\right\|_{1,2,1-\mu, \Omega}^{2} d t^{\prime}\right)^{1 / 2}\left\|\alpha_{m+1}^{\prime}\right\|_{2,2,1-\mu, \Omega^{t}} \\
& \leq c t^{1 / 2}\left\|V_{m}\right\|_{A_{1-\mu}\left(\Omega^{t}\right)}\left\|\alpha_{m+1}^{\prime}\right\|_{2,2,1-\mu, \Omega^{t}} \leq c t^{1 / 2} \varphi(A)\left\|V_{m}\right\|_{A_{1-\mu}\left(\Omega^{t}\right)},
\end{aligned}
$$

where in the last inequality Lemma 6.2 was used.

The second and third terms on the r.h.s. of (7.9) are estimated by (see [zaj 5, Ch. 7])

$$
\varepsilon\left\|A_{m+1}^{\prime}\right\|_{2,2,1-\mu, \Omega^{t}}+c(1 / \varepsilon) t^{a} \varphi(A) \sup _{t}\left|A_{m+1}^{\prime}\right|_{2,1-\mu, \Omega}, \quad a>0 .
$$

The fourth term on the r.h.s. of (7.9) is bounded by

$$
\begin{aligned}
\sup _{t}\left|V_{m, x}\right|_{2,1-\mu, \Omega} & \left(\int_{0}^{t}\left|\alpha_{m}^{\prime}\left(t^{\prime}\right)\right|_{\infty, \Omega}^{2} d t^{\prime}\right)^{1 / 2} \\
& \leq c t^{1 / 2}\left(\int_{0}^{t}\left|V_{m, x t^{\prime}}\left(t^{\prime}\right)\right|_{2,1-\mu, \Omega}^{2} d t^{\prime}\right)^{1 / 2}\left(\int_{0}^{t}\left\|\alpha_{m}^{\prime}\left(t^{\prime}\right)\right\|_{2,2,1-\mu, \Omega} d t^{\prime}\right)^{1 / 2} \\
& \leq c t^{1 / 2}\left\|V_{m}\right\|_{A_{1-m \mu}\left(\Omega^{t}\right)}\left\|\alpha_{m}^{\prime}\right\|_{2,2,1-\mu, \Omega^{t}} \leq c t^{1 / 2} \varphi(A)\left\|V_{m}\right\|_{A_{1-\mu}\left(\Omega^{t}\right)}
\end{aligned}
$$

where we used Lemma 2.0 for $\mu>1 / 2$.

The fifth term is estimated by

$$
\begin{aligned}
& \sup _{t}\left|\frac{A_{2 m+1}}{r}\right|_{2, \Omega}\left(\int_{0}^{t}\left|h_{m}\left(t^{\prime}\right)\right|_{\infty, 1-\mu, \Omega}^{2} d t^{\prime}\right)^{1 / 2} \\
& \leq c\left\|h_{m}\right\|_{2,1-\mu, \Omega^{t}} \sup _{t}\left|\frac{A_{2 m+1}}{r}\right|_{2, \Omega} \leq c \varphi(A) \bar{X}(t) \sup _{t}\left|\frac{A_{2 m+1}}{r}\right|_{2, \Omega},
\end{aligned}
$$

where we used Lemma 6.2 and (6.47). 
We bound the sixth term by

$$
\begin{aligned}
\sup _{t}\left|\frac{\alpha_{2 m}}{r}\right|_{2, \Omega}\left(\int_{0}^{t}\left|H_{m}\left(t^{\prime}\right)\right|_{\infty, 1-\mu, \Omega}^{2} d t^{\prime}\right)^{1 / 2} & \leq \sup _{t}\left|\frac{\alpha_{2 m}}{r}\right|_{2, \Omega}\left\|H_{m}\right\|_{2,1-\mu, \Omega^{t}} \\
& \leq \varphi(B)\left\|H_{m}\right\|_{2,1-\mu, \Omega^{t}}
\end{aligned}
$$

Summarizing we obtain the estimate

$$
\begin{aligned}
\left\|A_{m+1}^{\prime}\right\|_{2,2,1-\mu, \Omega^{t}} & \leq t^{1 / 2} \varphi(A)\left\|V_{m}\right\|_{A_{1-\mu}\left(\Omega^{t}\right)}+t^{a} \varphi(A) \sup _{t}\left|A_{m+1}^{\prime}\right|_{2,1-\mu, \Omega} \\
& +\varphi(A) \bar{X} \sup _{t}\left|A_{2 m+1} / r\right|_{2, \Omega}+\varphi(B)\left\|H_{m}\right\|_{2,1-\mu, \Omega^{t}} \\
& +c\left(\left|A_{1 m+1}\right|_{2,-(1+\mu), \Omega^{t}}+\left|H_{m, x}\right|_{2,-(1+\mu), \Omega^{t}}+\left\|W_{m}\right\|_{2,1-\mu, \Omega^{t}}\right. \\
& \left.+\left\|H_{m}\right\|_{1,1-\mu, \Omega^{t}}\right) .
\end{aligned}
$$

Using

$$
\begin{aligned}
\sup _{t}\left|A_{m+1}^{\prime}\right|_{2,1-\mu, \Omega} & \leq t^{1 / 2}\left(\int_{0}^{t}\left|A_{m+1, t^{\prime}}^{\prime}\left(t^{\prime}\right)\right|_{2,1-\mu, \Omega}^{2} d t^{\prime}\right)^{1 / 2} \\
& \leq t^{1 / 2}\left\|A_{m+1}^{\prime}\right\|_{2,2,1-\mu, \Omega^{t}}
\end{aligned}
$$

in the r.h.s. of (7.10) and assuming that $t$ is sufficiently small we obtain from (7.10) the inequality

$$
\begin{aligned}
& \left\|A_{m+1}^{\prime}\right\|_{2,2,1-\mu, \Omega^{t}} \leq t^{1 / 2} \varphi(A)\left\|V_{m}\right\|_{A_{1-\mu}\left(\Omega^{t}\right)} \\
& \quad+\varphi(A) \bar{X} \sup _{t}\left|A_{2 m+1} / r\right|_{2, \Omega}+\varphi(B)\left\|H_{m}\right\|_{2,1-\mu, \Omega^{t}} \\
& \quad+c\left(\left|A_{1 m+1}\right|_{2,-(1+\mu), \Omega^{t}}+\left|H_{m, x}\right|_{2,-(1+\mu), \Omega^{t}}+\left\|W_{m}\right\|_{2,1-\mu, \Omega^{t}}+\left\|H_{m}\right\|_{1,1-\mu, \Omega^{t}}\right) .
\end{aligned}
$$

To obtain an estimate for $\left|A_{1 m+1}\right|_{2,-(1+\mu), \Omega^{t}}$ we introduce functions $\widetilde{A}_{1 m+1}, \widetilde{A}_{3 m+1}$ as solutions to the problem

$$
\begin{aligned}
& \widetilde{A}_{1 m+1, t}-\nu \Delta \widetilde{A}_{1 m+1}=0, \\
& \widetilde{A}_{3 m+1, t}-\nu \Delta \widetilde{A}_{3 m+1}=0, \\
& a_{2} \widetilde{A}_{1 m+1}-a_{1} \widetilde{A}_{3 m+1}=-\frac{2 a_{1}}{r} W_{m}+\frac{\gamma}{\nu} W_{m} \equiv G_{1 m}, \\
& \begin{aligned}
&\left(a_{1} \widetilde{A}_{1 m+1}+a_{2} \widetilde{A}_{3 m+1}\right)_{, n}=\beta_{1} H_{m r}+\beta_{2} H_{m z}+\beta_{3} W_{m, r} \\
&+\beta_{4} W_{m, z}+\beta_{5} W_{m} \equiv G_{2 m},
\end{aligned} \\
& \left.\widetilde{A}_{1 m+1}\right|_{t=0}=0, \\
& \left.\widetilde{A}_{3 m+1}\right|_{t=0}=0 .
\end{aligned}
$$

Then

$$
\bar{A}_{1 m+1}=A_{1 m+1}-\widetilde{A}_{1 m+1}, \quad \bar{A}_{3 m+1}=A_{3 m+1}-\widetilde{A}_{3 m+1}
$$

are solutions to the problem 


$$
\begin{aligned}
& \bar{A}_{1 m+1, t}-\nu \Delta \bar{A}_{1 m+1}+v_{m} \cdot \nabla \bar{A}_{1 m+1}+V_{m} \cdot \nabla \bar{\alpha}_{1 m} \\
& \quad-\left(\bar{A}_{1 m+1} v_{m r, r}+\bar{\alpha}_{1 m} V_{m r, r}+\bar{A}_{3 m+1} v_{m r, z}+\bar{\alpha}_{3 m} V_{m r, z}\right. \\
& \left.\quad+\frac{A_{2 m+1}}{r} h_{m r}+\frac{\alpha_{2 m}}{r} H_{m r}\right)+\frac{2 \nu}{r^{2}}\left(H_{m r, z}-H_{m z, r}\right)+\nu \frac{\bar{A}_{1 m+1}}{r^{2}} \\
& =-\left(v_{m} \cdot \nabla \widetilde{A}_{1 m+1}+V_{m} \cdot \nabla \widetilde{\alpha}_{1 m}\right)+\widetilde{A}_{1 m+1} v_{m r, r}+\widetilde{\alpha}_{1 m} V_{m r, r} \\
& \quad+\widetilde{A}_{3 m+1} v_{m r, z}+\widetilde{\alpha}_{3 m} V_{m r, z}-\nu \frac{\widetilde{A}_{1 m+1}}{r^{2}}, \\
& \bar{A}_{3 m+1, t}-\nu \Delta \bar{A}_{3 m+1}+v_{m} \cdot \nabla \bar{A}_{3 m+1}+V_{m} \cdot \nabla \bar{\alpha}_{3 m}-\bar{A}_{1 m+1} v_{m z, r} \\
& \quad-\bar{\alpha}_{1 m} V_{m z, r}-\bar{A}_{3 m+1} v_{m z, z}-\bar{\alpha}_{3 m} V_{m z, z}-\frac{A_{2 m+1}}{r} h_{m z}-\frac{\alpha_{2 m}}{r} H_{m z} \\
& =-v_{m} \cdot \nabla \widetilde{A}_{3 m+1}-V_{m} \cdot \nabla \widetilde{\alpha}_{3 m}+\widetilde{A}_{1 m+1} v_{m z, r}+\widetilde{\alpha}_{1 m} V_{m z, r} \\
& \quad+\widetilde{A}_{3 m+1} v_{m z, z}+\widetilde{\alpha}_{3 m} V_{m z, z}, \\
& a_{2} \bar{A}_{1 m+1}-a_{1} \bar{A}_{3 m+1}=0, \\
& \left(a_{1} \bar{A}_{1 m+1}+a_{2} \bar{A}_{3 m+1}\right)_{, n}=0 .
\end{aligned}
$$

First we obtain the energy estimate for solutions of problem (7.12). Multiplying (7.12) 1 by $\widetilde{A}_{1 m+1},(7.12)_{2}$ by $\widetilde{A}_{3 m+1}$, integrating the results over $\Omega$ and adding we have

$$
\frac{1}{2} \frac{d}{d t}\left|\widetilde{A}_{m+1}^{\prime}\right|_{2, \Omega}^{2}+\nu\left|\nabla \widetilde{A}_{m+1}^{\prime}\right|_{2, \Omega}^{2}=\nu \int_{S}\left(\widetilde{A}_{1 m+1, n} \widetilde{A}_{1 m+1}+\widetilde{A}_{3 m+1, n} \widetilde{A}_{3 m+1}\right) d S \equiv I,
$$

where $\widetilde{A}_{m+1}^{\prime}=\left(\widetilde{A}_{1 m+1}, \widetilde{A}_{3 m+1}\right)$.

Making use of the boundary conditions yields

$$
|I| \leq \varepsilon\left\|\widetilde{A}_{m+1}^{\prime}\right\|_{2,2,1-\mu, \Omega}^{2}+c(\varepsilon)\left|G_{m}^{\prime}\right|_{2, S}^{2},
$$

where $G_{m}^{\prime}=\left(G_{1 m}, G_{2 m}\right)$.

Applying potential theory to (7.12) implies

$$
\left\|\widetilde{A}_{m+1}^{\prime}\right\|_{2,2,1-\mu, \Omega^{t}} \leq c\left(\left\|G_{1 m}\right\|_{3 / 2,2,1-\mu, S^{t}}+\left\|G_{2 m}\right\|_{1 / 2,2,1-\mu, S^{t}}\right) .
$$

Integrating (7.15) with respect to time with the estimate for $I$, employing (7.16) and the explicit form of $G_{m}^{\prime}$ we obtain

$$
\left|\widetilde{A}_{m+1}^{\prime}\right|_{2, \Omega}+\left\|\widetilde{A}_{m+1}^{\prime}\right\|_{2,2,1-\mu, \Omega^{t}} \leq c\left(\left\|W_{m}\right\|_{2,1-\mu, \Omega^{t}}+\left\|H_{m}\right\|_{2,-1, \Omega^{t}}\right) .
$$

To estimate $\left|\widetilde{A}_{1 m+1}\right|_{2,-(1+\mu), \Omega^{t}}$ we consider the problem

$$
\begin{aligned}
& \widetilde{A}_{1 m+1, t}^{\prime}-\nu \Delta \widetilde{A}_{1 m+1}^{\prime}=-2 \nu \nabla \widetilde{A}_{1 m+1} \nabla \zeta-\nu \Delta \zeta \widetilde{A}_{1 m+1}, \\
& \left.\widetilde{A}_{1 m+1}^{\prime}\right|_{S_{2}}=\frac{a_{1}}{a_{2}} \widetilde{A}_{3 m+1}^{\prime}+\frac{1}{a_{2}} G_{1 m}^{\prime}, \\
& \left.\widetilde{A}_{1 m+1}\right|_{t=0}=0,
\end{aligned}
$$

where $\widetilde{A}_{1 m+1}^{\prime}=\widetilde{A}_{1 m+1} \zeta, \widetilde{A}_{3 m+1}^{\prime}=\widetilde{A}_{3 m+1} \zeta, G_{1 m}^{\prime}=G_{1 m} \zeta$, where $\zeta$ is defined before (4.33). 
Assuming $\left|a_{1} / a_{2}\right| \leq c r^{2},\left|\nabla\left(a_{1} / a_{2}\right)\right| \leq c r,\left|\nabla^{2}\left(a_{1} / a_{2}\right)\right| \leq c$ in a neighbourhood of the points $r=0, z \in\{-a, a\}$, we have

$$
\left\|\widetilde{A}_{1 m+1}\right\|_{2,1-\mu, \Omega^{t}} \leq c\left(\left\|\widetilde{A}_{3 m+1}\right\|_{2,2,1-\mu, \Omega^{t}}+\left\|W_{m}\right\|_{2,1-\mu, \Omega^{t}}+\left\|H_{m}\right\|_{2,-1, \Omega^{t}}\right) .
$$

By (7.17) and (7.19) we obtain

$$
\left|\widetilde{A}_{1 m+1}\right|_{2,-(1+\mu), \Omega^{t}} \leq c\left(\left\|W_{m}\right\|_{2,1-\mu, \Omega^{t}}+\left\|H_{m}\right\|_{2,-1, \Omega^{t}}\right) .
$$

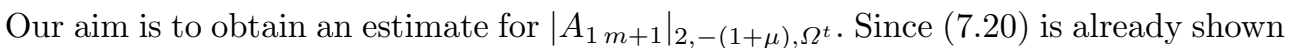
we need to find an estimate for $\left|\bar{A}_{1 m+1}\right|_{2,-(1+\mu), \Omega^{t}}$. For this purpose we use problem (7.14). Multiplying (7.14) 1 by $\bar{A}_{1 m+1} r^{-2 \mu}$ and integrating over $\Omega$ implies

$$
\begin{aligned}
& \frac{1}{2} \frac{d}{d t}\left|\bar{A}_{1 m+1}\right|_{2,-\mu, \Omega}^{2}-\nu \int_{\Omega} \Delta \bar{A}_{1 m+1} \bar{A}_{1 m+1} r^{-2 \mu} d x \\
& \quad+\int_{\Omega} v_{m} \cdot \nabla \bar{A}_{1 m+1} \bar{A}_{1 m+1} r^{-2 \mu} d x+\int_{\Omega} V_{m} \cdot \nabla \bar{\alpha}_{1 m} \bar{A}_{1 m} r^{-2 \mu} d x \\
& \quad-\int_{\Omega}\left(\bar{A}_{1 m+1} v_{m r, r}+\bar{\alpha}_{1 m} V_{m r, r}+\bar{A}_{3 m+1} v_{m r, z}+\bar{\alpha}_{3 m} V_{m r, z}\right. \\
& \left.\quad+\frac{A_{2 m+1}}{r} h_{m r}+\frac{\alpha_{2 m}}{r} H_{m r}\right) \bar{A}_{1 m+1} r^{-2 \mu} d x \\
& \quad+2 \nu \int_{\Omega} \frac{1}{r^{2}}\left(H_{m r, z}-H_{m z, r}\right) \bar{A}_{1 m+1} r^{-2 \mu} d x+\nu\left|\bar{A}_{1 m+1}\right|_{2,-(1+\mu), \Omega}^{2} \\
& =-\int_{\Omega}\left(v_{m} \cdot \nabla \widetilde{A}_{1 m+1}+V_{m} \cdot \nabla \widetilde{\alpha}_{1 m}\right) \bar{A}_{1 m+1} r^{-2 \mu} d x \\
& \quad+\int_{\Omega}\left(\widetilde{A}_{1 m+1} v_{m r, r}+\widetilde{\alpha}_{1 m} V_{m r, r}+\widetilde{A}_{3 m+1} v_{m r, z}+\widetilde{\alpha}_{3 m} V_{m r, z}\right) \bar{A}_{1 m+1} r^{-2 \mu} d x .
\end{aligned}
$$

To examine the second term on the l.h.s. of (7.21) we transform the boundary conditions $(7.14)_{3,4}$ to the form

$$
\begin{array}{ll}
\bar{A}_{1 m+1}=\frac{a_{1}}{a_{2}} \bar{A}_{3 m+1} & \text { for } a_{2} \neq 0, \\
\bar{A}_{1 m+1, n}=-\frac{a_{1, n}}{a_{1}} \bar{A}_{1 m+1}-\frac{a_{2, n}}{a_{1}} \bar{A}_{3 m+1}-\frac{a_{2}}{a_{1}} \bar{A}_{3 m+1, n} & \text { for } a_{1} \neq 0 .
\end{array}
$$

By integrating by parts, the second term on the l.h.s. of (7.21) takes the form

$$
\begin{aligned}
& -\nu \int_{\Omega} \operatorname{div}\left(\nabla \bar{A}_{1 m+1} \bar{A}_{1 m+1} r^{-2 \mu}\right) d x+\nu\left|\nabla \bar{A}_{1 m+1}\right|_{2,-\mu, \Omega}^{2} \\
& -2 \mu \nu \int_{\Omega} \nabla \bar{A}_{1 m+1} \bar{A}_{1 m+1} r^{-2 \mu-1} \nabla r d x,
\end{aligned}
$$

where by the Young inequality the last term is estimated by

$$
\frac{\nu}{2}\left|\bar{A}_{1 m+1, x}\right|_{2,-\mu, \Omega}^{2}+2 \mu^{2} \nu\left|\bar{A}_{1 m+1}\right|_{2,-(1+\mu), \Omega}^{2}
$$


and the first equals

$$
-\nu \int_{S} \bar{n} \cdot \nabla \bar{A}_{1 m+1} \bar{A}_{1 m+1} r^{-2 \mu} d S \equiv I .
$$

Hence, we have

$$
|I| \leq \varepsilon\left\|\bar{A}_{1 m+1}\right\|_{2,2,1-\mu, \Omega}^{2}+c(\varepsilon)\left|\bar{A}_{1 m+1}\right|_{2,1-\mu, \Omega}^{2},
$$

where we used Lemma 2.6.

The third term on the 1.h.s. of (7.21) equals

$$
\begin{aligned}
\frac{1}{2} \int_{\Omega} v_{m} \cdot \nabla \bar{A}_{1 m+1}^{2} r^{-2 \mu} & d x \\
& =\frac{1}{2} \int_{\Omega} v_{m} \cdot \nabla\left(\bar{A}_{1 m+1}^{2} r^{-2 \mu}\right) d x+\mu \int_{\Omega} \bar{A}_{1 m+1}^{2} r^{-2 \mu-1} v_{m} \cdot \nabla r d x
\end{aligned}
$$

where the first term vanishes and the second is estimated by

$$
\varepsilon\left|\bar{A}_{1 m+1}\right|_{2,-(1+\mu), \Omega}^{2}+c(\varepsilon) \int_{\Omega}\left|v_{m} \bar{A}_{1 m+1}\right|^{2} r^{-2 \mu} d x
$$

and we bound the last term by

$$
\left|v_{m}\right|_{\infty, \Omega}^{2}\left|\bar{A}_{1 m+1}\right|_{2,-\mu, \Omega}^{2} \leq c\left\|v_{m}\right\|_{3,2,1-\mu, \Omega}^{2}\left|\bar{A}_{1 m+1}\right|_{2,-\mu, \Omega}^{2}
$$

The fourth term on the l.h.s. of (7.21) is estimated by

$$
\begin{aligned}
\left|V_{m} \cdot \nabla \alpha_{1 m}^{\prime} r^{-\mu}\right|_{6 / 5, \Omega}\left|\bar{A}_{1 m+1}\right|_{6,-\mu, \Omega} \leq & \varepsilon\left\|\bar{A}_{1 m+1}\right\|_{1,-\mu, \Omega}^{2} \\
& +c(\varepsilon)\left|V_{m}\right|_{3, \Omega}^{2}\left|\nabla \alpha_{m}^{\prime}\right|_{2,-\mu, \Omega}^{2} .
\end{aligned}
$$

The fifth term on the l.h.s. of (7.21) is estimated by

$$
\begin{aligned}
\varepsilon\left\|\bar{A}_{m+1}^{\prime}\right\|_{1,-\mu, \Omega}^{2}+c(1 / \varepsilon)\left(\left|v_{m, x}\right|_{3, \Omega}^{2}\left|\bar{A}_{1 m+1}\right|_{2,-\mu, \Omega}^{2}+\left|\bar{\alpha}_{m}^{\prime}\right|_{2,-\mu, \Omega}^{2}\left|V_{m, x}\right|_{3, \Omega}^{2}\right. & \\
& \left.+\left|A_{2 m+1}\right|_{2,-1, \Omega}^{2}\left|h_{m}\right|_{3,-\mu, \Omega}^{2}+\left|\alpha_{2 m}\right|_{2,-1, \Omega}^{2}\left|H_{m}\right|_{3,-\mu, \Omega}^{2}\right) .
\end{aligned}
$$

We estimate the sixth term on the l.h.s. of (7.21) by

$$
\varepsilon\left|\bar{A}_{1 m+1}\right|_{2,-\mu, \Omega}^{2}+c(1 / \varepsilon)\left|H_{m, x}\right|_{2,-(1+\mu), \Omega}^{2} .
$$

The first term on the r.h.s. is bounded by

$$
\varepsilon\left\|\bar{A}_{1 m+1}\right\|_{1,-\mu, \Omega}^{2}+c(1 / \varepsilon)\left(\left|v_{m}\right|_{3, \Omega}^{2}\left|\nabla \widetilde{A}_{1 m+1}\right|_{2,-\mu, \Omega}^{2}+\left|V_{m}\right|_{3, \Omega}^{2}\left|\nabla \widetilde{\alpha}_{1 m}\right|_{2,-\mu, \Omega}^{2}\right) .
$$

Finally, the last integral on the r.h.s. of (7.21) is estimated by

$$
\varepsilon\left\|\bar{A}_{1 m+1}\right\|_{1,-\mu, \Omega}^{2}+c(1 / \varepsilon)\left(\left|v_{m, x}\right|_{3,-\mu, \Omega}^{2}\left|\widetilde{A}_{m+1}^{\prime}\right|_{2, \Omega}^{2}+\left|V_{m, x}\right|_{3,-\mu, \Omega}^{2}\left|\widetilde{\alpha}_{m}^{\prime}\right|_{2, \Omega}^{2}\right) .
$$

Employing the above estimates in (7.21) and integrating the result with respect to time we obtain 
$(7.23)$

$$
\begin{aligned}
& \left|\bar{A}_{1 m+1}\right|_{2,-\mu, \Omega}^{2}+\nu\left|\nabla \bar{A}_{1 m+1}\right|_{2,-\mu, \Omega^{t}}^{2}+\nu\left(1-2 \mu^{2}-\varepsilon\right)\left|\bar{A}_{1 m+1}\right|_{2,-(1+\mu), \Omega^{t}}^{2} \\
& \leq \quad \exp \left(\int_{0}^{t}\left(\left\|v_{m}\left(t^{\prime}\right)\right\|_{3,1-\mu, \Omega}^{2}+\left|v_{m, x}\right|_{3, \Omega}^{2}\right) d t^{\prime}\right)\left[\varepsilon\left\|\bar{A}_{m+1}^{\prime}\right\|_{2,2,1-\mu, \Omega^{t}}^{2}\right. \\
& \quad+c(1 / \varepsilon) \int_{0}^{t}\left\|\bar{A}_{m+1}^{\prime}\left(t^{\prime}\right)\right\|_{1, \Omega}^{2} d t^{\prime}+c\left(\sup _{t}\left|V_{m}\right|_{3, \Omega}^{2}\left|\nabla \alpha_{m}^{\prime}\right|_{2,-\mu, \Omega^{t}}^{2}\right. \\
& \quad+\sup _{t}\left|\bar{\alpha}_{m}^{\prime}\right|_{2,-\mu, \Omega}^{2} \int_{0}^{t}\left|V_{m, x}\right|_{3, \Omega}^{2} d t^{\prime}+\sup _{t}\left|A_{2 m+1}\right|_{2,-1, \Omega}^{2} \int_{0}^{t}\left|h_{m}\left(t^{\prime}\right)\right|_{3,-\mu, \Omega}^{2} d t^{\prime} \\
& \quad+\sup _{t}\left|\alpha_{2 m}\right|_{2,-1, \Omega}^{2} \int_{0}^{t}\left|H_{m}\left(t^{\prime}\right)\right|_{3,-\mu, \Omega}^{2} d t^{\prime}+\left|H_{m, x}\right|_{2,-(1+\mu), \Omega^{t}}^{2} \\
& \quad+\sup _{t}\left|v_{m}\right|_{3, \Omega}^{2}\left|\nabla \widetilde{A}_{1 m+1}\right|_{2,-\mu, \Omega^{t}}^{2}+\sup _{t}\left|V_{m}\right|_{3, \Omega}^{2}\left|\nabla \widetilde{\alpha}_{1 m}\right|_{2,-\mu, \Omega^{t}}^{2} \\
& \left.\left.\quad+\left|v_{m, x}\right|_{3,-\mu, \Omega^{t}}^{2} \sup _{t}\left|\widetilde{A}_{m+1}^{\prime}\right|_{2, \Omega}^{2}+\left|V_{m, x}\right|_{3,-\mu, \Omega^{t}}^{2} \sup _{t}\left|\widetilde{\alpha}_{m}^{\prime}\right|_{2, \Omega}^{2}\right)\right],
\end{aligned}
$$

where the exponent factor follows from integration of terms which contain $\left|\bar{A}_{1 m+1}\right|_{2,-\mu, \Omega}^{2}$.

Using the imbeddings

$$
\begin{gathered}
\sup _{t}\left|V_{m}\right|_{3, \Omega} \leq t^{1 / 2}\left(\int_{0}^{t}\left|V_{m, t^{\prime}}\right|_{3, \Omega}^{2} d t^{\prime}\right)^{1 / 2} \leq t^{1 / 2}\left(\int_{0}^{t}\left\|V_{m, t^{\prime}}\right\|_{1,1-\mu, \Omega}^{2} d t^{\prime}\right)^{1 / 2} \\
\int_{0}^{t}\left|V_{m, x}\left(t^{\prime}\right)\right|_{3, \Omega}^{2} d t^{\prime} \leq \int_{0}^{t}\left|V_{m, x x}\left(t^{\prime}\right)\right|_{3,1-\mu, \Omega}^{2} d t^{\prime} \leq \int_{0}^{t}\left\|V_{m, x}\left(t^{\prime}\right)\right\|_{2,2,1-\mu, \Omega}^{2} d t^{\prime} \\
\leq\left\|V_{m}\right\|_{A_{1-\mu}\left(\Omega^{t}\right)}^{2}, \\
\int_{0}^{t}\left\|\bar{A}_{m+1}^{\prime}\left(t^{\prime}\right)\right\|_{1, \Omega}^{2} d t^{\prime} \leq \varepsilon\left\|\bar{A}_{m+1}^{\prime}\right\|_{2,1-\mu, \Omega^{t}}^{2}+c(1 / \varepsilon)\left|\bar{A}_{m+1}^{\prime}\right|_{2, \Omega^{t}}^{2},
\end{gathered}
$$

estimate (6.45), estimates for small quantities bounded by $\bar{X}$ and (7.17) we obtain from (7.23) the inequality

$$
\begin{aligned}
\left|\bar{A}_{1 m+1}\right|_{2,-\mu, \Omega}^{2} & +\nu\left(1-\varepsilon_{*} / 2\right)\left|\nabla \bar{A}_{1 m+1}\right|_{2,-\mu, \Omega^{t}}^{2} \\
& +\nu\left(1-2 \mu^{2} / \varepsilon_{*}-\varepsilon_{0}\right)\left|\bar{A}_{1 m+1}\right|_{2,-(1+\mu), \Omega^{t}}^{2} \\
\leq & \varphi(A)\left[\varepsilon\left\|\bar{A}_{m+1}^{\prime}\right\|_{2,1-\mu, \Omega^{t}}^{2}+c(1 / \varepsilon)\left|\bar{A}_{m+1}^{\prime}\right|_{2, \Omega^{t}}^{2}\right. \\
& +\bar{X}^{2} \sup _{t}\left|A_{2 m+1}\right|_{2,-1, \Omega}^{2}+\bar{X}^{2}\left\|V_{m}\right\|_{A_{1-\mu}\left(\Omega^{t}\right)}^{2}+\left\|W_{m}\right\|_{2,1-\mu, \Omega^{t}}^{2} \\
& \left.+\left\|H_{m}\right\|_{2,-1, \Omega^{t}}^{2}+\left|H_{m, x}\right|_{2,-(1+\mu), \Omega^{t}}^{2}\right]
\end{aligned}
$$

where $\varepsilon_{0}$ is any small positive number, $\varepsilon_{*} \in(0,2)$.

Finally, we have to estimate the first term on the r.h.s. of (7.24). Applying potential theory to problem (7.14) yields 


$$
\begin{aligned}
\left\|\bar{A}_{m+1}^{\prime}\right\|_{2,2,1}-\mu, \Omega^{t} & \leq c\left[\left|v_{m} \cdot \nabla \bar{A}_{m+1}^{\prime}\right|_{2,1-\mu, \Omega^{t}}+\left|V_{m} \cdot \nabla \bar{\alpha}_{m}^{\prime}\right|_{2,1-\mu, \Omega^{t}}\right. \\
& +\left|\bar{A}_{m+1}^{\prime} \cdot v_{m, x}\right|_{2,1-\mu, \Omega^{t}}+\left|\bar{\alpha}_{m}^{\prime} \cdot V_{m, x}\right|_{2,1-\mu, \Omega^{t}}+\left|\frac{A_{2 m+1}}{r} h_{m}\right|_{2,1-\mu, \Omega^{t}} \\
& +\left|\frac{\alpha_{2 m}}{r} H_{m}\right|_{2,1-\mu, \Omega^{t}}+\left|\frac{1}{r^{2}} H_{m, x}\right|_{2,1-\mu, \Omega^{t}}+\left|\bar{A}_{1 m+1}\right|_{2,-(1+\mu), \Omega^{t}} \\
& +\left|v_{m} \cdot \nabla \widetilde{A}_{m+1}^{\prime}\right|_{2,1-\mu, \Omega^{t}}+\left|\widetilde{A}_{m+1}^{\prime} v_{m, x}\right|_{2,1-\mu, \Omega^{t}} \\
& \left.+\left|V_{m} \cdot \nabla \widetilde{\alpha}_{m}^{\prime}\right|_{2,1-\mu, \Omega^{t}}+\left|\widetilde{\alpha}_{m}^{\prime} V_{m, x}\right|_{2,1-\mu, \Omega^{t}}\right],
\end{aligned}
$$

where in the above estimate $v_{, x}$ replaces $v_{r, r}, v_{r, z}, v_{z, r}, v_{z, z}$ and similarly for other quantities.

Now we estimate the terms on the r.h.s. of (7.25). We estimate the first term by

$$
\left|v_{m}\right|_{\infty, \Omega^{t}}\left|\nabla \bar{A}_{m+1}^{\prime}\right|_{2,1-\mu, \Omega^{t}} \leq \varepsilon\left\|\bar{A}_{m+1}^{\prime}\right\|_{2,2,1-\mu, \Omega^{t}}+c(1 / \varepsilon) \varphi(A)\left|\bar{A}_{m+1}^{\prime}\right|_{2, \Omega^{t}},
$$

where we used the imbedding

$$
\left|v_{m}\right|_{\infty, \Omega^{t}} \leq\left|v_{m}\right|_{\infty, \Omega^{T}} \leq c\left\|v_{m}\right\|_{A_{1-\mu}\left(\Omega^{T}\right)} \leq c A
$$

here we exploit the fact that we examine convergence in a short time interval $(0, t)$ but quantities like $v_{m}, \alpha_{m}$ and so on are estimated on the large interval $[0, T]$ in view of Lemma 6.2. In this way we circumvent the difficulties connected with applying some imbeddings on small time intervals, which could imply that the imbedding constants behave as $t^{-a}, a>0$, for $t$ small.

The second term on the r.h.s. of (7.25) is estimated by

$$
\left(\int_{0}^{t}\left|V_{m}\right|_{3, \Omega}^{2}\left|\nabla \bar{\alpha}_{m}^{\prime}\right|_{6,1-\mu, \Omega}^{2} d t\right)^{1 / 2} \leq \sup _{t}\left|V_{m}\right|_{3, \Omega}\left\|\bar{\alpha}_{m}^{\prime}\right\|_{2,2,1-\mu, \Omega^{t}} \leq c t^{1 / 2}\left\|V_{m}\right\|_{A_{1-\mu}\left(\Omega^{t}\right)}^{2} \bar{X} .
$$

The third term is dominated by

$$
\begin{aligned}
\left(\int_{0}^{t}\left|\bar{A}_{m+1}^{\prime}\right|_{6^{\prime}, 1-\mu, \Omega}^{2}\left|v_{m, x}\right|_{3^{\prime}, \Omega}^{2} d t^{\prime}\right)^{1 / 2} & \leq \sup _{t}\left|A_{m+1}^{\prime}\right|_{6^{\prime}, 1-\mu, \Omega}\left(\int_{0}^{t}\left|v_{m, x}\right|_{3^{\prime}, \Omega}^{2} d t^{\prime}\right)^{1 / 2} \\
& \leq \sup _{t}\left\|\bar{A}_{m+1}^{\prime}\right\|_{1,2,1-\mu, \Omega}+\varphi(1 / \varepsilon, A) \sup _{t}\left|\bar{A}_{m+1}^{\prime}\right|_{2, \Omega} \\
& \leq \varepsilon\left\|\bar{A}_{m+1}^{\prime}\right\|_{2,2,1-\mu, \Omega^{t}}+\varphi(1 / \varepsilon, A) \sup _{t}\left|\bar{A}_{m+1}^{\prime}\right|_{2, \Omega},
\end{aligned}
$$

where $6^{\prime}<6$ and $3^{\prime}>3$ are such that $2 / 6^{\prime}+2 / 3^{\prime}=1$ and

$$
\left(\int_{0}^{t}\left|v_{m, x}\right|_{3^{\prime}, \Omega}^{2} d t^{\prime}\right)^{1 / 2} \leq c\left(\int_{0}^{t}\left\|v_{m}\right\|_{2,2,1-\mu, \Omega}^{2} d t^{\prime}\right)^{1 / 2} \leq c A
$$

and $\varphi(1 / \varepsilon, A)$ is an increasing positive function.

Similarly, the fourth term is bounded by

$$
\begin{aligned}
\sup _{t}\left|\bar{\alpha}_{m}^{\prime}\right|_{6^{\prime}, 1-\mu, \Omega}\left(\int_{0}^{t}\left|V_{m, x}\right|_{3^{\prime}, \Omega}^{2} d t^{\prime}\right)^{1 / 2} & \leq c\left\|\bar{\alpha}_{m}^{\prime}\right\|_{2,2,1-\mu, \Omega^{T}}\left\|V_{m}\right\|_{A_{1-\mu}\left(\Omega^{t}\right)} \\
& \leq c \bar{X}\left\|V_{m}\right\|_{A_{1-\mu}\left(\Omega^{t}\right)}
\end{aligned}
$$


The fifth term is estimated by

$$
\begin{aligned}
\sup _{t}\left|\frac{A_{2 m+1}}{r}\right|_{2, \Omega}\left(\int_{0}^{t}\left|h_{m}\right|_{\infty, 1-\mu, \Omega}^{2} d t^{\prime}\right)^{1 / 2} & \leq c \sup _{t}\left|A_{2 m+1}\right|_{2,-1, \Omega}\left\|h_{m}\right\|_{2,1-\mu, \Omega^{t}} \\
& \leq c \bar{X} \sup _{t}\left|A_{2 m+1}\right|_{2,-1, \Omega}
\end{aligned}
$$

and similarly the sixth by

$$
\sup _{t}\left|\alpha_{2 m}\right|_{2,-1, \Omega}\left\|H_{m}\right\|_{2,1-\mu, \Omega^{t}} \leq \varphi(A)\left\|H_{m}\right\|_{2,1-\mu, \Omega^{t}} .
$$

Finally, the last four terms on the r.h.s. of (7.25) are estimated in the following way:

$$
\begin{aligned}
\left|v_{m} \cdot \nabla \widetilde{A}_{m+1}^{\prime}\right|_{2,1-\mu, \Omega^{t}} & \leq c\left|v_{m}\right|_{\infty, \Omega^{T}}\left|\nabla \widetilde{A}_{m+1}^{\prime}\right|_{2,1-\mu, \Omega^{t}} \leq \varphi(A)\left\|\widetilde{A}_{m+1}^{\prime}\right\|_{2,2,1-\mu, \Omega^{t}}, \\
\left|\widetilde{A}_{m+1}^{\prime} v_{m, x}\right|_{2,1-\mu, \Omega^{t}} & \leq \sup _{t}\left|\widetilde{A}_{m+1}^{\prime}\right|_{6,1-\mu, \Omega}\left(\int_{0}^{T}\left|v_{m, x}\left(t^{\prime}\right)\right|_{3, \Omega}^{2} d t^{\prime}\right)^{1 / 2} \\
& \leq c\left\|\widetilde{A}_{m+1}^{\prime}\right\|_{2,2,1-\mu, \Omega^{t}}\left\|v_{m}\right\|_{A_{1-\mu}\left(\Omega^{T}\right)} \leq \varphi(A)\left\|\widetilde{A}_{m+1}^{\prime}\right\|_{2,2,1-\mu, \Omega^{t}}, \\
\left|V_{m} \cdot \nabla \widetilde{\alpha}_{m}^{\prime}\right|_{2,1-\mu, \Omega^{t}} & \leq \sup _{t}\left|V_{m}\right|_{3, \Omega}\left(\int_{0}^{t}\left|\nabla \widetilde{\alpha}_{m}^{\prime}\right|_{6,1-\mu, \Omega}^{2} d t^{\prime}\right)^{1 / 2} \\
& \leq c\left\|\widetilde{\alpha}_{m}^{\prime}\right\|_{2,1-\mu, \Omega^{t}} t^{1 / 2}\left\|V_{m}\right\|_{A_{1-\mu}\left(\Omega^{t}\right)} \leq c \bar{X} t^{1 / 2}\left\|V_{m}\right\|_{A_{1-\mu}\left(\Omega^{t}\right)}, \\
\left|\widetilde{\alpha}_{m}^{\prime} V_{m, x}\right|_{2,1-\mu, \Omega^{t}} & \leq \sup _{t}\left|\widetilde{\alpha}_{m}^{\prime}\right|_{6,1-\mu, \Omega}\left(\int_{0}^{t}\left|V_{m, x}\right|_{3, \Omega}^{2} d t^{\prime}\right)^{1 / 2} \\
& \leq c\left\|\widetilde{\alpha}_{m}^{\prime}\right\|_{2,2,1-\mu, \Omega^{t}}\left\|V_{m}\right\|_{A_{1-\mu}\left(\Omega^{t}\right)} \leq \varphi(A) \bar{X}\left\|V_{m}\right\|_{A_{1-\mu}\left(\Omega^{t}\right)} .
\end{aligned}
$$

Summarizing the above estimates we obtain

$$
\begin{aligned}
\left\|\bar{A}_{m+1}^{\prime}\right\|_{2,2,1-\mu, \Omega^{t}} \leq & \varphi(A)\left[\sup _{t}\left|\bar{A}_{m+1}^{\prime}\right|_{2, \Omega}+\left|\bar{A}_{m+1}^{\prime}\right|_{2, \Omega^{t}}\right. \\
& +\bar{X}\left(\left\|V_{m}\right\|_{A_{1-\mu}\left(\Omega^{t}\right)}+\sup _{t}\left|A_{2 m+1}\right|_{2,-1, \Omega}\right)+\left\|H_{m}\right\|_{2,-1, \Omega^{t}} \\
& \left.+\left\|W_{m}\right\|_{2,1-\mu, \Omega^{t}}+\left|\bar{A}_{1 m+1}\right|_{2,-(1+\mu), \Omega^{t}}\right],
\end{aligned}
$$

where (7.17) was exploited.

Finally, we have to estimate the second term on the r.h.s. of (7.24) and the first two terms on the r.h.s. of (7.26). For this purpose we multiply $(7.14)_{1}$ by $\bar{A}_{1 m+1}$ and $(7.14)_{2}$ by $\bar{A}_{3 m+1}$. Adding the results and integrating over $\Omega$ we obtain

$$
\begin{aligned}
\frac{d}{d t}\left|\bar{A}_{m+1}^{\prime}\right|_{2, \Omega}^{2}+\nu\left|\nabla \bar{A}_{m+1}^{\prime}\right|_{2, \Omega}^{2}+\nu\left|\bar{A}_{1 m+1}\right|_{2,-1, \Omega}^{2} \leq-\int_{\Omega} v_{m} \cdot \nabla \bar{A}_{m+1}^{\prime} \cdot \bar{A}_{m+1}^{\prime} d x \\
\quad-\int_{\Omega} V_{m} \cdot \nabla \bar{\alpha}_{m}^{\prime} \cdot \bar{A}_{m+1}^{\prime} d x+\int_{\Omega}\left(\left|\bar{A}_{m+1}^{\prime}\right|^{2}\left|v_{m, x}\right|+\left|\bar{\alpha}_{m}^{\prime}\right|\left|V_{m, x}\right|\left|\bar{A}_{m+1}^{\prime}\right|\right) d x \\
\quad+\int_{\Omega}\left(\left|\frac{A_{2 m+1}}{r}\right|\left|h_{m}\right|\left|\bar{A}_{m+1}^{\prime}\right|+\left|\frac{\alpha_{2 m}}{r}\right|\left|H_{m}\right|\left|\bar{A}_{m+1}^{\prime}\right|\right) d x
\end{aligned}
$$




$$
\begin{aligned}
& +\int_{\Omega}\left(\left|v_{m}\right|\left|\nabla \widetilde{A}_{m+1}^{\prime}\right|\left|\bar{A}_{m+1}^{\prime}\right|+\left|V_{m}\right|\left|\nabla \widetilde{\alpha}_{m}^{\prime}\right|\left|\bar{A}_{m+1}^{\prime}\right|\right) d x \\
& +\int_{\Omega}\left(\left|\widetilde{A}_{m+1}^{\prime}\right|\left|\bar{A}_{m+1}^{\prime}\right|\left|v_{m, x}\right|+\left|\widetilde{\alpha}_{m}^{\prime}\right|\left|V_{m, x}\right|\left|\bar{A}_{m+1}^{\prime}\right|\right) d x,
\end{aligned}
$$

where the first term on the r.h.s. vanishes because

$$
\int_{\Omega} \frac{1}{2} v_{m} \cdot \nabla\left|\bar{A}_{m+1}^{\prime}\right|^{2} d x=0 .
$$

Hence applying the Hölder and Young inequalities we obtain

$$
\begin{aligned}
\frac{d}{d t}\left|\bar{A}_{m+1}^{\prime}\right|_{2, \Omega}^{2} & +\nu\left|\nabla \bar{A}_{m+1}^{\prime}\right|_{2, \Omega}^{2}+\nu\left|\bar{A}_{1 m+1}\right|_{2,-1, \Omega}^{2} \leq \varepsilon\left|\bar{A}_{m+1}^{\prime}\right|_{6, \Omega}^{2} \\
& +c(\varepsilon)\left(\left|V_{m}\right|_{3, \Omega}^{2}\left|\nabla \bar{\alpha}_{m}^{\prime}\right|_{2, \Omega}^{2}+\left|\bar{A}_{m+1}^{\prime}\right|_{2, \Omega}^{2}\left|v_{m, x}\right|_{3, \Omega}^{2}+\left|\bar{\alpha}_{m}^{\prime}\right|_{2, \Omega}^{2}\left|V_{m, x}\right|_{3, \Omega}^{2}\right. \\
& +\left|A_{2 m+1}\right|_{2,-1, \Omega}^{2}\left|h_{m}\right|_{3, \Omega}^{2}+\left|\alpha_{2 m}\right|_{2,-1, \Omega}^{2}\left|H_{m}\right|_{3, \Omega}^{2} \\
& +\left|v_{m}\right|_{3, \Omega}^{2}\left|\nabla \widetilde{A}_{m+1}^{\prime}\right|_{2, \Omega}^{2}+\left|V_{m}\right|_{3, \Omega}^{2}\left|\nabla \widetilde{\alpha}_{m}^{\prime}\right|_{2, \Omega}^{2} \\
& \left.+\left|\widetilde{A}_{m+1}^{\prime}\right|_{2, \Omega}^{2}\left|v_{m, x}\right|_{3, \Omega}^{2}+\left|\widetilde{\alpha}_{m}^{\prime}\right|_{2, \Omega}^{2}\left|V_{m, x}\right|_{3, \Omega}^{2}\right) .
\end{aligned}
$$

Choosing $\varepsilon$ sufficiently small and integrating the result with respect to time we get

$$
\begin{aligned}
\left|\bar{A}_{m+1}^{\prime}\right|_{2, \Omega}^{2}+ & \nu\left|\nabla \bar{A}_{m+1}^{\prime}\right|_{2, \Omega^{t}}^{2}+\nu\left|\bar{A}_{1 m+1}^{\prime}\right|_{2,-1, \Omega^{t}}^{2} \\
\leq & \exp \left(\int_{0}^{t}\left|v_{m, x}\left(t^{\prime}\right)\right|_{3, \Omega}^{2} d t^{\prime}\right)\left[\varepsilon\left|\bar{A}_{3 m+1}\right|_{2, \Omega^{t}}^{2}+\sup _{t}\left|V_{m}\right|_{3, \Omega}^{2} \int_{0}^{t}\left|\nabla \bar{\alpha}_{m}^{\prime}\right|_{2, \Omega}^{2} d t^{\prime}\right. \\
& +\sup _{t}\left|\bar{\alpha}_{m}^{\prime}\right|_{2, \Omega}^{2} \int_{0}^{t}\left|V_{m, x}\right|_{3, \Omega}^{2} d t^{\prime}+\sup _{t}\left|A_{2} m+1\right|_{2,-1, \Omega}^{2} \int_{0}^{t}\left|h_{m}\right|_{3, \Omega}^{2} d t^{\prime} \\
& +\sup _{t}\left|\alpha_{2 m}\right|_{2,-1, \Omega}^{2} \int_{0}^{t}\left|H_{m}\right|_{3, \Omega}^{2} d t^{\prime}+\sup _{t}\left|v_{m}\right|_{3, \Omega}^{2} \int_{0}^{t}\left|\nabla \widetilde{A}_{m+1}^{\prime}\right|_{2, \Omega}^{2} d t^{\prime} \\
& +\sup _{t}\left|V_{m}\right|_{3, \Omega}^{2} \int_{0}^{t}\left|\nabla \widetilde{\alpha}_{m}^{\prime}\right|_{2, \Omega}^{2} d t^{\prime}+\sup _{t}\left|\widetilde{A}_{m+1}^{\prime}\right|_{2, \Omega}^{2} \int_{0}^{t}\left|v_{m, x}\right|_{3, \Omega}^{2} d t^{\prime} \\
& \left.+\sup _{t}\left|\widetilde{\alpha}^{\prime}\right|_{2, \Omega}^{2} \int_{0}^{t}\left|V_{m, x}\right|_{3, \Omega}^{2} d t^{\prime}\right] .
\end{aligned}
$$

Applying imbeddings $\left(7.23^{\prime}\right)$, Lemma 6.2 , estimates for small quantities bounded by $\bar{X}$ and (7.17) we obtain

$$
\begin{aligned}
\left|\bar{A}_{m+1}^{\prime}\right|_{2, \Omega}^{2}+ & \nu\left|\nabla \bar{A}_{m+1}^{\prime}\right|_{2, \Omega^{t}}^{2}+\nu\left|\bar{A}_{1 m+1}\right|_{2,-1, \Omega^{t}}^{2} \\
\leq & \varepsilon\left|\bar{A}_{3 m+1}\right|_{2, \Omega^{t}}^{2}+\varphi(A)\left[\bar{X}^{2}\left\|V_{m}\right\|_{A_{1-\mu}\left(\Omega^{t}\right)}^{2}+\bar{X}^{2} \sup _{t}\left|A_{2 m+1}\right|_{2,-1, \Omega}^{2}\right. \\
& \left.+\left\|H_{m}\right\|_{2,-1, \Omega^{t}}^{2}+\left\|W_{m}\right\|_{2,1-\mu, \Omega^{t}}^{2}\right] .
\end{aligned}
$$

Now we have to estimate the first term on the r.h.s. of (7.30). For this purpose we consider 
the boundary condition $(7.14)_{3}$ in the form

$$
\bar{A}_{3 m+1}=\frac{a_{2}}{a_{1}} \bar{A}_{1 m+1},
$$

so it is defined on $S_{1}$ only.

Let us introduce a new function $\widehat{A}_{3 m+1}$ by the relation

$$
\left.\widehat{A}_{3 m+1}\right|_{S_{1}}=\frac{a_{2}}{a_{1}} \bar{A}_{1 m+1} .
$$

Then the function

$$
A_{3 m+1}^{\prime \prime}=\bar{A}_{3 m+1}-\widehat{A}_{3 m+1}
$$

is such that

$$
\left.A_{3 m+1}^{\prime \prime}\right|_{S_{1}}=0 .
$$

Hence by the Poincaré inequality we have

$$
\begin{aligned}
\left|\bar{A}_{3 m+1}\right|_{2, \Omega} & \leq\left|\widehat{A}_{3 m+1}\right|_{2, \Omega}+\left|A_{3 m+1}^{\prime \prime}\right|_{2, \Omega} \\
& \leq c\left|\nabla A_{3 m+1}^{\prime \prime}\right|_{2, \Omega}+c\left|\widehat{A}_{3 m+1}\right|_{2, \Omega} \\
& \leq c\left|\nabla A_{3 m+1}^{\prime \prime}\right|_{2, \Omega}+c\left\|\widehat{A}_{3 m+1}\right\|_{1, \Omega} \\
& \leq c\left|\nabla \bar{A}_{3 m+1}\right|_{2, \Omega}+c\left\|\bar{A}_{1 m+1}\right\|_{1, \Omega} .
\end{aligned}
$$

In view of (7.33) the inequality (7.30) takes the form

$$
\begin{aligned}
\left|\bar{A}_{m+1}^{\prime}\right|_{2, \Omega}^{2}+\nu\left|\nabla \bar{A}_{m+1}\right|_{2, \Omega^{t}}^{2} & +\nu\left|\bar{A}_{1 m+1}\right|_{2,-1, \Omega^{t}}^{2} \\
\leq & \varphi(A)\left[\bar{X}^{2}\left\|V_{m}\right\|_{A_{1-\mu}\left(\Omega^{t}\right)}^{2}+\bar{X}^{2} \sup _{t}\left|A_{2 m+1}\right|_{2,-1, \Omega}^{2}\right. \\
& \left.+\left\|H_{m}\right\|_{2,-1, \Omega^{t}}^{2}+\left\|W_{m}\right\|_{2,1-\mu, \Omega^{t}}^{2}\right] .
\end{aligned}
$$

Using again (7.33) we obtain

$$
\begin{aligned}
\left|\bar{A}_{m+1}^{\prime}\right|_{2, \Omega}^{2}+\nu \int_{0}^{t}\left\|\bar{A}_{m+1}^{\prime}\right\|_{1, \Omega}^{2} d t^{\prime} & +\left|\bar{A}_{1 m+1}\right|_{2,-1, \Omega^{t}}^{2} \\
\leq & \varphi(A)\left[\bar{X}^{2}\left\|V_{m}\right\|_{A_{1-\mu}\left(\Omega^{t}\right)}^{2}+\bar{X}^{2} \sup _{t}\left|A_{2 m+1}\right|_{2,-1, \Omega}^{2}\right. \\
& \left.+\left\|H_{m}\right\|_{2,-1, \Omega^{t}}^{2}+\left\|W_{m}\right\|_{2,1-\mu, \Omega^{t}}^{2}\right] .
\end{aligned}
$$

From (7.24), (7.26) and (7.35) we have

$$
\begin{aligned}
\left|\bar{A}_{1 m+1}\right|_{2,-\mu, \Omega} & +\left|\nabla \bar{A}_{1 m+1}\right|_{2,-\mu, \Omega^{t}}+\left|\bar{A}_{1 m+1}\right|_{2,-(1+\mu), \Omega^{t}} \\
& +\left|A_{m+1}^{\prime}\right|_{2, \Omega}+\left(\int_{0}^{t}\left\|\bar{A}_{m+1}^{\prime}\left(t^{\prime}\right)\right\|_{1, \Omega}^{2} d t^{\prime}\right)^{1 / 2}+\left\|\bar{A}_{m+1}^{\prime}\right\|_{2,1-\mu, \Omega^{t}} \\
\leq & \varphi(A)\left[\bar{X}\left(\sup _{t}\left|A_{2 m+1}\right|_{2,-1, \Omega}+\left\|V_{m}\right\|_{A_{1-\mu}\left(\Omega^{t}\right)}\right)+\left\|W_{m}\right\|_{2,1-\mu, \Omega^{t}}\right. \\
& \left.+\left\|H_{m}\right\|_{2,-1, \Omega^{t}}\right] .
\end{aligned}
$$

Finally, from (7.17), (7.20) and (7.36) we obtain (7.8). This concludes the proof.

For solutions of problem (7.2) we have 
Lemma 7.2. Let the assumptions of Lemmas 6.1 and 6.2 hold. Then

$$
\left\|H_{m}\right\|_{2,-\mu^{\prime}, \Omega^{t}}+\left|\nabla Q_{m}\right|_{2,-\mu^{\prime}, \Omega^{t}} \leq c t^{1 / 2} \bar{X}\left\|V_{m}\right\|_{A_{1-\mu}\left(\Omega^{t}\right)}
$$

for all $\mu^{\prime} \in(0,1]$.

Proof. For solutions of problem (7.2) we have

$$
\begin{aligned}
& \left\|H_{m}\right\|_{2,-\mu^{\prime}, \Omega^{t}}+\left|\nabla Q_{m}\right|_{2,-\mu^{\prime}, \Omega^{t}} \leq c\left(\left|V_{m} \nabla h_{m}\right|_{2,-\mu^{\prime}, \Omega^{t}}\right. \\
& \left.\quad+\left|v_{m-1} \cdot \nabla H_{m}\right|_{2,-\mu^{\prime}, \Omega^{t}}+\left|H_{m} \cdot \nabla v_{m}\right|_{2,-\mu^{\prime}, \Omega^{t}}+\left|h_{m-1} \cdot \nabla V_{m}\right|_{2,-\mu^{\prime}, \Omega^{t}}\right)
\end{aligned}
$$

for all $\mu^{\prime} \in(0,1]$. We estimate the first term on the r.h.s. by

$$
\left|V_{m}\right|_{\infty, \Omega^{t}}\left|\nabla h_{m}\right|_{2,-\mu^{\prime}, \Omega^{t}} \leq c t^{1 / 2}\left\|V_{m}\right\|_{A_{1-\mu}\left(\Omega^{t}\right)}\left\|h_{m}\right\|_{2,-\mu^{\prime}, \Omega^{T}} \leq c t^{1 / 2} \bar{X}\left\|V_{m}\right\|_{A_{1-\mu}\left(\Omega^{t}\right)},
$$

the second term by

$$
\left|v_{m-1}\right|_{\infty, \Omega^{t}}\left|\nabla H_{m}\right|_{2,-\mu^{\prime}, \Omega^{t}} \leq c t^{1 / 2} \varphi(A)\left\|H_{m}\right\|_{2,-\mu^{\prime}, \Omega^{t}},
$$

and the third by

$$
\begin{aligned}
\sup _{t}\left|H_{m}\right|_{2,-\mu^{\prime}, \Omega}\left(\int_{0}^{T}\left|\nabla v_{m}\right|_{\infty, \Omega}^{2} d t^{\prime}\right)^{1 / 2} & \leq c t^{1 / 2}\left|H_{m, t}\right|_{2,-\mu^{\prime}, \Omega^{t}}\left\|v_{m}\right\|_{A_{1-\mu}\left(\Omega^{T}\right)} \\
& \leq c \varphi(A) t^{1 / 2}\left\|H_{m}\right\|_{2,-\mu^{\prime}, \Omega^{t}} .
\end{aligned}
$$

Finally, the last term on the r.h.s. of (7.38) is bounded by

$$
\begin{aligned}
& \left(\int_{0}^{t}\left|h_{m-1}\right|_{6,-\mu^{\prime}, \Omega}^{2}\left|V_{m, x}\right|_{3, \Omega}^{2} d t^{\prime}\right)^{1 / 2} \leq\left(\int_{0}^{t}\left|h_{m-1}\right|_{6,-\mu^{\prime}, \Omega}^{2}\left\|V_{m, x}\right\|_{1,1-\mu, \Omega}^{2} d t^{\prime}\right)^{1 / 2} \\
& \quad \leq \sup _{t}\left\|V_{m, x}\right\|_{1,1-\mu, \Omega}\left(\int_{0}^{t}\left|h_{m-1}\right|_{6,-\mu^{\prime}, \Omega}^{2} d t^{\prime}\right)^{1 / 2} \\
& \quad \leq c\left\|V_{m}\right\|_{A_{1-\mu}\left(\Omega^{t}\right)}\left(\int_{0}^{t}\left|h_{m-1, x x}\right|_{2,-\mu^{\prime}, \Omega}\left|h_{m-1}\right|_{2,-\mu^{\prime}, \Omega} d t^{\prime}\right)^{1 / 2} \\
& \quad \leq c\left\|V_{m}\right\|_{A_{1-\mu}\left(\Omega^{t}\right)} \sup _{t}\left|h_{m-1}\right|_{2,-\mu^{\prime}, \Omega}^{1 / 2} t^{1 / 2}\left\|h_{m-1}\right\|_{2,-\mu^{\prime}, \Omega^{t}}^{1 / 2} \\
& \quad \leq c t^{1 / 2}\left\|V_{m}\right\|_{A_{1-\mu}\left(\Omega^{t}\right)}\left(t^{1 / 2}\left|h_{m-1, t}\right|_{2,-\mu^{\prime}, \Omega^{t}}^{1 / 2}+|h(0)|_{2,-\mu^{\prime}, \Omega}^{1 / 2}\right)\left\|h_{m-1}\right\|_{2,-\mu^{\prime}, \Omega^{t}}^{1 / 2} \\
& \leq c t^{1 / 2}\left\|V_{m}\right\|_{A_{1-\mu}\left(\Omega^{t}\right)}\left(t^{1 / 2}\left\|h_{m-1}\right\|_{2,-\mu^{\prime}, \Omega^{t}}+|h(0)|_{2,-\mu^{\prime}, \Omega}\right) \leq c t^{1 / 2} \bar{X}\left\|V_{m}\right\|_{A_{1-\mu}\left(\Omega^{t}\right)} .
\end{aligned}
$$

Employing the above estimates in (7.38) and using the fact that $t$ is sufficiently small we obtain (7.37). This ends the proof.

For solutions of problem (7.3) we have

Lemma 7.3. Let the assumptions of Lemmas 6.1 and 6.2 hold. Then

$$
\begin{aligned}
\left|W_{m}\right|_{2,-\mu, \Omega}^{2}+\left(\int_{0}^{t}\left\|W_{m}\left(t^{\prime}\right)\right\|_{1,-\mu, \Omega}^{2} d t^{\prime}\right)^{1 / 2}+\left|W_{m}\right|_{2,-\mu, S^{t}}+\left\|W_{m}\right\|_{2,1-\mu, \Omega^{t}} \\
\quad \leq \varphi(A)\left[t^{a}\left\|V_{m}\right\|_{A_{1-\mu}\left(\Omega^{t}\right)}+\left|Q_{m}\right|_{2,-\mu, \Omega^{t}}+\left|H_{m}\right|_{2,-(1+\mu), \Omega^{t}}\right] .
\end{aligned}
$$


Proof. For solutions of problem (7.3) we obtain

$$
\begin{aligned}
\left\|W_{m}\right\|_{2,1-\mu, \Omega^{t}} \leq & c\left(\left|V_{m} \nabla w_{m}\right|_{2,1-\mu, \Omega^{t}}+\left|v_{m-1} \cdot \nabla W_{m}\right|_{2,1-\mu, \Omega^{t}}\right. \\
& +\left|\frac{V_{m r}}{r} w_{m}\right|_{2,1-\mu, \Omega^{t}}+\left|\frac{v_{m-1 r}}{r} W_{m}\right|_{2,1-\mu, \Omega^{t}} \\
& +\left|\frac{W_{m}}{r^{2}}\right|_{2,1-\mu, \Omega^{t}}+\left|\frac{Q_{m}}{r}\right|_{2,1-\mu, \Omega^{t}}+\left|\frac{H_{m}}{r^{2}}\right|_{2,1-\mu, \Omega^{t}} \\
& \left.+\left\|\frac{a_{1}}{r} W_{m}\right\|_{1 / 2,1-\mu, S^{t}}+\frac{\gamma}{\nu}\left\|W_{m}\right\|_{1 / 2,1-\mu, S^{t}}\right) .
\end{aligned}
$$

Now we estimate the particular terms on the r.h.s. of (7.40). We bound the first term by

$$
\begin{aligned}
& \left(\int_{0}^{t}\left|V_{m} \nabla w_{m}\right|_{2,1-\mu, \Omega}^{2} d t^{\prime}\right)^{1 / 2} \leq\left(\int_{0}^{t}\left|V_{m}\right|_{3, \Omega}^{2}\left|\nabla w_{m}\right|_{6,1-\mu, \Omega}^{2} d t^{\prime}\right)^{1 / 2} \\
& \leq \sup _{t}\left|V_{m}\right|_{3, \Omega}\left\|w_{m}\right\|_{2,1-\mu, \Omega^{t}} \leq c \sup _{t}\left\|V_{m}\right\|_{1,1-\mu, \Omega} \varphi(A) \bar{X} \\
& \leq c t^{1 / 2}\left(\int_{0}^{t}\left\|V_{m, t}\right\|_{1,1-\mu, \Omega}^{2} d t^{\prime}\right)^{1 / 2} \varphi(A) \bar{X} \leq c t^{1 / 2} \bar{X} \varphi(A)\left\|V_{m}\right\|_{A_{1-\mu}\left(\Omega^{t}\right)}
\end{aligned}
$$

the second term by

$$
\begin{aligned}
& \left(\int_{0}^{t}\left|v_{m-1} \cdot \nabla W_{m}\right|_{2,1-\mu, \Omega}^{2} d t^{\prime}\right)^{1 / 2} \leq\left(\int_{0}^{t}\left|v_{m-1}\right|_{4, \Omega}^{2}\left|\nabla W_{m}\right|_{4,1-\mu, \Omega}^{2} d t^{\prime}\right)^{1 / 2} \\
& \leq c \sup _{t}\left|v_{m-1}\right|_{4, \Omega}\left(\int_{0}^{t}\left|W_{m, x x}\right|_{2,1-\mu, \Omega}^{7 / 4}\left|W_{m}\right|_{2,1-\mu, \Omega}^{1 / 4} d t^{\prime}\right)^{1 / 2} \\
& \leq c \sup _{t}\left(\left|v_{m-1, x x}\right|_{2,1-\mu, \Omega}^{\theta_{1}}\left|v_{m-1}\right|_{2,1-\mu, \Omega}^{1-\theta_{1}}\right) \sup _{t}\left|W_{m}\right|_{2,1-\mu, \Omega}^{1 / 8}\left(\int_{0}^{t}\left|W_{m, x x}\right|_{2,1-\mu, \Omega}^{7 / 4} d t^{\prime}\right)^{1 / 2} \\
& \leq c t^{a}\left(\left\|v_{m-1, x}\right\|_{2,1-\mu, \Omega^{t}}+\|v(0)\|_{2,1-\mu, \Omega}\right)^{\theta_{1}}\left|v_{m-1, t}\right|_{2,1-\mu, \Omega^{t}}^{1-\theta_{1}} . \\
& \cdot\left|W_{m, t}\right|_{2,1-\mu, \Omega^{t}}^{1 / 8}\left\|W_{m}\right\|_{2,1-\mu, \Omega^{t}}^{7 / 8} \leq c t^{a} \varphi(A)\left\|W_{m}\right\|_{2,1-\mu, \Omega^{t}},
\end{aligned}
$$

the third term by

$$
\begin{aligned}
\left(\int_{0}^{t}\left|\frac{V_{m, r}}{r} w_{m}\right|_{2,1-\mu, \Omega}^{2} d t^{\prime}\right)^{1 / 2} \leq\left(\int_{0}^{t}\left|V_{m}\right|_{4, \Omega}^{2}\left|w_{m}\right|_{4,-\mu, \Omega}^{2} d t^{\prime}\right)^{1 / 2} \\
\quad \leq \sup _{t}\left|V_{m}\right|_{4, \Omega} \int_{0}^{t}\left|w_{m}\right|_{4,-\mu, \Omega}^{2} d t^{\prime} \\
\quad \leq \sup _{t}\left(\left|V_{m, x x}\right|_{2,1-\mu, \Omega}^{\theta_{1}}\left|V_{m}\right|_{2,1-\mu, \Omega}^{1-\theta_{1}}\right)\left(\int_{0}^{t}\left|w_{m, x x}\right|_{2,1-\mu, \Omega}^{2 \theta_{2}}\left|w_{m}\right|_{2,1-\mu, \Omega}^{2\left(1-\theta_{2}\right)} d t^{\prime}\right)^{1 / 2}
\end{aligned}
$$




$$
\begin{aligned}
& \leq c t^{a}\left\|V_{m, x}\right\|_{2,1-\mu, \Omega^{t}}^{\theta_{1}}\left|V_{m, t}\right|_{2,1-\mu, \Omega^{t}}^{1-\theta_{1}}\left|w_{m, t}\right|_{2,1-\mu, \Omega^{t}}^{1-\theta_{2}}\left(\int_{0}^{t}\left|w_{m, x x}\right|_{2,1-\mu, \Omega}^{2 \theta_{2}}\right)^{1 / 2} \\
& \leq c t^{a}\left\|V_{m}\right\|_{A_{1-\mu}\left(\Omega^{t}\right)}\left\|w_{m}\right\|_{2,1-\mu, \Omega^{t}} \leq c t^{a} \bar{X}\left\|V_{m}\right\|_{A_{1-\mu}\left(\Omega^{t}\right)}
\end{aligned}
$$

for some $a>0$, and the fourth term by

$$
\begin{gathered}
\left(\int_{0}^{t}\left|v_{m-1}\right|_{4, \Omega}^{2}\left|W_{m}\right|_{4,-\mu, \Omega}^{2} d t^{\prime}\right)^{1 / 2} \leq \sup _{t}\left|v_{m-1}\right|_{4, \Omega}\left(\int_{0}^{t}\left|W_{m}\right|_{4,-\mu, \Omega}^{2} d t^{\prime}\right)^{1 / 2} \\
\leq \sup _{t}\left(\left|v_{m-1, x x}\right|_{2,1-\mu, \Omega}^{\theta_{1}}\left|v_{m-1}\right|_{2,1-\mu, \Omega}^{1-\theta_{1}}\right)\left(\int_{0}^{t}\left|W_{m, x x}\right|_{2,1-\mu, \Omega}^{2 \theta_{2}}\left|W_{m}\right|_{2,1-\mu, \Omega}^{2\left(1-\theta_{2}\right)} d t^{\prime}\right)^{1 / 2} \\
\leq c t^{a}\left(\left\|v_{m-1, x}\right\|_{2,1-\mu, \Omega^{t}}+\left\|v_{m-1}(0)\right\|_{2,1-\mu, \Omega}\right) \\
\cdot\left|W_{m, t}\right|_{2,1-\mu, \Omega^{t}}^{1-\theta_{2}}\left\|W_{m}\right\|_{2,1-\mu, \Omega^{t}}^{\theta_{2}} \leq \varphi(A) t^{a}\left\|W_{m}\right\|_{2,1-\mu, \Omega^{t}} .
\end{gathered}
$$

To estimate the last but one term we assume that

$$
\left|\frac{a_{1}}{r}\right| \leq c, \quad\left|\left(\frac{a_{1}}{r}\right)_{, r}\right| \leq \frac{c}{r} .
$$

Then the term is bounded by

$$
\begin{aligned}
c\left(\left\|W_{m}\right\|_{1,1-\mu, \Omega^{t}}+\left|W_{m}\right|_{2,-\mu, \Omega^{t}}\right) & \leq c\left\|W_{m}\right\|_{1,1-\mu, \Omega^{t}} \\
& \leq \varepsilon\left\|W_{m}\right\|_{2,1-\mu, \Omega^{t}}+c(1 / \varepsilon)\left|W_{m}\right|_{2,1-\mu, \Omega^{t}} \\
& \leq \varepsilon\left\|W_{m}\right\|_{2,1-\mu, \Omega^{t}}+c(1 / \varepsilon) t^{a}\left|W_{m, t}\right|_{2,1-\mu, \Omega^{t}} \\
& \leq c t^{a}\left\|W_{m}\right\|_{2,1-\mu, \Omega^{t}},
\end{aligned}
$$

where $a>0$. The last term has the same bound.

Employing the above estimates and choosing $t$ sufficiently small we obtain from (7.40) the inequality

$$
\begin{aligned}
\left\|W_{m}\right\|_{2,1-\mu, \Omega^{t}} \leq & c t^{a} \varphi(A)\left\|V_{m}\right\|_{A_{1-\mu}\left(\Omega^{t}\right)}+c\left|W_{m}\right|_{2,-(1+\mu), \Omega^{t}} \\
& +\left|Q_{m}\right|_{2,-\mu, \Omega^{t}}+c\left|H_{m}\right|_{2,-(1+\mu), \Omega^{t}} .
\end{aligned}
$$

To close the estimate we have to find a bound for the second term on the r.h.s. of (7.41). For this purpose we multiply $(7.3)_{1}$ by $W_{m} r^{-2 \mu}$ and integrate over $\Omega$ to obtain

$$
\begin{aligned}
\frac{1}{2} \frac{d}{d t}\left|W_{m}\right|_{2,-\mu, \Omega}^{2}+\int_{\Omega} V_{m} \cdot \nabla & w_{m} W_{m} r^{-2 \mu} d x+\int_{\Omega} v_{m-1} \cdot \nabla W_{m} W_{m} r^{-2 \mu} d x \\
& +\int_{\Omega} \frac{V_{m r}}{r} w_{m} W_{m} r^{-2 \mu} d x+\int_{\Omega} \frac{v_{m-1 r}}{r} W_{m}^{2} r^{-2 \mu} d x \\
& -\nu \int_{\Omega} \Delta W_{m} W_{m} r^{-2 \mu} d x+\nu\left|W_{m}\right|_{2,-(1+\mu), \Omega}^{2} \\
= & \int_{\Omega} \frac{Q_{m}}{r} W_{m} r^{-2 \mu} d x+2 \nu \int_{\Omega} \frac{H_{m r}}{r^{2}} W_{m} r^{-2 \mu} d x
\end{aligned}
$$


The third term on the l.h.s. of (7.42) takes the form

$$
\begin{aligned}
\frac{1}{2} \int_{\Omega} v_{m-1} \cdot \nabla W_{m}^{2} r^{-2 \mu} d x & =\frac{1}{2} \int_{\Omega} v_{m-1} \cdot \nabla\left(W_{m}^{2} r^{-2 \mu}\right) d x+\mu \int_{\Omega} v_{m-1} \cdot \nabla r r^{-2 \mu-1} W_{m}^{2} d x \\
& =\mu \int_{\Omega} v_{m-1} r^{-2 \mu-1} W_{m}^{2} d x \equiv I_{1} .
\end{aligned}
$$

By the Hölder and Young inequalities we have

$$
\left|I_{1}\right| \leq \varepsilon\left|W_{m}\right|_{2,-(1+\mu), \Omega}^{2}+c(\varepsilon)\left|v_{m-1}\right|_{\infty, \Omega}^{2}\left|W_{m}\right|_{2,-\mu, \Omega}^{2} .
$$

The fifth term has the same bound. The sixth term on the 1.h.s. of (7.42) equals

$$
-\nu \int_{\Omega} \operatorname{div}\left(\nabla W_{m} W_{m} r^{-2 \mu}\right) d x+\nu \int_{\Omega}\left|\nabla W_{m}\right|^{2} r^{-2 \mu} d x-2 \mu \nu \int_{\Omega} \nabla W_{m} W_{m} r^{-2 \mu-1} \nabla r d x,
$$

where the first term equals

$$
-\nu \int_{S} \bar{n} \cdot \nabla W_{m} W_{m} r^{-2 \mu} d S \equiv I_{1},
$$

where

$$
\nu \bar{n} \cdot \nabla W_{m}+\gamma W_{m}=\nu \frac{a_{1}}{r} W_{m} \quad \text { on } S
$$

Hence

$$
I_{1}=\gamma \int_{S} W_{m}^{2} r^{-2 \mu} d S-\nu \int_{S} \frac{a_{1}}{r} W_{m}^{2} r^{-2 \mu} d S .
$$

Assuming that $\left|a_{1} / r\right| \leq c$ we estimate the second integral in $I_{1}$ by

$$
\varepsilon\left\|W_{m}\right\|_{2,1-\mu, \Omega}^{2}+c(\varepsilon)\left|W_{m}\right|_{2,1-\mu, \Omega}^{2} .
$$

The second term on the l.h.s. of (7.42) is estimated by

$$
\varepsilon\left|W_{m}\right|_{6,-\mu, \Omega}^{2}+c(\varepsilon)\left|V_{m}\right|_{3, \Omega}^{2}\left|\nabla w_{m}\right|_{2,-\mu, \Omega}^{2}
$$

The fourth term on the l.h.s. of (7.42) is bounded by

$$
\varepsilon\left|W_{m}\right|_{2,-(1+\mu), \Omega}^{2}+c(\varepsilon)\left|V_{m}\right|_{2, \Omega}^{2}\left|w_{m}\right|_{6,-\mu, \Omega}^{2} .
$$

In view of the above estimates equality (7.42) takes the form

$$
\begin{aligned}
\frac{1}{2} \frac{d}{d t}\left|W_{m}\right|_{2,-\mu, \Omega}^{2}+\nu\left|\nabla W_{m}\right|_{2,-\mu, \Omega}^{2}+\nu\left|W_{m}\right|_{2,-(1+\mu), \Omega}^{2}+\gamma\left|W_{m}\right|_{2,-\mu, S}^{2} \\
\leq c\left|v_{m-1}\right|_{\infty, \Omega}^{2}\left|W_{m}\right|_{2,-\mu, \Omega}^{2}+\varepsilon|| W_{m} \|_{2,1-\mu, \Omega}^{2} \\
\quad+c(1 / \varepsilon)\left|W_{m}\right|_{2,1-\mu, \Omega}^{2}+c\left|V_{m}\right|_{3, \Omega}^{2}\left|\nabla w_{m}\right|_{2,-\mu, \Omega}^{2}+c\left|V_{m}\right|_{2, \Omega}\left|w_{m}\right|_{6,-\mu, \Omega}^{2} \\
\quad+c\left|Q_{m}\right|_{2,-\mu, \Omega}^{2}+c\left|H_{m}\right|_{2,-(1+\mu), \Omega}^{2} .
\end{aligned}
$$

Using the estimate

$$
\left|v_{m-1}\right|_{\infty, \Omega} \leq c\left\|v_{m-1}\right\|_{3,1-\mu, \Omega}
$$

we integrate (7.43) with respect to time to get 


$$
\begin{aligned}
\frac{1}{2}\left|W_{m}\right|_{2,-\mu, \Omega}^{2}+\nu \mid & \left.\nabla W_{m}\right|_{2,-\mu, \Omega^{t}} ^{2}+\nu\left|W_{m}\right|_{2,-(1+\mu), \Omega^{t}}^{2}+\gamma\left|W_{m}\right|_{2,-\mu, S^{t}}^{2} \\
\leq & \varphi(A)\left[\int_{0}^{t}\left|V_{m}\right|_{3, \Omega}^{2}\left|\nabla w_{m}\right|_{2,-\mu, \Omega}^{2} d t^{\prime}+\int_{0}^{t}\left|V_{m}\right|_{3, \Omega}^{2}\left|w_{m}\right|_{6,-\mu, \Omega}^{2} d t^{\prime}\right. \\
& +\varepsilon \int_{0}^{t}\left\|W_{m}\right\|_{2,1-\mu, \Omega}^{2} d t^{\prime}+c(1 / \varepsilon)\left|W_{m}\right|_{2,1-\mu, \Omega^{t}}^{2}+c\left|Q_{m}\right|_{2,-\mu, \Omega^{t}}^{2} \\
& \left.+c\left|H_{m}\right|_{2,-(1+\mu), \Omega^{t}}^{2}\right],
\end{aligned}
$$

where we used the fact that

$$
\int_{0}^{t}\left\|v_{m-1}\right\|_{3,1-\mu, \Omega}^{2} d t^{\prime} \leq \varphi(A) .
$$

In view of the estimates

$$
\begin{gathered}
\sup _{t}\left|V_{m}\right|_{3, \Omega}^{2} \leq \sup _{t}\left\|V_{m}\right\|_{1,1-\mu, \Omega}^{2} \leq c t\left\|V_{m, t}\right\|_{1,1-\mu, \Omega}^{2} \leq c t\left\|V_{m}\right\|_{A_{1-\mu}\left(\Omega^{t}\right)}^{2}, \\
\left|\nabla w_{m}\right|_{2,-\mu, \Omega} \leq c\left\|w_{m}\right\|_{2,1-\mu, \Omega}, \quad\left|w_{m}\right|_{6,-\mu, \Omega} \leq c\left\|w_{m}\right\|_{2,1-\mu, \Omega},
\end{gathered}
$$

the first two terms on the r.h.s. of (7.44) are estimated by

$$
c t\left\|w_{m}\right\|_{2,1-\mu, \Omega^{t}}\left\|V_{m}\right\|_{A_{1-\mu}\left(\Omega^{t}\right)}^{2} \leq c \varphi(A) t \bar{X}^{2}\left\|V_{m}\right\|_{A_{1-\mu}\left(\Omega^{t}\right)}^{2} .
$$

The fourth term on the r.h.s. of (7.44) is estimated by

$$
c(\varepsilon) t\left|W_{m, t}\right|_{2,1-\mu, \Omega^{t}}^{2}
$$

so the sum of the third and fourth terms is bounded by

$$
c t^{a}\left\|W_{m}\right\|_{2,1-\mu, \Omega^{t}}^{2},
$$

for some $a>0$. In view of the above considerations inequality (7.44) implies

$$
\begin{aligned}
\frac{1}{2}\left|W_{m}\right|_{2,-\mu, \Omega}^{2}+\nu \mid \nabla & \left.W_{m}\right|_{2,-\mu, \Omega^{t}} ^{2}+\nu\left|W_{m}\right|_{2,-(1+\mu), \Omega^{t}}^{2}+\gamma\left|W_{m}\right|_{2,-\mu, S^{t}}^{2} \\
\leq & \varphi(A)\left[t \bar{X}^{2}\left\|V_{m}\right\|_{A_{1-\mu}\left(\Omega^{t}\right)}^{2}\right. \\
& \left.\quad t^{a}\left\|W_{m}\right\|_{2,1-\mu, \Omega^{t}}^{2}+\left|Q_{m}\right|_{2,-\mu, \Omega^{t}}^{2}+\left|H_{m}\right|_{2,-(1+\mu), \Omega^{t}}^{2}\right]
\end{aligned}
$$

Using (7.45) in (7.41) and assuming that $t$ is sufficiently small we obtain (7.39). This ends the proof.

Now we shall obtain estimates for $K_{m+1}$.

LEMMA 7.4. Let the assumptions of Lemmas 6.1 and 6.2 hold. Then

$$
\begin{aligned}
\left\|K_{m+1}\right\|_{2,2,1-\mu, \Omega^{t}} \leq & \left(\varphi(A) t^{a}+c \bar{X}+c \delta_{0}\right)\left\|V_{m}\right\|_{A_{1-\mu}\left(\Omega^{t}\right)} \\
& +\left(c \bar{X}+\varphi(A) t^{a}\right) \sup _{t}\left|K_{m+1} / r\right|_{2, \Omega}+\varphi(B)\left\|H_{m}\right\|_{2,-\mu, \Omega^{t}} \\
& +\varphi(A) \sup _{t}\left(\left|W_{m, x}\right|_{2, \Omega}+\left|W_{m}\right|_{2,-\mu, \Omega}\right)
\end{aligned}
$$

for some $a>0$, where $\delta_{0}=\|k-\gamma /(2 \nu)\|_{3 / 2, S}$. 
Proof. For solutions of problem (7.5) we have

$$
\begin{aligned}
\left\|K_{m+1}\right\|_{2,2,1} & \mu, \Omega^{t} \leq c\left[\left|v_{m} \cdot \nabla K_{m+1}\right|_{2,1-\mu, \Omega^{t}}+\left|v_{m} \cdot \nabla \chi_{m}\right|_{2,1-\mu, \Omega^{t}}+\left|\frac{\chi_{m}}{r} H_{m \varphi}\right|_{2,1-\mu, \Omega^{t}}+\left|\frac{v_{m r}}{r} K_{m+1}\right|_{2,1-\mu, \Omega^{t}}\right. \\
& +\left|\frac{h_{m \varphi}}{r} K_{m+1}\right|_{2,1-\mu, \Omega^{t}}+\left|K_{m+1}\right|_{2,-(1+\mu), \Omega^{t}}+\left|\frac{\chi_{m}}{r} V_{m r}\right|_{2,1-\mu, \Omega^{t}}+\left.\left|\frac{1}{r}\left(w_{m, z} H_{m r}-w_{m, r} H_{m z}\right)\right|_{2,1-\mu, \Omega^{t}} H_{m z}\right|_{2,1-\mu, \Omega^{t}} \\
& +\left|\frac{1}{r}\left(W_{m, z} h_{m-1 r}-W_{m, r} h_{m-1}\right)\right|_{2,1-\mu, \Omega^{t}}+\left|\frac{w_{m}}{r} V_{m \varphi, z}\right|_{2,1-\mu, \Omega^{t}} \\
& +\left|\frac{1}{r^{2}} W_{m} h_{m-1 z}\right|_{2,1-\mu, \Omega^{t}}+\left.\frac{1}{r^{2}}\left(\frac{1}{r} H_{m z, \varphi}-H_{m \varphi, z}\right)\right|_{2,1-\mu, \Omega^{t}} \\
& +\left|\frac{1}{r} v_{m-1 \varphi, z} W_{m}\right|_{2,1-\mu, \Omega^{t}} \\
& +\left\|(k-\gamma /(2 \nu)) V_{m} \cdot \bar{\tau}_{2}\right\|_{3 / 2,2,1-\mu, S^{t}} .
\end{aligned}
$$

Now we shall estimate the particular terms on the r.h.s. of (7.47). The first term is estimated by

$$
\begin{aligned}
\left(\int_{0}^{t}\left|v_{m} \cdot \nabla K_{m+1}\right|_{2,1-\mu, \Omega}^{2} d t^{\prime}\right)^{1 / 2} \leq \sup _{t \leq T}\left|v_{m}\right|_{4, \Omega}\left(\int_{0}^{t}\left|\nabla K_{m+1}\right|_{4,1-\mu, \Omega}^{2} d t^{\prime}\right)^{1 / 2} \\
\quad \leq \sup _{t \leq T}\left\|v_{m}\right\|_{1, r, 1-\mu, \Omega}\left(\int_{0}^{t}\left(\varepsilon\left|K_{m+1, x x}\right|_{2,1-\mu, \Omega}^{2}+c(1 / \varepsilon)\left|K_{m+1}\right|_{2,1-\mu, \Omega}^{2}\right) d t^{\prime}\right)^{1 / 2} \equiv I_{1},
\end{aligned}
$$

whenever $r \geq 12 / 5$, so

$$
I_{1} \leq c\left\|v_{m}\right\|_{A_{1-\mu}\left(\Omega^{T}\right)}\left(\int_{0}^{t}\left(\varepsilon\left|K_{m+1, x x}\right|_{2,1-\mu, \Omega}^{2}+c(1 / \varepsilon) t\left|K_{m+1, t}\right|_{2,1-\mu, \Omega^{t}}\right) d t\right)^{1 / 2} \equiv I_{2},
$$

where we needed that $r<6$. Hence,

$$
I_{2} \leq c\left\|v_{m}\right\|_{A_{1-\mu}\left(\Omega^{T}\right)} t^{a}\left\|K_{m+1}\right\|_{2,2,1-\mu, \Omega^{t}} \leq \varphi(A) t^{a}\left\|K_{m+1}\right\|_{2,2,1-\mu, \Omega^{t}}
$$

for some $a>0$, where to estimate the first factor we used estimates from Lemmas 6.1 and 6.2 on the interval $[0, T]$ but we examine convergence on interval $[0, t]$, where $t$ is in general very small. This interpretation implies that $c$ and $\varphi$ do not depend on $t$.

We treat the second integral on the r.h.s. of (7.47) as follows:

$$
\left(\int_{0}^{t}\left|V_{m} \cdot \nabla \chi_{m}\right|_{2,1-\mu, \Omega}^{2} d t^{\prime}\right)^{1 / 2} \leq\left(\int_{0}^{t}\left|V_{m}\right|_{6^{\prime}, 1-\mu, \Omega}^{2}\left|\nabla \chi_{m}\right|_{3^{\prime}, \Omega}^{2} d t^{\prime}\right)^{1 / 2}
$$


where $6^{\prime}<6$ but is very close to $6,6^{\prime}=2 \lambda_{1}, 3^{\prime}=2 \lambda_{2}, 1 / \lambda_{1}+1 / \lambda_{2}=1$,

$$
\begin{aligned}
& \leq \sup _{t}\left|V_{m}\right|_{6^{\prime}, 1-\mu, \Omega}\left(\int_{0}^{t}\left|\nabla \chi_{m}\left(t^{\prime}\right)\right|_{3^{\prime}, \Omega}^{2} d t^{\prime}\right)^{1 / 2} \\
& \leq \sup _{t}\left\|V_{m}\right\|_{1,1-\mu, \Omega}\left\|\chi_{m}\right\|_{2,1-\mu, \Omega^{t}} \leq \varphi(A) t^{1 / 2}\left(\int_{0}^{t}\left\|V_{m, t^{\prime}}\left(t^{\prime}\right)\right\|_{1,1-\mu, \Omega}^{2} d t^{\prime}\right)^{1 / 2} \\
& \leq \varphi(A) t^{1 / 2}\left\|V_{m}\right\|_{A_{1-\mu}\left(\Omega^{t}\right) .}
\end{aligned}
$$

We estimate the third term on the r.h.s. of (7.47) by

$$
\begin{aligned}
\left(\int_{0}^{t}\left|h_{m \varphi} \frac{K_{m+1}}{r}\right|_{2,1-\mu, \Omega}^{2} d t^{\prime}\right)^{1 / 2} & \leq \sup _{t}\left|\frac{K_{m+1}}{r}\right|_{2, \Omega}^{2}\left(\int_{0}^{t}\left|h_{m}\left(t^{\prime}\right)\right|_{\infty, 1-\mu, \Omega}^{2} d t^{\prime}\right)^{1 / 2} \\
& \leq c\left\|h_{m}\right\|_{2,1-\mu, \Omega^{t}} \sup _{t}\left|\frac{K_{m+1}}{r}\right|_{2, \Omega} \leq c \bar{X} \sup _{t}\left|\frac{K_{m+1}}{r}\right|_{2, \Omega},
\end{aligned}
$$

the fourth term by

$$
\begin{aligned}
\left(\int_{0}^{t}\left|\frac{\chi_{m}}{r} H_{m \varphi}\right|_{2,1-\mu, \Omega}^{2} d t^{\prime}\right)^{1 / 2} & \leq \sup _{t}\left|\frac{\chi_{m}}{r}\right|_{2, \Omega}\left(\int_{0}^{t}\left|H_{m}\left(t^{\prime}\right)\right|_{\infty, 1-\mu, \Omega}^{2} d t^{\prime}\right)^{1 / 2} \\
& \leq \varphi(B)\left\|H_{m}\right\|_{2,1-\mu, \Omega^{t}},
\end{aligned}
$$

the fifth term by

$$
\begin{aligned}
\left(\int_{0}^{t}\left|v_{m r} \frac{K_{m+1}}{r}\right|_{2,1-\mu, \Omega}^{2} d t^{\prime}\right)^{1 / 2} \leq \sup _{t}\left|\frac{K_{m+1}}{r}\right|_{2, \Omega}\left(\int_{0}^{t}\left|v_{m}\left(t^{\prime}\right)\right|_{\infty, 1-\mu, \Omega}^{2} d t^{\prime}\right)^{1 / 2} \\
\quad \leq \sup _{t}\left|\frac{K_{m+1}}{r}\right|_{2, \Omega}\left(\int_{0}^{t}\left\|\left.\left|v_{m}\left(t^{\prime}\right) \|_{2,2,1-\mu, \Omega}^{2 \theta}\right| v_{m}\left(t^{\prime}\right)\right|_{2,1-\mu, \Omega} ^{2(1-\theta)} d t^{\prime}\right)^{1 / 2} \quad\left(\theta=\frac{3}{4}\right)\right. \\
\leq \sup _{t}\left|\frac{K_{m+1}}{r}\right|_{2, \Omega} \sup _{t}\left|v_{m}\right|_{2,1-\mu, \Omega}^{1-\theta}\left(\int_{0}^{t}\left\|v_{m}\left(t^{\prime}\right)\right\|_{2,2,1-\mu, \Omega}^{2 \theta} d t^{\prime}\right)^{1 / 2} \\
\leq c t^{1-\theta} \sup _{t}\left|\frac{K_{m+1}}{r}\right|_{2, \Omega} \sup _{t}\left|v_{m}\right|_{2,1-\mu, \Omega}^{1-\theta}\left\|v_{m}\right\|_{2,2,1-\mu, \Omega^{t}}^{\theta} \\
\leq \varphi(A) t^{1-\theta} \sup _{t}\left|\frac{K_{m+1}}{r}\right|_{2, \Omega},
\end{aligned}
$$

the sixth term by

$$
\begin{aligned}
\left(\int_{0}^{t}\left|\frac{\chi_{m}}{r} V_{m r}\right|_{2,1-\mu, \Omega}^{2} d t^{\prime}\right)^{1 / 2} \leq \sup _{t}\left|\frac{\chi_{m}}{r}\right|_{2, \Omega}\left(\int_{0}^{t}\left|V_{m}\left(t^{\prime}\right)\right|_{\infty, 1-\mu, \Omega}^{2} d t^{\prime}\right)^{1 / 2} \\
\quad \leq \sup _{t}\left|\frac{\chi_{m}}{r}\right|_{2, \Omega}\left[\int_{0}^{t}\left(\varepsilon\left\|V_{m}\left(t^{\prime}\right)\right\|_{2,2,1-\mu, \Omega}^{2}+c(1 / \varepsilon) t^{\prime}\left|V_{m, t^{\prime}}\left(t^{\prime}\right)\right|_{2,1-\mu, \Omega^{t}}^{2}\right) d t^{\prime}\right]^{1 / 2} \\
\leq \varphi(A) t^{a}\left\|V_{m}\right\|_{2,2,1-\mu, \Omega^{t}}
\end{aligned}
$$


for some $a>0$, and the eighth term by

$$
\begin{aligned}
\left(\int_{0}^{t}\left|w_{m, x} H_{m}\right|_{2,-\mu, \Omega}^{2} d t^{\prime}\right)^{1 / 2} & \leq \sup _{t}\left|w_{m, x}\right|_{2, \Omega}\left(\int_{0}^{t}\left|H_{m}\left(t^{\prime}\right)\right|_{\infty,-\mu, \Omega}^{2} d t^{\prime}\right)^{1 / 2} \\
& \leq c \bar{X}\left(\int_{0}^{t}\left\|H_{m}\left(t^{\prime}\right)\right\|_{2,-\mu, \Omega}^{2} d t^{\prime}\right)^{1 / 2} \leq c \bar{X}\left\|H_{m}\right\|_{2,-\mu, \Omega^{t}} .
\end{aligned}
$$

Similarly we estimate the ninth term by

$$
c \sup _{t}\left|W_{m, x}\right|_{2, \Omega}|| h_{m-1} \|_{2,-\mu, \Omega^{t}} \leq c \bar{X} \sup _{t}\left|W_{m, x}\right|_{2, \Omega},
$$

and the tenth term by

$$
\begin{aligned}
\left(\int_{0}^{t}\left|w_{m} H_{m z}\right|_{2,-(1+\mu), \Omega}^{2} d t^{\prime}\right)^{1 / 2} & \leq \sup _{t}\left|w_{m}\right|_{2,-1, \Omega}\left(\int_{0}^{t}\left|H_{m}\left(t^{\prime}\right)\right|_{\infty,-\mu, \Omega}^{2} d t^{\prime}\right)^{1 / 2} \\
& \leq c \sup _{t}\left|w_{m}\right|_{2,-1, \Omega}\left\|H_{m}\right\|_{2,-\mu, \Omega^{t}} \leq c \bar{X}\left\|H_{m}\right\|_{2,-\mu, \Omega^{t}} .
\end{aligned}
$$

We majorize the eleventh term on the r.h.s. of (7.47) by

$$
\begin{aligned}
\left|W_{m} h_{m-1}\right|_{2,-(1+\mu), \Omega^{t}}= & \left(\int_{0}^{t}\left|W_{m} h_{m-1}\right|_{2,-(1+\mu), \Omega}^{2} d t^{\prime}\right)^{1 / 2} \\
& \leq \sup _{t}\left|W_{m}\right|_{2,-\mu, \Omega}\left(\int_{0}^{t}\left|h_{m-1}\left(t^{\prime}\right)\right|_{\infty,-1, \Omega}^{2} d t^{\prime}\right)^{1 / 2} \\
& \leq c\left\|h_{m-1}\right\|_{2,-1, \Omega^{t}} \sup _{t}\left|W_{m}\right|_{2,-\mu, \Omega} \leq c \bar{X} \sup _{t}\left|W_{m}\right|_{2,-\mu, \Omega} .
\end{aligned}
$$

The twelfth term is estimated by

$$
\begin{aligned}
\left(\int_{0}^{t}\left|\frac{w_{m}}{r} V_{m \varphi, z}\right|_{2,1-\mu, \Omega}^{2}\right. & \left.d t^{\prime}\right)^{1 / 2} \leq \sup _{t}\left|w_{m}\right|_{2,-1, \Omega}\left(\int_{0}^{t}\left|V_{m \varphi, z}\left(t^{\prime}\right)\right|_{\infty, 1-\mu, \Omega}^{2} d t^{\prime}\right)^{1 / 2} \\
\leq & c \sup _{t}\left\|w_{m}\right\|_{1,0, \Omega}\left(\int_{0}^{t}\left\|V_{m, z}\left(t^{\prime}\right)\right\|_{2,2,1-\mu, \Omega}^{2} d t^{\prime}\right)^{1 / 2} \leq c \bar{X}\left\|V_{m}\right\|_{A_{1-\mu}\left(\Omega^{t}\right)} .
\end{aligned}
$$

The thirteenth term is bounded by

$$
\begin{aligned}
& \left(\int_{0}^{t}\left|\frac{W_{m}}{r} v_{m-1 \varphi, z}\right|_{2,1-\mu, \Omega}^{2} d t^{\prime}\right)^{1 / 2} \leq \sup _{t}\left|W_{m}\right|_{2,-\mu, \Omega}\left(\int_{0}^{t}\left|v_{m-1 \varphi, z}\left(t^{\prime}\right)\right|_{\infty, \Omega}^{2} d t^{\prime}\right)^{1 / 2} \\
& \leq c \sup _{t}\left|W_{m}\right|_{2,-\mu, \Omega}\left(\int_{0}^{t}\|\| v_{m-1, z}\left(t^{\prime}\right) \|_{2,2,1-\mu, \Omega}^{2} d t^{\prime}\right)^{1 / 2} \leq \varphi(A) \sup _{t}\left|W_{m}\right|_{2,-\mu, \Omega},
\end{aligned}
$$

where we employed $\mu>1 / 2$.

The last but one term is estimated by

$$
\left|H_{m, x}\right|_{2,-(1+\mu), \Omega^{t}} \leq\left\|H_{m}\right\|_{2,-\mu, \Omega^{t}} .
$$

Finally, the last term is bounded by

$$
c\|k-\gamma /(2 \nu)\|_{3 / 2, S}\left\|V_{m}\right\|_{2,2,1-\mu, \Omega^{t}} .
$$


Employing the above estimates in (7.47) yields

$$
\begin{aligned}
\left\|K_{m+1}\right\|_{2,2,1-\mu, \Omega^{t}} & \leq \varphi(A) t^{a}\left\|K_{m+1}\right\|_{2,2,1-\mu, \Omega^{t}} \\
& +\left(\varphi(A) t^{a}+c \bar{X}+c \delta_{0}\right)\left\|V_{m}\right\|_{A_{1-\mu}\left(\Omega^{t}\right)} \\
& +\left(c \bar{X}+\varphi(A) t^{a}\right) \sup _{t}\left|\frac{K_{m+1}}{r}\right|_{2, \Omega}+(\varphi(B)+c \bar{X})\left\|H_{m}\right\|_{2,-\mu, \Omega^{t}} \\
& +(c \bar{X}+\varphi(A))\left(\sup _{t}\left|W_{m, x}\right|_{2, \Omega}+\sup _{t}\left|W_{m}\right|_{2,-\mu, \Omega}\right) .
\end{aligned}
$$

Assuming that $t$ is sufficiently small we obtain (7.46). This ends the proof.

Next we introduce functions $\beta_{m+1}$ by

$$
\begin{aligned}
& \beta_{m+1, t}-\nu \Delta \beta_{m+1}=0, \\
& \left.\beta_{m+1}\right|_{S}=2(k-\gamma /(2 \nu)) v_{m} \cdot \bar{\tau}_{2}, \\
& \left.\beta_{m+1}\right|_{t=0}=0 .
\end{aligned}
$$

Taking the differences

$$
B_{m+1}=\beta_{m+1}-\beta_{m}
$$

we see that they satisfy

$$
\begin{aligned}
& B_{m+1, t}-\nu \Delta B_{m+1}=0, \\
& \left.B_{m+1}\right|_{S}=2(k-\gamma /(2 \nu)) V_{m} \cdot \bar{\tau}_{2}, \\
& \left.B_{m+1}\right|_{t=0}=0
\end{aligned}
$$

Finally, we introduce

$$
K_{m+1}^{\prime}=K_{m+1}-B_{m+1} .
$$

We see that $K_{m+1}^{\prime}$ is a solution to the problem

$$
\begin{aligned}
& K_{m+1, t}^{\prime}+v_{m} \cdot \nabla K_{m+1}^{\prime}+\left(v_{m r, r}+v_{m z, z}\right) K_{m+1}^{\prime}-\nu \Delta K_{m+1}^{\prime}+\nu \frac{K_{m+1}^{\prime}}{r^{2}} \\
&+V_{m} \cdot \nabla \chi_{m}+\left(V_{m r, r}+V_{m z, z}\right) \chi_{m} \\
&= \frac{2 \nu}{r^{2}}\left(-H_{m \varphi, z}+\frac{1}{r} H_{m z, \varphi}\right)-\frac{1}{r}\left(W_{m, z} h_{m r}+w_{m-1, z} H_{m r}\right. \\
&\left.-W_{m, r} h_{m z}-w_{m-1, r} H_{m z}+\frac{W_{m}}{r} h_{m z}+\frac{w_{m-1}}{r} H_{m z}\right) \\
&+\frac{2}{r} W_{m} v_{m \varphi, z}+\frac{2}{r} w_{m-1} V_{m \varphi, z}-v_{m} \cdot \nabla B_{m+1} \\
&-\left(v_{m r, r}+v_{m z, z}\right) B_{m+1}-\nu \frac{B_{m+1}}{r^{2}}, \\
&\left.K_{m+1}^{\prime}\right|_{S}=0, \\
&\left.K_{m+1}^{\prime}\right|_{t=0}=0 .
\end{aligned}
$$

Lemma 7.5. Let the assumptions of Lemmas 6.1 and 6.2 hold. Then

$$
\left|K_{m+1}\right|_{2,-1, \Omega}+\left(\int_{0}^{t}\left|\nabla \frac{K_{m+1}}{r}\right|_{2, \Omega}^{2} d t^{\prime}\right)^{1 / 2} \leq \varphi(A)\left(t^{a}+\bar{X}\right)\left\|V_{m}\right\|_{A_{1-\mu}\left(\Omega^{t}\right)}
$$




$$
\begin{aligned}
& +c\left|H_{m}\right|_{2,-3, \Omega^{t}}+c \bar{X}\left\|H_{m}\right\|_{2,-1, \Omega^{t}} \\
& +c \bar{X}\left\|W_{m}\right\|_{2,1-\mu, \Omega^{t}}+\varphi(A) t^{a} \sup _{t}\left\|W_{m}\right\|_{1,-1 / 4-\mu^{\prime}, \Omega} \\
& +\varphi(A)\left(\int_{0}^{t}\left\|B_{m+1}\right\|_{1,-\varepsilon_{1}, \Omega}^{2} d t^{\prime}\right)^{1 / 2}+c \int_{0}^{t}\left\|B_{m+1}\left(t^{\prime}\right)\right\|_{2,-\varepsilon_{1}, \Omega}^{2} d t^{\prime}
\end{aligned}
$$

for any small positive number $\varepsilon_{1}$ and any $\mu^{\prime}<1 / 4$.

Proof. To obtain the estimate we multiply $(7.52)_{1}$ by $K_{m+1}^{\prime} / r^{2}$ and integrate the result over $\Omega^{t}$. Then we get

$$
\begin{aligned}
& \quad\left|K_{m+1}^{\prime}\right|_{2,-1, \Omega}^{2}+\int_{0}^{t}\left|\nabla \frac{K_{m+1}^{\prime}}{r}\right|_{2, \Omega}^{2} d t^{\prime} \\
& \leq \quad \int_{\Omega^{t}}\left|V_{m}\right|\left|\nabla \chi_{m}\right|\left|\frac{K_{m+1}^{\prime}}{r^{2}}\right| d x d t^{\prime}+\int_{\Omega^{t}}\left|V_{m, x}\right|\left|\chi_{m}\right|\left|\frac{K_{m+1}^{\prime}}{r^{2}}\right| d x d t^{\prime} \\
& +\left|2 \nu \int_{\Omega^{t}} \frac{1}{r^{2}}\left(-H_{m \varphi, z}+\frac{1}{r} H_{m z, \varphi}\right) \frac{K_{m+1}^{\prime}}{r^{2}} d x d t^{\prime}\right|+\int_{\Omega^{t}} \frac{1}{r^{3}}\left|W_{m, x}\right|\left|h_{m}\right|\left|K_{m+1}^{\prime}\right| d x d t^{\prime} \\
& +\int_{\Omega^{t}} \frac{1}{r^{3}}\left|w_{m-1, x}\right|\left|H_{m}\right|\left|K_{m+1}^{\prime}\right| d x d t^{\prime}+\int_{\Omega^{t}} \frac{1}{r^{4}}\left|W_{m}\right|\left|h_{m}\right|\left|K_{m+1}^{\prime}\right| d x d t^{\prime} \\
& +\int_{\Omega^{t}} \frac{1}{r^{4}}\left|w_{m-1}\right|\left|H_{m}\right|\left|K_{m+1}^{\prime}\right| d x d t^{\prime}+\int_{\Omega^{t}} \frac{1}{r^{3}}\left|W_{m}\right|\left|v_{m \varphi, z}\right|\left|K_{m+1}^{\prime}\right| d x d t^{\prime} \\
& +\int_{\Omega^{t}} \frac{1}{r^{3}}\left|w_{m-1}\right|\left|V_{m \varphi, z}\right|\left|K_{m+1}^{\prime}\right| d x d t^{\prime}+\int_{\Omega^{t}}\left|v_{m} \cdot \nabla B_{m+1}\right|\left|\frac{K_{m+1}^{\prime} \mid}{r^{2}}\right| d x d t^{\prime} \\
& +\int_{\Omega^{t}}\left|v_{m, x}\right|\left|B_{m+1}\right|\left|\frac{K_{m+1}^{\prime}}{r^{2}}\right| d x d t^{\prime}+\int_{\Omega^{t}} \frac{1}{r^{4}}\left|B_{m+1}\right|\left|K_{m+1}^{\prime}\right| d x d t^{\prime} .
\end{aligned}
$$

We estimate the first term on the r.h.s. by

$$
\varepsilon \int_{0}^{t}\left|\frac{K_{m+1}^{\prime}}{r}\right|_{6, \Omega}^{2} d t^{\prime}+c(1 / \varepsilon) \int_{0}^{t}\left|V_{m} \nabla \chi_{m}\right|_{6 / 5,-1, \Omega}^{2} d t^{\prime},
$$

where the second expression is bounded by

$$
\begin{aligned}
c \int_{0}^{t}\left|V_{m}\right|_{3,-\varepsilon, \Omega}^{2}\left|\nabla \chi_{m}\right|_{2,-1+\varepsilon, \Omega}^{2} d t^{\prime} & \leq c \sup _{t}\left\|V_{m}\right\|_{1,1-\mu, \Omega}^{2} \int_{0}^{t}\left(\left|\nabla \frac{\chi_{m}}{r^{1-\varepsilon}}\right|^{2}+\left|\frac{\chi_{m}}{r^{2-\varepsilon}}\right|^{2}\right) d t^{\prime} \\
& \leq c t \int_{0}^{t}\left\|V_{m, t^{\prime}}\right\|_{1,1-\mu, \Omega}^{2} d t^{\prime} \int_{0}^{t}\left(\left|\nabla \frac{\chi_{m}}{r}\right|^{2}+\left|\frac{\chi_{m}}{r^{2-\varepsilon}}\right|^{2}\right) d t^{\prime} \\
& \leq c \varphi(A) t\left\|V_{m}\right\|_{A_{1-\mu}\left(\Omega^{t}\right)}^{2} .
\end{aligned}
$$

We bound the second term on the r.h.s. of (7.54) by 


$$
\varepsilon \int_{0}^{t}\left|\frac{K_{m+1}^{\prime}}{r}\right|_{6, \Omega}^{2} d t^{\prime}+c(1 / \varepsilon) \int_{0}^{t}\left|V_{m, x} \chi_{m}\right|_{6 / 5,-1, \Omega}^{2} d t^{\prime},
$$

where the second expression is bounded by

$$
c \sup _{t}\left|\chi_{m}\right|_{2,-1, \Omega}^{2} \int_{0}^{t}\left|\nabla V_{m}\right|_{3, \Omega}^{2} d t^{\prime}
$$

and

$$
\int_{0}^{t}\left|\nabla V_{m}\right|_{3, \Omega}^{2} d t^{\prime} \leq \int_{0}^{t}\left|V_{m, x x x}\right|_{2,1-\mu, \Omega}^{2 \theta_{1}}\left|V_{m, x}\right|_{2,1-\mu, \Omega}^{2\left(1-\theta_{1}\right)} d t^{\prime} \equiv I_{1}
$$

for any $\theta_{1} \in(0,1)$. Continuing,

$$
\begin{aligned}
I_{1} & \leq c t^{2\left(1-\theta_{1}\right)}\left(\int_{0}^{t}\left|V_{m, x t}\right|_{2,1-\mu, \Omega}^{2} d t^{\prime}\right)^{2\left(1-\theta_{1}\right)} t^{1-\theta_{1}}\left(\int_{0}^{t}\left|V_{m, x x x}\right|_{2,1-\mu, \Omega}^{2} d t^{\prime}\right)^{\theta_{1}} \\
& \leq c t^{a}\left\|V_{m}\right\|_{A_{1-\mu}\left(\Omega^{t}\right)}^{2} \quad \text { for some } a>0 .
\end{aligned}
$$

The third term on the r.h.s. of (7.54) equals

$$
\begin{aligned}
2 \nu \mid \int_{\Omega^{t}}\left(\frac{1}{r^{3}} H_{m \varphi}\left(\frac{K_{m+1}^{\prime}}{r}\right)_{, z}-\frac{1}{r^{3}} H_{m z}\right. & \left.\frac{1}{r} \partial_{\varphi}\left(\frac{K_{m+1}^{\prime}}{r}\right)\right) d x d t^{\prime} \mid \\
& \leq \varepsilon \int_{\Omega^{t}}\left|\nabla \frac{K_{m+1}^{\prime}}{r}\right|^{2} d x d t^{\prime}+c(1 / \varepsilon)\left|H_{m}\right|_{2,-3, \Omega^{t}}^{2} .
\end{aligned}
$$

The fourth term is estimated by

$$
\varepsilon \int_{0}^{t}\left|\frac{K_{m+1}^{\prime}}{r}\right|_{6, \Omega}^{2} d t^{\prime}+c(1 / \varepsilon) \int_{0}^{t}\left|W_{m, x}\right|_{2,1-\mu, \Omega}^{2}\left|h_{m}\right|_{3,-3+\mu, \Omega}^{2} d t^{\prime},
$$

where the second expression is bounded by

$$
\begin{aligned}
c \sup _{t}\left|W_{m, x}\right|_{2,1-\mu, \Omega}^{2} \int_{0}^{t}\left\|h_{m}\right\|_{2,-1, \Omega}^{2} d t^{\prime} & \leq c\left\|W_{m}\right\|_{2,1-\mu, \Omega^{t}}^{2}\left\|h_{m}\right\|_{2,-1, \Omega^{t}}^{2} \\
& \leq c \bar{X}\left\|W_{m}\right\|_{2,1-\mu, \Omega^{t}}^{2}
\end{aligned}
$$

where we used (2.8) in the form

$$
\left|h_{m}\right|_{3,-3+\mu, \Omega} \leq c\left\|h_{m}\right\|_{2,-1, \Omega} .
$$

We estimate the fifth term exactly in the same way as the fourth. Therefore it is bounded by

$$
\varepsilon \int_{0}^{t}\left|\frac{K_{m+1}^{\prime}}{r}\right|_{6, \Omega}^{2}+c(1 / \varepsilon)\left\|w_{m}\right\|_{2,1-\mu, \Omega^{t}}^{2}\left\|H_{m}\right\|_{2,-1, \Omega^{t}}^{2},
$$

where the second expression is not greater than

$$
c \bar{X}^{2}\left\|H_{m}\right\|_{2,-1, \Omega^{t}}^{2}
$$


The sixth term is estimated by

$$
\begin{aligned}
\varepsilon \int_{0}^{t}\left|\frac{K_{m+1}^{\prime}}{r}\right|_{6, \Omega}^{2} d t^{\prime}+ & c(1 / \varepsilon) \int_{0}^{t}\left|W_{m}\right|_{2,-\mu, \Omega}^{2}\left|h_{m}\right|_{3,-3+\mu, \Omega}^{2} d t^{\prime} \\
& \leq \varepsilon \int_{0}^{t}\left|\nabla \frac{K_{m+1}^{\prime}}{r}\right|_{2, \Omega}^{2} d t^{\prime}+c(1 / \varepsilon) \sup _{t}\left|W_{m, x}\right|_{2,1-\mu, \Omega}^{2} \int_{0}^{t}\left\|h_{m}\right\|_{2,-1, \Omega}^{2} d t^{\prime} \\
& \leq \varepsilon \int_{0}^{t}\left|\nabla \frac{K_{m+1}^{\prime}}{r}\right|_{2, \Omega}^{2} d t^{\prime}+c(1 / \varepsilon) \bar{X}^{2}\left\|W_{m}\right\|_{2,1-\mu, \Omega^{t}}^{2},
\end{aligned}
$$

the seventh term by

$$
\begin{array}{r}
\varepsilon \int_{0}^{t}\left|\nabla \frac{K_{m+1}^{\prime}}{r}\right|_{2, \Omega}^{2} d t^{\prime}+c(1 / \varepsilon)\left\|w_{m}\right\|_{2,1-\mu, \Omega^{t}}^{2}\left\|H_{m}\right\|_{2,-1, \Omega^{t}}^{2} \\
\quad \leq \varepsilon \int_{0}^{t}\left|\nabla \frac{K_{m+1}^{\prime}}{r}\right|_{2, \Omega}^{2} d t^{\prime}+c(1 / \varepsilon) \bar{X}^{2}\left\|H_{m}\right\|_{2,-1, \Omega^{t}}^{2},
\end{array}
$$

and the eighth term by

$$
\varepsilon \int_{0}^{t}\left|\frac{K_{m+1}^{\prime}}{r^{2-\varepsilon^{\prime}}}\right|_{2, \Omega}^{2}+c(1 / \varepsilon) \int_{0}^{t}\left|W_{m}\right|_{4,-\mu_{1}, \Omega}^{2}\left|v_{m \varphi, z}\right|_{4,-\mu_{2}-\varepsilon^{\prime}, \Omega}^{2} d t^{\prime} \equiv I_{2}
$$

whenever $\mu_{1}+\mu_{2}=1, \mu_{1}<1 / 4, \mu_{2}>3 / 4, \varepsilon^{\prime}>0$, and by the Hardy inequality the first term in $I_{2}$ is estimated by

$$
\int_{0}^{t}\left|\nabla \frac{K_{m+1}^{\prime}}{r}\right|_{2, \Omega}^{2} d t^{\prime}
$$

Using (2.8) we estimate the second term in $I_{2}$ by

$$
c \sup _{t}\left\|W_{m}\right\|_{1,1 / 4-\mu_{1}, \Omega}^{2} \int_{0}^{t}\left|v_{m \varphi, z}\right|_{4,-\mu_{2}-\varepsilon^{\prime}, \Omega}^{2} d t^{\prime} \equiv I_{3} .
$$

To get a bound for the second factor in $I_{3}$ we use problem (6.6) for step $m$. Hence in cylindrical coordinates we have

$$
v_{m \varphi, z}=-\alpha_{m 1}+\frac{1}{r} h_{m z} .
$$

Next

$$
\int_{0}^{t}\left|v_{m \varphi, z}\left(t^{\prime}\right)\right|_{4,-\mu_{2}-\varepsilon^{\prime}, \Omega}^{2} d t^{\prime} \leq \int_{0}^{t}\left|\alpha_{m 1}\left(t^{\prime}\right)\right|_{4,-\mu_{2}-\varepsilon^{\prime}, \Omega}^{2} d t^{\prime}+\int_{0}^{t}\left|\frac{1}{r} h_{m}\left(t^{\prime}\right)\right|_{4,-\mu_{2}-\varepsilon^{\prime}, \Omega}^{2} d t^{\prime} \equiv I_{4} .
$$

Using (2.8) we have

$$
I_{4} \leq c \int_{0}^{t}\left\|\alpha_{m 1}\left(t^{\prime}\right)\right\|_{1,1 / 4-\mu_{2}-\varepsilon^{\prime}, \Omega}^{2} d t^{\prime}+c \int_{0}^{t}\left\|h_{m}\left(t^{\prime}\right)\right\|_{2,1 / 4-\mu_{2}-\varepsilon^{\prime}, \Omega}^{2} d t^{\prime} \equiv I_{5}
$$

whenever $\mu_{2} \in(3 / 4,1)$. 
In view of $(4.53),(4.32),(4.35),(6.49)$ and (6.74) we have

$$
I_{5} \leq \varphi(A) \bar{X}^{2} \text {. }
$$

Summarizing

$$
I_{3} \leq \varphi(A) \bar{X}^{2} \sup _{t}\left\|W_{m}\right\|_{1,1 / 4-\mu_{1}, \Omega}^{2} .
$$

Finally, we estimate the ninth term by

$$
\varepsilon \int_{0}^{t}\left|\frac{K_{m+1}^{\prime}}{r^{2-\varepsilon^{\prime}}}\right|_{2, \Omega}^{2} d t^{\prime}+c(1 / \varepsilon) \int_{0}^{t}\left|w_{m-1}\right|_{4,-\mu_{1}, \Omega}^{2}\left|V_{m \varphi, z}\right|_{4,-\mu_{2}-\varepsilon^{\prime}, \Omega}^{2} d t^{\prime} \equiv I_{2}^{\prime},
$$

whenever $\mu_{1}+\mu_{2}=1, \mu_{1}<1 / 4, \mu_{2}>3 / 4, \varepsilon>0$.

The first term in $I_{2}^{\prime}$ is estimated by the same bound as in $I_{2}$. By (2.8) the second term in $I_{2}^{\prime}$ is bounded by

$$
c \sup _{t}\left\|w_{m}\right\|_{1,1 / 4-\mu_{1}, \Omega}^{2} \int_{0}^{t}\left|V_{m \varphi, z}\left(t^{\prime}\right)\right|_{4,-\mu_{2}-\varepsilon^{\prime}, \Omega}^{2} d t^{\prime} \equiv I_{3}^{\prime} .
$$

By (6.67) the first factor in $I_{3}^{\prime}$ is bounded by $\varphi(A) \bar{X}^{2}$. To estimate the second factor in $I_{3}^{\prime}$ we use (7.7) for step $m$. Hence in cylindrical coordinates we have the relation

$$
V_{m \varphi, z}=-A_{m 1}+\frac{1}{r} H_{m z} .
$$

In view of (7.56) and repeating the considerations for $I_{4}$ we obtain

$$
\begin{aligned}
\int_{0}^{t}\left|V_{m \varphi, z}\left(t^{\prime}\right)\right|_{4,-\mu_{2}-\varepsilon^{\prime}, \Omega}^{2} d t^{\prime} & \leq c \int_{0}^{t}\left\|A_{m 1}\left(t^{\prime}\right)\right\|_{1,1 / 4-\mu_{2}-\varepsilon^{\prime}, \Omega}^{2} d t^{\prime}+c \int_{0}^{t}\left\|H_{m}\left(t^{\prime}\right)\right\|_{2,1 / 4-\mu_{2}-\varepsilon^{\prime}, \Omega}^{2} d t^{\prime} \\
& \leq c\left\|A_{m 1}\right\|_{1,-\delta, \Omega^{t}}^{2}+c\left\|H_{m}\right\|_{2,-\delta, \Omega^{t}}^{2}
\end{aligned}
$$

whenever $\delta \in(1 / 2,1)$.

The terms with $B_{m+1}$ are estimated by

$\varepsilon \int_{0}^{t}\left|\frac{K_{m+1}^{\prime}}{r^{2-\varepsilon_{1}}}\right|_{2, \Omega}^{2}+\int_{0}^{t} \int_{\Omega}\left[r^{-2 \varepsilon_{1}}\left(\left|v_{m}\right|^{2}\left|\nabla B_{m+1}\right|^{2}+\left|v_{m, x}\right|^{2}\left|B_{m+1}\right|^{2}\right)+r^{-2\left(2+\varepsilon_{1}\right)}\left|B_{m+1}\right|^{2}\right] d x d t^{\prime}$

for any $\varepsilon_{1}>0$ close to 0 and we bound the second integral by

$$
\begin{aligned}
& c \sup _{t}\left|v_{m}\right|_{\infty, \Omega}^{2} \int_{0}^{t}\left|\nabla B_{m+1}\right|_{2,-\varepsilon_{1}, \Omega}^{2} d t^{\prime}+c \sup _{t}\left|v_{m, x}\right|_{3, \Omega}^{2} \int_{0}^{t}\left|B_{m+1}\right|_{6,-\varepsilon_{1}, \Omega}^{2} d t^{\prime} \\
& \quad+c \int_{0}^{t}\left|B_{m+1}\right|_{2,-\left(2+\varepsilon_{1}\right), \Omega}^{2} d t^{\prime} \\
& \leq c\left(\left\|v_{m}\right\|_{A_{1-\mu}\left(\Omega^{T}\right)}+\|v(0)\|_{2, \Omega}\right)^{2} \int_{0}^{t}\left\|B_{m+1}\right\|_{1,-\varepsilon_{1}, \Omega}^{2} d t^{\prime}+c \int_{0}^{t}\left|B_{m+1}\right|_{2,-\left(2+\varepsilon_{1}\right), \Omega}^{2} d t^{\prime} \\
& \leq \varphi(A) \int_{0}^{t}\left\|B_{m+1}\right\|_{1,-\varepsilon_{1}, \Omega}^{2} d t^{\prime}+c \int_{0}^{t}\left\|B_{m+1}\right\|_{2,-\varepsilon_{1}, \Omega}^{2} d t^{\prime} .
\end{aligned}
$$


Employing the above estimates in (7.54) we obtain

$$
\begin{aligned}
\left|K_{m+1}^{\prime}\right|_{2,-1, \Omega}^{2}+\int_{0}^{t}\left|\nabla \frac{K_{m+1}^{\prime}}{r}\right|_{2, \Omega}^{2} d t^{\prime} \leq \varphi(A)\left(t^{a}+\bar{X}^{2}\right)\left\|V_{m}\right\|_{A_{1-\mu}\left(\Omega^{t}\right)}^{2} \\
\quad+c\left|H_{m}\right|_{2,-3, \Omega^{t}}^{2}+c \bar{X}^{2}\left\|W_{m}\right\|_{2,1-\mu, \Omega^{t}}^{2}+\varphi(A) t_{t}^{a} \sup _{t}\left|W_{m}\right|_{2,-1, \Omega}^{2} \\
\quad+c \bar{X}^{2}\left\|H_{m}\right\|_{2,-1, \Omega^{t}}^{2}+\varphi(A) \int_{0}^{t}\left\|B_{m+1}\right\|_{1,-\varepsilon_{1}, \Omega}^{2} d t^{\prime}+c \int_{0}^{t}\left\|B_{m+1}\right\|_{2,-\varepsilon_{1}, \Omega}^{2} d t^{\prime}
\end{aligned}
$$

for any small $\varepsilon_{1}>0$.

Using (7.51) we obtain from (7.57) inequality (7.53). This concludes the proof.

Finally, we have

TheOREM 7.6. Let the assumption of Lemmas 6.1 and 6.2 hold. Then

$$
\left\|V_{m+1}\right\|_{A_{1-\mu}\left(\Omega^{t}\right)} \leq \varphi(A, B)\left(t^{a}+\bar{X}+\delta_{0}\right)\left\|V_{m}\right\|_{A_{1-\mu}\left(\Omega^{t}\right)},
$$

for any $\delta \geq 1$, where $\delta_{0}=\|k-\gamma /(2 \nu)\|_{V_{4,-\delta / 2}^{3 / 2}(S)}$.

Proof. From (7.8) we have

$$
\begin{aligned}
\left\|A_{m+1}^{\prime}\right\|_{2,2,1-\mu, \Omega^{t}}+\left|A_{m+1}^{\prime}\right|_{2, \Omega} & +\left|A_{1 m+1}\right|_{2,-(1+\mu), \Omega^{t}} \\
\leq & \varphi(A)\left[\bar{X} \sup _{t}\left|K_{m+1}\right|_{2,-1, \Omega}+\left\|V_{m}\right\|_{A_{1-\mu}\left(\Omega^{t}\right)}\right) \\
& \left.+\left\|W_{m}\right\|_{2,1-\mu, \Omega^{t}}+\left\|H_{m}\right\|_{2,-1, \Omega^{t}}\right] .
\end{aligned}
$$

From (7.37) we get

$$
\left\|H_{m}\right\|_{2,-\mu^{\prime}, \Omega^{t}}+\left|\nabla Q_{m}\right|_{2,-\mu^{\prime}, \Omega^{t}} \leq c t^{1 / 2} \bar{X}\left\|V_{m}\right\|_{A_{1-\mu}\left(\Omega^{t}\right)}
$$

for any $\mu^{\prime} \in(0,1]$.

Next (7.39) implies

$$
\begin{aligned}
\sup _{t}\left|W_{m}\right|_{2,-\mu, \Omega}+ & \left(\int_{0}^{t}\left\|W_{m}\left(t^{\prime}\right)\right\|_{1,-\mu, \Omega}^{2} d t^{\prime}\right)^{1 / 2}+\left\|W_{m}\right\|_{2,1-\mu, \Omega^{t}} \\
& \leq \varphi(A)\left[t^{a}\left\|V_{m}\right\|_{A_{1-\mu}\left(\Omega^{t}\right)}+\left|Q_{m}\right|_{2,-\mu, \Omega^{t}}+\left|H_{m}\right|_{2,-(1+\mu), \Omega^{t}}\right] \\
& \leq \varphi(A) t^{a}\left\|V_{m}\right\|_{A_{1-\mu}\left(\Omega^{t}\right)},
\end{aligned}
$$

where (7.60) was used to get the second inequality. From (7.46) we obtain

$$
\begin{aligned}
\left\|K_{m+1}\right\|_{2,2,1-\mu, \Omega^{t} \leq} & \left(\varphi(A) t^{a}+c \bar{X}+c \delta_{0}^{\prime}\right)\left\|V_{m}\right\|_{A_{1-\mu}\left(\Omega^{t}\right)} \\
& +\left(c \bar{X}+\varphi(A) t^{a}\right) \sup _{t}\left|K_{m+1} / r\right|_{2, \Omega}+\varphi(B)\left\|H_{m}\right\|_{2,-\mu, \Omega^{t}} \\
& +\varphi(A) \sup _{t}\left(\left|W_{m, x}\right|_{2, \Omega}+\left|W_{m}\right|_{2,-\mu, \Omega}\right) \\
\leq & \varphi(A, B)\left(t^{a}+\bar{X}+\delta_{0}^{\prime}\right)\left\|V_{m}\right\|_{A_{1-\mu}\left(\Omega^{t}\right)} \\
& +c\left(\bar{X}+\varphi(A) t^{a}\right) \sup _{t}\left|K_{m+1} / r\right|_{2, \Omega},
\end{aligned}
$$

where $\delta_{0}^{\prime}=\|k-\gamma /(2 \nu)\|_{3 / 2, S}$ and the second inequality follows from (7.60) and (7.61). 
From (7.53) we get

$$
\begin{aligned}
\left|K_{m+1}\right|_{2,-1, \Omega}+ & \left(\int_{0}^{t}\left|\nabla \frac{K_{m+1}}{r}\right|_{2, \Omega}^{2} d t^{\prime}\right)^{1 / 2} \leq c\left\|H_{m}\right\|_{2,-\mu, \Omega^{t}} \\
& +c \bar{X}\left(\left\|W_{m}\right\|_{2,1-\mu, \Omega^{t}}+\left\|A_{m 1}\right\|_{1,-\delta, \Omega^{t}}+\left\|H_{m}\right\|_{2,-\delta, \Omega^{t}}\right) \\
& +\varphi(A) t^{a} \sup _{t}\left\|W_{m}\right\|_{1,1 / 4-\mu^{\prime}, \Omega}+\varphi(A)\left(\int_{0}^{t}\left\|B_{m+1}\right\|_{1,-\varepsilon_{1}, \Omega}^{2} d t^{\prime}\right)^{1 / 2} \\
& +c\left(\int_{0}^{t}\left\|B_{m+1}\left(t^{\prime}\right)\right\|_{2,-\varepsilon_{1}, \Omega}^{2} d t^{\prime}\right)^{1 / 2}
\end{aligned}
$$

for any $\delta \in(1 / 2,1), \varepsilon_{1}>0, \mu^{\prime}<1 / 4$.

For solutions of problem (7.50) we have

$$
\left(\int_{0}^{t}\left\|B_{m+1}\right\|_{1,-\varepsilon_{1}, \Omega}^{2} d t^{\prime}\right)^{1 / 2}+\left(\int_{0}^{t}\left\|B_{m+1}\left(t^{\prime}\right)\right\|_{2,-\varepsilon_{1}, \Omega}^{2} d t^{\prime}\right)^{1 / 2} \leq c \delta_{0}\left\|V_{m}\right\|_{A_{1-\mu}\left(\Omega^{t}\right)}
$$

for any $\delta \geq 1$, where $\delta_{0}=\|k-\gamma /(2 \nu)\|_{V_{4,-\delta / 2}^{3 / 2}(S)}$ and $\varepsilon_{1}=\delta / 2$. For more details see the proof of Lemma 4.1 .

Employing (7.64), (7.60) and (7.61) in (7.63) yields

$$
\left|K_{m+1}\right|_{2,-1, \Omega}+\left(\int_{0}^{t}\left|\nabla \frac{K_{m+1}}{r}\right|_{2, \Omega}^{2} d t^{\prime}\right)^{1 / 2} \leq \varphi(A, B)\left(\bar{X}+t^{a}+\delta_{0}\right)\left\|V_{m}\right\|_{A_{1-\mu}\left(\Omega^{t}\right)},
$$

where to estimate the norm with $A_{m 1}$ we use problem (7.7) for step $m$.

Using (7.65) in (7.62) and (7.59) we obtain

$$
\left\|A_{m+1}\right\|_{2,2,1-\mu, \Omega^{t}} \leq \varphi(A, B)\left(\bar{X}+t^{a}+\delta_{0}\right)\left\|V_{m}\right\|_{A_{1-\mu}\left(\Omega^{t}\right)} .
$$

For solutions of problem (7.7) we have

$$
\left\|V_{m+1}\right\|_{A_{1-\mu}\left(\Omega^{t}\right)} \leq c\left\|A_{m+1}\right\|_{2,2,1-\mu, \Omega^{t}} .
$$

From (7.67) and (7.66) we obtain (7.58). This ends the proof.

From (7.58) we have convergence of the sequence constructed in Section 6 in a small interval $[0, t]$.

THEOREM 7.7. Let the assumptions of Lemmas 6.1 and 6.2 hold. Then the sequence constructed in Section 6 converges.

Proof. By Lemmas 7.1-7.6 the sequence converges in a small interval $[0, t]$. Applying Lemmas 6.1 and 6.2 we can extend the considerations in this section step by step up to $T$. This ends the proof.

\subsection{Local existence}

Proof of Theorem 1.1. Lemmas 6.1, 6.2 and Theorem 7.7 imply Theorem 1.1. 


\section{Global existence}

8.1. Idea of the proof. To prove global existence we have to prolong the local solution from Sections 6 and 7 step by step up to infinity. To make this possible we have to prove local existence for sufficiently large time $T$. Moreover, we have to show that all quantities collected in $\bar{X}, Y_{1}, Y_{2}, Y_{3}$ (see Lemma 6.2) do not increase if we pass from the interval $[k T,(k+1) T]$ to $[(k+1) T,(k+2) T], k \in \mathbb{N}$.

For this purpose we distribute all the quantities into the following four new quantities:

$$
\begin{aligned}
Q_{1}(t) & =\|h(t)\|_{1,-1, \Omega}+\|w(t)\|_{1,0, \Omega}+\left\|\alpha^{\prime}(t)\right\|_{1,2,1-\mu, \Omega}, \\
Q_{2}(t) & =\|\chi(t)\|_{1,1-\mu, \Omega}+|\chi(t)|_{2,-1, \Omega}+\|v(t)\|_{2,2,1-\mu, \Omega}, \\
P_{1}(0, t) & =|g|_{2,1, \Omega^{t}}+|g|_{2,-1, \Omega^{t}}+\left|f_{\varphi}\right|_{2,-\mu, \Omega^{t}}+\left|F^{\prime}\right|_{2, \Omega^{t}}+\left|F_{1}\right|_{2,-\mu, \Omega^{t}}, \\
P_{2}(0, t) & =\left|F_{2}\right|_{2,-1, \Omega^{t}},
\end{aligned}
$$

where $Q_{1}, P_{1}$ are sufficiently small and the magnitudes of $P_{2}, Q_{2}$ are not restricted.

To prove global existence we have to show that $Q_{1}(t), Q_{2}(t), P_{1}(0, t), P_{2}(0, t)$ can be estimated by some quantities $Q_{1}^{*}, Q_{2}^{*}, P_{1}^{*}, P_{2}^{*}$ for any $t \in \mathbb{R}_{+}$. For this purpose we have to show that

$$
\|v\|_{A_{1-\mu}(\Omega \times(k T,(k+1) T))} \leq A, \quad \forall k \in \mathbb{N} .
$$

To simplify notation we introduce

$$
A\left(t_{1}, t_{2}\right)=\|v\|_{A_{1-\mu}\left(\Omega \times\left(t_{1}, t_{2}\right)\right)} .
$$

To show

$$
Q_{i}(t) \leq Q_{i}^{*}, \quad P_{i}(0, t) \leq P_{i}^{*}, \quad i=1,2, t \in \mathbb{R}_{+},
$$

we have to obtain some decay estimates.

\subsection{Decay estimates. First we assume}

$$
\gamma(t) \equiv|g(t)|_{2,-1, \Omega}+\left|g_{t}(t)\right|_{2, \Omega} \leq \gamma(0) e^{-\nu_{0} t}, \quad \nu_{0}>0 .
$$

Repeating the proof of Lemma 7.4.1 from [zaj 5] we have

Lemma 8.1. Assume that $v \in A_{1-\mu}\left(\Omega^{t}\right)$ and $h$ is a solution of (1.4). Assume that $0<$ $t_{1}<t<T$. Assume (8.5). Then

$$
\|h(t)\|_{1,-1, \Omega} \leq \varphi(A(0, t)) e^{-\nu_{0} t_{1}}\left(\|h(0)\|_{1,-1, \Omega}+\gamma(0)\right),
$$

where $\varphi$ is an increasing positive function and $t_{1}$ which is close to $t$ can be chosen large.

Let us introduce the quantity

$$
Z_{1}(t) \equiv\|w(t)\|_{1,0, \Omega}
$$


Now we prove a result similar to Lemma 7.4.2 from [zaj 5].

Lemma 8.2. Assume that $v \in A_{1-\mu}\left(\Omega^{t}\right)$ and (8.5) holds. Assume the growth condition (8.16). Assume the geometry of the boundary such that (8.19) holds. Assume $h_{, t}(0) \in$ $L_{2}(\Omega), h(0) \in H_{-1}^{1}(\Omega), w(0) \in H_{0}^{1}(\Omega)$. Then

$$
\begin{aligned}
Z_{1}(t) \leq & \varphi(A(0, t))\left[|w(0)|_{2,1, \Omega}+\left|h_{, t}(0)\right|_{2, \Omega}+\|h(0)\|_{1,-1, \Omega}\right. \\
& \left.+\gamma(0)+\gamma_{1}(0)+e^{-t} Z_{1}(0)+e^{-\nu t}|w(0)|_{2, \Omega}\right] .
\end{aligned}
$$

Proof. From (6.3.41) in [zaj 5] we have

$$
\begin{aligned}
\left|h_{, t}(t)\right|_{2, \Omega}^{2}+\|h(t)\|_{1, \Omega}^{2} \leq e^{-\nu_{0} t+c\|v\|_{A_{1-\mu}\left(\Omega^{t}\right)}}\left[\left|h_{, t}(0)\right|_{2, \Omega}^{2}\right. & \\
+ & \left.\|h(0)\|_{1, \Omega}^{2}+c \int_{0}^{t}\left(\left|g\left(t^{\prime}\right)\right|_{2, \Omega}^{2}+\left|g, t\left(t^{\prime}\right)\right|_{2, \Omega}^{2}\right) e^{\nu_{0} t^{\prime}-c\|v\|_{A_{1-\mu}\left(\Omega t^{\prime}\right)}} d t^{\prime}\right] \\
\leq & \varphi(A(0, t)) e^{-\nu_{0} t}\left[\left|h_{, t}(0)\right|_{2, \Omega}^{2}+\|h(0)\|_{1, \Omega}^{2}+\gamma^{2}(0)\right],
\end{aligned}
$$

where (8.5) was used in the second inequality and the constants $c$ do not depend on $t$.

From (7.4.24) in [zaj 5] we obtain

$$
|q(t)|_{2, \Omega}^{2} \leq \varphi(A(0, t)) e^{-\nu_{0} t}\left[\left|h_{, t}(0)\right|_{2, \Omega}^{2}+\|h(0)\|_{1, \Omega}^{2}+\gamma^{2}(0)\right] .
$$

Moreover (7.4.25) in [zaj 5] gives

$$
\|h\|_{2,-1, \Omega^{t}}+\left(\int_{0}^{t}\left\|q\left(t^{\prime}\right)\right\|_{1,-1, \Omega}^{2} d t^{\prime}\right)^{1 / 2} \leq \varphi(A(0, t))\left(\gamma(0)+\|h(0)\|_{1,-1, \Omega}\right) .
$$

From (5.13),

$$
\begin{aligned}
Z_{1}(t) \leq & \varphi(A(0, t))\left[\sup _{t^{\prime} \leq t}\left|w\left(t^{\prime}\right)\right|_{2,1, \Omega}+\left(e^{-t} \int_{0}^{t}\left|w\left(t^{\prime}\right)\right|_{2, \Omega}^{2} e^{t^{\prime}} d t^{\prime}\right)^{1 / 2}\right. \\
& \left.+\left(\int_{0}^{t}\left(\left|q\left(t^{\prime}\right)\right|_{2,-1, \Omega}^{2}+\left|h\left(t^{\prime}\right)\right|_{2,-2, \Omega}^{2}+\left|f_{\varphi}\left(t^{\prime}\right)\right|_{2, \Omega}^{2}\right) d t^{\prime}\right)^{1 / 2}+e^{-t} Z_{1}(0)\right] .
\end{aligned}
$$

In view of (8.11) we simplify (8.12) to

$$
\begin{aligned}
Z_{1}(t) \leq & \varphi(A(0, t))\left[\sup _{t^{\prime} \leq t}\left|w\left(t^{\prime}\right)\right|_{2,1, \Omega}+\left(e^{-t} \int_{0}^{t}\left|w\left(t^{\prime}\right)\right|_{2, \Omega}^{2} e^{t^{\prime}} d t^{\prime}\right)^{1 / 2}\right. \\
& \left.+\gamma(0)+\|h(0)\|_{1,-1, \Omega}+\left|f_{\varphi}\right|_{2, \Omega^{t}}+e^{-t} Z_{1}(0)\right] .
\end{aligned}
$$

To examine the first term on the r.h.s. we use Lemma 5.6. Hence we have

$$
|w(t)|_{2,1, \Omega} \leq|w(0)|_{2,1, \Omega}+\int_{0}^{t}\left(\left|q\left(t^{\prime}\right)\right|_{2, \Omega}+\left|h\left(t^{\prime}\right)\right|_{2,-1, \Omega}+\left|f_{\varphi}\left(t^{\prime}\right)\right|_{2, \Omega}\right) d t^{\prime} .
$$

In view of (8.6) and (8.10) we obtain

$$
\begin{aligned}
|w(t)|_{2,1, \Omega} \leq & |w(0)|_{2,1, \Omega}+\varphi(A(0, t))\left[\left|h_{, t}(0)\right|_{2, \Omega}+\|h(0)\|_{1,-1, \Omega}+\gamma(0)\right] \\
& +\int_{0}^{t}\left|f_{\varphi}\left(t^{\prime}\right)\right|_{2, \Omega} d t^{\prime}
\end{aligned}
$$


Assuming the growth condition

$$
\gamma_{1}(t) \equiv\left|f_{\varphi}(t)\right|_{2, \Omega} \leq \gamma_{1}(0) e^{-\nu_{1} t}, \quad \nu_{1}>0,
$$

and inserting it in (8.15) implies

$$
\begin{aligned}
|w(t)|_{2,1, \Omega} \leq & |w(0)|_{2,1, \Omega}+\varphi(A(0, t))\left[\left|h_{, t}(0)\right|_{2, \Omega}+\|h(0)\|_{1,-1, \Omega}+\gamma(0)\right] \\
& +c \gamma_{1}(0)
\end{aligned}
$$

In view of (8.17) estimate (8.13) takes the form

$$
\begin{aligned}
Z_{1}(t) \leq & \varphi(A(0, t))\left[|w(0)|_{2,1, \Omega}+\left|h_{, t}(0)\right|_{2, \Omega}+\|h(0)\|_{1,-1, \Omega}\right. \\
& \left.+\gamma(0)+\gamma_{1}(0)+\left(e^{-t} \int_{0}^{t}\left|w\left(t^{\prime}\right)\right|_{2, \Omega}^{2} e^{t^{\prime}} d t^{\prime}\right)^{1 / 2}+e^{-t} Z_{1}(0)\right] .
\end{aligned}
$$

Finally, we have to estimate the integral on the r.h.s. of (8.18). For this purpose we use the proof of Lemma 5.3. We exploit formula (5.8). Assuming that

$$
\left|a_{1}\right| \leq c r^{3} \quad \text { in a neighbourhood of } r=0,
$$

we can write (5.8) in the form

$$
\frac{d}{d t}|w|_{2, \Omega}^{2}+\nu|w|_{2, \Omega}^{2} \leq c|w|_{2,1, \Omega}^{2}+c\left|v_{r}\right|_{\infty, \Omega}^{2}|w|_{2, \Omega}^{2}+c\left(|q|_{2, \Omega}^{2}+|h|_{2,-1, \Omega}^{2}+\left|f_{\varphi}\right|_{2, \Omega}^{2}\right)
$$

Since $\int_{0}^{t}\left|v_{r}\right|_{\infty, \Omega}^{2} d t^{\prime} \leq c\|v\|_{A_{1-\mu}\left(\Omega^{t}\right)}^{2}$ for $\mu \in(1 / 2,1)$ we have

$$
\begin{aligned}
& \frac{d}{d t}\left(|w|_{2, \Omega}^{2} e^{\nu t-c\|v\|_{A_{1-\mu}\left(\Omega^{t}\right)}^{2}}\right) \\
& \quad \leq c\left[|w|_{2,1, \Omega}^{2}+|q|_{2, \Omega}^{2}+|h|_{2,-1, \Omega}^{2}+\left|f_{\varphi}\right|_{2, \Omega}^{2}\right] e^{\nu t-c\|v\|_{A_{1-\mu}\left(\Omega^{t}\right)}^{2}} .
\end{aligned}
$$

Integrating (8.21) with respect to time yields

$$
\begin{aligned}
|w(t)|_{2, \Omega}^{2} \leq & \varphi(A(0, t))\left[\sup _{t^{\prime} \leq t}\left|w\left(t^{\prime}\right)\right|_{2,1, \Omega}^{2}\right. \\
& \left.+\int_{0}^{t}\left(\left|q\left(t^{\prime}\right)\right|_{2, \Omega}^{2}+\left|h\left(t^{\prime}\right)\right|_{2,-1, \Omega}^{2}+\left|f_{\varphi}\left(t^{\prime}\right)\right|_{2, \Omega}^{2}\right) d t^{\prime}+e^{-\nu t}|w(0)|_{2, \Omega}^{2}\right] .
\end{aligned}
$$

Using (8.11), (8.16) and (8.17) in (8.22) implies

$$
\begin{aligned}
|w(t)|_{2, \Omega} \leq & \varphi(A(0, t))\left[|w(0)|_{2,1, \Omega}+\left|h_{, t}(0)\right|_{2, \Omega}+\|h(0)\|_{1,-1, \Omega}+\gamma(0)\right. \\
& \left.+\gamma_{1}(0)+e^{-\nu t}|w(0)|_{2, \Omega}\right] .
\end{aligned}
$$

Employing (8.23) in (8.18) we have

$$
\begin{aligned}
Z_{1}(t)+|w(t)|_{2, \Omega} \leq & \varphi(A(0, t))\left[|w(0)|_{2,1, \Omega}+\left|h_{, t}(0)\right|_{2, \Omega}+\|h(0)\|_{1,-1, \Omega}\right. \\
& \left.+\gamma(0)+\gamma_{1}(0)+e^{-t} Z_{1}(0)+e^{-\nu t}|w(0)|_{2, \Omega}\right] .
\end{aligned}
$$

This estimate implies (8.8). This concludes the proof.

Now we obtain a decay estimate for $\chi$.

Lemma 8.3. Assume that $v \in A_{1-\mu}\left(\Omega^{t}\right), F_{\varphi} \in L_{2,-1}\left(\Omega^{t}\right), k-\gamma /(2 \nu) \in H^{3 / 2}(S)$ and $k-\gamma /(2 \nu) \in V_{4,-\delta / 2}^{3 / 2}(S), \delta \geq 1, w(0) \in L_{2,1}(\Omega), h(0) \in H_{-1}^{1}(\Omega), h_{, t}(0) \in L_{2}(\Omega)$, 
$\chi(0) \in L_{2,-1}(\Omega)$ and the decay estimates (8.5) and (8.16) hold. Then

$$
\begin{aligned}
|\chi(t)|_{2,-1, \Omega} \leq & \varphi(A(0, t))\left[|w(0)|_{2,1, \Omega}+\left|h_{, t}(0)\right|_{2, \Omega}+\|h(0)\|_{1,-1, \Omega}\right. \\
& \left.+\gamma(0)+\gamma_{1}(0)+e^{-t} Z_{1}(0)+e^{-\nu t}|w(0)|_{2, \Omega}\right]+c\left|F_{\varphi}\right|_{2,-1, \Omega^{t}} \\
& +c\|k-\gamma /(2 \nu)\|_{3 / 2,4,-\delta / 2, S}\|v\|_{A_{1-\mu}\left(\Omega^{t}\right)}+e^{-\nu t}|\chi(0)|_{2,-1, \Omega} .
\end{aligned}
$$

Proof. By the local existence result $\sup _{t}\left|h_{\varphi}\right|_{2,-1, \Omega}$ is sufficiently small. Then (4.4) implies

$$
\begin{aligned}
\frac{d}{d t}\left|\chi^{\prime}\right|_{2,-1, \Omega}^{2} & +\nu\left|\chi^{\prime}\right|_{2,-1, \Omega}^{2} \leq c\left(1+\|w\|_{1,0, \Omega}^{2}\right)\|h\|_{2,-1, \Omega}^{2} \\
& +\|w\|_{1,0, \Omega}^{2}\left\|v_{\varphi}\right\|_{3,2,1-\mu, \Omega}^{2}+c|w|_{2,-1, \Omega}^{2}\left|v_{\varphi, z}\right|_{4,-3 / 4-\varepsilon, \Omega}^{2}+c\left|F_{\varphi}\right|_{2,-1, \Omega}^{2} \\
& +c\left(\left|h_{\varphi}\right|_{4, \Omega}^{2}|\beta|_{4,-(1+\delta / 2), \Omega}^{2}+\left|v^{\prime}\right|_{10 / 3, \Omega}^{2}|\beta|_{5,-1, \Omega}^{2}+|\beta|_{2,-(2+\delta / 2), \Omega}^{2}\right),
\end{aligned}
$$

where $\varepsilon>0, \delta>0$. Integrating (8.26) with respect to time yields

$$
\begin{aligned}
\left|\chi^{\prime}(t)\right|_{2,-1, \Omega}^{2} \leq & c\left(1+\sup _{t}\|w\|_{1,0, \Omega}^{2}\right) \int_{0}^{t}\left\|h\left(t^{\prime}\right)\right\|_{2,-1, \Omega}^{2} d t^{\prime} \\
& +c \sup _{t}\|w\|_{1,0, \Omega}^{2}\left\|v_{\varphi}\right\|_{A_{1-\mu}\left(\Omega^{t}\right)}^{2}+c\left|F_{\varphi}\right|_{2,-1, \Omega^{t}}^{2} \\
& +c\left(\left|h_{\varphi}\right|_{4, \Omega^{t}}^{2}|\beta|_{4,-(1+\delta / 2), \Omega^{t}}^{2}+|v|_{10 / 3, \Omega^{t}}^{2}|\beta|_{5,-1, \Omega^{t}}^{2}+|\beta|_{2,-(2+\delta / 2), \Omega^{t}}^{2}\right) \\
& +e^{-\nu t}\left|\chi^{\prime}(0)\right|_{2,-1, \Omega}^{2} .
\end{aligned}
$$

Using (8.8) and (8.11) we have

$$
\begin{aligned}
\left|\chi^{\prime}(t)\right|_{2,-1, \Omega} \leq \varphi(A(0, t))\left[|w(0)|_{2,1, \Omega}+\left|h_{, t}(0)\right|_{2, \Omega}+\|h(0)\|_{1,-1, \Omega}+\gamma(0)\right. \\
\left.\quad+\gamma_{1}(0)+e^{-t} Z_{1}(0)+e^{-\nu t}|w(0)|_{2, \Omega}\right]+c\left|F_{\varphi}\right|_{2,-1, \Omega^{t}} \\
\quad+c\left(|\beta|_{4,-(1+\delta / 2), \Omega^{t}}+|\beta|_{5,-1, \Omega^{t}}+|\beta|_{2,-(2+\delta / 2), \Omega^{t}}\right)+e^{-\nu t}\left|\chi^{\prime}(0)\right|_{2,-1, \Omega},
\end{aligned}
$$

where we have used the fact that $|v|_{10 / 3, \Omega^{t}} \leq c$ in view of the energy estimate.

Finally, the terms involving $\beta$ are estimated by

$$
c\|k-\gamma /(2 \nu)\|_{3 / 2,4,-\delta / 2, S}\|v\|_{A_{1-\mu}\left(\Omega^{t}\right)},
$$

where we have applied estimate (4.7).

Hence (8.28) implies

$$
\begin{aligned}
\left|\chi^{\prime}(t)\right|_{2,-1, \Omega} \leq & \varphi(A(0, t))\left[|w(0)|_{2,1, \Omega}+\left|h_{, t}(0)\right|_{2, \Omega}+\|h(0)\|_{1,-1, \Omega}\right. \\
& \left.+\gamma(0)+\gamma_{1}(0)+e^{-t} Z_{1}(0)+e^{-\nu t}|w(0)|_{2, \Omega}\right]+c\left|F_{\varphi}\right|_{2,-1, \Omega^{t}} \\
& +c\|k-\gamma /(2 \nu)\|_{3 / 2,4,-\delta / 2, S}\|v\|_{A_{1-\mu}\left(\Omega^{t}\right)}+e^{-\nu t}\left|\chi^{\prime}(0)\right|_{2,-1, \Omega} .
\end{aligned}
$$

Using again problem (3.33) for $\beta$ we obtain from (8.29) the inequality (8.25). This concludes the proof.

Lemma 8.4. Assume that $A(k T,(k+1) T) \leq A$ for all $k \in \mathbb{N}$,

$$
Z_{2}(t)=\left|h_{, t}(t)\right|_{2, \Omega}+\|h(t)\|_{1, \Omega}+\gamma(t),
$$

$\varphi(A) e^{-\nu_{0} T}<1, \int_{0}^{\infty}\left|f_{\varphi}\left(t^{\prime}\right)\right|_{2,1, \Omega} d t^{\prime} \leq \varepsilon_{1}$ and $\varepsilon_{1}$ is sufficiently small. Then

$$
|w(k T)|_{2,1, \Omega} \leq|w(0)|_{2,1, \Omega}+\frac{\varphi(A)}{1-\varphi(A) e^{-\nu_{0} T}} Z_{2}(0)+c \int_{0}^{\infty}\left|f_{\varphi}\left(t^{\prime}\right)\right|_{2,1, \Omega} d t^{\prime}
$$

for any $k \in \mathbb{N}$. 
Proof. From (8.9) and (8.17) we have

$$
|w(k T)|_{2,1, \Omega} \leq|w(0)|_{2,1, \Omega}+\sum_{i=0}^{k-1}\left(\varphi(A) e^{-\nu_{0} T}\right)^{i} \varphi(A) Z_{2}(0)+c \int_{0}^{k T}\left|f_{\varphi}\left(t^{\prime}\right)\right|_{2,1, \Omega} d t^{\prime} .
$$

Passing with $k$ to $\infty$ and using the assumptions of the lemma we obtain (8.30). This concludes the proof.

To obtain the time behaviour of $Q_{1}(t)$ we need an estimate for $\left\|\alpha^{\prime}(t)\right\|_{1,1-\mu, \Omega}$.

Lemma 8.5. Assume that $v \in A_{1-\mu}\left(\Omega^{T}\right), h_{, t}(0) \in L_{2}(\Omega), h(0) \in H_{-1}^{1}(\Omega), w(0) \in$ $H_{0}^{1}(\Omega), g(0) \in L_{2,-1}(\Omega), g_{, t}(0) \in L_{2}(\Omega), f_{\varphi}(0) \in L_{2}(\Omega), v(0) \in L_{2}(\Omega)$. Assume the decay estimates (8.5), (8.16). Assume that there exists a local solution determined by Theorem 1.1. Then

$$
\begin{aligned}
\left\|\alpha^{\prime}(t)\right\|_{1,1-\mu, \Omega} \leq \varphi(A(0, t))\left[d_{1}\left(t_{0}\right)+\left\|w\left(t_{0}\right)\right\|_{1,0, \Omega}+\left|w\left(t_{0}\right)\right|_{2,1, \Omega}\right] & +c \gamma_{2} e^{-\nu_{2}\left(t-t_{0}\right)} \\
0 & <t_{0}<t \leq T
\end{aligned}
$$

where $\alpha^{\prime}=\left(\alpha_{1}, \alpha_{3}\right)$ and $d_{1}(t)$ is defined by (8.40).

Proof. Let us introduce a smooth function $\zeta=\zeta(t)$ such that $\zeta(t)=0$ for $t<t_{1}$ and $\zeta(t)=1$ for $t>t_{2}$. Multiplying (1.7) by $\zeta$ and introducing the notation $\widetilde{\alpha}^{\prime}=\alpha^{\prime} \zeta$ we obtain

$$
\begin{array}{lc}
\widetilde{\alpha}_{1, t}+v \cdot \nabla \widetilde{\alpha}_{1}-\widetilde{\alpha}_{1} v_{r, r}-\frac{\widetilde{\alpha}_{2}}{r} h_{r}-\widetilde{\alpha}_{3} v_{r, z}+\frac{2 \nu}{r^{2}}\left(h_{r, z}-h_{z, r}\right) \zeta & \\
+\frac{\nu \widetilde{\alpha}_{1}}{r^{2}}-\nu \Delta \widetilde{\alpha}_{1}=\widetilde{F}_{1}+\dot{\zeta} \alpha_{1} & \text { in } \Omega^{T}, \\
\widetilde{\alpha}_{3, t}+v \cdot \nabla \widetilde{\alpha}_{3}-\left(\widetilde{\alpha}_{1} v_{z, r}+\widetilde{\alpha}_{3} v_{z, z}\right)-\frac{\widetilde{\alpha}_{2}}{r} h_{z}-\nu \Delta \widetilde{\alpha}_{3}=\widetilde{F}_{3}+\dot{\zeta} \alpha_{3} & \text { in } \Omega^{T}, \\
a_{2} \widetilde{\alpha}_{1}-a_{1} \widetilde{\alpha}_{3}=-\frac{2 a_{1}}{r} \widetilde{w} & \text { on } S^{T}, \\
\left(a_{1} \widetilde{\alpha}_{1}+a_{2} \widetilde{\alpha}_{3}\right)_{, n}=\beta_{1} \widetilde{h}_{r}+\beta_{2} \widetilde{h}_{z}+\beta_{3} \widetilde{w}_{, r}+\beta_{4} \widetilde{w}_{, z}+\beta_{5} \widetilde{w} & \text { on } S^{T}, \\
\left.\widetilde{\alpha}_{1}\right|_{t=0}=0,\left.\quad \widetilde{\alpha}_{3}\right|_{t=0}=0 & \text { in } \Omega,
\end{array}
$$

where $\dot{\zeta}=\partial_{t} \zeta$. Applying Lemma 4.3 (see (4.12)) to problem (8.34) yields

$$
\begin{aligned}
\left\|\alpha^{\prime}(t)\right\|_{1,1-\mu, \Omega} \leq & c \sup _{t_{1} \leq t^{\prime} \leq t}\left|\widetilde{\alpha}^{\prime}\left(t^{\prime}\right)\right|_{2,1-\mu, \Omega} \varphi(A(0, t)) \\
& +c\left|\widetilde{\alpha}_{2} / r\right|_{10 / 3, \Omega \times\left(t_{1}, t\right)}\|h\|_{2,-\mu, \Omega \times\left(t_{1}, t\right)} \\
& +c\left(\|h\|_{2,-\mu, \Omega \times\left(t_{1}, t\right)}+\|w\|_{2,1-\mu, \Omega \times\left(t_{1}, t\right)}\right) \\
& +c\left|\widetilde{\alpha}_{1}\right|_{2,-(1+\mu), \Omega \times\left(t_{1}, t\right)}+c\left|F^{\prime}\right|_{2,1-\mu, \Omega \times\left(t_{1}, t\right)} \\
& +\frac{c}{t_{2}-t_{1}}\left|\alpha^{\prime}\right|_{2,1-\mu, \Omega \times\left(t_{1}, t\right)}
\end{aligned}
$$

for all $\mu \in(1 / 2,1)$, where $\alpha^{\prime}=\left(\alpha_{1}, \alpha_{3}\right), \widetilde{\alpha}^{\prime}=\left(\widetilde{\alpha}_{1}, \widetilde{\alpha}_{3}\right)$. From (4.14) we have

$$
\begin{aligned}
\sup _{t_{1} \leq t^{\prime} \leq t}\left|\widetilde{\alpha}^{\prime}\left(t^{\prime}\right)\right|_{2, \Omega} \leq & \varphi(A(0, t))\left[\left|\widetilde{\alpha}_{2} / r\right|_{10 / 3, \Omega \times\left(t_{1}, t\right)}\|h\|_{2,-1, \Omega \times\left(t_{1}, t\right)}\right. \\
& \left.+\|w\|_{2,1-\mu, \Omega \times\left(t_{1}, t\right)}+\|h\|_{2,-1, \Omega \times\left(t_{1}, t\right)}+\left|F^{\prime}\right|_{2, \Omega \times\left(t_{1}, t\right)}\right] \\
& +\frac{c}{t_{2}-t_{1}}\left|\alpha^{\prime}\right|_{2, \Omega \times\left(t_{1}, t\right)} .
\end{aligned}
$$


From (4.1) we get

$$
\begin{aligned}
& \left|\widetilde{\alpha}_{2} / r\right|_{10 / 3, \Omega^{t}} \leq c\left(\sup _{t}\left|\widetilde{\alpha}_{2}\right|_{2,-1, \Omega}+\left(\int_{0}^{t}\left|\nabla\left(\widetilde{\alpha}_{2} / r\right)\right|_{2, \Omega}^{2} d t^{\prime}\right)^{1 / 2}\right) \\
& \leq \varphi(A(0, t))\left(\|h\|_{2,-1, \Omega \times\left(t_{1}, t\right)}+\left|v_{r}\right|_{10 / 3, \Omega \times\left(t_{1}, t\right)}+1\right) \delta_{0} \\
& \quad+c\left[\left(1+\sup _{t_{1} \leq t^{\prime} \leq t}\left\|w\left(t^{\prime}\right)\right\|_{1,0, \Omega}\right)\|h\|_{2,-1, \Omega \times\left(t_{1}, t\right)}+\sup _{t_{1} \leq t^{\prime} \leq t}\left\|w\left(t^{\prime}\right)\right\|_{1,0, \Omega}\right. \\
& \left.\quad \cdot\left(\int_{0}^{t}\left|\widetilde{\alpha}_{1}\left(t^{\prime}\right)\right|_{4,-3 / 4-\varepsilon, \Omega} d t^{\prime}\right)^{1 / 2}+\left|F_{\varphi}\right|_{2,-1, \Omega \times\left(t_{1}, t\right)}\right]+\frac{c}{t_{2}-t_{1}}\left|\alpha_{2}\right|_{2,-1, \Omega \times\left(t_{1}, t\right)} .
\end{aligned}
$$

To estimate the second factor in the third term on the r.h.s. of (8.37) we use inequality (4.37) for solutions of problem (8.34). Hence we have

$$
\begin{aligned}
&\left(\int_{0}^{t}\left|\widetilde{\alpha}_{1}\left(t^{\prime}\right)\right|_{4,-3 / 4-\varepsilon, \Omega}^{2} d t^{\prime}\right)^{1 / 2} \leq c\left(\int_{0}^{t}\left\|\widetilde{\alpha}_{1}\left(t^{\prime}\right)\right\|_{1,-\mu, \Omega}^{2} d t^{\prime}\right)^{1 / 2} \\
& \leq \varphi(A(0, t))\left[\left|\widetilde{\alpha}_{2} / r\right|_{10 / 3, \Omega^{t}}\|h\|_{2,-1, \Omega \times\left(t_{1}, t\right)}+\|h\|_{2,-1, \Omega \times\left(t_{1}, t\right)}\right. \\
&+\|w\|_{2,1-\mu, \Omega \times\left(t_{1}, t\right)}+\left|F^{\prime}\right|_{2, \Omega \times\left(t_{1}, t\right)}+\left|F_{1}\right|_{2,-\mu, \Omega \times\left(t_{1}, t\right)} \\
&\left.+\frac{1}{t_{2}-t_{1}}\left|\alpha^{\prime}\right|_{2,-\mu, \Omega \times\left(t_{1}, t_{2}\right)}\right]
\end{aligned}
$$

for any $\mu \in(1 / 2,1)$. Inserting (8.38) in (8.37) and assuming that $\bar{X}$ is sufficiently small we obtain

$$
\begin{aligned}
\left|\widetilde{\alpha}_{2} / r\right|_{10 / 3, \Omega^{t}} & \leq c\left(\sup _{t}\left|\widetilde{\alpha}_{2}\right|_{2,-1, \Omega}+\left(\int_{0}^{t}\left|\nabla\left(\widetilde{\alpha}_{2} / r\right)\right|_{2, \Omega}^{2} d t^{\prime}\right)^{1 / 2}\right) \\
\leq & \varphi(A(0, t))\left(\|h\|_{2,-1, \Omega \times\left(t_{1}, t\right)}+\left|v_{r}\right|_{10 / 3, \Omega \times\left(t_{1}, t\right)}+1\right) \delta_{0} \\
& +c\left(1+\sup _{t_{1} \leq t^{\prime} \leq t}\left\|w\left(t^{\prime}\right)\right\|_{1,0, \Omega}\right)\|h\|_{2,-1, \Omega \times\left(t_{1}, t\right)} \\
& +\varphi(A(0, t)) \sup _{t_{1} \leq t^{\prime} \leq t}\left\|w\left(t^{\prime}\right)\right\|_{1,0, \Omega}\left[\|h\|_{2,-1, \Omega \times\left(t_{1}, t\right)}+\|w\|_{2,1-\mu, \Omega \times\left(t_{1}, t\right)}\right. \\
& \left.+\left|F^{\prime}\right|_{2, \Omega \times\left(t_{1}, t\right)}+\left|F_{1}\right|_{2,-\mu, \Omega \times\left(t_{1}, t\right)}+\frac{1}{t_{2}-t_{1}}\left|\alpha^{\prime}\right|_{2,-\mu, \Omega \times\left(t_{1}, t_{2}\right)}\right] \\
& +c\left|F_{\varphi}\right|_{2,-1, \Omega \times\left(t_{1}, t\right)}+\frac{c}{t_{2}-t_{1}}\left|\alpha_{2}\right|_{2,-1, \Omega \times\left(t_{1}, t\right)} .
\end{aligned}
$$

Let us introduce

$$
d_{1}(t)=\left|h_{, t}(t)\right|_{2, \Omega}+\|h(t)\|_{1,-1, \Omega}+\gamma(t)+\gamma_{1}(t) .
$$

Assume the decay estimate

$$
\gamma_{2}(t) \equiv\left|F^{\prime}(t)\right|_{2,1-\mu, \Omega}+\left|F^{\prime}(t)\right|_{2, \Omega}+\left|F_{1}(t)\right|_{2,-\mu, \Omega} \leq \gamma_{2}(0) e^{-\nu_{2} t}
$$

From (6.49) we have

$$
\|h\|_{2,-1, \Omega^{t}} \leq \varphi(A(0, t)) \bar{X} \quad \text { so } \quad\|h\|_{2,-1, \Omega^{t}} \leq \varphi(A(0, t)) d_{1}(0)
$$


and (6.62) implies

$$
\|w\|_{2,1-\mu, \Omega^{t}} \leq \varphi(A(0, t)) \bar{X}
$$

In view of (8.40) inequality (8.8) takes the form

$$
\|w(t)\|_{1,0, \Omega} \leq \varphi(A(0, t))\left[|w(0)|_{2,1, \Omega}+d_{1}(t)+e^{-t}\|w(0)\|_{1,0, \Omega}+e^{-\nu t}|w(0)|_{2, \Omega}\right] .
$$

Applying the above estimates in (8.39) and using the fact that $\bar{X}$ is sufficiently small we obtain

$$
\begin{aligned}
\left|\widetilde{\alpha}_{2} / r\right|_{10 / 3, \Omega^{t}} & \leq c\left[\sup _{t}\left|\widetilde{\alpha}_{2}\right|_{2,-1, \Omega}+\left(\int_{0}^{t}\left|\nabla\left(\widetilde{\alpha}_{2} / r\right)\right|^{2} d t^{\prime}\right)^{1 / 2}\right] \\
\leq & \varphi(A(0, t))\left(\left|v_{r}\right|_{10 / 3, \Omega \times\left(t_{1}, t\right)}+1\right) \delta_{0} \\
& +\varphi(A(0, t))\left[\left|w\left(t_{0}\right)\right|_{2,1, \Omega}+d_{1}\left(t_{0}\right)+\gamma_{2}\left(t_{0}\right)+e^{-\left(t-t_{0}\right)}\left\|w\left(t_{0}\right)\right\|_{1,0, \Omega}\right. \\
& \left.+e^{-\nu\left(t-t_{0}\right)}\left|w\left(t_{0}\right)\right|_{2, \Omega}\right]+c\left|F_{\varphi}\right|_{2,-1, \Omega \times\left(t_{0}, t\right)}+\frac{c}{t_{2}-t_{1}}\left|\alpha_{2}\right|_{2,-1, \Omega \times\left(t_{1}, t_{2}\right)}
\end{aligned}
$$

for some $t_{0}<t_{1}$. From Lemma 3.4 we have

$$
|v(t)|_{2, \Omega} \leq|f|_{2,1, \Omega^{t}}+|v(0)|_{2, \Omega},
$$

and

$$
\left|v^{\prime}\right|_{10 / 3, \Omega^{t}} \leq c\left(\sup _{t}\left|v^{\prime}(t)\right|_{2, \Omega}^{2}+\left|\nabla v^{\prime}\right|_{2, \Omega^{t}}^{2}\right)^{1 / 2} \leq c\left(|f|_{2,1, \Omega^{t}}+|v(0)|_{2, \Omega}\right),
$$

where $v^{\prime}$ is defined in the proof of Lemma 3.4.

Inserting (8.11) and (8.47) in (8.36) (where (8.45) is employed) yields

$$
\begin{aligned}
& \sup _{t_{1} \leq t^{\prime} \leq t}\left|\alpha^{\prime}\left(t^{\prime}\right)\right|_{2, \Omega} \leq \varphi(A(0, t)) \delta_{0}\left(|f|_{2,1, \Omega^{t}}+|v(0)|_{2, \Omega}+1\right) d_{1}\left(t_{0}\right) \\
&+\varphi(A(0, t))\left[\left|w\left(t_{0}\right)\right|_{2,1, \Omega}+d_{1}\left(t_{0}\right)+e^{-\left(t-t_{0}\right)}\left\|w\left(t_{0}\right)\right\|_{1,0, \Omega}\right] \\
&+\varphi(A(0, t))\left[\left|F_{\varphi}\right|_{2,-1, \Omega \times\left(t_{0}, t\right)}+\frac{1}{t_{2}-t_{1}}\left|\alpha_{2}\right|_{2,-1, \Omega \times\left(t_{0}, t\right)}\right] d_{1}\left(t_{0}\right) \\
&+\varphi(A(0, t))\left[\|w\|_{2,1-\mu, \Omega \times\left(t_{0}, t\right)}+\left|F^{\prime}\right|_{2, \Omega \times\left(t_{0}, t\right)}+\frac{c}{t_{2}-t_{1}}\left|\alpha^{\prime}\right|_{2, \Omega \times\left(t_{1}, t\right)}\right] .
\end{aligned}
$$

From (6.62) we have

$$
\begin{gathered}
\|w\|_{2,1-\mu, \Omega^{t} \leq} \varphi(A(0, t))\left[\|h(0)\|_{1,-1, \Omega}+\|w(0)\|_{1,0, \Omega}\right. \\
\left.+\gamma(0) e^{-\nu t}+\gamma_{1}(0) e^{-\nu_{1} t}\right] .
\end{gathered}
$$

Employing (8.49) and (6.81) in the form

$$
\left\|\alpha^{\prime}\right\|_{2,1-\mu, \Omega^{t}} \leq \varphi(A(0, t), B(0, t)) \bar{X}
$$

we obtain from (8.48) the inequality

$$
\begin{aligned}
\sup _{t_{1} \leq t^{\prime} \leq t}\left|\widetilde{\alpha}^{\prime}\left(t^{\prime}\right)\right|_{2, \Omega} \leq & \varphi(A(0, t), B(0, t))\left[d_{1}\left(t_{0}\right)+\left\|w\left(t_{0}\right)\right\|_{1,0, \Omega}\right. \\
& \left.+\left|w\left(t_{0}\right)\right|_{2,1, \Omega}+\gamma_{2}\left(t_{0}\right)\right]+\frac{c}{t_{2}-t_{1}}\left|\alpha^{\prime}\right|_{2, \Omega \times\left(t_{1}, t\right)} .
\end{aligned}
$$


From (3.5) we have

$$
\begin{aligned}
&\left|\alpha^{\prime}\right|_{2, \Omega \times\left(t_{0}, t\right)}+\left|\alpha_{1}\right|_{2,-(1+\mu), \Omega \times\left(t_{0}, t\right)} \leq \varphi(A(0, t))\left|t-t_{0}\right|^{1 / 2} \\
& \cdot\left[d_{1}\left(t_{0}\right)+\left|w\left(t_{0}\right)\right|_{2,1, \Omega}+e^{-\nu\left(t-t_{0}\right)}\left\|w\left(t_{0}\right)\right\|_{1,0, \Omega}\right] .
\end{aligned}
$$

Making use of the above estimates in (8.35) yields

$$
\left\|\alpha^{\prime}(t)\right\|_{1,1-\mu, \Omega} \leq \varphi(A(0, t))\left[d_{1}\left(t_{0}\right)+\left\|w\left(t_{0}\right)\right\|_{1,0, \Omega}+\left|w\left(t_{0}\right)\right|_{2,1, \Omega}\right]+c \gamma_{2} e^{-\nu_{2}\left(t-t_{0}\right)} .
$$

This ends the proof.

\subsection{Proof of global existence}

Proof of Theorem 1.2. To show $(8.4)_{1}$ for $i=1$ we collect the necessary estimates. Since

$$
\left|h_{, t}(t)\right|_{2, \Omega}+\|h(t)\|_{1,-1, \Omega} \leq \varphi(A(0, t)) e^{-\nu t_{0}}\left(\left|h_{, t}(0)\right|_{2, \Omega}+\|h(0)\|_{1,-1, \Omega}+\gamma(0)\right)
$$

for some $t_{0}<t$, we have

$$
d_{1}(t) \leq d_{1}(0) e^{-\nu_{*} t_{0}}, \quad \nu_{*}=\min \left\{\nu_{0}, \nu_{1}\right\} .
$$

From (8.8) we get

$$
\|w(t)\|_{1,0, \Omega} \leq \varphi(A(0, t))\left[|w(0)|_{2,1, \Omega}+d_{1}(0)+e^{-\nu t}\|w(0)\|_{1,0, \Omega}\right] .
$$

Next (8.17) implies

$$
|w(t)|_{2,1, \Omega} \leq|w(0)|_{2,1, \Omega}+\varphi(A(0, t)) d_{1}(0) \equiv d_{*},
$$

which holds for all $t \leq \infty$ if $A(0, t)$ does not increase with time. In view of (8.54)-(8.57) the inequality (8.53) takes the form

$$
\begin{aligned}
\left\|\alpha^{\prime}(t)\right\|_{1,1-\mu, \Omega} \leq & \varphi(A(0, t))\left[e^{-\nu_{*} t_{0}} d_{1}(0)+d_{1}(0)\right. \\
& \left.+e^{-\nu t}\|w(0)\|_{1,0, \Omega}\right]+c \gamma_{2}(0) e^{-\nu_{2} t}, \quad t \leq T,
\end{aligned}
$$

where $T$ is the time of local existence.

Assume that

$$
\|w(0)\|_{1,0, \Omega} \leq A_{1}
$$

where $A_{1}$ is a constant so large that

$$
\varphi(A(0, T))\left[d_{*}+d_{1}(0)+e^{-\nu T} A_{1}\right] \leq A_{1} .
$$

Then

$$
\|w(T)\|_{1,0, \Omega} \leq A_{1} .
$$

Moreover,

$$
d_{1}(T) \leq d_{1}(0) .
$$

Inserting (8.59) in (8.58) yields

$$
\begin{aligned}
\left\|\alpha^{\prime}(T)\right\|_{1,1-\mu, \Omega} \leq & \varphi(A(0, T))\left[e^{-\nu_{*} t_{0}} d_{1}(0)+d_{1}(0)+e^{-\nu T} A_{1}\right] \\
& +c \gamma_{2}(0) e^{-\nu T} \equiv A_{2} .
\end{aligned}
$$

Hence we have

$$
\begin{aligned}
\left\|\alpha^{\prime}(2 T)\right\|_{1,1-\mu, \Omega} & \leq \varphi(A(t, 2 T))\left[e^{-\nu_{*} t_{0}} d_{1}(T)+d_{1}(T)+e^{-\nu T} A_{1}\right]+c \gamma_{2}(T) e^{-\nu T} \\
& \leq \varphi(A(0, T))\left[e^{-\nu_{*} t_{0}} d_{1}(0)+d_{1}(0)+e^{-\nu T} A_{1}\right]+c \gamma_{2}(0) e^{-\nu T}=A_{2}
\end{aligned}
$$


In this way we have shown that there exists $Q_{1}^{*}<\infty$ such that

$$
Q_{1}(t) \leq Q_{1}^{*} \quad \forall t \in \mathbb{R}_{+} .
$$

From (8.25) we have the estimate

$$
\begin{aligned}
|\chi(t)|_{2,-1, \Omega} \leq & \varphi(A(0, t))\left[|w(0)|_{2,1, \Omega}+d_{1}(0)+e^{-t}\|w(0)\|_{1,0, \Omega}\right] \\
& +c\left|F_{\varphi}\right|_{2,-1, \Omega^{t}}+c \delta_{0} A(0, t)+e^{-\nu t}|\chi(0)|_{2,-1, \Omega} .
\end{aligned}
$$

Using the growth condition

$$
\gamma_{3}(t) \equiv\left|F_{\varphi}(t)\right|_{2,-1, \Omega} \leq \gamma_{3}(0) e^{-\nu_{3} t}
$$

we obtain from (8.64) the inequality

$$
\begin{aligned}
|\chi(t)|_{2,-1, \Omega} \leq & \varphi(A(0, t))\left[|w(0)|_{2,1, \Omega}+d_{1}(0)+e^{-t}\|w(0)\|_{1,0, \Omega}\right] \\
& +c \gamma_{3}(0)+c \delta_{0} A(0, t)+e^{-\nu t}|\chi(0)|_{2,-1, \Omega} .
\end{aligned}
$$

From (8.45) we have

$$
\begin{aligned}
&|\tilde{\chi} / r|_{10 / 3, \Omega^{t}} \leq c\left(|\tilde{\chi}|_{2,-1, \Omega}+\left(\int_{0}^{t}|\nabla(\tilde{\chi} / r)|_{2, \Omega}^{2} d t^{\prime}\right)^{1 / 2}\right) \\
& \leq \varphi(A(0, t))\left[\left(\left|v_{r}\right|_{10 / 3, \Omega^{t}}+1\right) \delta_{0}+\left|w\left(t_{0}\right)\right|_{2,1, \Omega}+d_{1}\left(t_{0}\right)+\gamma_{2}\left(t_{0}\right)\right. \\
&\left.+e^{-\left(t-t_{0}\right)}\left\|w\left(t_{0}\right)\right\|_{1,0, \Omega}+\gamma_{3}\left(t_{0}\right)\right] \\
&+\frac{c}{\left(t_{2}-t_{1}\right)^{1 / 2}}\left\{\varphi(A(0, t))\left[\left|w\left(t_{0}\right)\right|_{2,1, \Omega}+d_{1}\left(t_{0}\right)+e^{-\left(t-t_{0}\right)}\left\|w\left(t_{0}\right)\right\|_{1,0, \Omega}\right]\right. \\
&\left.+\gamma_{2}\left(t_{0}\right)+\gamma_{3}\left(t_{0}\right)+\delta_{0} A(0, t)+e^{-\nu\left(t-t_{0}\right)}\left|\chi\left(t_{0}\right)\right|_{2,-1, \Omega}\right\} .
\end{aligned}
$$

Finally, (8.67) implies

$$
\begin{aligned}
|\tilde{\chi} / r|_{10 / 3, \Omega^{t}} \leq & c\left[\sup _{t_{0}<t}|\tilde{\chi}|_{2,-1, \Omega}+\left(\int_{0}^{t}|\nabla(\tilde{\chi} / r)|_{2, \Omega} d t^{\prime}\right)^{1 / 2}\right] \\
\leq & \varphi(A(0, t))\left[\delta_{0}+\left|w\left(t_{0}\right)\right|_{2,1, \Omega}+d_{2}\left(t_{0}\right)+\gamma_{3}\left(t_{0}\right)\right. \\
& \left.+e^{-\left(t-t_{0}\right)}\left\|w\left(t_{0}\right)\right\|_{1,0, \Omega}+e^{-\nu\left(t-t_{0}\right)}\left|\chi\left(t_{0}\right)\right|_{2,-1, \Omega}\right],
\end{aligned}
$$

where

$$
d_{2}(t)=d_{1}(t)+\gamma_{2}(t)
$$

From (4.49) we have

$$
\begin{aligned}
&\|\chi(t)\|_{1,1-\mu, \Omega} \leq\|\widetilde{\chi}\|_{2,1-\mu, \Omega^{t}} \\
& \leq {\left[\varepsilon_{1}\|v\|_{A_{1-\mu}\left(\Omega^{t}\right)}+c\left(1 / \varepsilon_{1}\right)\left(\gamma_{4}\left(t_{0}\right)+|v(0)|_{2, \Omega}\right)+c\|v(0)\|_{2,2,1-\mu, \Omega}+1\right] } \\
& \cdot\left[\sup _{t}|\tilde{\chi} / r|_{2, \Omega}+\left(\int_{0}^{t}|\nabla(\tilde{\chi} / r)|_{2, \Omega}^{2} d t^{\prime}\right)^{1 / 2}\right] \\
&+\varphi(A(0, t)) \sup _{t_{0} \leq t}\|w\|_{1,0, \Omega}+\gamma_{3}\left(t_{0}\right) \\
&+\left[\varepsilon(A(0, t))+c(1 / \varepsilon)\left(\gamma_{4}\left(t_{0}\right)+|v(0)|_{2, \Omega}\right)\right] \delta_{0}+\frac{c}{t_{2}-t_{1}}|\chi|_{2,1-\mu, \Omega \times\left(t_{1}, t_{2}\right)},
\end{aligned}
$$


where

$$
\gamma_{4}(t) \equiv|f(t)|_{2, \Omega} \leq \gamma_{4}(0) e^{-\nu_{4} t}
$$

In view of (8.8) and (8.68) we obtain from (8.69) the inequality

$$
\begin{aligned}
\|\chi(t)\|_{1,2,1-\mu, \Omega} \leq & \varphi(A(0, t))\left[d_{3}\left(t_{0}\right)+|v(0)|_{2, \Omega}+\left|w\left(t_{0}\right)\right|_{2,1, \Omega}\right. \\
& \left.+\delta_{0}+e^{-\left(t-t_{0}\right)}\left\|w\left(t_{0}\right)\right\|_{1,0, \Omega}+e^{-\nu\left(t-t_{0}\right)}\left|\chi\left(t_{0}\right)\right|_{2,-1, \Omega}\right],
\end{aligned}
$$

where

$$
d_{3}(t)=d_{2}(t)+\gamma_{3}(t)+\gamma_{4}(t) .
$$

Next we examine $|\chi(t)|_{2,-1, \Omega}$ from (8.66). Assume that $|\chi(0)|_{2,-1, \Omega} \leq A_{3}$. Then (8.66) implies

$$
\begin{aligned}
|\chi(T)|_{2,-1, \Omega} \leq & \varphi(A(0, T))\left[d_{*}+d_{3}(T)+e^{-T} A_{1}\right]+c \gamma_{3}(T) \\
& +c \delta_{0} A(0, T)+e^{-\nu T} A_{3} \leq A_{3},
\end{aligned}
$$

where the second inequality follows for $A_{3}$ sufficiently large. Inequality (8.71) can be repeated step by step because $d_{*}$ is fixed for all $t, d_{3}(t)$ is a decreasing function and $e^{-T} A_{1} \leq A_{1}, e^{-\nu T} A_{2} \leq A_{2}$.

Since $|\chi(t)|_{2,-1, \Omega}$ does not increase with time we have the same for $\|\chi(t)\|_{1,1-\mu, \Omega}$.

These considerations imply global existence for problem (1.1). Hence Theorem 1.2 is proved.

Theorem 1.3 follows directly from Theorems 1.1 and 1.2. 


\section{Historical overview}

In this section we recall results concerning the existence and regularity of solutions to the Navier-Stokes equations.

9.1. Problems with slip boundary conditions. In this subsection we concentrate on problem (1.1) under different geometrical and analytical restrictions, where the slip boundary conditions are employed. The boundary conditions imply the boundary conditions for vorticity, so a problem for vorticity can be considered. Then applying the ideas of [lad 1] and [uky] the global estimate for vorticity modulo norms of small quantities follows. This is the main step for proofs of global existence of solutions to problems (1.1).

In this paper we proved existence of global regular solutions to problem (1.1) assuming that $w(0), h(0), f_{\varphi}, F_{r}, F_{z}$ are small and imposing decay of the external force (see (1.21)). The results are formulated in Theorems 1.1-1.3. In this paper $\Omega$ is an axially symmetric domain. Hence to show global existence a lot of strong geometrical restrictions on the boundary must be imposed (see assumptions A.1, A.2, A.3). Most of them follow from the fact that $\left.\chi\right|_{S} \neq 0$, which is implied by the fact that $\Omega$ is axially symmetric but noncylindrical and the slip coefficient $\gamma$ is nonvanishing.

In [zaj 5] problem (1.1) in a cylinder and with vanishing slip coefficient $\gamma$ is considered. In this case $\left.\chi\right|_{S}=0$, so the proofs of the results similar to Theorems 1.1-1.3 are much simpler. The ideas of the proofs are the same as in this paper.

Since in this paper and in [zaj 5] we proved existence of solutions to (1.1) with small azimuthal component of velocity $v_{\varphi}$ we are interested to have the existence of solutions with large $v_{\varphi}$. In [zaj 7] existence of global axially symmetric solutions with large swirl is proved in a cylinder with the axis of symmetry removed. We do not know how to obtain any estimate for $v_{\varphi}$ near the axis of symmetry without assuming that $v_{\varphi}$ is sufficiently small. This is connected with the property that any axially symmetric solution near the axis of symmetry behaves as a three-dimensional one (see [zaj 11]).

Existence of global solutions with large swirl which are close to the axially symmetric solutions from [zaj 7] is also proved in [zaj 8] for cylinders with the axis of symmetry removed.

In [zaj 11] we showed existence of global axially symmetric solutions in a full cylinder which are such that near the axis of symmetry $v_{\varphi}$ is sufficiently small but at a sufficiently large distance from it $v_{\varphi}$ is large. The existence follows from the Leray-Schauder fixed point theorem by making use of an appropriate partition of unity.

In this paper and in $[\mathrm{zaj} 5,7,8,11,12]$ the existence of solutions which are either axially symmetric or close to axially symmetric and in axially symmetric domains is proved. 
In [zaj 9-10] we showed the existence of solutions in non-axially symmetric cylinders. The solutions are such that they do not change much with respect to the variable along the cylinder. Hence they are close to two-dimensional solutions. The papers generalize the results of Raugel-Sell (see [ras 1-3]). The existence is proved via the Leray-Schauder fixed point theorem. In [zaj 10, 12] the case with inflow and outflow, while in [zaj 9] the case without inflow and outflow are considered.

9.2. Local existence and uniqueness of strong solutions. We shall start with the most fundamental problem: existence and uniqueness of local strong solutions for different initial-boundary value problems. We indicate different approaches and relations between them.

To present results concerning problems (1.1)-(1.3) we first introduce some function spaces (the notation concerning the results of this paper is introduced in Chapter 2). By $C_{0, \sigma}^{\infty}(\Omega)$ we denote the set of all $C^{\infty}$ vector functions $\varphi=\left(\varphi_{1}, \ldots, \varphi_{n}\right)$ with compact support in $\Omega$ such that $\operatorname{div} \varphi=0$, where $\Omega \subseteq \mathbb{R}^{n} . L_{r, \sigma}(\Omega)$ is the closure of $C_{0, \sigma}^{\infty}(\Omega)$ with respect to the $L_{r}$-norm, $(\cdot, \cdot)$ denotes the duality pairing between $L_{r}(\Omega)$ and $L_{r^{\prime}}(\Omega)$, where $1 / r+1 / r^{\prime}=1$, and

$$
\|u\|_{L_{r}(\Omega)}=\left(\int_{\Omega}|u(x)|^{r} d x\right)^{1 / r},
$$

where $u$ is a scalar or vector-valued function. $H_{0, \sigma}^{s}(\Omega)$ denotes the closure of $C_{0, \sigma}^{\infty}(\Omega)$ with respect to the norm

$$
\|u\|_{H_{0, \sigma}^{s}(\Omega)}=\sum_{|\alpha| \leq s}\left\|D_{x}^{\alpha} u\right\|_{L_{2, \sigma}(\Omega)} .
$$

For an interval $I \subset \mathbb{R}^{1}$ and a Banach space $X, L_{p}(I ; X)$ and $C^{m}(I ; X)$ denote the usual Banach spaces of functions on $I$ with values in $X$, respectively, where $p \in[1, \infty]$ and $m=0,1, \ldots$

Now we define weak solutions to problems $(1.1)_{1,2,5}$ and $(1.1)_{1,2,5},(1.3)$.

Definition 9.2.1. Let $v(0) \in L_{2, \sigma}(\Omega)$. Let $\Omega \subseteq \mathbb{R}^{n}, n=2,3$. A measurable function $v$ on $\Omega^{T}=\Omega \times(0, T)$ is called a weak solution to problems $(1.1)_{1,2,5}$ and $(1.1)_{1,2,5},(1.3)$ if

(1) $v \in L_{\infty}\left(0, T ; L_{2, \sigma}(\Omega)\right) \cap L_{2}\left(0, T ; H_{0, \sigma}^{1}(\Omega)\right)$;

(2) for every $\varphi \in H^{1}\left(0, T ; H_{0, \sigma}^{1}(\Omega) \cap L_{n}(\Omega)\right)$ with $\varphi(T)=0$,

$$
\int_{0}^{T}[-(v, \varphi, t)+\nu(\nabla v, \nabla \varphi)+(v \cdot \nabla v, \varphi)] d t=(v(0), \varphi(0)) .
$$

Concerning existence of weak solutions, by Leray [ler] and Hopf [hop] we have

Theorem 9.2.2 (Leray-Hopf). For every $v(0) \in L_{2, \sigma}(\Omega)$, there exists at least one weak solution to the Cauchy problem $(1.1)_{1,2,5}$ (see [ler]) and to the Dirichlet problem $(1.1)_{1,2,5}$, (1.3) (see $[\mathrm{hop}])$ for $t \in(0, \infty)$ such that

$$
|v(t)|_{2, \Omega}^{2}+2 \nu \int_{0}^{t}\left|\nabla v\left(t^{\prime}\right)\right|_{2, \Omega}^{2} d t^{\prime} \leq|v(0)|_{2, \Omega}^{2}
$$


and

$$
|v(t)-v(0)|_{2, \Omega} \rightarrow 0 \quad \text { as } t \rightarrow+0
$$

(see notation in Section 2).

Let us now consider problem (1.1).

DeFinition 9.2.3. By a weak solution to problem (1.1) we mean a function $v \in L_{\infty}(0, T$; $\left.L_{2, \sigma}(\Omega)\right) \cap L_{2}\left(0, T ; H_{\sigma}^{1}(\Omega)\right)$ satisfying the integral identity

$$
\begin{aligned}
\int_{0}^{T}\left[-\left(v, \varphi_{, t}\right)+\nu(\mathbb{D}(v), \mathbb{D}(\varphi))+\gamma\left(v \cdot \bar{\tau}_{\alpha}, \varphi \cdot \bar{\tau}_{\alpha}\right)_{S}+\right. & (v \cdot \nabla v, \varphi)] d t \\
& =(v(0), \varphi(0))+\int_{0}^{T}(f, v) d t
\end{aligned}
$$

which holds for any $\varphi \in H^{1}\left(0, T ; H_{\sigma}^{1}(\Omega)\right)$ with $\varphi(T)=0$, where $\left(v \cdot \bar{\tau}_{\alpha}, \varphi \cdot \bar{\tau}_{\alpha}\right)_{S}=$ $\int_{S} v \cdot \bar{\tau}_{\alpha} \varphi \cdot \bar{\tau}_{\alpha} d S$ and the summation convention over the repeated index $\alpha$ is assumed.

By Lemma 3.5 the following estimate for weak solutions satisfying (9.2.4) holds:

$$
|v(t)|_{2, \Omega}^{2}+\nu \int_{0}^{t}\left|\nabla v\left(t^{\prime}\right)\right|_{2, \Omega}^{2} d t^{\prime} \leq c(1+t)\left(|f|_{2,1, \Omega^{t}}^{2}+|v(0)|_{2, \Omega}^{2}\right) .
$$

Next we recall results on local existence. The first result on the solvability of the Cauchy problem $(1.1)_{1,2,5}$ with initial data $v(0) \in L_{q, \sigma}\left(\mathbb{R}^{n}\right)$ is due to Fabes-Jones-Rivière [fjr]. For this purpose they introduced

Definition 9.2.4. By a very weak solution to problem $(1.1)_{1,2,5}$ we mean a function $v \in L_{r}\left(0, T ; L_{s, \sigma}\left(\mathbb{R}^{n}\right)\right)$ satisfying

$$
\int_{0}^{T}\left[\left(\left(\partial_{t}+\nu \Delta\right) \varphi, v\right)+(\nabla \varphi, v \otimes v)\right] d t=-(\varphi(0), v(0))
$$

for $\varphi \in C_{0, \sigma}^{\infty}\left(\mathbb{R}^{n}\right)$.

Theorem 9.2.5 (Fabes-Jones-Rivière $[\mathrm{fjr}]$ ). Let $v(0) \in L_{q, \sigma}\left(\mathbb{R}^{n}\right)$. Then the Cauchy problem $(1.1)_{1,2,5}$ has a unique local solution $v \in L_{r}\left(0, T ; L_{s, \sigma}\left(\mathbb{R}^{n}\right)\right)$ with $s>n, n / q<$ $2 / r+n / s \leq 1$.

Provided $v(0)$ is sufficiently small in $L_{q}\left(\mathbb{R}^{n}\right) \cap L_{q^{\prime}}\left(\mathbb{R}^{n}\right), 1 / q+1 / q^{\prime}=1$, the solution exists globally, so $T=\infty$.

The existence of local solutions for the Cauchy-Dirichlet problem $(1.1)_{1,2,5}$ with nonhomogeneous boundary conditions (1.3) with data in $L_{p}$ has been proved in $\mathbb{R}_{+}^{n}$ by Lewis (see [lew]) and in a bounded domain by Fabes-Lewis-Rivière [flr].

By different techniques Beirão da Veiga proved (see [bdv 5]):

THEOREM 9.2.6. If $v(0) \in L_{q, \sigma}\left(\mathbb{R}^{n}\right), q>n$, then there exists a unique very weak solution $v$ to problem $(1.1)_{1,2,5}$ which is a weakly continuous function from $[0, T]$ into $L_{q, \sigma}\left(\mathbb{R}^{n}\right)$. Furthermore, if $v(0) \in L_{2, \sigma}\left(\mathbb{R}^{n}\right) \cap L_{q, \sigma}\left(\mathbb{R}^{n}\right)$ then $v \in C\left([0, T] ; L_{2, \sigma}\left(\mathbb{R}^{n}\right) \cap L_{q, \sigma}\left(\mathbb{R}^{n}\right)\right) \cap$ $L_{2}\left((0, T) ; H^{1}\left(\mathbb{R}^{n}\right) \cap L_{q, \sigma}\left(\mathbb{R}^{n}\right)\right)$. 
In addition, Beirão da Veiga gives an estimate for the maximal existence time (depending on $\left.\|v(0)\|_{L_{q}\left(\mathbb{R}^{n}\right)}\right)$ and proves that the solution exists globally, that is, for all time, if the norm $\|v(0)\|_{L_{2}\left(\mathbb{R}^{n}\right) \cap L_{q}\left(\mathbb{R}^{n}\right)}$ is sufficiently small.

More recently C. P. Calderón (see [cal 1-3]) proved also the existence of very weak local solutions to $(1.1)_{1,2,5}$ for $q=n$.

Let $S$ be the Stokes operator $-\nu P \Delta$, where $P$ is the projector onto the solenoidal vector fields. Let $b(v, v)=-P(v \cdot \nabla v)$. Then solutions of problem $(1.1)_{1,2,5}$ satisfy the following integral equation:

$$
v(t)=e^{-t S} v(0)+\int_{0}^{t} e^{-(t-\tau) S}[b(v, v)(\tau)+f(\tau)] d \tau .
$$

Definition 9.2.7. Let $E$ be a Banach space and $I \subset \mathbb{R}_{+}$an interval. By a mild solution (in $E$ ) of $(1.1)_{1,2,5}$ on $I$ we mean a function $v \in C(I ; E)$ satisfying $(9.2 .7)$ on $I$, where $E$ is a Banach space of distributions on which the Stokes semigroup $\left\{e^{-t S}: t \geq 0\right\}$ is strongly continuous and the integral in (9.2.7) is well defined.

Applying the semigroup approach Kato [kat 1] considered Theorem 9.2.6 in the critical case $q=n$. He showed, by using some ideas developed earlier jointly with Fujita (see [fuk 1-2]), the following result:

TheOrem 9.2.8. Given $v(0) \in L_{n, \sigma}\left(\mathbb{R}^{n}\right)$ there exists $T>0$ and a unique solution of $(1.1)_{1,2,5}$ with $f=0$ in the class

$$
C\left([0, T] ; L_{n, \sigma}\left(\mathbb{R}^{n}\right)\right) \cap C^{(1-n / q) / 2}\left((0, T] ; L_{n, \sigma}\left(\mathbb{R}^{n}\right)\right)
$$

for $n<q<\infty$.

We underline that Theorem 9.2.8 is a simplified version of Kato's result (see [can, wie]) since in [kat 1] class (9.2.8) is more restricted.

In [kat 1] it is also shown that $v$ is global if $\|v(0)\|_{L_{n}\left(\mathbb{R}^{n}\right)}$ is sufficiently small.

The case $n=3$ has been extensively studied by Cannone and Meyer [cam] and Cannone [can]. They introduced a concept of "well-suited" spaces for the Navier-Stokes equations. They showed local existence and uniqueness of solutions in the class of wellsuited spaces. In [cam] (see also [can]) it is shown that $L_{q}\left(\mathbb{R}^{n}\right)$ is well-suited if $q>n=3$. In the same paper it is also shown that the Sobolev spaces $H_{2}^{s}\left(\Omega ; \mathbb{R}^{n}\right)$ are well-suited if $s>1 / 2$. Thus, if $n=3, s>1 / 2$ and $v(0) \in H_{2,0, \sigma}^{2}\left(\Omega ; \mathbb{R}^{n}\right)$ then there exists a unique mild solution to the Navier-Stokes equations such that

$$
v \in C\left([0, T] ; H_{2,0, \sigma}^{s}\left(\Omega ; \mathbb{R}^{3}\right)\right),
$$

where

$$
H_{q, 0, \sigma}^{s}\left(\Omega ; \mathbb{R}^{n}\right)= \begin{cases}\left\{u \in H_{q}^{s}\left(\Omega ; \mathbb{R}^{n}\right): \operatorname{div} u=0,\left.u\right|_{\partial \Omega}=0\right\}, & 1 / q<s<2, \\ \left\{u \in H_{q}^{s}\left(\Omega ; \mathbb{R}^{n}\right): \operatorname{div} u=0,\left.u \cdot \bar{n}\right|_{\partial \Omega}=0\right\}, & 0 \leq s<1 / q .\end{cases}
$$

Moreover, the existence time $T$ depends on $\|v(0)\|_{H_{2}^{s}}$ only. This extends an earlier result of Kato [kat 2] who had to suppose that $s>5 / 2$.

The more general case where $v(0)$ belongs to a Bessel potential space $H_{q}^{s}\left(\Omega ; \mathbb{R}^{n}\right)$ has been investigated by Kato-Ponce [kap] for $q \in(1, \infty), s>1+n / q$ if $n=3$, and by Ribaud [rib] for $q \in(1, \infty),-1+n / q<s<(m / q) \wedge(1+n / q) / 2$. Assuming that 
$v(0) \in H_{q, 0, \sigma}^{s}\left(\Omega ; \mathbb{R}^{n}\right)$ they proved that problem $(1.1)_{1,2,5},(1.3)$ has a local mild solution $v \in C\left([0, T) ; H_{q, 0, \sigma}^{s}\left(\Omega ; \mathbb{R}^{n}\right)\right)$. It is unique if $s \geq n(1 / q-1 / 2)_{+}$.

In the situation described above uniqueness is always, except in Ribaud's result, proven under additional restrictions. Moreover, there is no relation between different uniqueness theorems.

Amann [ama] improved the situation. For this purpose he introduced a scale of Besov and Nikol'skii spaces. We recall the simplest result. Let $B_{q, r}^{s}:=B_{q, r}^{s}\left(\Omega ; \mathbb{R}^{n}\right)$ be a Besov space. A little Nikol'skiu space $n_{q}^{s}$ is defined by

$$
n_{q}^{s}:=\text { closure of } H_{q}^{s} \text { in } B_{q, \infty}^{s}, \quad|s| \in(0,2) .
$$

Let us also introduce the spaces $n_{q, 0, \sigma}^{s}:=\left\{u \in n_{q}^{s}: \operatorname{div} u=0\right\}, q \in(1, \infty),|s| \in(0,2)$.

TheOREM 9.2.9. Suppose that $n<q \leq r<\infty$ and $v(0) \in n_{q, 0, \sigma}^{-1+n / q}$. Then there exists a unique maximal solution $v:=v(\cdot, v(0))$ of the Navier-Stokes equations

$$
\begin{aligned}
& v_{, t}+P(v \cdot \nabla v)-\nu P \Delta v=P f, \\
& \operatorname{div} v=0 \\
& \left.v\right|_{S}=0 \\
& \left.v\right|_{t=0}=v(0),
\end{aligned}
$$

such that

$$
v \in C\left(\left(0, t^{+}\right) ; H_{r, 0, \sigma}^{2}\right) \cap C\left(\left(0, t^{+}\right) ; L_{r, \sigma}\right)
$$

and

$$
\lim _{t \rightarrow 0} v(t)=v(0) \quad \text { in } n_{q, 0, \sigma}^{-1+n / q}
$$

as well as

$$
\lim _{t \rightarrow 0} t^{(1-n / q) / 2} v(t)=0 \quad \text { in } L_{q} .
$$

Theorem 9.2.9 guarantees for each $r \geq q$ a unique maximal solution $v_{r}$ on the maximal interval of existence $\left[0, t_{r}^{+}\right)$. Since the spaces (9.2.11) are not comparable for different values of $r$ it is conceivable that $v_{r} \neq v_{s}$ if $r \neq s$.

It has been shown in [ama] that $v_{s} \supset v_{r}$ if $s>r$. This means in particular that $t_{r}^{+} \leq t_{s}^{+}$for $r<s$. Thus, although the solution $v_{r}$ ceases to exist in class (9.2.11) at $t_{r}^{+}$, if $t_{r}^{+}<\infty$, it can be continued to the possibly larger interval $\left[0, t_{s}^{+}\right)$in the class which is obtained by replacing $H_{r, \sigma}^{2}$ and $L_{r, \sigma}$ in $(9.2 .11)$ by $H_{s, \sigma}^{2}$ and $L_{s, \sigma}$, respectively. Thus we should obtain a unique maximal solution $v$, independently of $r>q$, by letting $r \rightarrow \infty$.

In Theorems 9.2.6-9.2.9 local existence of strong solutions is proved. In these theorems under sufficiently small initial data (in corresponding norms) there is always a possibility of prolongation of the local solution with $t \rightarrow \infty$.

Finally, we formulate a local existence result which will be useful to describe the problem of prolongation of local strong solutions.

Theorem 9.2.10 (Kato [kat 1], Giga-Miyakawa [gim], Brezis [bre]). For $n<r<\infty$, there is a constant $\gamma=\gamma(n, r)>0$ such that if the initial data $v(0) \in L_{n, \sigma}\left(\mathbb{R}^{n}\right)$ and 
$T_{*}>0$ satisfy

$$
\sup _{0<t \leq T_{*}} t^{(n / 2)(1 / n-1 / r)}\left\|e^{-t S} v(0)\right\|_{L_{r}\left(\mathbb{R}^{n}\right)}<\gamma
$$

then there exists a unique strong solution $v(t)$ of problem $(1.1)_{1,2,5}$. Moreover, the solution $v$ is such that $t^{(n / 2)(1 / n-1 / r)} v(t) \in C\left(\left[0, T_{*}\right), L_{r}\left(\mathbb{R}^{n}\right)\right)$ and

$$
\lim _{t \rightarrow+0} t^{(n / 2)(1 / n-1 / r)}\|v(t)\|_{L_{r, \sigma}\left(\mathbb{R}^{n}\right)}=0 .
$$

If , in addition, $v(0) \in L_{n, \sigma}\left(\mathbb{R}^{n}\right) \cap L_{2, \sigma}\left(\mathbb{R}^{n}\right)$ satisfies (9.2.12), then $v$ is also a weak solution of the Navier-Stokes equations on $\left(0, T_{*}\right)$.

Under condition (9.2.12) we can construct a strong solution $v$ on the interval $\left(0, T_{*}\right)$ by the method of successive approximations. To verify (9.2.12) we use the following $L_{p}-L_{r}$ estimates for the Stokes semigroup $\left\{e^{-t S}\right\}_{t \geq 0}$ :

$$
\begin{aligned}
\left\|e^{-t S} a\right\|_{L_{r}\left(\mathbb{R}^{n}\right)} \leq C t^{-(n / 2)(1 / p-1 / r)}\|a\|_{L_{p}\left(\mathbb{R}^{n}\right)}, & 1 \leq p \leq r \leq \infty, \\
\left\|\nabla e^{-t S} a\right\|_{L_{r}\left(\mathbb{R}^{n}\right)} \leq C t^{-(n / 2)(1 / p-1 / r)}\|a\|_{L_{p}\left(\mathbb{R}^{n}\right)}, & 1 \leq p \leq r<\infty,
\end{aligned}
$$

which hold for all $a \in L_{p, \sigma}\left(\mathbb{R}^{n}\right)$ and all $t>0$, where $C=C(n, p, r)$. Hence, if $v(0) \in$ $L_{n, \sigma}\left(\mathbb{R}^{n}\right) \cap L_{r, \sigma}\left(\mathbb{R}^{n}\right)$ for some $n<r<\infty$, then (9.2.12) can be achieved in such a way that

$$
T_{*}=\left(\frac{\gamma}{C\|v(0)\|_{L_{r, \sigma}\left(\mathbb{R}^{n}\right)}}\right)^{2 r /(r-n)}
$$

with the same constant $C$ as in (9.2.14).

Abandoning the smallness assumption we have the open problem of regularity and uniqueness of weak solutions (see Definitions 9.2.1 and 9.2.3). To solve it we can distinguish the following directions:

1. conditional regularity

2. singular and regular points

3. blow-up problems

4. continuation of local strong solutions

5. existence of global regular special solutions

6. decay of solutions.

9.3. Conditional regularity. The classical result on uniqueness and regularity of weak solutions in the class $L_{s}\left(0, T ; L_{r}\left(\mathbb{R}^{n}\right)\right)$ was given by Foiaş [foi], Serrin [ser 1-2], Masuda [mas 1]:

Theorem 9.3.1 (Foiaş-Serrin-Masuda). Let $v(0) \in L_{2, \sigma}\left(\mathbb{R}^{n}\right)$.

(i) Let $u$ and $v$ be two weak solutions of the Navier-Stokes equations on $(0, T)$. Suppose that $u$ satisfies

$$
u \in L_{s}\left(0, T ; L_{r}\left(\mathbb{R}^{n}\right)\right) \quad \text { for } 2 / s+n / r=1 \text { with } n<r \leq \infty .
$$

Assume that $v$ fulfills the energy inequality (9.2.2) for $t \in[0, T)$. Then $u \equiv v$ on $[0, T)$. 
(ii) Every weak solution of the Navier-Stokes equations in the class (9.3.1) satisfies

$$
u \in C^{2,1}\left(\mathbb{R}^{n} \times(0, T)\right) .
$$

The class (9.3.1) is important from the viewpoint of scaling invariance. It is known that if $(v, p)$ is a solution to the Navier-Stokes equations on $\mathbb{R}^{n} \times(0, \infty)$, then so is $v_{\lambda}(x, t)=\lambda v\left(\lambda x, \lambda^{2} t\right), p_{\lambda}(x, t)=\lambda^{2} p\left(\lambda x, \lambda^{2} t\right), \lambda>0$ (see [ckn]). Scaling invariance means that

$$
\left\|v_{\lambda}\right\|_{L_{s}\left(0, T ; L_{r}\left(\mathbb{R}^{n}\right)\right)}=\lambda^{1-(2 / s+n / r)}\|v\|_{L_{s}\left(0, T ; L_{r}\left(\mathbb{R}^{n}\right)\right)}=\|v\|_{L_{s}\left(0, T ; L_{r}\left(\mathbb{R}^{n}\right)\right)}
$$

if and only if $2 / s+n / r=1$.

We shall next deal with the critical case $s=\infty$ and $r=n$ in (9.3.1).

Theorem 9.3.2 (Masuda [mas 1], Kozono-Sohr [kos 1-2]).

(i) (uniqueness) Let $u$ and $v$ be weak solutions to the Navier-Stokes equations. Suppose that $u \in L_{\infty}\left(0, T ; L_{n}\left(\mathbb{R}^{n}\right)\right)$ and $v$ satisfies the energy inequality (9.2.2) for $0 \leq t<T$. Then $u \equiv v$ on $[0, T)$.

(ii) (regularity) There exists a positive constant $\varepsilon_{0}$ such that if $u$ is a weak solution of the Navier-Stokes equations in $L_{\infty}\left(0, T ; L_{n}\left(\mathbb{R}^{n}\right)\right)$ with the property

$$
\lim _{t \rightarrow t_{*}-0} \sup \|u(t)\|_{L_{n}\left(\mathbb{R}^{n}\right)}^{n} \leq\left\|u\left(t_{*}\right)\right\|_{L_{n}\left(\mathbb{R}^{n}\right)}^{n}+\varepsilon_{0} \quad \text { for some } t_{*} \in(0, T),
$$

then

$$
u \in C^{2,1}\left(\mathbb{R}^{n} \times\left(t_{*}-\varrho, t_{*}+\varrho\right)\right) \quad \text { for some } \varrho>0 .
$$

In particular, if $u$ has the property (9.3.3) for every $t_{*} \in(0, T)$, then $u$ is regular on $\mathbb{R}^{n} \times(0, T)$ as in $(9.3 .2)$.

REMARK 9.3.3.

(1) Masuda [mas 1] proved that if $u \in L_{\infty}\left(0, T ; L_{n}\left(\mathbb{R}^{n}\right)\right)$ is continuous from the right on $[0, T)$ in the norm $L_{n}\left(\mathbb{R}^{n}\right)$, then $u \equiv v$ on $[0, T)$. Later on Kozono-Sohr $[\operatorname{kos} 1]$ showed that every weak solution $u$ in $L_{\infty}\left(0, T ; L_{n}\left(\mathbb{R}^{n}\right)\right)$ of the Navier-Stokes equations on $(0, T)$ becomes necessarily continuous from the right in the norm of $L_{n}\left(\mathbb{R}^{n}\right)$.

(2) By the above theorem, every weak solution in $C\left([0, T) ; L_{n}\left(\mathbb{R}^{n}\right)\right)$ is unique and regular. This has been proved by Giga [gig] and von Wahl [wah].

(3) Recently, Hishida-Izumida [hii] have improved the condition (9.3.3). They have proved regularity of $u$ under the weaker assumption

$$
\lim _{t \rightarrow t_{*}-0}\|u(t)\|_{L_{n}\left(\mathbb{R}^{n}\right)}^{n} \leq\left\|u\left(t_{*}\right)\right\|_{L_{n}\left(\mathbb{R}^{n}\right)}^{n}+\varepsilon_{0} .
$$

Theorems 9.3.1, 9.3.2 and Remark 9.3.3 can be found in [koz 1].

Now we recall further results concerning conditional regularity and uniqueness of weak solutions to the Navier-Stokes equations. The first result in this direction was shown by Serrin [ser 1]: 
THEOREM 9.3.4 (Serrin). Let $v$ be a weak solution of the Navier-Stokes equations in some open region $R \subset \mathbb{R}^{n} \times(0, T)$. Suppose that $v \in L_{s, r}(R)$ with

$$
\frac{2}{s}+\frac{n}{r}<1
$$

Then $v$ is of class $C^{\infty}$ in the space variables, and each derivative is bounded in compact subregions of $R$. Assume that $v_{, t} \in L_{2, p}(R)$ with $p \geq 1$. Then the space derivatives of $v$ are absolutely continuous functions of time and $p$ is a strongly differentiable function.

Definition 9.3.5 (Caffarelli-Kohn-Nirenberg [ckn]). A pair $(v, p)$ of measurable functions on $\Omega^{T}$ is called a suitable weak solution of problem $(1.1)_{1,2,5},(1.3)$ with $f=0$ if

1. $v \in L_{\infty}\left(0, T ; L_{2, \sigma}(\Omega)\right) \cap L_{2}\left(0, T ; \stackrel{\circ}{W}_{2, \sigma}^{1}(\Omega)\right), p \in L_{5 / 4}\left(\Omega^{T}\right)$,

2. $v$ is a weak solution of problem $(1.1)_{1,2,5},(1.3)$ and $p$ is an associated pressure,

3. $(v, p)$ satisfies the so called generalized energy inequality

$$
\int_{0}^{T} \int_{\Omega}|\nabla v|^{2} \varphi d x d t \leq \int_{0}^{T} \int_{\Omega}\left[|v|^{2}(\varphi, t+\Delta \varphi)+\left(|v|^{2}+2 p\right) v \cdot \nabla \varphi\right] d x d t
$$

for every infinitely differentiable positive function $\varphi$ on $\Omega^{T}$ with compact support.

Caffarelli-Kohn-Nirenberg [ckn] proved the existence of a suitable weak solution of problem $(1.1)_{1,2,5},(1.3)$ under the assumption that $v(0) \in L_{2, \sigma}(\Omega) \cap W_{5 / 4}^{2 / 5}(\Omega)$.

Definition 9.3.6. A point $(x, t) \in \Omega^{T}$ is called a regular point of the weak solution $v$ if there exists a neighbourhood $U$ of $(x, t) \in \Omega^{T}$ such that $v \in L_{\infty}(U)$. Points of $\Omega^{T}$ which are not regular are called singular. Let us denote by $S(v)$ the set of all singular points of $v$. Then $S(v)$ is closed in $\Omega^{T}$.

A further important result of [ckn] states that if $(v, p)$ is a suitable weak solution of problem $(1.1)_{1,2,5},(1.3)$ then the one-dimensional Hausdorff measure of $S(v)$ equals zero.

Next we recall anisotropic conditional regularity results.

TheOrem 9.3.7 (Neustupa-Penel [nep 1]). Let $(v, p)$ be a suitable weak solution to problem $(1.1)_{1,2,5},(1.3)$ with $f=0$. Suppose that there exists a subdomain $D$ of $\Omega^{T}$ such that $v_{3} \in L_{\infty}(D)\left(v_{3}\right.$ is the third cartesian component of $\left.v\right)$. Then $v$ has no singular points in $D$ (i.e. $S(v) \cap D=\emptyset)$.

Theorem 9.3.8 (Neustupa-Novotny-Penel [nnp]). Let $(v, p)\left(\right.$ where $\left.v=\left(v_{1}, v_{2}, v_{3}\right)\right)$ be a suitable weak solution to problem $(1.1)_{1,2,5},(1.3)$ with $f=0$. Suppose that there exists a subdomain $D \subset \Omega^{T}$ such that $v_{3} \in L_{r, s}(D)$ (where $L_{r, s}\left(\Omega^{T}\right)=L_{r}\left(0, T ; L_{s}(\Omega)\right)$ ) with $r \in[4, \infty], s \in(6, \infty], 2 / r+3 / s \leq 1 / 2$. Then $v$ has no singular points in $D$.

TheOrem 9.3.9 (Neustupa-Penel [nep 2]). Suppose that $(v, p)$ is a suitable weak solution of problem $(1.1)_{1,2,5},(1.3), D$ is an open subdomain in $\Omega^{T}$ and $v=\left(v_{1}, v_{2}, v_{3}\right)$ in Cartesian coordinates. Suppose further that $v_{1}, v_{2} \in L_{p, q, l o c}(D)$ and $v_{3} \in L_{r, s, \text { loc }}(D)$, where $L_{r, s}\left(\Omega^{T}\right)=L_{r}\left(0, T ; L_{s}(\Omega)\right)$ and

1. $p, q \in[2, \infty], r \in[2, \infty), s \in[3, \infty]$,

2. $2 / r+3 / s \leq 1,(2 / p+3 / q)+(2 / r+3 / s) \leq 2,2 / p+2 / r \leq 1,2 / q+2 / s<1$.

Then $(v, p)$ has no singular points in $D$. 
TheOREm 9.3.10 (Neustupa-Penel [nep 2]). Suppose that $D$ is an open subdomain of $\Omega^{T},(v, p)$ is a suitable weak solution of problem $(1.1)_{1,2,5},(1.3), \zeta_{1} \leq \zeta_{2} \leq \zeta_{3}$ are the eigenvalues of the tensor $\left(\frac{1}{2}\left(v_{i, x_{j}}+v_{j, x_{i}}\right)\right)$ and $\zeta_{2}=\zeta_{2}^{1}+\zeta_{2}^{2}$, where

1. $\zeta_{2}^{1} \in L_{\infty, a, \operatorname{loc}}(D)$ for $a>3 / 2$,

2. $\left(\zeta_{2}^{2}\right)_{+} \in L_{1, \infty, \text { loc }}(D)$, where $\left(\zeta_{2}^{2}\right)_{+}$denotes the positive part of $\zeta_{2}^{2}$.

Then $(v, p)$ is regular in $D$.

In [bdv 6] Beirão da Veiga proved regularity of weak solutions to problem $(1.1)_{1,2,5}$ by imposing some conditions on the vorticity $w=\operatorname{rot} v$.

Theorem 9.3.11. Let $v(0) \in L_{2}\left(\mathbb{R}^{n}\right)$, div $v(0)=0, D v(0) \in L_{\alpha^{\prime}}(\Omega)$. Let $v$ be a LerayHopf solution of problem $(1.1)_{1,2,5}$. If $D v \in L_{\alpha}\left(0, T ; L_{\beta}\left(\mathbb{R}^{n}\right)\right.$ with $2 / \alpha+n / \beta=2,1<$ $\alpha \leq \min \{2, n /(n-2)\}$, then

$$
D v \in C\left(0, T ; L_{\alpha^{\prime}}\left(\mathbb{R}^{n}\right)\right) \cap L_{\alpha^{\prime}}\left(0, T ; L_{n \alpha^{\prime} /(n-2)}\left(\mathbb{R}^{n}\right)\right), \quad D v=\left\{v_{i, x_{j}}+v_{j, x_{i}}\right\}_{i, j=1,2,3} .
$$

In the above assumption $D v$ can be replaced by $\operatorname{rot} v$.

Further, we have

Theorem 9.3.12 (Chae-Choe $\left[\right.$ chc]). Let $\Omega=\mathbb{R}^{3}, v(0) \in H_{0, \sigma}^{0}\left(\mathbb{R}^{3}\right), w(0)=\operatorname{rot} v(0) \in$ $H_{0, \sigma}^{0}\left(\mathbb{R}^{3}\right), w=\left(w_{1}, w_{2}, w_{3}\right), w_{1}, w_{2} \in L_{p, q}\left(\Omega^{T}\right), p \in(1, \infty), q \in(3 / 2, \infty), 2 / p+3 / q \leq 2$ or if the norms of $w_{1}$ and $w_{2}$ in $L_{\infty, 3 / 2}\left(\Omega^{T}\right)=L_{\infty}\left(0, T ; L_{3 / 2}(\Omega)\right)$ are sufficiently small. Then $v$ is a classical solution on $(0, T)$ of problem $(1.1)_{1,2,5}$.

Moreover, Chae--Cho [chc] have proved

Theorem 9.3.13. Let $\Omega=\mathbb{R}^{3}, v(0) \in H_{0, \sigma}^{1}\left(\mathbb{R}^{3}\right), \nabla v_{1}, \nabla v_{2} \in L_{p, q}\left(\Omega^{T}\right)=L_{p}\left(0, T ; L_{q}(\Omega)\right)$ with $p \in[2, \infty], q \in[3, \infty], 2 / p+3 / q \leq 1$. Then $v$ is a classical solution on $(0, T)$ of problem $(1.1)_{1,2,5}$.

Finally, Constantin-Fefferman [cof] have proved regularity of a weak solution $v$ under certain assumptions about the behaviour of the quantity rot $v / \operatorname{rot}|v|$.

Now we formulate conditional results where pressure is involved. Put $\varepsilon(t)=\sqrt{t_{0}-t}$ for $t \leq t_{0}$. Assume that $\varrho>0$ and $r>0$. Set

$$
\begin{aligned}
& U_{r}^{\varrho}=\left\{(x, t) \in \mathbb{R}^{3} \times(0, T): t_{0}-r^{2} / \varrho^{2}<t<t_{0}, \varepsilon(t) \varrho<\left|x-x_{0}\right|<r\right\}, \\
& V_{r}^{\varrho}=\left\{(x, t) \in \mathbb{R}^{3} \times(0, T): t_{0}-r^{2} / \varrho^{2}<t<t_{0},\left|x-x_{0}\right|<\varepsilon(t) \varrho\right\} .
\end{aligned}
$$

Let us introduce the conditions

$\left(A_{1}\right)_{r}^{\varrho}: \quad v \in L_{a, b}\left(U_{r}^{\varrho}\right)$ for $a \geq 3, b>3$ such that $2 / a+3 / b=1$,

$\left(A_{2}\right)_{r}^{\varrho}: \quad\|v\|_{L_{\infty, 3}\left(U_{r}^{\varrho}\right)} \leq \varepsilon_{1}$,

$\left(B_{1}\right)_{r}^{\varrho}: \quad p_{-} \in L_{\alpha, \beta}\left(V_{r}^{\varrho}\right)$ for $\alpha \geq 3 / 2, \beta>3 / 2$ such that $2 / \alpha+3 / \beta=2$,

$\left(B_{2}\right)_{r}^{\varrho}: \quad\left\|p_{-}\right\|_{L_{\infty, 3 / 2}\left(V_{r}^{\varrho}\right)}<\varepsilon_{2}$,

where $p_{-}$denotes the negative part of the pressure $p$ : $p_{-}=0$ if $p \geq 0, p_{-}=-p$ if $p<0$. TheOREM 9.3.14 (Nečas-Neustupa [nen]). Suppose that there exist $\varrho>0$ and $r>0$ such that 
(a) condition $\left(A_{1}\right)_{\frac{\varrho}{r}}$ or condition $\left(A_{2}\right)_{r}^{\varrho}$ with sufficiently small $\varepsilon_{1}$ is satisfied, and

(b) condition $\left(B_{1}\right)_{r}^{\varrho}$ or condition $\left(B_{2}\right)_{r}^{\varrho}$ with sufficiently small $\varepsilon_{2}$ is satisfied.

Then $\left(x_{0}, t_{0}\right)$ is a regular point of a suitable weak solution $(v, p)$ of the Cauchy problem $(1.1)_{1,2,5}$.

Let us introduce

Definition 9.3.15. We say that a function $g: \mathbb{R}^{3} \times(0, \infty) \rightarrow[0, \infty)$ satisfies condition (c) if, for any $t_{0}>0$, there exists a positive number $R_{0}=R_{0}\left(t_{0}\right)$ such that

$$
A\left(t_{0}\right) \equiv \sup _{x_{0} \in \mathbb{R}^{3}} \sup _{-R_{0}^{2} \leq t \leq t_{0}} \int_{B\left(x_{0}, R_{0}\right)} \frac{g(x, t)}{\left|x-x_{0}\right|} d x<\infty
$$

and, for each fixed $x_{0} \in \mathbb{R}^{3}$ and each $R \in\left(0, R_{0}\right]$, the function

$$
t \mapsto \int_{B\left(x_{0}, R\right)} \frac{g(x, t)}{\left|x-x_{0}\right|} d x
$$

is continuous at $t_{0}$ from the left.

Seregin-Šverák [ses 1] have proved

ThEOREM 9.3.16. Let $v$ be a Leray-Hopf solution of the Cauchy problem $(1.1)_{1,2,5}$ and let $p$ be the normalized pressure associated with $v$. Assume that there exists a function $g$ satisfying condition (c) such that

$$
|v(x, t)|^{2}+2 p(x, t) \leq g(x, t), \quad x \in \mathbb{R}^{3}, t \in(0, \infty),
$$

or

$$
p(x, t) \geq-g(x, t), \quad x \in \mathbb{R}^{3}, t \in(0, \infty) .
$$

Then $v$ is Hölder continuous on $\mathbb{R}^{3} \times(0, \infty)$ and therefore smooth and unique.

REMARK 9.3.17. Conditions (9.3.8) and (9.3.9) are satisfied if $g=$ const $>0$ in $\mathbb{R}^{3} \times$ $(0, \infty)$.

Finally, we describe conditional regularity results obtained by Beirão da Veiga by methods connected with the regularity techniques used for parabolic equations by De Giorgi-Moser-Ladyzhenskaya and truncation methods (see [lsu]).

Theorem 9.3.18 (Beirão da Veiga [bdv 7]). Let $(v, p)$ be a solution to problem $(1.1)_{1,2,5}$, (1.3). Let $v(0) \in H_{0, \sigma}^{\alpha}(\Omega), \alpha>n$, and $f$ be regular. Let

$$
\frac{p}{1+|v|} \in L_{r}\left(0, T ; L_{q}(\Omega)\right) \quad \text { with } \frac{2}{r}+\frac{n}{q}=1, q \in(n, \infty] .
$$

Then $\left.v \in C(0, T) ; H_{0, \sigma}^{\alpha}(\Omega)\right),|v|^{\alpha / 2} \in L_{2}\left(0, T ; H_{0}^{1}(\Omega)\right)$.

Theorem 9.3.19 (Beirão da Veiga [bdv 1]). Let $(v, p)$ be a solution to problem $(1.1)_{1,2,5}$, (1.3). Let $\|v(0)\|_{L_{\infty}(\Omega)} \leq k_{0}$ for some positive constant $k_{0}$. Let

$$
\varphi_{k}(x, t)= \begin{cases}\frac{|p(x, t)|}{1+|v(x, t)|} & \text { if } v(x, t)>k \\ 0 & \text { otherwise }\end{cases}
$$


and let $\varphi_{k} \in L_{r}\left(0, T ; L_{q}(\Omega)\right)$ with $2 / r+n / q<1, r \in(2, \infty], q \in(n, \infty]$. Then $v \in$ $L_{\infty}\left(\Omega^{T}\right)$. If $p /(1+|v|) \in L_{r}\left(0, T ; L_{q}(\Omega)\right)$ with the same restrictions on $r$ and $q$ as above then $v \in L_{\infty}\left(\Omega^{T}\right)$.

To formulate results from [bdv 2] we need some notation. Let $E$ be a measurable subset of $\mathbb{R}^{n}$ and let $|E|$ be its Lebesgue measure. Let us define the classical Marcinkiewicz space $L_{q, *}(E)$. A measurable function $f(y)$ belongs to $L_{q, *}(E)$ if there exists a constant $[f]_{q}$ such that

$$
|\{x \in E:|f(x)|>\sigma\}| \leq\left([f]_{q} / \sigma\right)^{q}, \quad \forall \sigma>0 .
$$

The smallest constant $[f]_{q}$ for which (9.3.11) holds is called the "norm" of $f$ in $L_{q, *}(E)$. The following algebraic and topological imbeddings hold:

$$
L_{q}(E) \subset L_{q, *}(E) \subset L_{q-\varepsilon}(E), \quad \forall \varepsilon>0 .
$$

Theorem 9.3.20 (Beirão da Veiga [bdv 2]). Let $(v, p)$ be a weak solution of problem $(1.1)_{1,2,5},(1.3)$. Assume that for some $\theta \in[0,1)$ and some $\gamma$ such that

$$
\frac{2(n+2)}{2 \theta+(1-\theta)(n+2)}<\gamma<n+2
$$

one has

$$
\frac{p}{(1+|v|)^{\theta}} \in L_{\gamma, *}\left(\Omega^{T}\right)
$$

Then

$$
v \in L_{\mu, *}\left(\Omega^{T}\right) \quad \text { with } \mu=(1-\theta) \frac{(n+2) \gamma}{n+2-\gamma} .
$$

Moreover, if

$$
\frac{p}{1+|v|} \in L_{\gamma, *}\left(\Omega^{T}\right), \quad \gamma>n+2,
$$

then $v \in L_{\infty}\left(\Omega^{T}\right)$.

To recall results from [bdv 4] we have to introduce some notation. Let us consider problem $(1.1)_{1,2,5},(1.3)$. By a weak solution to problem $(1.1)_{1,2,5}$, (1.3) we mean $v \in$ $C_{w}\left(0, T ; L_{2, \sigma}(\Omega)\right) \cap L_{2}\left(0, T ; H_{0, \sigma}^{1}(\Omega)\right)\left(C_{w}(0, T ; X)\right.$ stands for the continuous functions with respect to the weak topology in $X$ ) satisfying the identity

$$
\begin{aligned}
& \int_{0}^{T}[(v(t), \varphi(t))+\nu(\nabla v(t), \nabla \varphi(t))+(v(t) \cdot \nabla v(t), \varphi(t)) \\
& +(f(t), \varphi(t))] d t=(v(T), \varphi(T))-(v(0), \varphi(0)),
\end{aligned}
$$

for all $\varphi \in C^{1}\left([0, T] ; H_{0, \sigma}^{1}(\Omega)\right)$.

We say that $v$ is a strong solution of the Navier-Stokes equations if

$$
v \in L_{2}(0, T ; D(S)) \cap C\left([0, T] ; H_{0, \sigma}^{1}(\Omega)\right), \quad v_{, t} \in L_{2}\left(0, T ; L_{2, \sigma}(\Omega)\right)
$$

and

$$
\begin{aligned}
& v_{, t}+\nu S v+P(v \cdot \nabla v)=P f \quad \text { in } L_{2}\left(0, T ; L_{2, \sigma}(\Omega)\right), \\
& \left.v\right|_{t=0}=v(0),
\end{aligned}
$$

where $S=-P \Delta$ and $D(S)=H_{0, \sigma}^{1}(\Omega) \cap H^{2}(\Omega)$. 
Let us consider the condition

$$
v \in L_{s}\left(0, T ; L_{r}(\Omega)\right), \quad \frac{2}{s}+\frac{n}{r}=1 \quad \text { and } \quad r>n .
$$

If $n=3$, any weak solution satisfying (9.3.20) is necessarily strong.

Let us introduce the set

$$
A(t, k)=\{x \in \Omega:|v(t, x)|>k\}, \quad k \geq 0, t \in[0, T] .
$$

Hypothesis $A$. We say that $v$ satisfies hypothesis $A$ at $\bar{t}$ (with constant $C$ ) if $v \in$ $L_{\infty}\left(0, T ; L_{n}(\Omega)\right)$ and if there are $\delta>0$ and a real nonnegative function $k(t)$ defined and square integrable on $(\bar{t}-\delta, \bar{t})$ such that

$$
\int_{A(t, k(t))}|v(t, x)|^{n} d x \leq C^{n} \quad \text { a.e. in }(\bar{t}-\delta, \bar{t}) .
$$

We say that $v$ satisfies hypothesis $A$ in $[0, T]$ if it satisfies hypothesis $A$ at each $\bar{t} \in(0, T]$; here $\delta$ and $k(t)$ may depend on the particular point $\bar{t}$.

TheOREM 9.3.21 (Beirão da Veiga [bdv 4]). Let $v$ be a weak solution of problem $(1.1)_{1,2,5}$, (1.3). Assume that for some $\bar{t} \in(0, T], v$ is a strong solution in $[0, \tau]$ for each $\tau<\bar{t}$ and moreover $v$ satisfies hypothesis $A$ at $\bar{t}$ with constant $C_{0}$. Then $v \in C\left(0, \bar{t} ; H_{0, \sigma}^{1}(\Omega)\right)$.

Finally, we have

Theorem 9.3.22 (Beirão da Veiga [bdv 3]). Suppose $v(0) \in L_{2, \sigma}(\Omega)$ and $D v(0) \in L_{p}(\Omega)$ for some $p \geq \max \{2, n / 2\}$. Suppose $v$ is a Leray-Hopf solution of problem $(1.1)_{1,2,5}$. If

$$
D v \in L_{p^{\prime}}\left(0, T ; L_{p n / 2}\left(\mathbb{R}^{n}\right)\right), \quad \frac{1}{p}+\frac{1}{p^{\prime}}=1,
$$

then

$$
D v \in C\left(0, T ; L_{p}\left(\mathbb{R}^{n}\right)\right) \cap L_{p}\left(0, T ; L_{p n /(n-2)}\left(\mathbb{R}^{n}\right)\right) .
$$

Moreover,

$$
\begin{aligned}
& \sup _{0 \leq t \leq T}\|D(v(t))\|_{L_{p}\left(\mathbb{R}^{n}\right)}^{p}+\int_{0}^{T}\|D v(t)\|_{L_{p n /(n-2)}}^{p}\left(\mathbb{R}^{n}\right) \\
& \\
& \quad \leq c\|D v(0)\|_{L_{p}\left(\mathbb{R}^{n}\right)}^{p}\left[1+\exp \left(c \int_{0}^{T}\|D v(t)\|_{L_{n p / 2}\left(\mathbb{R}^{n}\right)}^{p^{\prime}} d t\right)\right] .
\end{aligned}
$$

Finally, we recall results concerning axially symmetric solutions and their properties. Global existence of axially symmetric solutions was considered by Leonardi-MálekNečas-Pokorný [lmnp 1-2] and by Gallagher-Ibrahim-Majdoub [gima]. Properties of axially symmetric solutions were examined by Neustupa-Pokorny [npo 1-2, pok]. Recently Seregin-Šverák [ses 2] have proved that $v \in L_{\infty}\left(0, T ; L_{3}(\Omega)\right)$ implies regularity of a weak solution.

9.4. Singular and regular points. Caffarelli-Kohn-Nirenberg [ckn] have shown that $\mathcal{H}^{1}(S)=0$, where $\mathcal{H}^{1}(S)$ is the one-dimensional Hausdorff measure and $S$ is the set of singular points introduced in Definition 9.3.6. Recently Choe-Lewis [chs] have obtained 
a better estimate of the Hausdorff dimension of $S$. Neustupa [neu] has described the set of singular points.

TheOREM 9.4.1 (Neustupa [neu]). Let $n=3$. Then there is an absolute constant $\varepsilon_{0}>0$ such that every weak solution $v$ in $L_{\infty}\left(0, T ; L_{3}(\Omega)\right)$ fulfills

$$
\# S(v) \leq\left(\frac{1}{\varepsilon_{0}} \sup _{0<t<T}\|v(t)\|_{L_{3}(\Omega)}\right)^{3}
$$

where $\# S$ denotes the number of elements of the set $S$.

Recently Kozono [koz 2] has found deeper characteristics of the singular set.

Theorem 9.4.2 (Kozono [koz 2], see also [koz 1]). Let $n=3$. There is an absolute constant $\varepsilon_{0}>0$ with the following property. If $v$ is a weak solution of the Navier-Stokes equations on $(0, T)$ and if $v$ satisfies

$$
\sup _{t_{0}-\varrho<t<t_{0}+\varrho}\|v(t)\|_{L_{3, W}\left(B\left(x_{0}, \delta\right)\right)} \leq \varepsilon_{0}
$$

at $\left(x_{0}, t_{0}\right) \in \mathbb{R}^{3} \times(0, T)$ for some $\delta>0, \varrho>0$, then $\left(x_{0}, t_{0}\right)$ is a regular point.

Here $\|\cdot\|_{L_{3, W}\left(B\left(x_{0}, \delta\right)\right)}=\sup _{R>0} R \mu\left\{x \in B\left(x_{0}, \delta\right):|v(x)|>R\right\}^{1 / 2}$, where $\mu$ is the Lebesgue measure.

Corollary 9.4.3 (removable singularities, Kozono [koz 1]). Let $n=3$. There is an absolute constant $\varepsilon_{0}$ such that if $v$ is a weak solution to the Navier-Stokes equations on $(0, T)$ and $\left(x_{0}, t_{0}\right)$ is an isolated singular point of $v$ satisfying

$$
\lim _{x \rightarrow x_{0}} \sup _{t \rightarrow t_{0}}\left|x-x_{0}\right| v(x, t) \mid<\varepsilon_{0},
$$

then $\left(x_{0}, t_{0}\right)$ is a regular point.

In particular, if $v$ behaves at $\left(x_{0}, t_{0}\right)$ like

$$
v(x, t)=o\left(\left|x-x_{0}\right|^{-1}\right) \quad \text { as } x \rightarrow x_{0}
$$

uniformly with respect to $t$ in some neighbourhood of $t_{0}$, then $\left(x_{0}, t_{0}\right)$ is a regular point.

Remark 9.4.4. Kozono [koz 1], Serrin [ser 1] and Takahashi [tak] have shown that every weak solution $v$ of the Navier-Stokes equations satisfying

$$
\|v\|_{L_{s}\left(a, b ; L_{r}(D)\right)}<\infty \quad \text { for } D \times(a, b) \subset \Omega \times(0, T), \frac{2}{s}+\frac{3}{r} \leq 1, \quad r>3,
$$

is of class $C^{\infty}$ in the space variables. Theorem 9.4.2 deals with the marginal case $s=$ $\infty, r=3$ and $L_{3}(D) \subset L_{3, w}(D)$. Moreover, Theorem 9.4.2 implies interior regularity in the space-time variables but Serrin [ser 1] had to impose additionally that $v_{, t} \in$ $L_{s}\left(a, b ; L_{2}(D)\right)$ with $s \geq 1$.

Caffarelli-Kohn-Nirenberg [ckn] have found an absolute constant $\varepsilon_{1}$ such that if the suitable weak solution satisfies

$$
R^{-2} \int_{Q_{R}\left(x_{0}, t_{0}\right)}\left(|v|^{3}+|v||p|\right) d x d t+R^{-13 / 4} \int_{t_{0}-R^{2}}^{t_{0}}\left(\int_{\left|x-x_{0}\right|<R}|p| d x\right)^{5 / 4} d t \leq \varepsilon_{1},
$$


where $Q_{R}\left(x_{0}, t_{0}\right)=\left\{(x, t):\left|x-x_{0}\right|<R, t_{0}-R^{2}<t<t_{0}\right\}$, then $v$ is regular in $Q_{R / 2}\left(x_{0}, t_{0}\right)$.

Theorem 9.4.2 generalizes this result because Corollary 9.4.3 implies that

$$
\int_{Q_{R}\left(x_{0}, t_{0}\right)}|v(x, t)|^{3} d x d t=\infty
$$

which is excluded by (9.4.4).

9.5. Blow-up problems. Leray [ler] has proposed a construction of singular solutions to the Cauchy problem $(1.1)_{1,2,5}$ by looking for $v$ and $p$ in the form

$$
v(x, t)=\frac{1}{\sqrt{2 a\left(t_{0}-t\right)}} V\left(\frac{x-x_{0}}{\sqrt{2 a\left(t_{0}-t\right)}}\right), \quad p(x, t)=\frac{1}{2 a\left(t_{0}-t\right)} P\left(\frac{x-x_{0}}{\sqrt{2 a\left(t_{0}-t\right)}}\right)
$$

where $a>0$ and $V, P$ are solutions of the problem

$$
\begin{array}{ll}
V \cdot \nabla V=-\nabla P+\nu \Delta V-a V-a x \cdot \nabla V & \text { in } \mathbb{R}^{3}, \\
\operatorname{div} V=0 & \text { in } \mathbb{R}^{3} .
\end{array}
$$

The existence of nontrivial solutions of problem (9.5.2) would easily lead to the singularity of $v, p$ at the point $\left(x_{0}, t_{0}\right)$. However, the following result holds:

Theorem 9.5.1 (Nečas-Růžička-Šverák [nrs]). If $V, P$ is a solution of (9.5.2), then $V \equiv 0$.

9.6. Continuation of the local strong solution. Let $v(0) \in H_{\sigma}^{s}\left(\mathbb{R}^{n}\right)$ with $s>n / 2-1$. Then Fujita-Kato [fuk 1] have shown that there exists $T=T\left(\|v(0)\|_{H_{\sigma}^{s}\left(\mathbb{R}^{n}\right)}\right)>0$ and a unique solution $v(t)$ to the Cauchy problem $(1.1)_{1,2,5}$ such that

$$
v \in C\left([0, T) ; H_{\sigma}^{s}\left(\mathbb{R}^{n}\right)\right) \cap C^{1}\left((0, T) ; H^{s}\left(\mathbb{R}^{n}\right)\right) \cap C\left((0, T] ; H^{s+2}\left(\mathbb{R}^{n}\right)\right) .
$$

Since $s>n / 2-1$, we have the imbedding $H^{s}\left(\mathbb{R}^{n}\right) \subset L_{r}\left(\mathbb{R}^{n}\right), r>n$. Hence the time $T\left(\|v(0)\|_{H_{\sigma}^{s}\left(\mathbb{R}^{n}\right)}\right)$ is characterized by $(9.2 .15)$.

The following problem appears: either the solution $v(t)$ loses its regularity at $t=T$ or there exists $T^{\prime}>T$ such that (9.6.1) holds with $T^{\prime}$ in place of $T$.

Giga [gig] and Beale-Kato-Majda [bkm] have given a criterion for extension of strong solutions.

TheOREM 9.6.1. Let $v(0) \in H_{\sigma}^{s}\left(\mathbb{R}^{n}\right), s>n / 2-1$. Suppose that $v$ is a solution of the Navier-Stokes equations on $[0, T)$ in the class (9.6.1). If either

$$
\int_{0}^{T}\|v(t)\|_{L_{r}\left(\mathbb{R}^{n}\right)}^{k} d t<\infty \quad \text { for } 2 / k+n / r=1, n<r<\infty,
$$

or

$$
\int_{0}^{T}\|\operatorname{rot} v(t)\|_{L_{\infty}\left(\mathbb{R}^{n}\right)} d t<\infty,
$$

then there exists $T^{\prime}>T$ such that $v(t)$ can be continued to a solution on $\left[0, T^{\prime}\right)$ in the class (9.6.1). 
Using [zaj 6] Theorem 9.6.1 can be extended to solutions for the Cauchy-Dirichlet problem $(1.1)_{1,2,5},(1.3)$.

Let us define the BMO space by the norm

$$
\|f\|_{\mathrm{BMO}}=\sup _{B} \frac{1}{|B|} \int_{B}\left|f(x)-f_{B}\right| d x<\infty,
$$

where $B$ denotes a ball in $\mathbb{R}^{n}$ and $f_{B}=|B|^{-1} \int_{B} f(x) d x$. In the above definition, the supremum is taken with respect to all balls $B$ in $\mathbb{R}^{n}$.

Theorem 9.6.2 (Kozono-Taniuchi [kta], Kozono [koz 1]). Let $s>n / 2-1$ and let $v(0) \in$ $H_{\sigma}^{s}\left(\mathbb{R}^{n}\right)$. Suppose that $v$ is a strong solution of the Navier-Stokes equations in the class (9.6.1). Assume that $v$ satisfies either

$$
\int_{\varepsilon_{0}}^{T}\|v(t)\|_{\mathrm{BMO}}^{2} d t<\infty \quad \text { or } \quad \int_{\varepsilon_{0}}^{T}\|\operatorname{rot} v(t)\|_{\mathrm{BMO}} d t<\infty
$$

for some $\varepsilon_{0} \in(0, T)$. Then $v$ can be continued to a solution on $\left[0, T^{\prime}\right)$ in the class (9.6.1) for some $T^{\prime}>T$.

TheOrem 9.6.3 (Kozono [koz 1]). Let $v$ be a solution of the Navier-Stokes equations in the class (9.6.1) for $s>n / 2-1$. Suppose that $T$ is maximal, i.e. $v$ cannot be continued in the class (9.6.1) for any $T^{\prime}>T$. Then

$$
\int_{\varepsilon}^{T}\|v(t)\|_{\mathrm{BMO}}^{2} d t=\int_{\varepsilon}^{T}\|\operatorname{rot} v(t)\|_{\mathrm{BMO}} d t=\infty
$$

for all $\varepsilon \in(0, T)$. In particular,

$$
\limsup _{t \nearrow T}\|v(t)\|_{\mathrm{BMO}}=\limsup _{t \nearrow T}\|\operatorname{rot} v(t)\|_{\mathrm{BMO}}=\infty .
$$

In the above theorem \|\|$_{\text {BMO }}$ can be replaced by \|\|$_{\dot{B}_{\infty, \infty}^{0}}$, where $\dot{B}_{p, q}^{s}$ is the homogeneous Besov space (see Kozono-Ogawa-Taniuchi [kot]).

9.7. Global special regular solutions. The aim of this section is to present results on global regular solutions to the Navier-Stokes equations which are proved under some geometrical and analytical restrictions. The results are proved under some symmetry assumptions. Up to now three kinds of global regular solutions to the Navier-Stokes equations are known:

1. two-dimensional solutions - Ladyzhenskaya [lad 2];

2. axially symmetric solutions, in all space - Yudovich-Ukhovskij [uky] and in a cylinder with the axis of symmetry removed — Ladyzhenskaya [lad 1];

3. helically symmetric solutions - Mahalov-Leibovich-Titi [mlt].

The above solutions, in fact two-dimensional, have been generalized to three dimensions by examining their stability by Ponce-Racke-Sideris-Titi [prst] and by StröhmerZajączkowski [stz].

A generalization of the two-dimensional solutions from [lad 2] was done by Sell, Raugel, Iftimie and Avrin. In [ras] Raugel and Sell have proved existence of global regular 
solutions in a thin domain $\Omega_{\varepsilon}=\Omega^{\prime} \times(0, \varepsilon), \Omega^{\prime} \subset \mathbb{R}^{2}, \varepsilon$ small and with periodic boundary conditions by using the semigroup technique.

Theorem 9.7.1 (Raugel-Sell [ras 1]). Consider problem $(1.1)_{1,2,5}$ on $\Omega_{\varepsilon}$ with periodic boundary conditions. There is an $\varepsilon_{0}=\varepsilon_{0}\left(\nu, \lambda_{1}\right)>0$, where $\lambda_{1}=4 \pi^{2} l_{1}^{-2}$ is the smallest eigenvalue of $-P \Delta$ on $\Omega_{\varepsilon}=\left(0, l_{1}\right) \times\left(0, l_{2}\right) \times(0, \varepsilon), l_{1} \geq l_{2}>\varepsilon$, such that for every $\varepsilon \in\left(0, \varepsilon_{0}\right]$ there are large sets $\mathcal{R}(\varepsilon)$ and $\mathcal{S}(\varepsilon)$,

$$
\begin{aligned}
& \mathcal{R}(\varepsilon) \subset\left\{u \in H^{1}\left(\Omega_{\varepsilon}\right): \operatorname{div} u=0, \int_{\Omega_{\varepsilon}} u d x=0\right\}, \\
& \mathcal{S}(\varepsilon) \subset\left\{f \in W_{\infty}^{1}\left([0, \infty) ; L_{2}\left(\Omega_{\varepsilon}\right)\right): \int_{\Omega_{\varepsilon}} f d x=0\right\},
\end{aligned}
$$

such that if $v(0) \in \mathcal{R}(\varepsilon)$ and $f \in \mathcal{S}(\varepsilon)$, then $(1.1)_{1,2,5}$ has a strong solution $v(t)$ with $\left.v\right|_{t=0}=v(0)$, defined for all $t \geq 0$ and

$$
\|v(t)\|_{H^{1}\left(\Omega_{\varepsilon}\right)} \leq k_{1}<\infty,
$$

where $k_{1}$ depends on $v(0)$ and $f$. Furthermore, there exist constants $l_{1}$ and $l_{2}$ which do not depend on $v(0)$ and which satisfy

$$
\limsup _{t \rightarrow \infty}\|v(t)\|_{H^{1}\left(\Omega_{\varepsilon}\right)} \leq l_{1}, \quad \limsup _{t \rightarrow \infty}\|v(t)\|_{H^{2}\left(\Omega_{\varepsilon}\right)} \leq l_{2} .
$$

This result was generalized by Avrin [avr 1-2] who also proved existence of global regular solutions in the thin domain $\Omega_{\varepsilon}=\Omega^{\prime} \times(0, \varepsilon), \Omega^{\prime} \subset \mathbb{R}^{2}, \varepsilon$ small, with Dirichlet boundary conditions on $\partial \Omega^{\prime}$ and periodic conditions in the third direction. In his considerations the smallness of $\varepsilon$ was replaced by large first eigenvalue of $-P \Delta$, where $P$ is the projection operator on the divergence free vector fields. To prove existence he used a fixed point argument. A generalization of the above results was given by Iftimie-Raugel [ifr] who relaxed the conditions on the magnitude and regularity of $v(0)$ and $f$.

Constantin-Fefferman [cof] have proved that uniformly Lipschitz (for all times) estimate of variation of direction of the vorticity vector implies regularity of solutions to the Navier-Stokes equations. A generalization of the result has been given by BabinMahalov-Nicolaenko (see [bmn 1-2]). They have proved existence of global regular solutions to the Navier-Stokes equations for initial data characterized by uniformly large vorticity. They consider the following problem:

$$
\begin{aligned}
& v_{, t}+v \cdot \nabla v-\nu \Delta v+\nabla p=0, \\
& \operatorname{div} v=0,
\end{aligned}
$$

$$
\left.v\right|_{t=0}=v_{0}+\frac{1}{2} \Omega \bar{e}_{3} \times \bar{x}
$$

where $\bar{e}_{3}=(0,0,1), \bar{x}=\left(x_{1}, x_{2}, x_{3}\right), \Omega$ is a constant.

TheOREM 9.7.2 ([bmn 1]). Let positive numbers $a_{1}, a_{2}, a_{3}$ be given. Let the initial condition $v_{0}$ be defined on the x-periodic lattice $T_{a_{1} a_{2} a_{3}}=\left[0,2 \pi a_{1}\right] \times\left[0,2 \pi a_{2}\right] \times\left[0,2 \pi a_{3}\right]$ with zero mean. Let $v_{0} \in L_{2}\left(T_{a_{1} a_{2} a_{3}}\right)$ and $\left\|v_{0}\right\|_{L_{2}\left(T_{a_{1} a_{2} a_{3}}\right)} \leq M_{0}$. Then there exist $T_{*}=$ $T_{*}\left(M_{0}, a_{1}, a_{2}, a_{3}, \nu\right)$ and $\Omega_{*}=\Omega_{*}\left(a_{1}, a_{2}, a_{3}, \nu\right)$ such that for every $\Omega \geq \Omega_{*}, v(x, t)$ can be extended to $t \in(0, \infty)$ and it is regular for $t \in\left(T_{*}, \infty\right)$. Moreover $v(x, t) \in H_{r}^{1}\left(\mathbb{R}^{3}\right)$, 
$r>5 / 2, \operatorname{rot} v \in L_{2, \mathrm{loc}}\left(\mathbb{R}^{3}\right)$ for $t>T_{*}$ and

$$
\|v(t)\|_{H_{r}^{1}\left(\mathbb{R}^{3}\right)} \leq C_{1}\left(a_{1}, a_{2}, a_{3}, \nu\right) .
$$

In the above formulation $H_{r}^{s}\left(\mathbb{R}^{3}\right)$ is the weighted space with the norm

$$
\|u\|_{H_{r}^{s}\left(\mathbb{R}^{3}\right)}=\left(\sum_{|\alpha| \leq s} \int_{\mathbb{R}^{3}} \frac{1}{\left(1+|x|^{2}\right)^{r}}\left|D_{x}^{\alpha} u\right|^{2} d x\right)^{1 / 2} .
$$

In this paper we prove global existence of solutions to problem (1.1) which are close to axially symmetric solutions.

DeFinition 9.7.3. By an axially symmetric solution to problem (1.1) we mean a solution such that $v_{\varphi}=0, f_{\varphi}=0, v(0)_{\varphi}=0, v_{r, \varphi}=0, v_{z, \varphi}=0, f_{r, \varphi}=0, f_{z, \varphi}=0, p_{, \varphi}=0$.

The main result of this paper can be expected as a classical stability result for the axially symmetric solution. Let $v=v_{a}(r, z, t), p=p_{a}(r, z, t)$ be the axially symmetric solution to problem (1.1) with the r.h.s. $f=f_{a}(r, z, t)$. Then the disturbances $v^{\prime}=v-v_{a}$, $p^{\prime}=p-p_{a}$ are solutions to the problem

$$
\begin{aligned}
& v_{, t}^{\prime}+v^{\prime} \cdot \nabla v^{\prime}+v_{a} \cdot \nabla v^{\prime}+v^{\prime} \cdot \nabla v_{a}-\nu \Delta v^{\prime}+\nabla p^{\prime}=f^{\prime}, \\
& \operatorname{div} v^{\prime}=0, \\
& \left.v^{\prime} \cdot \bar{n}\right|_{S}=0, \\
& \left.\left(\bar{n} \cdot \mathbb{T}\left(v^{\prime}, p^{\prime}\right) \cdot \bar{\tau}_{\alpha}+\gamma v^{\prime} \cdot \bar{\tau}_{\alpha}\right)\right|_{S}=0, \quad \alpha=1,2, \\
& \left.v^{\prime}\right|_{t=0}=v^{\prime}(0) .
\end{aligned}
$$

Having a sufficiently regular axially symmetric solution we are able to prove the existence of global regular solutions to (9.7.2) for small data.

However we do not know how to prove the existence of global axially symmetric solutions in the rectangle $0<r<R,-a<z<a$ in spaces with the weight equal to a power function of $r$.

Appearance of this kind of weighted spaces is connected with the global energy type estimate for the $\chi$-component of vorticity (see Section 4). Therefore we consider problem (1.1) directly in three dimensions because in this case we are able to treat elliptic and parabolic problems in weighted spaces.

Moreover, we underline that the result of this paper is not a stability result in the above sense. We shall show this by contradiction. Assume that we are looking for a stability result. Then employing the quantities $h$ and $q$ we have

$$
\begin{aligned}
v^{\prime} & =v-v_{a}=\int_{\varphi_{0}}^{\varphi} h_{r} d \varphi \bar{e}_{r}+\int_{\varphi_{0}}^{\varphi} h_{\varphi} d \varphi \bar{e}_{\varphi}+\int_{\varphi_{0}}^{\varphi} h_{z} d \varphi \bar{e}_{z} \equiv H \\
p^{\prime}=p-p_{a} & =\int_{\varphi_{0}}^{\varphi} q d \varphi
\end{aligned}
$$

where $\varphi_{0}$ is any number from $[0,2 \pi]$. Since $v_{a}$ satisfies $(9.7 .2)_{2}$ we have to check that so 
does $H$ modulo that $h$ is a solution to (1.4). Since

$$
H_{r}=\int_{\varphi_{0}}^{\varphi} h_{r} d \varphi, \quad H_{\varphi}=\int_{\varphi_{0}}^{\varphi} h_{\varphi} d \varphi, \quad H_{z}=\int_{\varphi_{0}}^{\varphi} h_{z} d \varphi,
$$

we calculate

$$
\begin{aligned}
\operatorname{div} H= & H_{r, r}+\frac{1}{r} H_{\varphi, \varphi}+H_{z, z}+\frac{H_{r}}{r}=\int_{\varphi_{0}}^{\varphi} h_{r, r} d \varphi+\frac{1}{r}\left(\int_{\varphi_{0}}^{\varphi} h_{\varphi} d \varphi\right)_{, \varphi} \\
& +\int_{\varphi_{0}}^{\varphi} h_{z, z} d \varphi+\frac{1}{r} \int_{\varphi_{0}}^{\varphi} h d \varphi=\int_{\varphi_{0}}^{\varphi}\left(h_{r, r}+h_{z, z}+\frac{h_{r}}{r}\right)+\frac{1}{r} h_{\varphi} \\
= & -\int_{\varphi_{0}}^{\varphi} \frac{1}{r} h_{\varphi, \varphi} d \varphi+\frac{1}{r} h_{\varphi}=\frac{1}{r} h_{\varphi}\left(\varphi_{0}\right) \neq 0 .
\end{aligned}
$$

Similarly, $H$ does not satisfy $(9.7 .2)_{1}$.

9.8. Decay of solutions. Time asymptotics and stability of solutions to the NavierStokes equations in $\mathbb{R}^{n}$ and in exterior domains were examined intensively by Schonbek [sch 1-2], Schonbek-Wiegner [scw], Wiegner [wie 1-5], Kajikiya-Miyakawa [kam], and Galdi-Maremonti [gam]. 


\section{References}

[ama] H. Amann, On the strong solvability of the Navier-Stokes equations, J. Math. Fluid Mech. 2 (2000), 16-98.

[avr 1] J. D. Avrin, Large-eigenvalue global existence and regularity results for the NavierStokes equations, J. Differential Equations 127 (1996), 365-390.

[avr 2] - - A one-point attractor theory for the Navier-Stokes equations on thin domains with no-slip boundary conditions, Proc. Amer. Math. Soc. 127 (1999), 725-735.

[bmn 1] A. Babin, A. Mahalov and B. Nicolaenko, 3D Navier-Stokes and Euler equations with initial data characterized by uniformly large vorticity, Indiana Univ. Math. J. 50 (2001), 1-35.

[bmn 2] - - - - Global regularity of $3 D$ rotating Navier-Stokes equations for resonant domains, ibid. 48 (1999), 1133-1176.

[bkm] J. T. Beale, T. Kato and A. Majda, Remarks on the breakdown of smooth solutions for the 3D Euler equations, Comm. Math. Phys. 94 (1984), 61-66.

[bdv 1] H. Beirão da Veiga, Concerning the regularity of the solutions to the Navier-Stokes equations via the truncation method. I, Differential Integral Equations 10 (1997), $1149-1156$.

[bdv 2] - Concerning the regularity of the solutions to the Navier-Stokes equations via the truncation method. II, in: Équations aux derivées partielles et applications, Elsevier, Paris, 1998, 127-138.

[bdv 3] -, A new regularity class for the Navier-Stokes equations in $\mathbb{R}^{n}$, Chinese Ann. Math. Ser. B 16 (1995), 407-412.

[bdv 4] - Remarks on the smoothness of the $L^{\infty}\left(0, T ; L^{3}\right)$ solutions of the 3-D Navier-Stokes equations, Portugal. Math. 54 (1997), 381-391.

[bdv 5] - Existence and asymptotic behavior for strong solutions of the Navier-Stokes equations in the whole space, Indiana Univ. Math. J. 36 (1987), 149-166.

[bdv 6] - Concerning the regularity problem for the solutions of the Navier-Stokes equations, C. R. Acad. Sci. Paris Sér I 321 (1995), 405-408.

[bdv 7] - A sufficient condition on the pressure for the regularity of weak solutions to the Navier-Stokes equations, J. Math. Fluid. Mech. 2 (2000), 99-106.

[bin] O. V. Besov, V. P. Il'in and S. M. Nikol'skiǔ, Integral Representations of Functions and Imbedding Theorems, Nauka, Moscow, 1975 (in Russian).

[bre] H. Brezis, Remarks on the preceding paper by M. Ben-Artzi, "Global solutions of two-dimensional Navier-Stokes and Euler equations", Arch. Rat. Mech. Anal. 128 (1994), 359-360.

[ckn] L. Caffarelli, R. Kohn and L. Nirenberg, Partial regularity of suitable weak solutions of the Navier-Stokes equations, Comm. Pure Appl. Math. 35 (1982), 771-831.

[cal 1] C. P. Calderón, Existence of weak solutions for the Navier-Stokes equations with initial data in $L_{p}$, Trans. Amer. Math. Soc. 318 (1990), 179-200.

[cal 2] - Addendum to the paper "Existence of weak solutions for the Navier-Stokes equations with initial data in $L_{p}$ ", ibid. 318 (1990), 201-207. 
[cal 3] C. P. Calderón, Initial values of solutions of the Navier-Stokes equations, Proc. Amer. Math. Soc. 117 (1993), 761-766.

[can] M. Cannone, Ondelettes, Paraproduits et Navier-Stokes, Diderot, Paris, 1995.

[cam] M. Cannone and Y. Meyer, Littlewood-Paley decomposition and Navier-Stokes equations, Methods Appl. Anal. 2 (1995), 307-319.

[chc] D. Chae and H. J. Choe, Regularity of solutions to the Navier-Stokes equations, Electronic J. Differential Equations 5 (1999), 1-7.

[chs] H. J. Choe and J. L. Lewis, On the singular set in the Navier-Stokes equations, J. Funct. Anal. 175 (2000), 348-369.

[cof] P. Constantin and C. Fefferman, Direction of vorticity and the problem of global regularity for the Navier-Stokes equations, Indiana Univ. Math. J. 42 (1993), 775-789.

[fjr] E. B. Fabes, B. F. Jones and N. M. Rivière, The initial value problem for the NavierStokes equations with data in $L_{p}$, Arch. Rat. Mech. Anal. 45 (1972), 222-240.

[flr] E. B. Fabes, J. E. Lewis and N. M. Rivière, Boundary value problems for the NavierStokes equations, Amer. J. Math. 99 (1977), 626-668.

[foi] C. Foiaş, Une remarque sur l'unicité des solutions des équations de Navier-Stokes en dimension n, Bull. Soc. Math. France 89 (1961), 1-8.

[fuk 1] H. Fujita and T. Kato, On the Navier-Stokes initial value problem I, Arch. Rat. Mech. Anal. 16 (1964), 269-315.

[fuk 2] - - - On the nonstationary Navier-Stokes system, Rend. Sem. Mat. Univ. Padova 32 (1962), 243-260.

[gal] G. P. Galdi, An Introduction to the Navier-Stokes Initial-Boundary Value Problem, Vols. I-II, Springer, 1994.

[gam] G. P. Galdi and P. Maremonti, Monotonic decreasing and asymptotic behaviour of the kinetic energy for weak solutions of the Navier-Stokes equations in exterior domains, Arch. Rat. Mech. Anal. 94 (1986), 253-266.

[gima] I. Gallagher, S. Ibrahim and M. Majdoub, Existence et unicité de solutions pour le système de Navier-Stokes axisymmétrique, Comm. Partial Differential Equations 26 (2001), 883-907.

[gig] Y. Giga, Solutions for semilinear parabolic equations in $L^{p}$ and regularity of weak solutions of the Navier-Stokes system, J. Differential Equations 62 (1986), 186-212.

[gim] Y. Giga and T. Miyakawa, Solution in $L_{r}$ of the Navier-Stokes initial value problem, Arch. Rat. Mech. Anal. 89 (1985), 267-281.

[hii] T. Hishida and K. Izumida, Remarks on a regularity criterion for weak solutions to the Navier-Stokes equations in $\mathbb{R}^{n}$, Analysis 20 (2000), 191-200.

[hop] E. Hopf, Über die Anfangwertaufgabe für die hydrodynamischen Grundgleichungen, Math. Nachr. 4 (1951), 213-231.

[ifr] D. Iftimie and G. Raugel, Some results on the Navier-Stokes equations in thin 3D domains, J. Differential Equations 169 (2001), 281-331.

[iln] V. P. Il'in, On inequalities between norms of partial derivatives of functions with several variables, Trudy Mat. Inst. Steklov. 84 (1965), 144-173 (in Russian).

[kam] R. Kajikiya and T. Miyakawa, On $L^{2}$ decay of weak solutions of the Navier-Stokes equations in $\mathbb{R}^{n}$, Math. Z. 192 (1986), 135-148.

[kat 1] T. Kato, Strong $L_{p}$-solutions of the Navier-Stokes in $\mathbb{R}^{n}$, with applications to weak solutions, Math. Z. 187 (1984), 471-480.

[kat 2] -, Quasilinear equations of evolution with applications to partial differential equations, in: Lecture Notes in Math. 448, Springer, 1975, 25-70.

[kap] T. Kato and G. Ponce, Commutator estimates and the Euler and Navier-Stokes equations, Comm. Pure Appl. Math. 41 (1988), 891-907.

[kil] A. Kiselev and O. A. Ladyzhenskaya, On the existence and uniqueness of the solution of the non-stationary problem for a viscous incompressible fluid, Izv. Akad. Nauk SSSR Ser. Mat. 21 (1957), 655-680 (in Russian). 
[koc] N. E. Kochin, Vectorial Calculus and Introduction to Tensorial Calculus, Akad. Nauk SSSR, Moscow, 1951 (in Russian).

[kkr 1] N. E. Kochin, I. A. Kibel' and N. V. Roze, Theoretical Hydrodynamics, Moscow, 1963 (in Russian).

[kom] G. Komatsu, Global analyticity up to the boundary of solutions of the Navier-Stokes equations, Comm. Pure Appl. Math. 33 (1980), 545-566.

[kon] V. A. Kondrat'ev, Boundary value problems for elliptic equations in domains with conical and angular points, Trudy Moskov. Mat. Obshch. 16 (1967), 209-292 (in Russian).

[koz 1] H. Kozono, On well-posedness of the Navier-Stokes equations, in: Mathematical Fluid Mechanics, I. Neustupa and P. Penel (eds.), Birkhäuser, 2001, 207-236.

[koz 2] -, Removable singularities of weak solutions to the Navier-Stokes equations, Comm. Partial Differential Equations 23 (1998), 949-966.

[kot] H. Kozono, T. Ogawa and Y. Taniuchi, The critical Sobolev inequalities in Besov spaces and regularity criterion to some semi-linear evolution equations, Math. Z. 242 (2002), 251-278.

[kos 1] H. Kozono and H. Sohr, Remark on uniqueness of weak solutions to the Navier-Stokes equations, Analysis 16 (1996), 255-271.

[kos 2] - - - Regularity criterion on weak solutions to the Navier-Stokes equations, Adv. Differential Equations 2 (1997), 535-554.

[kta] H. Kozono and Y. Taniuchi, Bilinear estimates in BMO and the Navier-Stokes equations, Math. Z. 235 (2000), 173-194.

[lad 1] O. A. Ladyzhenskaya, On unique solvability of the three-dimensional Cauchy problem for the Navier-Stokes equations under axial symmetry, Zap. Nauchn. Sem. LOMI 7 (1968) 155-177 (in Russian).

[lad 2] - Solutions "in the large" of the nonstationary boundary value problem for the Navier-Stokes system with two space variables, Comm. Pure Appl. Math. 12 (1959), 427-433.

[lad 3] - The Mathematical Theory of Viscous Incompressible Flow, Nauka, Moscow, 1970 (in Russian).

[lsu] O. A. Ladyzhenskaya, V. A. Solonnikov and N. N. Ural'tseva, Linear and Quasilinear Equations of Parabolic Type, Nauka, Moscow, 1967 (in Russian).

[lal] L. Landau and E. Lifshitz, Hydrodynamics, Nauka, Moscow, 1986 (in Russian).

[lmnp 1] S. Leonardi, J. Málek, J. Nečas and M. Pokorný, On axially symmetric flows in $\mathbb{R}^{3}$, Z. Anal. Anwend. 18 (1999), 639-649.

[lmnp 2] - , - - - - On the result of Ukhovskii and Yudovich on axially symmetric flows of a viscous fluid in $\mathbb{R}^{3}$, preprint 522, Bonn, 1997.

[ler] J. Leray, Sur le mouvement d'un liquide visqueux emplissant l'espace, Acta Math. 63 (1934), 193-248.

[lew] J. E. Lewis, The initial-boundary value problem for the Navier-Stokes equations with data in $L^{p}$, Indiana Univ. Math. J. 22 (1973), 739-761.

[mlt] A. Mahalov, E. S. Titi and S. Leibovich, Invariant helical subspaces for the NavierStokes equations, Arch. Rat. Mech. Anal. 112 (1990), 193-222.

[mas 1] K. Masuda, Weak solutions of Navier-Stokes equations, Tohoku Math. J. 36 (1984), 623-646.

[mas 2] - On the analyticity and unique continuation theorem for solutions of the NavierStokes equations, Proc. Japan Acad. 43 (1967), 827-832.

[map] V. G. Maz'ya and B. A. Plamenevskǐ̌, $L_{p}$-estimates for solutions of elliptic boundary value problems in domains with edges, Trudy Moskov. Mat. Obshch. 37 (1978), 49-93 (in Russian). 
[nen] J. Nečas and J. Neustupa, New conditions for local regularity of a suitable weak solution to the Navier-Stokes equation, J. Math. Fluid Mech. 4 (2002), 1-20.

[nrs] J. Nečas, M. Růžička and V. Šverák, On Leray's self-similar solutions of the NavierStokes equations, Acta Math. 176 (1996), 283-294.

[neu] J. Neustupa, Partial regularity of weak solutions to the Navier-Stokes equations in the class $L^{\infty}\left(0, T ; L^{3}(\Omega)^{3}\right)$, J. Math. Fluid Mech. 1 (1999), 309-325.

[nnp] J. Neustupa, A. Novotný and P. Penel, A remark to the interior regularity of a suitable weak solution to the Navier-Stokes equations, to appear.

[nep 1] J. Neustupa and P. Penel, Regularity of a suitable weak solutions to the Navier-Stokes equations as a consequence of regularity of one velocity component, in: Nonlinear Applied Analysis, A. Sequeira, H. Beirão da Veiga and J. Videman (eds.), Plenum Press, New York, 1999, 391-402.

[nep 2] -, 一, Anisotropic and geometric criteria for interior regularity of weak solutions to the $3 d$ Navier-Stokes equations, in: Mathematical Fluid Mechanics, J. Neustupa and P. Penel (eds.). Birkhäuser, 2001, 237-265.

[npo 1] J. Neustupa and M. Pokorný, Axisymmetric flow of Newtonian fluid in the whole space with non-zero tangential velocity component, to appear.

[npo 2] - - - An interior regularity criterion for an axially symmetric suitable weak solution to the Navier-Stokes equations, J. Math. Fluid Mech. 2 (2000), 381-399.

[pok] M. Pokorný, A regularity criterion for the angular velocity component in the case of axisymmetic Navier-Stokes equations, in: Elliptic and Parabolic Problems, World Sci., 2002, 233-242.

[prst] G. Ponce, R. Racke, T. C. Sideris and E. S. Titi, Global stability of large solutions to the 3D Navier-Stokes equations, Comm. Math. Phys. 159 (1994), 329-341.

[ras 1] G. Raugel and G. R. Sell, Navier-Stokes equations on thin 3D domains. I: Global attractors and global regularity of solutions, J. Amer. Math. Soc. 6 (1993), 503-568.

[ras 2] -, 一, Navier-Stokes equations on thin 3D domains. II: Global regularity of spatially periodic solutions, in: Nonlinear Partial Differential Equations and Their Applications, Collège de France Seminar, Vol. XI, H. Brezis and J.-L. Lions (eds.), Pitman Res. Notes Math. Ser. 299, Longman, Essex, 1994, 205-247.

[ras 3] -, 一, Navier-Stokes equations on thin 3D domains. III: Global and local attractors, in: Turbulence in Fluid Flows: A Dynamic Systems Approach, IMA Vol. Math. Appl. 55, G. R. Sell et al. (eds.), Springer, New York, 1993, 137-163.

[rib] F. Ribaud, Semilinear parabolic equations with distributions as initial data, Discrete Cont. Dynam. Systems 3 (1997), 305-316.

[sch 1] M. E. Schonbek, $L^{2}$ decay for weak solutions of the Navier-Stokes equations, Arch. Rat. Mech. Anal. 88 (1985), 209-222.

[sch 2] - Lower bounds of rates of decay for solutions to the Navier-Stokes equations, J. Amer. Math. Soc. 4 (1991), 423-449.

[scw] M. E. Schonbek and M. Wiegner, On the decay of higher-order norms of the solutions of Navier-Stokes equations, Proc. Roy. Soc. Edinburgh Sect. A 126 (1996), 677-685.

[ses 1] G. Seregin and V. Šverák, On solutions to the Navier-Stokes equations with lower bounds on pressure, to appear.

[ses 2] - - - The Navier-Stokes equations and backward uniqueness, in: Nonlinear Problems in Mathematical Physics and Related Topics II, Kluwer, 2002, 353-366.

[ser 1] J. Serrin, On the interior regularity of weak solutions of the Navier-Stokes equations, Arch. Rat. Mech. Anal. 9 (1962), 187-195.

[ser 2] - The initial value problem for the Navier-Stokes equations, in: Nonlinear Problems, R. E. Langer (ed.), Univ. of Wisconsin Press, Madison, 1963, 69-98.

[soh] H. Sohr, The Navier-Stokes Equations, An Elementary Functional Analytic Approach, Birkhäuser, Basel, 2001. 
[sol] V. A. Solonnikov, On the classical solvability of initial-boundary value problems for the heat equation in a dihedral angle, Zap. Nauchn. Sem. LOMI 138 (1984), 146-180 (in Russian).

[sos] V. A. Solonnikov and V. E. Shchadilov, On a boundary value problem for a stationary system of Navier-Stokes equations, Trudy Mat Inst. Steklov. 125 (1973), 196-210 (in Russian); English transl.: Proc. Steklov Inst. Math. 125 (1973), 186-199.

[soz] V. A. Solonnikov and W. M. Zajączkowski, On the Neumann problem for second order elliptic equations in domains with edges on the boundary, Zap. Nauchn. Sem. LOMI 127 (1983), 7-48 (in Russian).

[stz] G. Ströhmer and W. M. Zajączkowski, Existence and stability theorems for abstract parabolic equations, and some of their applications, in: Singularities and Differential Equations, Banach Center Publ. 33, Inst. Math., Polish Acad. Sci., 1996, 369-382.

[tak] S. Takahashi, On interior regularity criteria for weak solutions of the Navier-Stokes equations, Manuscripta Math. 69 (1990), 237-254.

[uky] M. R. Ukhovskiu and V. I. Yudovich, Axially symmetric motions of ideal and viscous fluids filling all space, Prikl. Mat. Mekh. 32 (1968), 59-69 (in Russian).

[wah] W. von Wahl, Regularity of weak solutions of the Navier-Stokes equations, in: Nonlinear Functional Analysis and Its Applications, Proc. Sympos. Pure Math. 45, Part 2, F. E. Browder (ed.), Amer. Math. Soc., Providence, RI, 1986, 497-503.

[wie 1] M. Wiegner, Decay results for weak solutions of the Navier-Stokes equations in $\mathbb{R}^{n}$, J. London Math. Soc. 35 (1987), 303-313.

[wie 2] - Decay and stability in $L_{p}$ for strong solutions to the Cauchy problem for the Navier-Stokes equations, in: Proc. Oberwolfach 1988, Lecture Notes in Math. 1431, Springer, 1990, 95-99.

[wie 3] - Approximation of weak solutions of the Navier-Stokes equations in unbounded domains, in: Proc. Oberwolfach 1991, Lecture Notes in Math. 1530, Springer, 1992, 161-166.

[wie 4] - Decay of the $L_{\infty}$-norm of solutions to the Navier-Stokes equations in unbounded domains, Acta Appl. Math. 37 (1994), 215-219.

[wie 5] —, The Navier-Stokes equations - a neverending challenge?, Jber. Deutsch. Math.Verein. 101 (1999), 1-25.

[zaj 1] W. M. Zajączkowski, Existence of solutions of initial-boundary value problems for the heat equation in Sobolev spaces with the weight as a power of the distance to some axis, in: Proc. 3rd Polish Symposium on Nonlinear Analysis, Lecture Notes in Nonlinear Analysis 3 (2002), 185-192.

[zaj 2] - Existence of solutions to some elliptic system in Sobolev spaces with the weight as a power of the distance to some axis, Topol. Methods Nonlinear Anal. 19 (2002), 91-108.

[zaj 3] - On imbedding theorems for weighted anisotropic Sobolev spaces, Appl. Math. (Warsaw) 29 (2002), 51-63.

[zaj 4] - Existence of solutions vanishing near some axis for the nonstationary Stokes system with boundary slip conditions, Dissert. Math. 400 (2002).

[zaj 5] - Global special regular solutions to the Navier-Stokes equations in a cylindrical domain under boundary slip conditions, Gakuto Series in Math. 21, 2004.

[zaj 6] - Remarks on the breakdown of smooth solutions for the 3-d Euler equations in a bounded domain, Bull. Polish Acad. Sci. Math. 37 (1989), 169-181.

[zaj 7] - Global existence of axially symmetric solutions to Navier-Stokes equations with large angular component of velocity, Colloq. Math. 100 (2004), 243-263.

[zaj 8] - Global special regular solutions to the Navier-Stokes equations in a cylindrical domain without the axis of symmetry, Topol. Methods. Nonlinear Anal. 24 (2004), 69-105. 
[zaj 9] W. M. Zajacczkowski, Long time existence of regular solutions to Navier-Stokes equations in cylindrical domains under boundary slip conditions, Studia Math. 169 (2005), 243-285.

[zaj 10] - Global regular nonstationary flow for the Navier-Stokes equations in a cylindrical pipe, Topol. Methods Nonlinear Anal. (2005).

[zaj 11] - Global axially symmetric solutions with large swirl to the Navier-Stokes equations, to appear.

[zaj 12] -, Global nonstationary Navier-Stokes motions in cylindrical pipes, Gakuto Series in Math. (2005). 